\title{
Quality of Water and Chemistry of Bottom Sediment in the Rillito Creek Basin, Tucson, Arizona, 1986-92 By SAEID TADAYON and CHRISTOPHER F. SMITH
}

U.S. GEOLOGICAL SURVEY

Water-Resources Investigations Report 94-4114

Prepared in cooperation with the ARIZONA DEPARTMENT OF WATER RESOURCES and PIMA COUNTY DEPARTMENT OF TRANSPORTATION AND FLOOD CONTROL DISTRICT

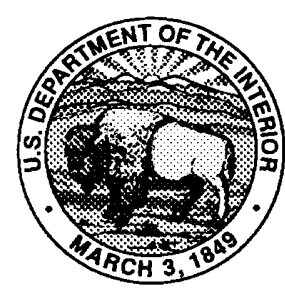




\title{
U.S. DEPARTMENT OF THE INTERIOR BRUCE BABBITT, Secretary
}

\author{
U.S. GEOLOGICAL SURVEY
}

Gordon P. Eaton, Director

Any use of trade, product, or firm names in this publication is for descriptive purposes only and does not constitute endorsement by the U.S. Government.

For additional information write to:

District Chief

U.S. Geological Survey

Water Resources Division

375 South Euclid Avenue

Tucson, AZ 85719-6644
Copies of this report can be purchased from:

U.S. Geological Survey

Earth Science Information Center

Open-File Reports Section

Box 25286, MS 517

Denver Federal Center

Denver, CO 80225 


\section{CONTENTS}

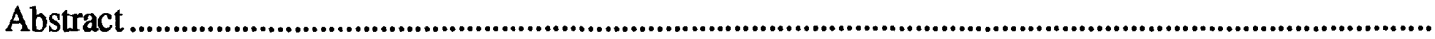

Introduction

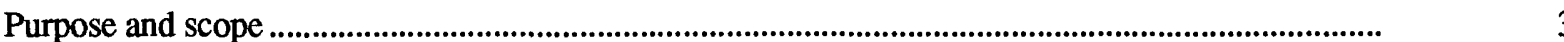

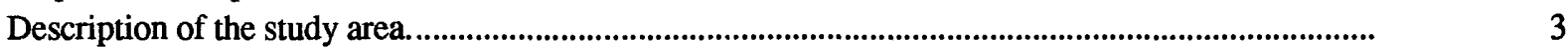

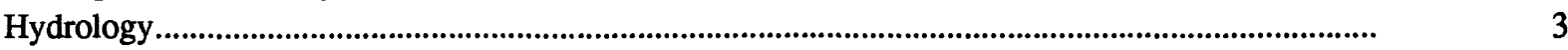

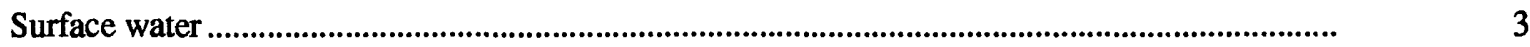

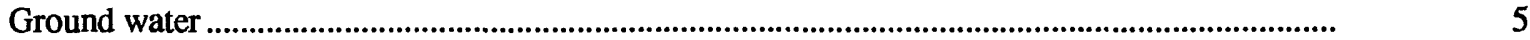

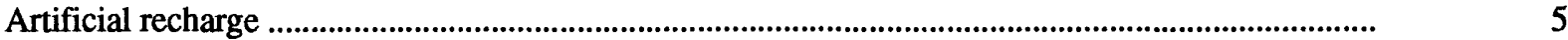

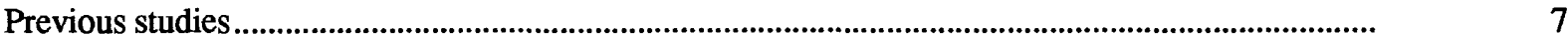

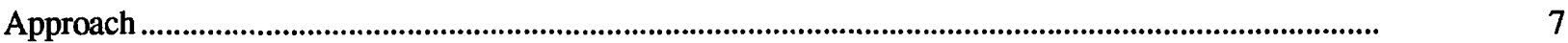

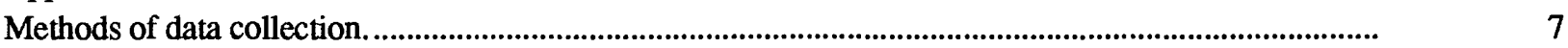

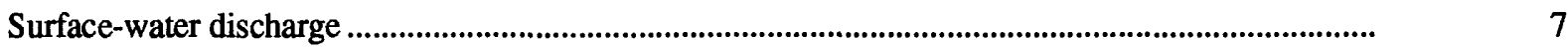

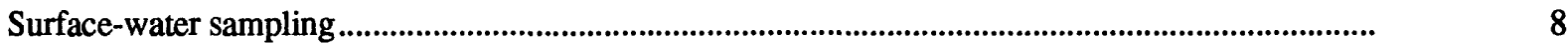

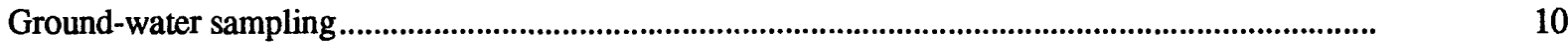

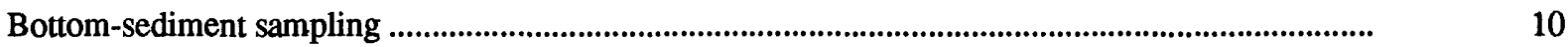

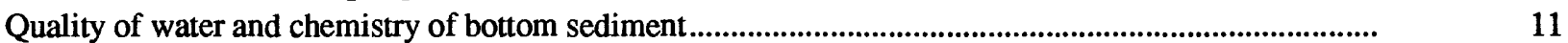

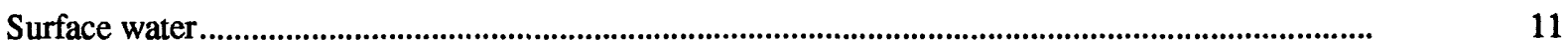

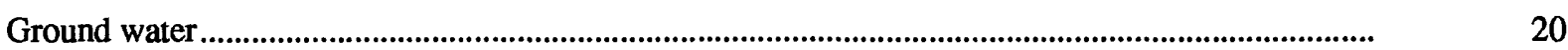

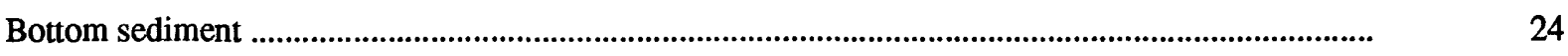

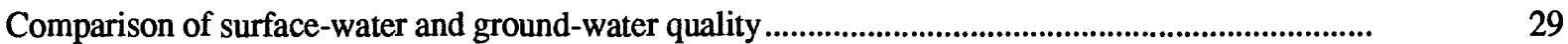

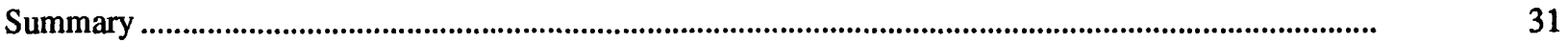

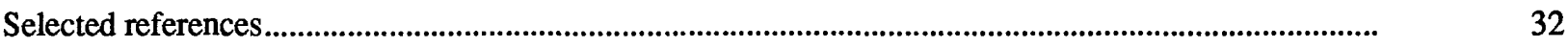

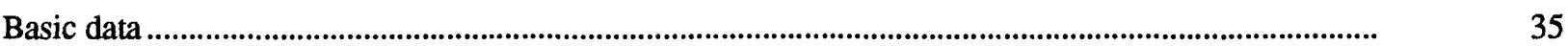

\section{FIGURES}

1. Map showing location of study area and surface-water and ground-water data-collection sites, Rillito Creek basin

2. Map showing location of Rillito Creek in the upper Santa Cruz drainage basin

3. Hydrograph of flow in Tanque Verde Creek and water levels in observation well (D-13-14)26cbb2, 1986-92.

4. Trilinear diagram showing relative compositions of surface water, in percent, Rillito Creek basin, 1987-92

5. Graphs showing relation between selected total recoverable trace-element concentration and suspended-sediment concentration in surface-water samples, Rillito Creek basin...........

6. Trilinear diagram showing relative compositions of ground water, in percent, Rillito Creek basin, 1986-89

7-15. Hydrographs of flow in Tanque Verde Creek and water level in observation wells, 1986-92:

7. Well (D-13-14)26bbb.

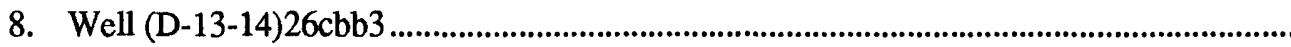

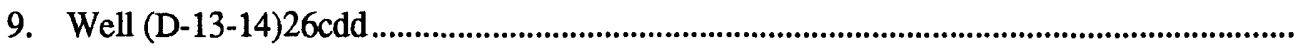

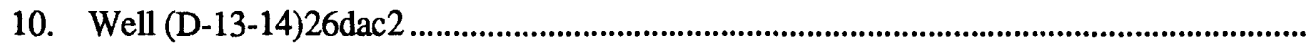

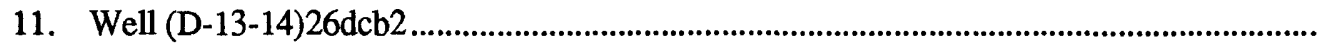

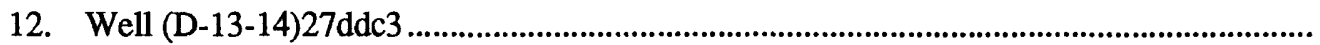

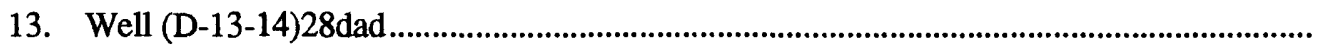

14. Well (D-13-14)34aaal .............................................................................................

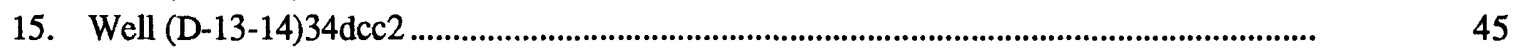


1. Well-construction information for selected wells, Rillito Creek basin ............................................... 11

2. Summary of selected physical and chemical data for surface-water sites, Rillito Creek basin, February 25, 1987, through March 13, 1992 ..............

3. Summary of selected physical and chemical data for ground-water sites, Rillito Creek basin, August 25, 1986, to March 28, 1989

4. Summary of selected physical and chemical data for bottom-sediment sites, Rillito Creek basin, July 28, 1987, through February 20, 1992 ...........

5. Trace-element concentrations in bottom sediment of the Rillito Creek basin and in soils of the western conterminous United States

6. Median values of physical and chemical data for surface-water and ground-water samples, Rillito Creek basin, August 25, 1986, through February 13, 1992 ......

7. Suspended-sediment concentration and particle-size distribution of surface-water samples, Rillito Creek basin

8. Analytical results of surface-water samples, Rillito Creek basin.

9. Analytical results of ground-water samples, Rillito Creek basin

10. Particle-size distribution of bottom-sediment samples, Rillito Creek basin ...................................

11. Analytical results of bottom-sediment samples, Rillito Creek basin

\section{CONVERSION FACTORS}

\begin{tabular}{lll}
\hline \multicolumn{1}{c}{ Multiply inch-pound unit } & By & To obtaln metric unit \\
\hline inch (in.) & 25.4 & millimeter \\
foot $(\mathrm{ft})$ & 0.3048 & meter \\
mile $(\mathrm{mi})$ & 1.609 & kilometer \\
square mile $\left(\mathrm{mi}^{2}\right.$ ) & 2.590 & square kilometer \\
cubic feet per second $\left(\mathrm{ft}^{3} / \mathrm{s}\right)$ & 0.0283 & cubic meter per second \\
gallon per minute $(\mathrm{gal} / \mathrm{min})$ & 0.06309 & liter per second \\
foot per year $(\mathrm{ft} / \mathrm{yr})$ & 0.3048 & meter per year \\
acre-foot (acre-ft) & 0.001233 & cubic hectometer \\
\hline
\end{tabular}

In this report, degrees are reported in Celsius $\left({ }^{\circ} \mathrm{C}\right)$, which can be converted to degrees Fahrenheit $\left({ }^{\circ} \mathrm{F}\right)$ by the following equation:

$$
{ }^{\circ} \mathrm{F}=1.8\left({ }^{\circ} \mathrm{C}\right)+32
$$

\section{ABBREVIATED UNITS FOR WATER QUALITY AND BOTTOM-SEDIMENT CHEMISTRY}

Chemical concentration and water temperature are given only in metric units. Chemical concentration in water is given in milligrams per liter $(\mathrm{mg} / \mathrm{L})$ or micrograms per liter $(\mu \mathrm{g} / \mathrm{L})$. Milligrams per liter is a unit expressing the solute per unit volume (liter) of water. One thousand micrograms per liter is equivalent to 1 milligram per liter. For concentrations less than 7,000 milligrams per liter, the numerical value is about the same as for concentrations in parts per million. Specific conductance is given in microsiemens per centimeter $(\mu \mathrm{S} / \mathrm{cm})$ at $25^{\circ} \mathrm{C}$. Radioactivity is expressed in picocuries per liter $(\mathrm{pCi} / \mathrm{L})$ or picocuries per gram $(\mathrm{pCi} / \mathrm{g})$, which is the amount of radioactive decay producing 2.2 disintegrations per minute in a unit volume (liter) of water or volume (gram) of sediment. Chemical concentration in bottom sediment is given in grams per kilogram $(\mathrm{g} / \mathrm{kg})$, micrograms per gram $(\mu \mathrm{g} / \mathrm{g})$, milligrams per kilogram $(\mathrm{mg} / \mathrm{kg})$, or micrograms per kilogram $(\mu \mathrm{g} / \mathrm{kg})$. Grams per kilogram is equal to parts per thousand (ppt). Milligrams per kilogram and micrograms per gram are equal to parts per million (ppm). Micrograms per kilogram is equal to parts per billion (ppb).

\section{VERTICAL DATUM}

Sea Level: In this report, "sea level" refers to the National Geodetic Vertical Datum of 1929-a geodetic datum derived from a general adjustment of the first-order level nets of the United States and Canada, formerly called Sea Level Datum of 1929. 


\section{WELL-NUMBERING AND NAMING SYSTEM}

R. 14 E.

WELL (D-13-14)26dcb2

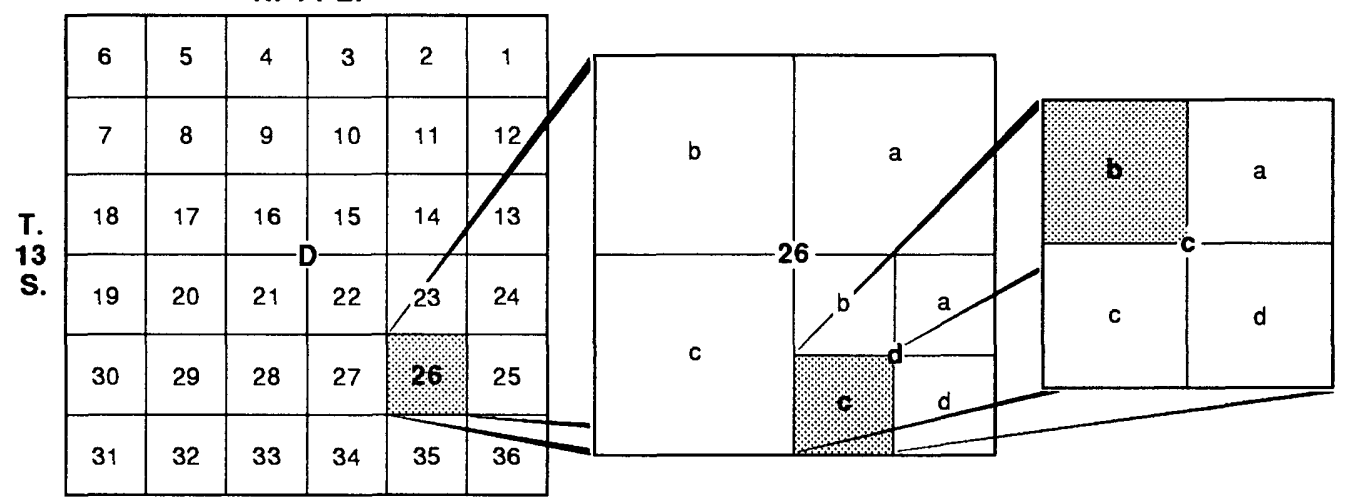

Quadrant D, Township 13 South, Range 14 East, section 26, quarter section d, quarter section $c$, quarter section $b$, second well inventoried in 10 -acre tract

The well numbers used by the U.S. Geological Survey in Arizona are in accordance with the Bureau of Land Management's system of land subdivision. The land survey in Arizona is based on the Gila and Salt River meridian and base line, which divide the state into four quadrants and are designated by capital letters A, B, C, and D in a counterclockwise direction, beginning in the northeast quarter. The first digit of a well number indicates the township, the second the range, and the third the section in which the well is situated. The lowercase letters a, b, c, and $\mathrm{d}$ after the section number indicate the well location within the section. The first letter denotes a particular 160 -acre tract, the second the 40 -acre tract and the third the 10 -acre tract. These letters also are assigned in a counterclockwise direction, beginning in the northeast quarter. If the location is known within the 10-acre tract, three lowercase letters are shown in the well number. Where more than one well is within a 10 -acre tract, consecutive numbers beginning with 1 are added as suffixes. In the example shown, well number (D-13-14)26dcb2 designates the well as being in the NW1/4, SW1/4, SE1/4, section 26, Township 13 South, and Range 14 East. 


\title{
Quality of Water and Chemistry of Bottom Sediment in the Rillito Creek Basin, Tucson, Arizona, 1986-92
}

\author{
By Saeid Tadayon and Christopher F. Smith
}

\section{Abstract}

Controlled artificial recharge of surface runoff is being considered as a water-management technique to address the problem of ground-water overdraft in Rillito Creek basin, Arizona. Surface-water, ground-water, and bottom-sediment data were collected from August 25, 1986, through March 13, 1992, to provide information that would be needed to plan and manage artificial recharge operations.

Suspended-sediment concentrations in streams generally increased with increases in streamflow and were highest during the summer. The surface water is a calcium and bicarbonate type, and the ground water is a calcium, sodium, and bicarbonate type. Total recoverable trace elements in surface water that exceeded the State of Arizona maximum contaminant levels for drinking water were barium, cadmium, chromium, lead, and mercury. None of the dissolved trace elements in surface water exceeded the drinking-water standards. The median values for dissolved activities of gross beta as strontium-90/yttrium- 90 and dissolved gross beta as cesium- 137 were lower in ground water than in surface water. Comparisons of trace-element concentrations in bottom sediment with those reported for soils of the western conterminous United States generally indicate similar concentrations for most of the trace elements, with the exceptions of scandium and tin. The maximum concentrations of total nitrite plus nitrate as nitrogen in three ground-water samples exceeded U.S. Environmental Protection Agency maximum contaminant levels for drinking water. Seven organochlorine pesticides were detected in surface-water samples and ten were detected in bottom-sediment samples. Three priority pollutants were detected in surface water, two were detected in ground water, and eleven were detected in bottom sediment. Low concentrations of oil and grease were detected in 7 of 25 surface-water samples.

\section{INTRODUCTION}

Controlled artificial recharge of surface runoff is being considered as a water-management technique to address the problem of ground-water overdraft. The U.S. Bureau of Reclamation High Plains States Groundwater Demonstration Program suggested the Rillito Creek in Tucson, Arizona, as a site to study the feasibility of using stormwater runoff for artificial recharge. The Pima County Department of Transportation and Flood Control District (PCFCD) in cooperation with the U.S. Bureau of Reclamation is developing plans for the implementation of a proposed ground-water recharge project in a 1-mile reach of the Rillito Creek between Craycroft Road and Swan Road in the north-central part of Tucson (fig. 1). The proposed ground-water recharge project in Rillito Creek will utilize runoff for infiltration and recharge purposes within the channel and excavated overbank areas. This proposed recharge would be accomplished by water spreading and detention using an inflatable dam.

In urban areas, the use of recharge facilities has caused concern about the quality of urban runoff and the potential for ground-water contamination. Runoff from developed areas is exposed to a broad range of contaminant sources, and the presence of particular contaminants may depend on the type of land use. Little is known of the chemical quality of runoff from a southwestern 


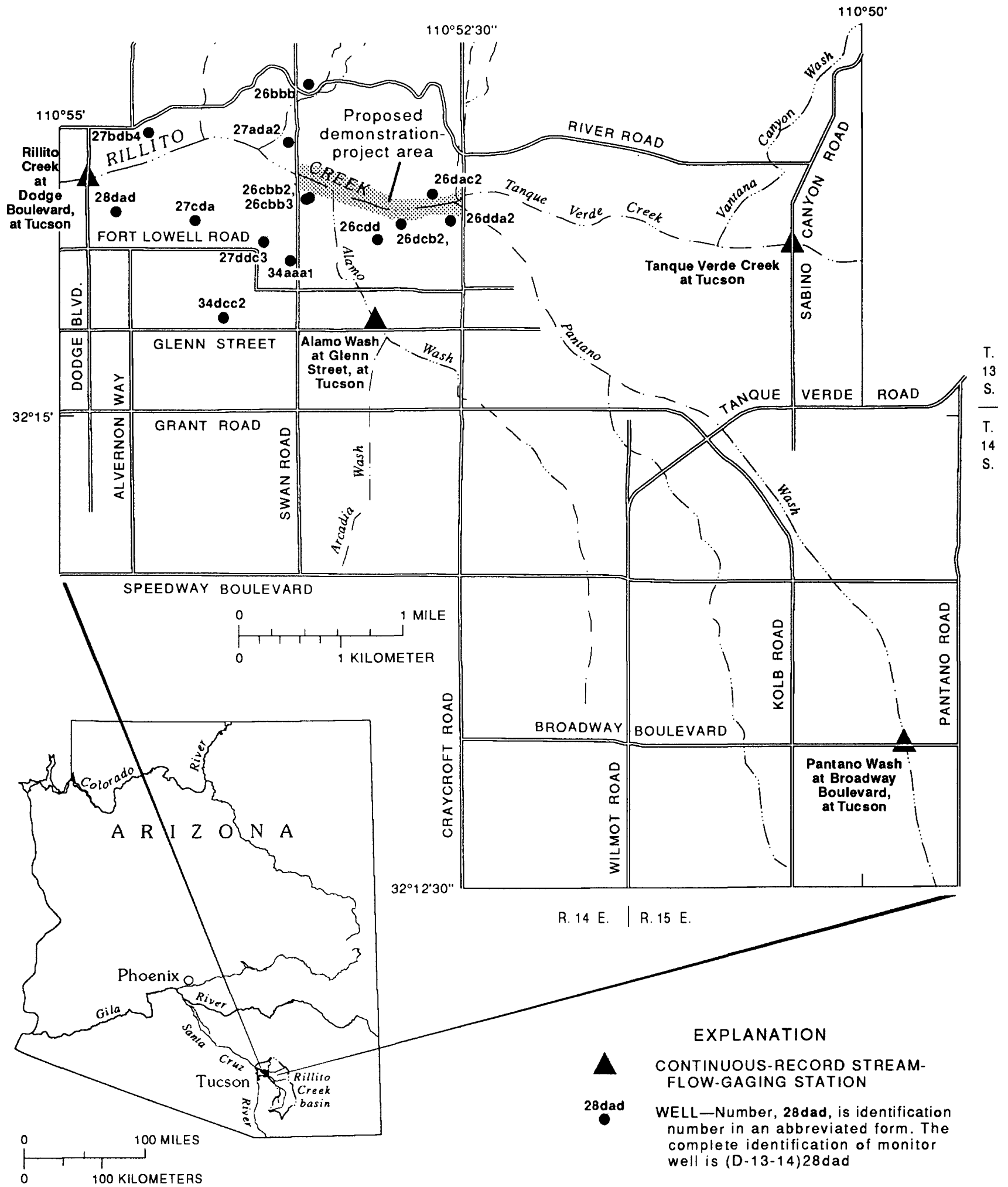

Figure 1. Location of study area and surface-water and ground-water data-collection sites, Rillito Creek basin. 
urbanized environment and even less about the potential for contamination of ground water by recharge of urban runoff in the Tucson area. In 1986, the U.S. Geological Survey (USGS) in cooperation with the Arizona Department of Water Resources (ADWR), and PCFCD began collecting baseline physical and chemical data from surface water, ground water, and bottom sediment in the Rillito Creek basin.

\section{Purpose and Scope}

The purpose of this report is to (1) present physical and chemical data of surface water, ground water, and bottom sediment and (2) compare the quality of surface water with that of ground water in the study area. This study includes the collection and analysis of physical and chemical data from 4 surface-water, 14 ground-water, and 4 bottom-sediment sites. Data for this study were collected from August 25, 1986, through March 13, 1992.

\section{Description of the Study Area}

The Tucson basin is a broad 1,000-squaremile area in the upper Santa Cruz drainage basin in southern Arizona (Laney, 1972). The basin is about $50 \mathrm{mi}$ long and is 15 to $20 \mathrm{mi}$ wide in the southern and central parts and $4 \mathrm{mi}$ wide at the northwest outlet (Davidson, 1973). The basin is bounded on the north by the Tortolita and Santa Catalina Mountains; on the east by the Rincon Mountains; on the south by the Santa Rita Mountains; and on the west by the Sierrita, Black, and Tucson Mountains (fig. 2). These mountains consist of igneous, metamorphic, and sedimentary rocks from Precambrian to late Tertiary age (Davidson, 1973). The basin is underlain by several thousand feet of unconsolidated and semiconsolidated alluvial material (Burkham, 1970). The primary stratigraphic units of the Tucson basin are the Pantano Formation of Oligocene age, the Tinaja beds of Miocene and Pliocene age, and the Fort Lowell Formation of Pleistocene age (Davidson, 1973). The Pantano Formation consists of silty sandstone to gravel that is cemented by calcium carbonate (Davidson, 1973). The Pantano Formation contains a few interbedded volcanic flows and tuffs and are as much as thousands of feet thick (Davidson, 1973; Anderson, 1987). The Tinaja beds unconformably overlie the Pantano Formation and are unconformably overlain by the Fort Lowell Formation. The Tinaja beds consist of clayey silt, mudstone, and gravel and are as much as 5,000 ft thick (Davidson, 1973). The Fort Lowell Formation overlies the Tinaja beds and is overlain by surficial deposits. The Fort Lowell Formation, which consists of silty gravel near the margin of the basin to a silty sand and clayey silt in the central part of the basin, is 300 to $400 \mathrm{ft}$ thick in most of the basin and thins toward the mountains (Davidson, 1973). In some areas of the Tucson basin, the surficial deposits include alluvial-fan, sheetflow, and stream-channel deposits overlying the older sedimentary units and range from a thin veneer to tens of feet thick (Davidson, 1973).

The climate of the Tucson basin is semiarid and is characterized by hot summers and mild winters. The mean annual precipitation is about 12 in. at the lower altitudes and increases to $30 \mathrm{in}$. or more in the surrounding mountains. The Tucson basin has two distinct rainfall seasons, and about 50 percent of the annual precipitation occurs during the summer season. The summer rainfall is characterized by localized high-intensity and short-duration storms. The winter rainfall generally is less intense and of longer duration.

\section{Hydrology}

\section{Surface Water}

The Santa Cruz River and Rillito Creek are the major surface-water channels in the Tucson basin. The main tributaries to Rillito Creek include Tanque Verde Creek, Pantano Wash, and Alamo Wash. Rillito Creek flows about $12 \mathrm{mi}$ west-northwestward from the confluence of Pantano Wash and Tanque Verde Creek to the Santa Cruz River (fig. 2). Rillito Creek at Dodge Boulevard drains $871 \mathrm{mi}^{2}$ of mountains, desert, and approximately $34 \mathrm{mi}^{2}$ of urban area and is, for the most part, unregulated. Tanque Verde Creek at Sabino Canyon Road drains $219 \mathrm{mi}^{2}$ of mainly rural area, including mountainous areas in the northeastern part of the basin, and is dominated by winter flows. Pantano Wash at Broadway 


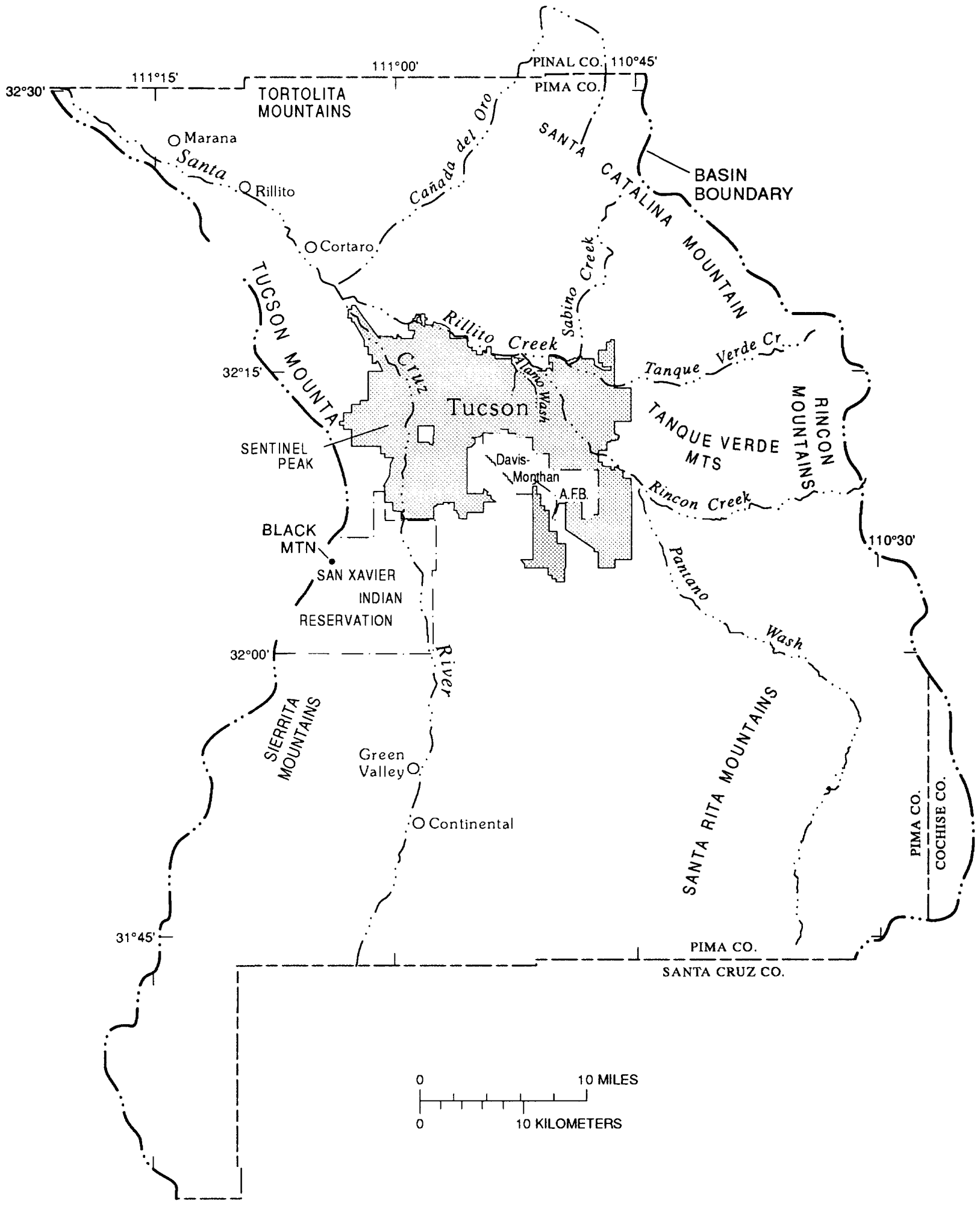

Figure 2. Location of Rillito Creek in the upper Santa Cruz drainage basin. 
Boulevard drains $599 \mathrm{mi}^{2}$ of the valley area in the southern and southeastem parts of the basin and is dominated by summer flows. Alamo Wash at Glenn Street drains $9.6 \mathrm{mi}^{2}$ of urban area. Rillito Creek and its tributaries are ephemeral, meaning that flow in the stream generally is in response to precipitation (Condes de la Torre, 1970).

Streamflow in the Rillito Creek and its tributaries are affected by the type of storm. Summer flows, which generally result from localized, high-intensity thunderstorms, are sudden and have high peak discharges, short durations, and high suspended-sediment concentrations. Winter flows, which generally result from more widespread frontal storms, generally have lower peak discharges, longer durations, and lower suspendedsediment concentrations (Matlock, 1965).

Streamflow is produced from rainfall and snowmelt originating in the Tanque Verde Creek and Rillito Creek watersheds. Flow in the Pantano and Alamo Washes generally consists of rainfall runoff. Runoff from local rainfall may last for several hours; however, streamflow from snowmelt may last for several weeks or more. Davidson (1973) calculated mean annual streamflow within the Tucson basin as 68,000 acre-ft for 1936-63. The average annual discharge passing the streamflow-gaging station, Rillito Creek near Tucson, was 11,660 acre-ft for 67 years of record for 1908-75 (U.S. Geological Survey, 1976).

\section{Ground Water}

According to Davidson (1973), recharge of the aquifer underlying the Tucson basin occurs primarily through streamflow infiltration and averages 51,000 acre- $\mathrm{ft} / \mathrm{yr}$; mountain-front recharge averages $31,000 \mathrm{acre}-\mathrm{ft} / \mathrm{yr}$; and subsurface inflow averages $17,000 \mathrm{acre}-\mathrm{ft} / \mathrm{yr}$. Other sources of recharge include return flows of water pumped for irrigation, public supply, and industrial use.

Ground-water levels in the basin fluctuate in response to high recharge in the stream channels and the pumping activity in the area (Camp Dresser and McKee, Inc., 1990). Streamflow data from the gaging station, Tanque Verde Creek at Tucson, and a hydrograph from well (D-13-14)26cbb2 about $3 \mathrm{mi}$ downstream indicate that water levels in the well respond to flow in the channel (fig. 3). Hydrographs for nine other wells in the study area (figs. 7-15) and tables 7-11 are presented in the "Basic Data" section at the back of the report. Water levels rise quickly when recharge occurs in Rillito Creek because of the high permeability of the sediments in the channel and the shallow depth to ground water (Camp Dresser and McKee, Inc., 1990). The depth to ground water ranged from about 10 to $120 \mathrm{ft}$ along Tanque Verde Creek, 225 to $350 \mathrm{ft}$ along Pantano Wash, 15 to $150 \mathrm{ft}$ along Rillito Creek, 60 to $150 \mathrm{ft}$ along the Santa Cruz River, and 125 to more than $350 \mathrm{ft}$ in the central part of the basin (City of Tucson, 1987-93).

\section{Artificial Recharge}

Artificial recharge is the planned recharge of an aquifer. Sources of water for artificial recharge can include storm runoff, imported river water, sewage effluent, irrigation water, and industrial wastewater (Wilson, 1985). Selection of a particular method for artificial recharge depends on land and water availability; physical, chemical, and biological composition of the recharge water, and hydrogeologic conditions of the area (Oaksford, 1985; Wilson, 1985).

Artificial recharge has been used in different parts of the world and in many locations in the United States to meet a number of water-resources management purposes (DeCook and Waterstone, 1987). Some purposes of artificial-recharge operations are (1) water conservation, (2) subsurface storage of water for the conjunctive management of surface-water and ground-water supplies, (3) control of floodwater and storm runoff, (4) control of water level and land subsidence, (5) creation of barriers to salt-water intrusion in coastal aquifers, (6) water-quality management, (7) purification of water through natural filtration, and (8) improvement of ground water by dilution (Richter and Chun, 1961; Wilson, 1985).

According to Wilson (1985), artificial recharge can be accomplished by water spreading, pits, and wells. Water spreading involves the release of water onto the surface of a basin where it percolates through the ground into the aquifer, the systems are either in-channel or off-channel. Recharge pits operate under the same principle as water-spreading basins but generally are excavated 


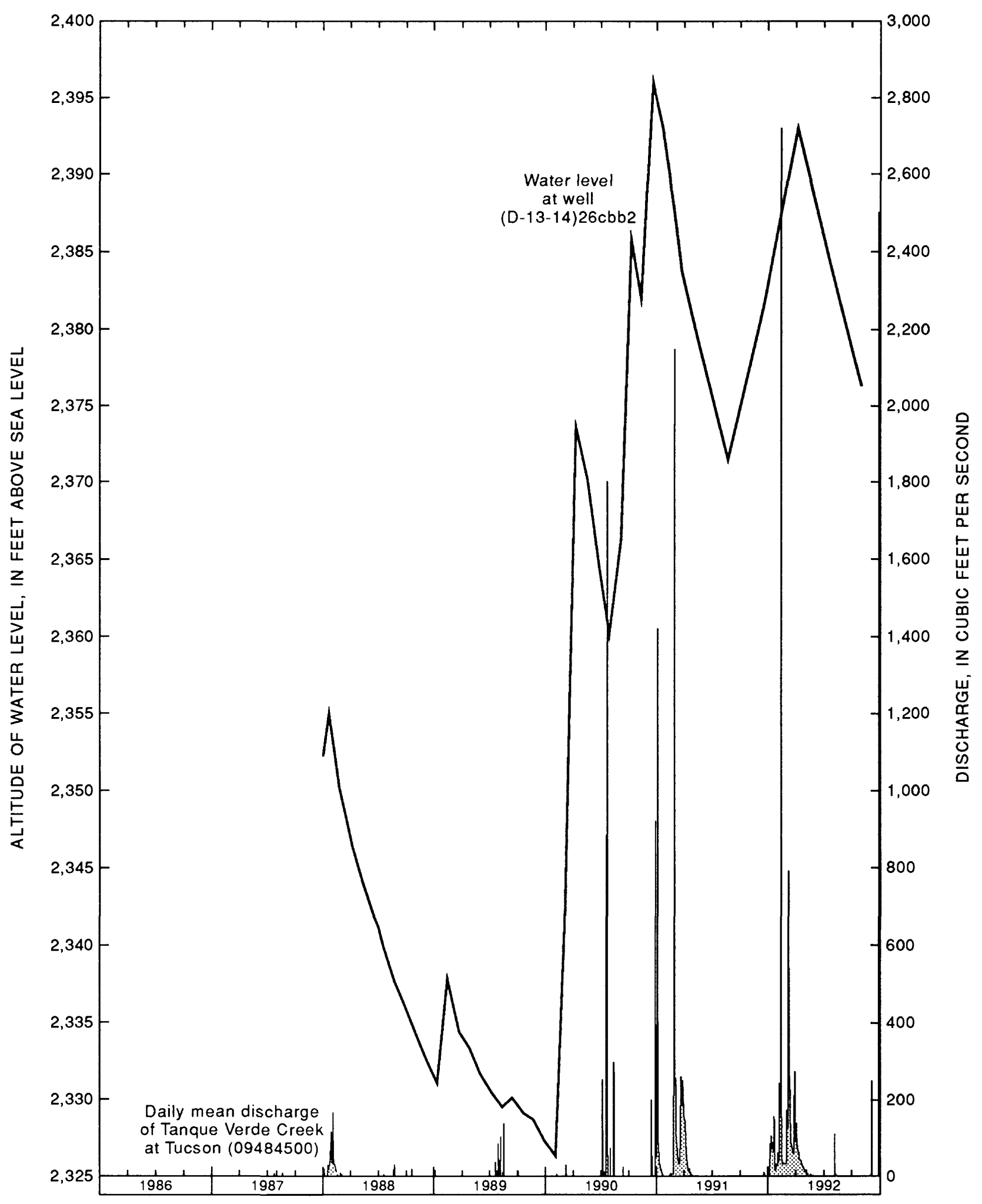

Figure 3. Flow in Tanque Verde Creek and water levels in observation well (D-13-14)26cbb2, 1986-92.

6 Quallty of Water and Chemlstry of Bottom Sediment In the RIIIlto Creek BasIn, Tucson Arizona, 1986-92 
to greater depth. Artificial recharge by well can be done by recharge wells, injection wells, and shafts or dry wells. The use of recharge wells involves the release of water into a well where it is carried directly into an unconfined aquifer by gravity. The use of an injection well involves the injection of water by extra pressure into the aquifer (generally confined). Recharge shafts or dry wells are bore holes constructed into permeable layers within the unsaturated zone. Water recharged through wells needs to be of good chemical and biological quality and generally low in suspended sediment (Brown and Signor, 1974).

The proposed Rillito Creek ground-water recharge project will utilize runoff entering a 1-mile reach of the Rillito Creek between Craycroft Road and Swan Road for infiltration and recharge purposes within the channel and excavated overbank areas. This proposed recharge can be done by water spreading and detention using an inflatable dam.

\section{Previous Studies}

Pashley (1966) described the structure and stratigraphy of the central, northern, and eastern parts of the Tucson basin. Davidson (1973) defined the geohydrology of the Tucson basin, and Anderson (1987) detailed the Cenozoic stratigraphy and geologic history of the Tucson basin. Anderson (1988) also reported on aquifer compaction, land subsidence, and earth fissures that are caused by a decline in ground-water levels. A regional study of the southwestern alluvial basins was done by Freethey and Anderson (1986), Robertson (1991), and Anderson and others (1992). The quality of water from Tucson's urban watersheds was studied by Dharmadhikari (1970). The quality of surface water and ground water in the Tucson area was reported by Laney (1972). The quality of sewage effluent recharged to the aquifer underlying the Santa Cruz River was investigated by Wilson and others (1975). The effect of urban runoff and its potential for ground-water pollution was studied by Mooradian (1980). Olson (1987) studied the potential of using dry wells for injection of urban stormwater in Tucson and its effect on ground-water quality. The effect of recharged effluent from the Nogales International Wastewater
Treatment Plant, Roger Road Wastewater Treatment Facility, and Ina Road Water Pollution Control Facility on the quality of ground water underlying the Santa Cruz River channel was studied by Schmidt and others (1989). The effect of silt-laden water on infiltration in alluvial channels of the Rillito Creek was studied by Matlock (1965). Infiltration in the main channels of the Tucson basin were investigated by Burkham (1970). Condes de la Torre (1970) studied the streamflow in the upper Santa Cruz River basin, Santa Cruz and Pima Counties. Studies related to stream-channel recharge in the Tucson basin were done by Keith (1981), Olson (1982), Kaddour (1983), and Katz (1987).

\section{APPROACH}

The quality and discharge of surface water were determined at three inflow sites and one outflow site for the recharge project area. Surfacewater samples were collected to determine possible occurrence and concentrations of contaminants. Ground-water samples were collected from 14 wells to determine general quality in the study area. Bottom-sediment samples also were collected at the four surface-water sites and analyzed to determine the presence and concentration of contaminants.

\section{METHODS OF DATA COLLECTION}

Discharge was determined from stage readings using rating curves developed from current-meter measurements. Surface-water samples were collected automatically and manually. Ground-water samples were collected by using submersible pumps. Bottom-sediment samples were collected by scooping.

\section{Surface-Water Discharge}

Runoff data are based on discharge measurements and stage records from four streamflow-gaging stations. The streamflowgaging stations-Tanque Verde Creek at Tucson; Pantano Wash at Broadway Boulevard, at Tucson; Alamo Wash at Tucson; and Rillito Creek at Dodge Boulevard, at Tucson-were run as 
stage-hydrograph stations (fig. 1). Discharge was either measured during sample collection, using current-meter measurements, or was determined by using recorded gage heights and previously drawn rating curves from stage-discharge relations.

The gaging stations on Tanque Verde and Rillito Creeks were equipped with a mercury manometer, a data-collection platform (DCP), and an analog recorder in a 4- by 4-foot walk-in shelter. The manometer measures stage by sensing pressure over an orifice near the streambed. Stage data are transferred to the DCP by an encoder. The DCP reads and stores data at 5-minute intervals and transmits the accumulated data to the Geostationary Operational Earth Satellite (GOES) every 4 hours. The data are retrieved from the satellite through the downlink station in Colorado and are transmitted to the computer system in Arizona.

A float-operated digital water-stage recorder was installed on the streamflow-gaging stations on Pantano and Alamo Washes. Stage data were recorded at 5-minute intervals. The gaging station on Pantano Wash has a gage house and a 24-inch-diameter stilling well, and the station on Alamo Wash has an in-bank stilling well and a 4by 4-foot walk-in shelter that houses a float-operated digital water-stage recorder.

The streamflow-gaging station on Tanque Verde Creek is on the left bank, as viewed from upstream, at the downstream side of the Sabino Canyon Road bridge. The channel is $320 \mathrm{ft}$ wide at the gaging station. The channel bed is natural and the banks are soil cement for a long distance upstream and downstream. The rating curve for Tanque Verde Creek was developed from 25 current-meter measurements between 1990 and 1991 and is considered good for flows greater than $300 \mathrm{ft}^{3} / \mathrm{s}$. Discharge computed for flows of less than $300 \mathrm{ft}^{3} / \mathrm{s}$ are significantly less accurate because small channels change constantly in the bed of the main channel. A stable relation between stage and discharge is not possible under such conditions.

The streamflow-gaging station on Pantano Wash is attached to a steel piling at the downstream side of the east-bound Broadway Boulevard bridge. The channel banks upstream from the gaging station are soil cement, and the bed is natural. The channel is $240 \mathrm{ft}$ wide at the gaging station. Because the rating curve for Pantano Wash was developed from only six current-meter measurements made since 1987, the rating curve is considered poor. The highest measured discharge was $4,470 \mathrm{ft}^{3} / \mathrm{s}$. More measurements are needed to establish a good rating curve.

The streamflow-gaging station on Alamo Wash is $270 \mathrm{ft}$ downstream from Glenn Street on the right bank. In 1986, the trapezoidal channel of Alamo Wash was lined with concrete from Glenn Street bridge to $330 \mathrm{ft}$ downstream. The channel is $60 \mathrm{ft}$ wide at the gaging station. The rating curve is fair and was developed from 10 current-meter measurements of less than $330 \mathrm{ft}^{3} / \mathrm{s}$ and one slope-area measurement of $4,000 \mathrm{ft}^{3} / \mathrm{s}$. More measurements of higher flows are needed to establish a good rating curve.

The streamflow-gaging station at Rillito Creek is on the right-hand side of the downstream bridge abutment at Dodge Boulevard. The channel upstream from the bridge has a natural sand bed and soil-cement banks. Downstream from the bridge, the bed and right bank are natural and the left bank is soil cement. The channel is $260 \mathrm{ft}$ wide at the gaging station. The north abutment was damaged twice in a 7-month period by large flows on July 24, 1990, and on January 6, 1991. Repairs to the abutment have made data collection more difficult. Large earthen berms were erected to protect the damaged abutment as it was being repaired. The earthen berms redirected flow away from the gaging station and isolated the station during periods of low flow. The gaging station, however, was not isolated during periods of medium flows. More measurements are needed to improve the rating at this site.

\section{Surface-Water Sampling}

From February 25, 1987, through March 13, 1992, samples of surface water were collected at four streamflow-gaging stations-Tanque Verde Creek at Tucson; Pantano Wash at Broadway Boulevard, at Tucson; Alamo Wash at Tucson; and Rillito Creek at Dodge Boulevard, at Tucson (fig. 1). Samples were collected by automatic and manual-sampling procedures. Automatic samples usually were collected at discharges greater than $1,000 \mathrm{ft}^{3} / \mathrm{s}$ that occurred late at night or early in the morning. Manual samples were collected at 
discharges of less than $300 \mathrm{ft}^{3} / \mathrm{s}$ that occurred during the day.

A Manning S-4501 automatic sampler was installed at each of the four stations. All wetted parts in the sampler are made of Teflon or glass to ensure that the samples were not contaminated by the sampling apparatus.

Automatic samplers at the Tanque Verde Creek and Rillito Creek stations were programmed to be activated when the stage exceeded a threshold value of $0.2 \mathrm{ft}$ in 2 minutes. A sample was collected every 5 minutes during a rising stage and every 10 minutes during a falling stage. The samplers at Pantano and Alamo Washes were activated at 2-minute intervals when the stage reached the threshold level of the 2-wire actuators. The sampler intake at each site was installed at least $1 \mathrm{ft}$ above the channel bed, and the threshold was set at least $0.2 \mathrm{ft}$ above the intake.

Samples were collected in twenty-four 350-milliliter (mL) glass bottles and were composited. A complete set of analyses for all constituent groups required a total of about 10 liters (L) of sample water. The maximum quantity of water that can be collected by the automatic samplers used for this investigation is about $7.7 \mathrm{~L}$; the average quantity of water collected was about 4.4 L. The average was less than the maximum possible because the bottles did not always fill completely and the durations of the flows were not always sufficient to allow for all 24 bottles to fill. Also, sample volume is lost when sediment is filtered out; therefore, when samples were collected with the automatic sampler, only selected analyses could be performed.

Large-volume samples were collected manually when possible to allow for a more complete set of analyses and to ensure a more representative sample. Samples were collected according to procedures described by the U.S Geological Survey (1977). Manual samples were collected using equal-width-increment methods and composited. The equal-width method requires equal spacing of several verticals across the cross section and an equal transit rate, both up and down, in all verticals. The width of increments to be sampled is determined by dividing the stream width by the number of vertical samples necessary to provide sufficient sample volume.
Manual sampling equipment consists of hand-held and cable-reel samplers. To prevent trace-metal contamination of the sample, the samplers are coated with epoxy paint and equipped with nylon nozzles and silicon gaskets. Where streams could be waded, a $\mathrm{DH}-48$ sampler was used to obtain samples. The DH-48 sampler consists of a streamlined aluminum casting 13 in. long that partly encloses the sample container. The container, typically a round pint glass bottle, is sealed against a rubber gasket recessed in the head cavity of the sampler by a hand-operated spring-tensioned pull-rod assembly at the tail of the sampler. As the sampling device is immersed, the sample enters the container and is collected through the intake nozzle. The sampler, including container, weighs 4.5 pounds and can sample to within 3.5 in. of the streambed (Guy and Norman, 1970).

When streams could not be waded, D-74 samplers were used to obtain samples. The D-74 sampler weighs 62 pounds and is designed for sampling with a cable-and-reel suspension. The D-74 sampler has a streamlined cast bronze body 24 in. long that completely encloses the sample container and is completely coated with epoxy paint to prevent trace-metal contamination of the sample. This sampler accommodates a quart glass bottle or, with the addition of an adaptor sleeve, a pint glass bottle can be used.

Surface-water samples were analyzed for properties and for concentrations of major ions, nutrients, trace elements, radionuclides, organochlorine pesticides, priority pollutants, volatile organic compounds, organic carbon, and oil and grease. Suspended-sediment samples were analyzed for sediment concentrations and for particle-size distribution.

All samples to be analyzed for dissolved constituents were passed through a 0.45 -micrometer pore size filter to remove suspended material. Samples for total constituents were discharged into sample bottles without being filtered. Water samples were collected by personnel of the U.S. Geological Survey in Tucson and processed in the field using methods described by the U.S. Geological Survey (1977).

Sample treatment and preservation were performed according to recommended methods of the U.S. Geological Survey (1985). Preservatives such as acids, bases, and metallic salts were added 
to water samples to retain dissolved constituents in solution, and to minimize changes due to chemical and biological activities (Ward and Harr, 1990). Some examples of preservation are refrigeration and additions of nitric acid, sulfuric acid, hydrochloric acid, mercuric chloride, and nitric acid-potassium dichromate.

Samples were analyzed for sediment concentration and particle-size distribution at USGS sediment laboratories in Iowa City, Iowa, and Vancouver, Washington. Radionuclide analysis of water samples was performed by a private laboratory under contract by the U.S. Geological Survey. All other analyses of water samples were performed by the U.S. Geological Survey National Water Quality Laboratory in Arvada, Colorado.

\section{Ground-Water Sampling}

Samples of water were collected from 14 wells from August 25, 1986, through March 28, 1989. The wells are within a 2-mile reach of Rillito Creek from the confluence of Tanque Verde Creek and Pantano Wash downstream to Dodge Boulevard (fig. 1). Production and monitoring wells were sampled in this study. Well-construction information for selected wells in the study area are presented in table 1. Dedicated pumps were used to collect samples from seven wells, and a portable submersible pump was used to collect samples from the remaining seven wells. Samples were collected by personnel of the Arizona Department of Water Resources. Before sampling, the wells were pumped until a minimum of three casing volumes of water were removed; specific conductance, $\mathrm{pH}$, dissolved-oxygen concentration, and temperature were continually monitored until stable. Ground-water samples were analyzed for properties and for the concentrations of major ions, nutrients, trace elements, radionuclides, organochlorine pesticides, priority pollutants, volatile organic compounds, organic carbon, and oil and grease. Ground-water samples were sent to the U.S. Geological Survey National Water Quality Laboratory in Arvada, Colorado. Ground-water samples were analyzed using the same procedures as those of surface-water samples.

\section{Bottom-Sediment Sampling}

From July 28, 1987, through February 20, 1992, samples of bottom sediment were collected from Tanque Verde Creek at Sabino Canyon Road, Pantano Wash at Broadway Boulevard, Alamo Wash at Glenn Street, and Rillito Creek at Dodge Boulevard. Samples were collected shortly after recession of runoff by personnel of the U.S. Geological Survey in Tucson. Samples were collected from the upper 2 in. of sediment using materials that would not be sources of additional contaminants. Plastic spoons and containers were used to collect samples for inorganic analyses and stainless-steel spoons and containers were used to collect samples for organic analyses. Samples were collected in equal-width increments across the channel, composited and mixed into a single sample, passed through a 500-micrometer-size sieve, and split into several sample containers in the field.

Samples were analyzed for nutrients, trace elements, radionuclides, organochlorine pesticides, priority pollutants, inorganic and organic carbon, and oil and grease. Particle-size distributions were determined on unsieved sediment from each site. Samples for particle-size distribution were analyzed using a wet-sieve method by U.S. Geological Survey sediment laboratories in Iowa City, Iowa, and Vancouver, Washington. Plastic containers were used for storage and shipment of bottom-sediment samples for analysis of inorganic constituents. Samples collected for the determination of organic compounds were stored and shipped in glass bottles. Samples for analysis of nutrients and organics were preserved by immediately chilling to $4^{\circ} \mathrm{C}$ to retard any chemical and (or) biological changes that may occur before analysis. Samples for analysis of trace elements and radionuclides required no preservation.

At the laboratory, samples for trace-elements analyses were air dried and then crushed and sieved through a 230 -mesh (63 micrometer) screen. The fine materials that passed through the screen were retained and analyzed. Analysis of bottomsediment samples for radionuclides was performed by a private laboratory under contract by the U.S. Geological Survey. Trace elements were analyzed by the U.S. Geological Survey, Geologic Division, Lakewood, Colorado. All other analyses of 
Tabie 1. Well-construction Information for selected wells, Rillito Creek basin

\begin{tabular}{|c|c|c|c|c|c|c|}
\hline $\begin{array}{c}\text { Weil } \\
\text { identliflcatlon } \\
\text { (D-13-14) }\end{array}$ & $\begin{array}{c}\text { Depth of well, } \\
\text { in feet }\end{array}$ & $\begin{array}{c}\text { Perforated zone, } \\
\text { in feet }\end{array}$ & $\begin{array}{l}\text { Diameter, in } \\
\text { inches }\end{array}$ & Casing type & $\begin{array}{c}\text { Date } \\
\text { instailed }\end{array}$ & Pump \\
\hline $26 \mathrm{bbb}$ & 200 & $90-195$ & 8 & Steel & 1960 & No \\
\hline $26 \mathrm{cbb} 2$ & 130 & $90-130$ & 6 & Steel & 1988 & No \\
\hline $26 \mathrm{cbb} 3$ & 80 & $45-80$ & 6 & Steel & 1988 & No \\
\hline $26 \mathrm{cdd}$ & 155 & $30-155$ & 12 & Steel & 1969 & Yes \\
\hline 26 dac2 & 100 & $50-100$ & 10 & Steel & 1971 & No \\
\hline $26 \mathrm{dcb} 2$ & 120 & $80-120$ & 6 & Steel & 1988 & No \\
\hline $26 \mathrm{dda} 2$ & 100 & $20-80$ & 12 & Steel & 1974 & Yes \\
\hline 27ada2 & 300 & $30-70$ & 12.75 & Steel & $\left({ }^{1}\right)$ & Yes \\
\hline $27 \mathrm{bdb} 4$ & 135 & $\left({ }^{1}\right)$ & 12 & Steel & 1954 & No \\
\hline $27 \mathrm{cda}$ & 320 & ${ }^{2} 160-225$ & $8-10$ & Steel & 1977 & Yes \\
\hline $27 \mathrm{ddc} 3$ & 240 & $200-240$ & 10 & Steel & 1936 & Yes \\
\hline 28dad & 150 & $50-130$ & 8 & Steel & ${ }^{2} 1935$ & Yes \\
\hline 34aaal & 135 & $40-130$ & 12 & Steel & 1948 & Yes \\
\hline $34 \mathrm{dcc} 2$ & 260 & $\left({ }^{1}\right)$ & 12 & Steel & 1951 & No \\
\hline
\end{tabular}

bed-material samples were performed by the U.S. Geological Survey, Geochemistry Laboratory, Arvada, Colorado.

\section{QUALITY OF WATER AND CHEMISTRY OF BOTTOM SEDIMENT}

This section contains a summary of physical and chemical data and interpretations of the data. Results of the analyses of all surface-water, ground-water, and bottom-sediment samples are given in tables 7-11 at the end of the report.

\section{Surface Water}

A summary was compiled from the several types of surface-water-quality data collected during the study-suspended sediment, properties, major ions, nutrients, trace elements, radionuclides, organochlorine pesticides, priority pollutants, organic carbon, and oil and grease (table 2). A summary of the results of analyses of constituent groups, such as organochlorine pesticides and priority pollutants, was determined only for those constituents detected. A summary of the results of analyses of volatile organic compounds was not compiled because none of the constituents were detected. Analytical results for all surface-water samples are presented in tables 7 and 8 at the end of the report. Although Rillito Creek is an ephemeral stream and is not used as a drinking-water supply, the water chemistry was compared with the U.S. Environmental Protection Agency (USEPA) primary and secondary drinking-water regulations and the State of Arizona drinking-water standards (State of Arizona, 1991).

Suspended sediment.-Concentrations of suspended sediment ranged from 22 to $36,700 \mathrm{mg} / \mathrm{L}$. Flow during the summer months generally contained higher concentrations of sediment than the flow during the rest of the year (table 7). Sediment concentrations generally tend to increase with an increase in streamflow. Suspended-sediment transport is of concern for several reasons. Suspended sediment can clog the channel bed during recharge, which reduces infiltration rates, and can serve as a transport mechanism for many inorganic, organic, and biological pollutants. 
Table 2. Summary of selected physical and chemical data for surface-water sites, Rillito Creek basin, February 25, 1987, through March 13,1992

$\left[\mathrm{mg} / \mathrm{L}\right.$, milligrams per liter, $\mu \mathrm{S} / \mathrm{cm}$, microsiemens per centimeter at $25^{\circ} \mathrm{C} ; \mathrm{ft}^{3} / \mathrm{s}$, cubic feet per second; ${ }^{\circ} \mathrm{C}$, degrees Celsius; $\mathrm{NTU}$, nephelometric-turbidity units; $\mu \mathrm{g} / \mathrm{L}$, micrograms per liter, $\mathrm{pCi} / \mathrm{L}$, picocuries per liter, <, less than. $\mathrm{DDD}$, dichlorodiphenyldichloroethane; $\mathrm{DDE}$, dichlorodiphenylethylene; DDT, dichlorodiphenyltrichloroethane]

\begin{tabular}{|c|c|c|c|c|}
\hline Constituent & $\begin{array}{c}\text { Number of } \\
\text { observations }\end{array}$ & Minimum & Maximum & Median \\
\hline \multicolumn{5}{|l|}{ Suspended sediment: } \\
\hline 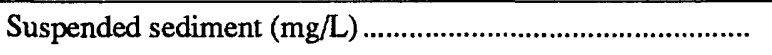 & 40 & 22 & 36,700 & 4,730 \\
\hline \multicolumn{5}{|l|}{ Properties: } \\
\hline 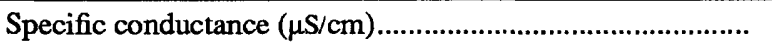 & 25 & 47 & 475 & 79 \\
\hline pH (units) & 28 & 6.5 & 9.2 & 8.3 \\
\hline 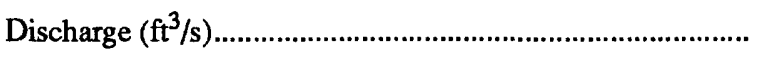 & 35 & 6 & 5,900 & 325 \\
\hline Water temperature $\left({ }^{\circ} \mathrm{C}\right)$ & 19 & 9 & 30.5 & 14 \\
\hline Turbidity (NTU) & 35 & 2.3 & 29,000 & 380 \\
\hline Dissolved oxygen $(\mathrm{mg} / \mathrm{L})$ & 7 & 6.2 & 11 & 6.6 \\
\hline Hardness as $\mathrm{CaCO}_{3}(\mathrm{mg} / \mathrm{L})$ & 35 & 15 & 150 & 35 \\
\hline Alkalinity as $\mathrm{CaCO}_{3}(\mathrm{mg} / \mathrm{L})$ & 26 & 12 & 97 & 31 \\
\hline Dissolved solids at $180^{\circ} \mathrm{C}(\mathrm{mg} / \mathrm{L})$ & 35 & 19 & 243 & 85 \\
\hline \multicolumn{5}{|l|}{ Major ions: } \\
\hline 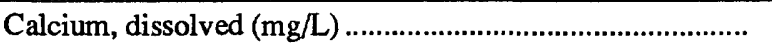 & 35 & 4.3 & 53 & 11 \\
\hline 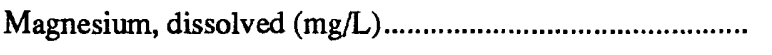 & 35 & .6 & 5.9 & 1.3 \\
\hline Sodium, dissolved $(\mathrm{mg} / \mathrm{L})$ & 35 & 1.5 & 14 & 4.5 \\
\hline Sodium adsorption ratio & 35 & .1 & .7 & .4 \\
\hline 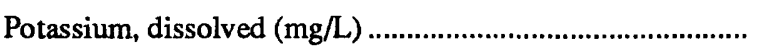 & 35 & .7 & 6.5 & 2.1 \\
\hline Bicarbonate as $\mathrm{HCO}_{3}(\mathrm{mg} / \mathrm{L})$ & 26 & 14 & 121 & 37 \\
\hline Sulfate, dissolved $(\mathrm{mg} / \mathrm{L})$ & 35 & 1.2 & 52 & 7.2 \\
\hline 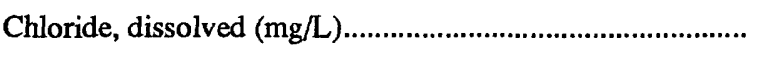 & 35 & .8 & 7.2 & 3.0 \\
\hline Fluoride, dissolved $(\mathrm{mg} / \mathrm{L})$ & 35 & $<.1$ & .6 & .1 \\
\hline 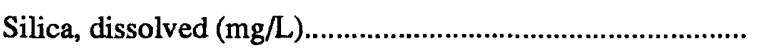 & 35 & 1.7 & 36 & 8.2 \\
\hline \multicolumn{5}{|l|}{ Nutrients $(\mathrm{mg} / \mathrm{L})$ : } \\
\hline 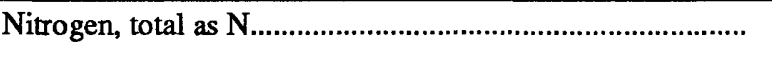 & 18 & .31 & 11 & 1.4 \\
\hline 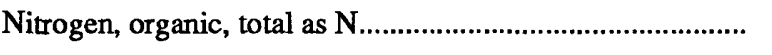 & 22 & .17 & 9.7 & .8 \\
\hline 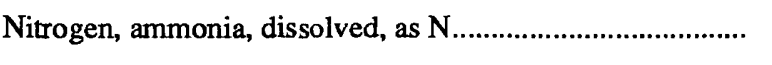 & 22 & $<.01$ & .56 & .06 \\
\hline Nitrogen, ammonia, total, as $\mathrm{N}$ & 22 & .01 & .97 & .08 \\
\hline Nitrogen, ammonia plus organic, total as $\mathrm{N}$ & 22 & .20 & 10 & 1.0 \\
\hline Nitrogen, nitrate, dissolved as $\mathrm{N}$ & 9 & .06 & .71 & .22 \\
\hline Nitrogen, nitrate, total as $\mathbf{N}$ & 15 & .08 & 1.13 & .37 \\
\hline Nitrogen, nitrite, dissolved as $\mathrm{N}$ & 13 & $<.01$ & .03 & .02 \\
\hline Nitrogen, nitrite, total as $\mathrm{N}$ & 22 & $<.01$ & .27 & .02 \\
\hline Nitrogen, nitrite plus nitrate, dissolved, as $\mathrm{N} . . . . . . . . . . . . . . . . . . . . . . . . .$. & 22 & $<.01$ & .98 & .27 \\
\hline
\end{tabular}


Table 2. Summary of selected physical and chemical data for surface-water sites, Rillito Creek basin, February 25, 1987, through March 13,1992-Continued

\begin{tabular}{|c|c|c|c|c|}
\hline Constituent & $\begin{array}{c}\text { Number of } \\
\text { observations }\end{array}$ & Minimum & Maximum & Median \\
\hline \multicolumn{5}{|l|}{ Nutrients $(\mathrm{mg} / \mathrm{L})-$ Continued } \\
\hline Nitrogen, nitrite plus nitrate, total as $\mathrm{N}$ & 22 & $<.01$ & 1.40 & .32 \\
\hline Phosphate, total as $\mathrm{PO}_{4}$ & 22 & .03 & 2.36 & .34 \\
\hline Orthophosphate, dissolved as $\mathrm{PO}_{4}$ & 21 & .03 & 1.20 & .42 \\
\hline 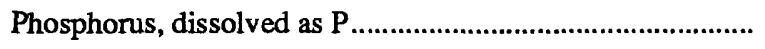 & 22 & .01 & .46 & .09 \\
\hline 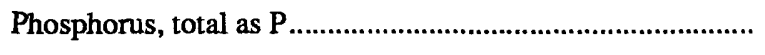 & 22 & .02 & 4.90 & .42 \\
\hline 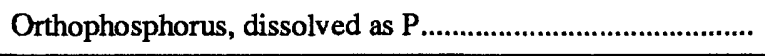 & 22 & $<.01$ & .38 & .08 \\
\hline \multicolumn{5}{|l|}{ Trace elements $(\mu \mathrm{g} / \mathrm{L})$ : } \\
\hline Aluminum, total recoverable & 42 & 470 & 550,000 & 89,500 \\
\hline 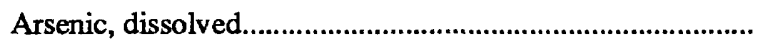 & 23 & $<1$ & 13 & 3 \\
\hline 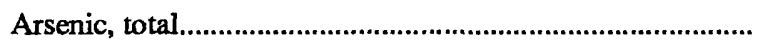 & 41 & $<1$ & 38 & 8 \\
\hline Barium, dissolved & 23 & $<100$ & 190 & 29 \\
\hline Barium, total recoverable & 42 & $<100$ & 10,000 & 1,000 \\
\hline Beryllium, total recoverable & 42 & $<10$ & 60 & $<10$ \\
\hline Boron, dissolved & 23 & $<10$ & 40 & 20 \\
\hline Cadmium, dissolved & 23 & $<1$ & 2 & $<1$ \\
\hline Cadmium, total recoverable & 42 & $<1$ & 12 & 2 \\
\hline 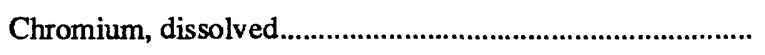 & 23 & $<1$ & 20 & $<1$ \\
\hline Chromium, total recoverable & 41 & $<1$ & 350 & 68 \\
\hline Cobalt, total recoverable & 42 & $<1$ & 180 & 30 \\
\hline Copper, dissolved . & 23 & $<10$ & 340 & 10 \\
\hline Copper, total recoverable & 39 & 2 & 2,500 & 260 \\
\hline Iron, total recoverable & 42 & 330 & 510,000 & 85,000 \\
\hline Lead, dissolved & 23 & $<1$ & $<5$ & 1 \\
\hline Lead, total recoverable & 40 & $<5$ & 1,900 & 155 \\
\hline Lithium, total recoverable & 42 & $<10$ & 1,600 & 110 \\
\hline Manganese, total recoverable & 42 & 20 & 49,000 & 4,900 \\
\hline Mercury, dissolved & 21 & $<.1$ & .2 & $<.1$ \\
\hline Mercury, total recoverable & 39 & $<.1$ & 2.8 & 3 \\
\hline 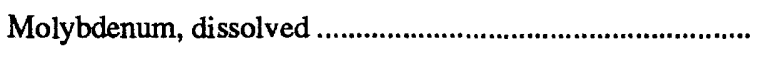 & 23 & $<1$ & 3 & 1 \\
\hline 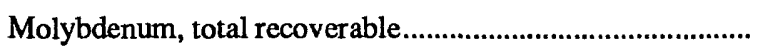 & 42 & $<1$ & 5 & 1 \\
\hline Nickel, dissolved & 23 & $<1$ & 4 & 1 \\
\hline Nickel, total recoverable & 42 & $<1$ & 700 & 97 \\
\hline 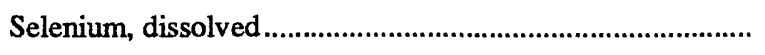 & 22 & $<1$ & 5 & $<1$ \\
\hline Selenium, total & 42 & $<1$ & 6 & $<1$ \\
\hline
\end{tabular}


Table 2. Summary of selected physical and chemical data for surface-water sites, Rillito Creok basin, February 25, 1987, through March 13,1992-Continued

\begin{tabular}{|c|c|c|c|c|}
\hline Constituent & $\begin{array}{l}\text { Number of } \\
\text { observations }\end{array}$ & Minimum & Maximum & Median \\
\hline \multicolumn{5}{|l|}{ Trace elements $(\mathrm{mg} / \mathrm{L})$ —Continued: } \\
\hline 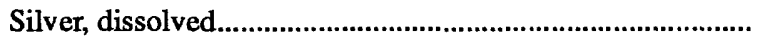 & 23 & $<1$ & 1 & $<1$ \\
\hline Silver, total recoverable & 42 & $<1$ & 4 & $<1$ \\
\hline 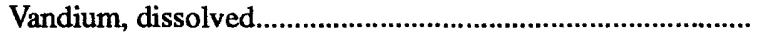 & 22 & 2 & 18 & 8 \\
\hline 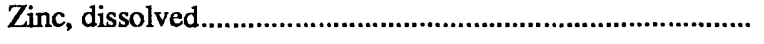 & 23 & $<3$ & 170 & $<10$ \\
\hline Zinc, total recoverable & 42 & $<10$ & 4,300 & 790 \\
\hline \multicolumn{5}{|l|}{ Radionuclides: } \\
\hline 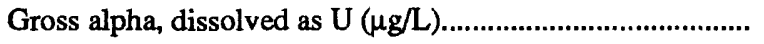 & 17 & $<.4$ & 4.4 & .8 \\
\hline Gross alpha, suspended as $\mathrm{U}(\mu \mathrm{g} / \mathrm{L})$ & 17 & $<.6$ & 1,500 & 93 \\
\hline 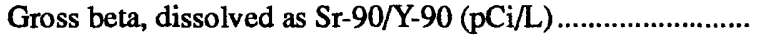 & 17 & .9 & 13 & 3.1 \\
\hline 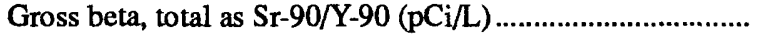 & 17 & $<.4$ & 910 & 97 \\
\hline Gross beta, dissolved as $\mathrm{Cs}-137(\mathrm{pCi} / \mathrm{L})$ & 17 & 1 & 17 & 4.1 \\
\hline 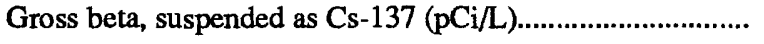 & 17 & $<.4$ & 1,000 & 110 \\
\hline \multicolumn{5}{|l|}{ Organochlorine pesticides, total recoverable $(\mu \mathrm{g} / \mathrm{L})$ : } \\
\hline 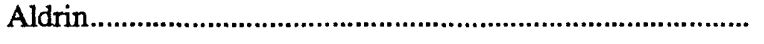 & 19 & $<.01$ & .02 & $<.01$ \\
\hline 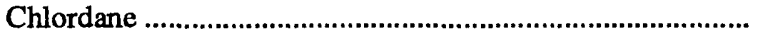 & 19 & $<.10$ & 1.50 & .01 \\
\hline DDD & 19 & $<.01$ & $<.1$ & $<.01$ \\
\hline 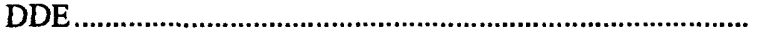 & 19 & $<.01$ & $<.1$ & $<.01$ \\
\hline DDT & 19 & $<.01$ & .1 & $<.01$ \\
\hline Dieldrin & 19 & $<.01$ & .19 & $<.01$ \\
\hline Endrin & 19 & $<.01$ & .01 & $<.01$ \\
\hline \multicolumn{5}{|l|}{ Priority pollutants, total recoverable $(\mu \mathrm{g} / \mathrm{L})$} \\
\hline 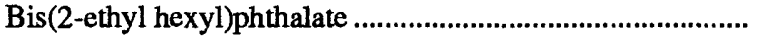 & 23 & $<5$ & $<13$ & $<5$ \\
\hline 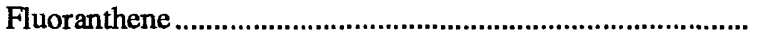 & 25 & $<5$ & 6 & $<5$ \\
\hline Pyrene & 25 & $<5$ & 6 & $<5$ \\
\hline \multicolumn{5}{|l|}{ Organic carbon and oil and grease $(\mathrm{mg} / \mathrm{L})$ : } \\
\hline 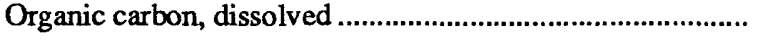 & 12 & 5.7 & 19 & 11.5 \\
\hline 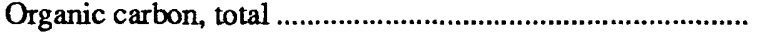 & 28 & 8.8 & 240 & 50.5 \\
\hline 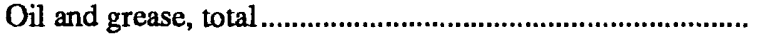 & 25 & $<1$ & 3 & $<1$ \\
\hline
\end{tabular}


Properties.-Specific conductance is the ability of water to conduct electrical current. Specific conductance generally was higher during the high flows that occurred in the summer months and lower during low flows that occurred during the winter and spring months from snowmelt runoff. Specific conductance ranged from $47 \mu \mathrm{S} / \mathrm{cm}$ in February 1992 at Tanque Verde Creek to $475 \mu \mathrm{S} / \mathrm{cm}$ in July 1990 at Rillito Creek.

The $\mathrm{pH}$ is defined as the negative log of the hydrogen-ion activity in water. When the $\mathrm{pH}$ is 7 , the water is said to be neutral; a pH of greater than 7 is basic (alkaline), and a pH of less than 7 is acidic. The data indicate that the water generally is alkaline. The $\mathrm{pH}$ of water for all sites ranged from 6.5 to 9.2 , and the median value was 8.3 for 28 samples. The median $\mathrm{pH}$ of 8.3 was within the USEPA secondary maximum contaminant level (SMCL) of 6.5 to 8.5 for drinking water (U.S. Environmental Protection Agency, 1993b).

Turbidity is an important indicator of water quality that relates to the penetration of light. Turbidity ranged from 2.3 at Tanque Verde Creek to 29,000 nephelometric-turbidity units (NTU) at Rillito Creek. Turbidity generally fluctuated seasonally and with discharge and was higher during the summer months and lower during the low-flow periods in the winter months. Increased turbidity in the summer probably was caused by suspended silts, clays, and organic particles in water.

Dissolved oxygen in surface water is derived from the atmosphere through aeration and is given off by aquatic plants in the process of photosynthesis. The solubility of oxygen in water is dependent on the partial pressure of oxygen in the air, water temperature, concentration of dissolved solids, and biological activity. As temperature and dissolved-solids concentration of the water increases, the saturation concentration of dissolved oxygen decreases. Dissolved-oxygen concentrations ranged from 6.2 at Alamo Wash to $11 \mathrm{mg} / \mathrm{L}$ at Tanque Verde Creek.

Hardness is a measure of the relative amount of certain ions in water, mainly calcium and magnesium, that form insoluble precipitates with soap. According to Hem (1989), water with a hardness of less than $60 \mathrm{mg} / \mathrm{L}$ as calcium carbonate $\left(\mathrm{CaCO}_{3}\right)$ is soft, 61 to $120 \mathrm{mg} / \mathrm{L}$ is moderately hard, 121 to $180 \mathrm{mg} / \mathrm{L}$ is hard, and more than $180 \mathrm{mg} / \mathrm{L}$ is very hard. Data indicate that the surface water in this study was soft to hard. Hardness values generally ranged from 15 to $81 \mathrm{mg} / \mathrm{L}$ as $\mathrm{CaCO}_{3}$ except in two samples from Alamo Wash and Rillito Creek, which were 140 and $150 \mathrm{mg} / \mathrm{L}$, respectively.

Alkalinity is a measure of the capacity of water to neutralize acid. Alkalinity of water is due primarily to the presence of bicarbonate, carbonate, and hydroxide ions. Alkalinity in filtered samples ranged from $12 \mathrm{mg} / \mathrm{L}$ (as $\mathrm{CaCO}_{3}$ ) at Tanque Verde Creek to $97 \mathrm{mg} / \mathrm{L}$ at Rillito Creek and is expressed in terms of an equivalent amount of $\mathrm{CaCO}_{3}$.

Dissolved solids is a general term used to describe the mineral content of water. Dissolved solids consist primarily of calcium, magnesium, sodium, potassium, bicarbonate, sulfate, chloride, and nitrate. At Rillito Creek, dissolved-solids concentrations ranged from 19 to $243 \mathrm{mg} / \mathrm{L}$. Surface water typically had low concentrations of dissolved solids, which were below the SMCL of $500 \mathrm{mg} / \mathrm{L}$ for drinking water.

Major ions.-Calcium, magnesium, and sodium are common constituents in most natural waters and result from the dissolution of many rock minerals. Concentrations of dissolved calcium, magnesium, and sodium in water at all sites in this study generally were low. The highest concentrations of calcium were detected at Rillito Creek (46 mg/L) and Alamo Wash $(53 \mathrm{mg} / \mathrm{L})$. Maximum concentrations of magnesium and sodium were detected in samples from Rillito Creek and ranged from 0.8 to $5.9 \mathrm{mg} / \mathrm{L}$ and 3.9 to $14 \mathrm{mg} / \mathrm{L}$, respectively. Potassium concentrations ranged from 0.7 to $6.5 \mathrm{mg} / \mathrm{L}$ in the study area.

The sodium-adsorption ratios, which generally indicate the proportion of sodium over calcium and magnesium, were small at all sites and ranged from 0.1 to 0.7. According to Ayers and Westcot (1986), infiltration rates have been reduced on agricultural land by application of water that had a sodium-adsorption ratio greater than 3 . Sodium tends to cause swelling and dispersion of clays, which clog and reduce permeability in the soil (Hillel, 1980).

Dissolved bicarbonate concentrations $\left(\mathrm{HCO}_{3}\right)$ ranged from 14 at Tanque Verde Creek to $121 \mathrm{mg} / \mathrm{L}$ at Rillito Creek. The highest dissolved sulfate concentration of $52 \mathrm{mg} / \mathrm{L}$ was detected in July 1988 at Rillito Creek. Low concentrations of dissolved 
chloride and fluoride were detected in the study area and ranged from 0.8 to $7.2 \mathrm{mg} / \mathrm{L}$ and less than 0.1 to $0.6 \mathrm{mg} / \mathrm{L}$, respectively. Concentrations of sulfate and chloride were below the USEPA SMCL's of $250 \mathrm{mg} / \mathrm{L}$. Concentrations of fluoride were below the maximum contaminant level (MCL) of $4 \mathrm{mg} / \mathrm{L}$ (U.S. Environmental Protection Agency, 1993a). Sulfate occurs naturally in water from the leaching of gypsum, chloride occurs in igneous rock, and fluoride occurs in igneous and sedimentary rocks (Hem, 1989). The concentration of dissolved silica generally was low at all four sites. The maximum silica concentration was 36 $\mathrm{mg} / \mathrm{L}$ in one sample from Alamo Wash. The trilinear diagram illustrates the relative proportions of major ions in surface-water samples (fig. 4). The water is primarily a calcium and bicarbonate type.

Nutrients.-Nitrogen occurs in several forms including molecular nitrogen, ammonia, organic nitrogen, nitrate, and nitrite (Moore, 1991). Nitrogen compounds in surface water originate from both natural and anthropogenic sources (Marron and others, 1989; Moore, 1991). Natural sources of nitrogen are found in soil or biological material, and anthropogenic sources include fertilizers, sewage, and animal wastes (Hem, 1989; Marron and others, 1989; Moore, 1991). Concentrations of nitrogen, organic nitrogen, ammonia, nitrate, and nitrite generally were low for all four sampling sites.

Total nitrogen is a measure of organic and inorganic forms of dissolved and suspended nitrogen. The maximum concentrations of total nitrogen (as $\mathrm{N}$ ) were $11 \mathrm{mg} / \mathrm{L}$ at Tanque Verde Creek, $3.3 \mathrm{mg} / \mathrm{L}$ at Pantano Wash, $3.2 \mathrm{mg} / \mathrm{L}$ at Alamo Wash, and $4.4 \mathrm{mg} / \mathrm{L}$ at Rillito Creek. The total organic nitrogen (as $\mathrm{N}$ ) in water was detected in the greatest concentration of any nitrogen species and ranged from 0.17 to $9.7 \mathrm{mg} / \mathrm{L}$ at Tanque Verde Creek, from 0.63 to $2.6 \mathrm{mg} / \mathrm{L}$ at Pantano Wash, from 0.26 to $1.9 \mathrm{mg} / \mathrm{L}$ at Alamo Wash, and 0.52 to $3.0 \mathrm{mg} / \mathrm{L}$ at Rillito Creek. The concentrations of total ammonia (as $\mathrm{N}$ ) ranged from 0.09 to $0.59 \mathrm{mg} / \mathrm{L}$ at Alamo Wash and 0.07 to $0.97 \mathrm{mg} / \mathrm{L}$ at Rillito Creek. The maximum concentrations of total nitrate $(1.13 \mathrm{mg} / \mathrm{L})$ and total nitrite as $\mathrm{N}$ $(0.27 \mathrm{mg} / \mathrm{L})$ were detected at Rillito Creek. Low concentrations of dissolved ammonia, nitrate, and nitrite were detected in the study area and ranged from less than 0.01 to $0.56 \mathrm{mg} / \mathrm{L}$, from 0.06 to
$0.71 \mathrm{mg} / \mathrm{L}$ and less than 0.01 to $0.03 \mathrm{mg} / \mathrm{L}$, respectively. Concentrations of nitrate plus nitrite (as $\mathrm{N}$ ) and nitrite (as $\mathrm{N}$ ) were below the USEPA MCL for drinking water of $10 \mathrm{mg} / \mathrm{L}$ and $1 \mathrm{mg} / \mathrm{L}$, respectively

Sources of phosphate in water include fertilizers, animal metabolic waste, and weathering of igneous rocks (Hem, 1989). According to Hem (1989), concentrations of phosphate ions in most natural waters rarely exceed a few tenths or hundredths of a milligram per liter owing to the adsorption of phosphate ions by metal oxides. Concentrations of phosphorous and phosphate were low at all four sampling sites.

The maximum concentration of total phosphate (as $\left.\mathrm{PO}_{4}\right)$ was $2.36 \mathrm{mg} / \mathrm{L}$ at Tanque Verde Creek. The highest concentration of total phosphorus (as P) was $4.90 \mathrm{mg} / \mathrm{L}$ at Alamo Wash. Concentrations of dissolved orthophosphorus (as P) generally were small in the study area and ranged from less than 0.01 to $0.38 \mathrm{mg} / \mathrm{L}$ at Tanque Verde Creek.

Trace elements.-The concentrations of elements in natural water are the result of natural weathering and erosion of rocks and soils. According to Hem (1989), chemical properties can be more important in controlling concentrations of an element in water than is the average abundance of the elements in the rock materials. Concentrations of dissolved and total trace elements generally were highest during the high flows caused by summer rainstorms and lowest during low flows that generally occurred during the winter and spring months as a result of snowmelt runoff.

The highest measured concentrations of total recoverable aluminum $(550,000 \mu \mathrm{g} / \mathrm{L})$, barium $(10,000 \mu \mathrm{g} / \mathrm{L})$, beryllium $(60 \mu \mathrm{g} / \mathrm{L})$, cadmium (12 $\mu \mathrm{g} / \mathrm{L})$, chromium $(350 \mu \mathrm{g} / \mathrm{L})$, copper $(2,500 \mu \mathrm{g} / \mathrm{L})$, mercury $(2.8 \mu \mathrm{g} / \mathrm{L})$, iron $(510,000 \mu \mathrm{g} / \mathrm{L})$, lead $(1,900 \mu \mathrm{g} / \mathrm{L})$, manganese $(49,000 \mu \mathrm{g} / \mathrm{L})$, and nickel $(700 \mu \mathrm{g} / \mathrm{L})$ were detected in surface-water samples. The State of Arizona MCL's for total recoverable barium $(1,000 \mu \mathrm{g} / \mathrm{L})$, chromium $(50 \mu \mathrm{g} / \mathrm{L})$, and lead $(50 \mu \mathrm{g} / \mathrm{L})$ were exceeded in most of the samples at all four sampling sites (State of Arizona, 1991). The State of Arizona MCL's for cadmium $(10 \mu \mathrm{g} / \mathrm{L})$ and mercury $(2 \mu \mathrm{g} / \mathrm{L})$ were exceeded in one sample from Tanque Verde Creek. None of the dissolved constituent values exceeded the State of Arizona 


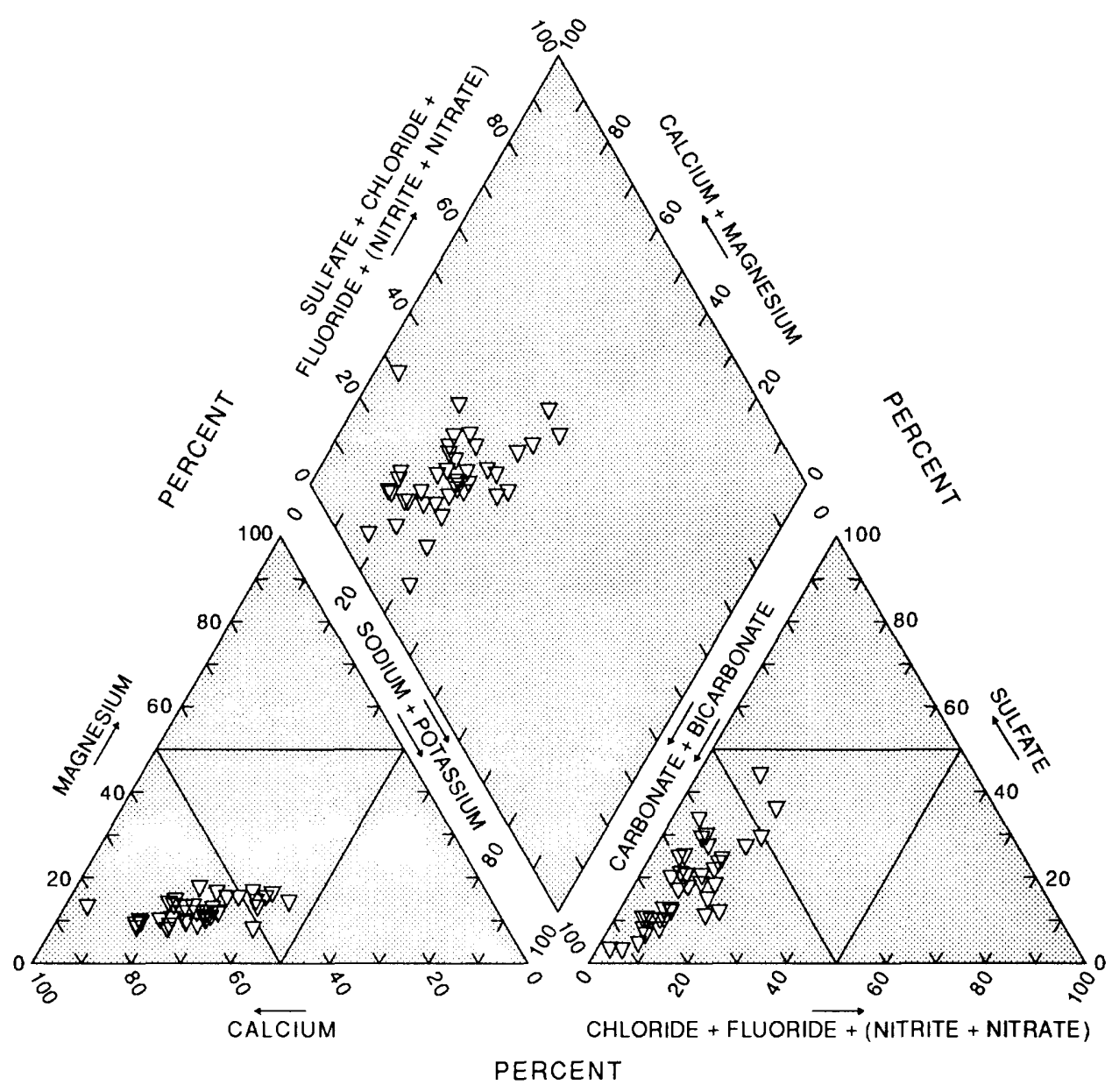

Figure 4. Relative compositions of surface water, in percent, Rillito Creek basin, 1987-92.

MCL's. The USEPA SMCL's for total recoverable aluminum $(50 \mu \mathrm{g} / \mathrm{L})$, manganese $(50 \mu \mathrm{g} / \mathrm{L})$, and iron $(300 \mu \mathrm{g} / \mathrm{L})$ were exceeded in most of the samples at the four sites.

Samples to be analyzed for dissolved trace elements were passed through a 0.45 -micrometer pore-size filter that removed most of the suspended material and resulted in significantly lower trace-element concentrations. A sample that is to be analyzed for total recoverable constituents is not filtered, and therefore contains the same amount of sediment by volume as the suspended-sediment sample. Concentrations of total recoverable chromium, nickel, and zinc correlated with suspended-sediment concentrations (fig. 5).
Trace metals tend to adhere to the surface of sediment particles, and a strong positive correlation exists between decreasing grain size and increasing trace-element concentration (Horowitz, 1985). According to Horowitz (1985), the metal ions are attracted to the surface of colloidal material; therefore, sediments with large surface-area to mass ratios such as clay particles, are the best adsorbers. Surfaces of the clay particles are negatively charged and tend to adsorb to the positively charged cations in the water (Dunn and others, 1980). Clay-sized particles have proportionally large surface areas and are measured in square meters per gram as opposed to sand-sized particles with surface area commonly measured in square centimeters per gram (Horowitz, 1985). 


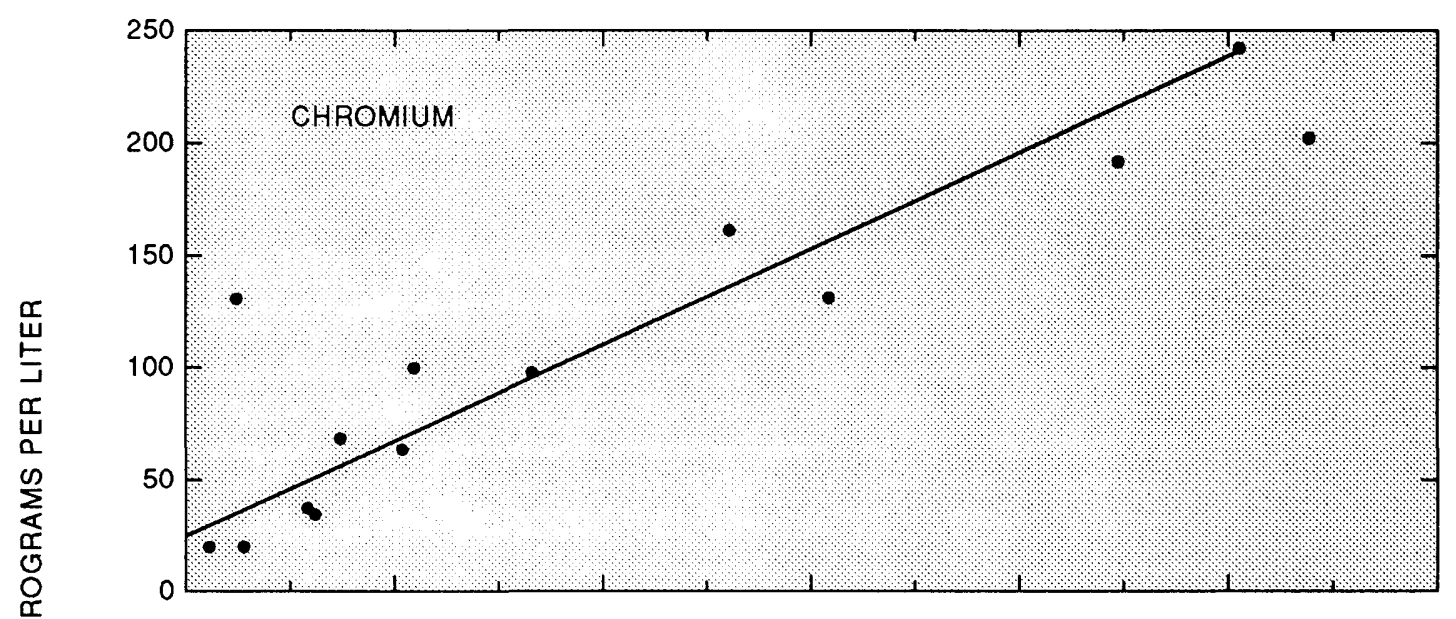

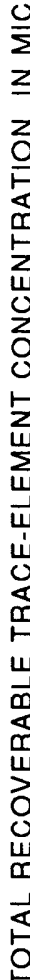

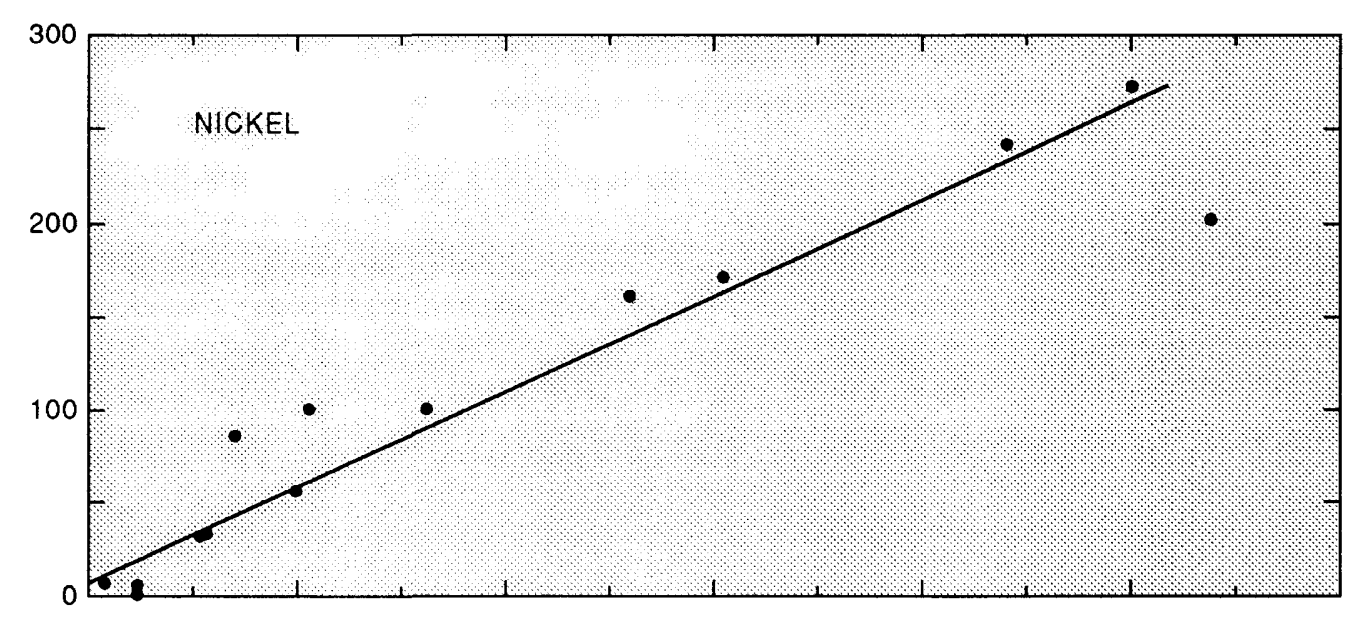

E

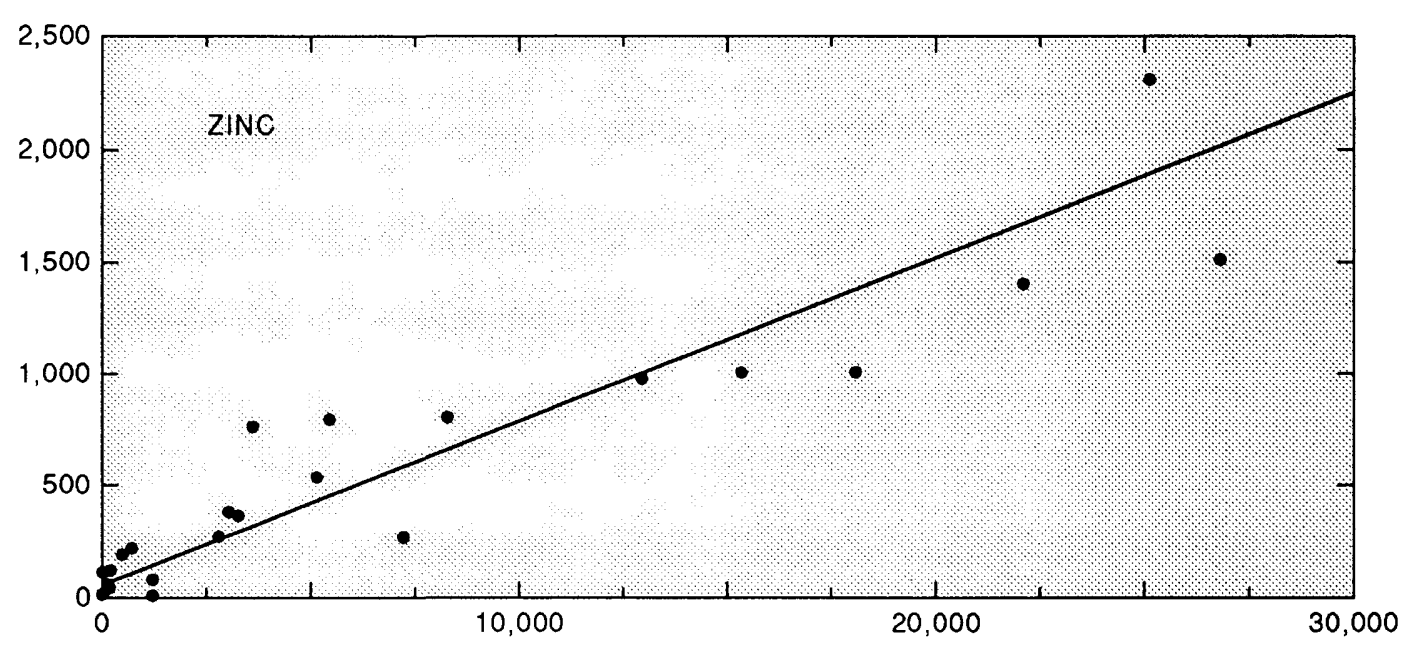

SUSPENDED-SEDIMENT CONCENTRATION, IN MILLIGRAMS PER LITER

Figure 5. Relation between selected total recoverable trace-element concentration and suspendedsediment concentration in surface-water samples, Rillito Creek basin. 
Radionuclides.-Radionuclides in natural water are the result of disintegration of high-atomic-weight isotopic elements. Seventeen surface-water samples were analyzed for gross alpha and gross beta activities. Dissolved and suspended gross alpha and gross beta activities were higher in samples from high flows that occurred in the summer months and lower in samples from low flows that generally occurred during the winter and spring months from snowmelt runoff. As a result of sorptive processes, radionuclides in surface waters are bound predominantly to stream sediments (Sayre and others, 1963). Gross alpha and gross beta activities in samples of suspended sediment were extremely variable throughout the study area.

Dissolved gross alpha activity (as natural uranium $U$ ) and suspended gross alpha activity (as U) were highest at Pantano Wash-4.4 and $1,500 \mu \mathrm{g} / \mathrm{L}$, respectively. The highest activity of dissolved gross beta was detected at Tanque Verde Creek (13 pCi/L). Activity of suspended gross beta (as Sr-90/Y-90) ranged from less than 0.4 to $320 \mathrm{pCi} / \mathrm{L}$ at Tanque Verde Creek, 23 to $910 \mathrm{pCi} / \mathrm{L}$ at Pantano Wash, 6.8 to $110 \mathrm{pCi} / \mathrm{L}$ at Alamo Wash, and 5.2 to $360 \mathrm{pCi} / \mathrm{L}$ at Rillito Creek. The highest activity of gross beta as cesium-137 (Cs-137) was detected in Tanque Verde Creek (17 pCi/L). Activity of suspended gross beta (as Cs-137) ranged from less than 0.4 to $340 \mathrm{pCi} / \mathrm{L}$ at Tanque Verde Creek, 25 to $1,000 \mathrm{pCi} / \mathrm{L}$ at Pantano Wash, 7.5 to $120 \mathrm{pCi} / \mathrm{L}$ at Alamo Wash, and 5.7 to 390 $\mathrm{pCi} / \mathrm{L}$ at Rillito Creek.

The unadjusted median value of $93 \mu \mathrm{g} / \mathrm{L}$ for suspended gross alpha activity (as $\mathrm{U}$ ) that converts to $63.2 \mathrm{pCi} / \mathrm{L}$ (assuming a 0.68 conversion factor) could exceed the State of Arizona MCL for drinking water of $15 \mathrm{pCi} / \mathrm{L}$ for adjusted gross alpha (minus uranium and radon). Uranium and radon need to be measured separately to determine compliance. Dissolved gross alpha activities (as U) did not exceed the USEPA MCL for drinking water. USEPA or State of Arizona MCL's do not exist for gross beta. Activities of dissolved gross alpha and dissolved gross beta were significantly lower than the activities of suspended gross alpha and suspended gross beta because suspended material was removed by filtration.

Organochlorine pesticides.-Multiple organochlorine pesticides were detected in samples collected at Alamo Wash and Rillito Creek. Chlordane was the only constituent detected at all four sampling sites. Pesticides that were detected at Alamo Wash were aldrin, chlordane, dichlorodiphenyldichloroethane (DDD), dichlorodiphenylethylene (DDE), dichlorodiphenyltrichloroethane (DDT), dieldrin, and endrin. Chlordane and dieldrin were detected at Rillito Creek; chlordane was detected at Tanque Verde Creek and Pantano Wash.

The maximum aldrin concentration of $0.02 \mu \mathrm{g} / \mathrm{L}$ was detected in a sample from Alamo Wash. Chlordane was detected at all four sampling sites; concentrations ranged from less than 0.1 to $0.2 \mu \mathrm{g} / \mathrm{L}$ at Tanque Verde Creek, less than 0.01 to $0.2 \mu \mathrm{g} / \mathrm{L}$ at Pantano Wash, less than 0.1 to $1.5 \mu \mathrm{g} / \mathrm{L}$ at Alamo Wash, and less than 0.1 to $0.1 \mu \mathrm{g} / \mathrm{L}$ at Rillito Creek. Concentrations of DDD, DDE, DDT, and endrin were detected only in samples from Alamo Wash; the maximum concentrations detected were $0.03,0.05,0.01$, and $0.01 \mu \mathrm{g} / \mathrm{L}$, respectively. Dieldrin was detected only in samples from Alamo Wash and Rillito Creek, and the maximum levels were 0.19 and $0.02 \mu \mathrm{g} / \mathrm{L}$, respectively. Concentrations of chlordane and endrin were below the USEPA MCL of $2 \mu \mathrm{g} / \mathrm{L}$. Some of the pesticides in the water probably are the result of frequent use of chemicals to control weeds and insects in nearby urban and agricultural areas.

Priority pollutants. - The chemicals bis(2ethylhexyl)phthalate and fluoranthene were detected in three of nine samples from Alamo Wash and in two of six samples from Rillito Creek. The maximum concentrations of bis(2-ethylhexyl)phthalate were $10 \mu \mathrm{g} / \mathrm{L}$ at Alamo Wash and $11 \mu \mathrm{g} / \mathrm{L}$ at Rillito Creek. Fluoranthene was detected in only 5 of 10 samples from Alamo Wash and the maximum concentration was $6 \mu \mathrm{g} / \mathrm{L}$. Pyrene also was detected in 4 of 10 samples from Alamo Wash, and concentrations ranged from less than 5 to $6 \mu \mathrm{g} / \mathrm{L}$. Distribution of the priority pollutants probably is the result of a higher intensity of urbanization and type of land use within the Alamo Wash and Rillito Creek watersheds.

Volatile organic compounds.-Volatileorganic compounds (VOC's) were not detected in samples from the four sampling sites. The detection limits for the VOC's were 1,3 , or $5 \mu \mathrm{g} / \mathrm{L}$.

Organic carbon.-Organic carbon in natural water is derived from soil and plants (Thurman, 
1985). Dissolved and total organic carbon were detected at all four sites. Concentrations of dissolved organic carbon ranged from 5.7 at Pantano Wash to $19 \mathrm{mg} / \mathrm{L}$ at Tanque Verde Creek. Concentrations of total organic carbon were higher during the high flows that occurred in the summer months and lower during low flows that generally occurred during the winter and spring months from snowmelt runoff. Total organic carbon ranged from 8.8 to $240 \mathrm{mg} / \mathrm{L}$ at Tanque Verde Creek, 30 to $150 \mathrm{mg} / \mathrm{L}$ at Pantano Wash, 14 to $93 \mathrm{mg} / \mathrm{L}$ at Alamo Wash, and 19 to $210 \mathrm{mg} / \mathrm{L}$ at Rillito Creek.

Oil and grease.-Oil and grease were detected at all sampling sites except Pantano Wash and ranged from less than 1 to $2 \mathrm{mg} / \mathrm{L}$ at Tanque Verde Creek, less than 1 to $3 \mathrm{mg} / \mathrm{L}$ at Alamo Wash, and less than 1 to $2 \mathrm{mg} / \mathrm{L}$ at Rillito Creek. Oil and grease in surface water probably was the result of rainfall runoff from roads and parking lots.

\section{Ground Water}

A summary was compiled from the following types of ground-water data that were collected during the study-properties, major ions, nutrients, trace elements, radionuclides, organic carbon, and oil and grease (table 3). Organochlorine pesticides, priority pollutants, and volatile organics were not detected in the samples. Results of the analyses of all ground-water samples are presented in table 9 at the end of the report.

Properties.-Specific conductance in ground water ranged from $215 \mu \mathrm{S} / \mathrm{cm}$ at well (D-13-14)27bdb4 to $720 \mu \mathrm{S} / \mathrm{cm}$ at well (D-13-14)26bbb. pH ranged from 6.7 to 7.9 and was within the SMCL range of 6.5 to 8.5 . Turbidity generally ranged from 0.1 to 3.8 NTU; however, samples from well (D-13-14) 26dcb2 and (D-13-14)26bbb were 25 and 90 NTU, respectively. Turbidity in ground water probably is due to well construction. Dissolved-oxygen concentrations ranged from $2.6 \mathrm{mg} / \mathrm{L}$ at well (D-13-14)26dac2 to $10.4 \mathrm{mg} / \mathrm{L}$ at well (D-13-14)28dad. The data indicate that the ground water is soft to very hard. Hardness concentrations ranged from $59 \mathrm{mg} / \mathrm{L}$ (as $\mathrm{CaCO}_{3}$ ) at well (D-13-14)34aaal to $390 \mathrm{mg} / \mathrm{L}$ at well (D-13-14)26bbb. Laboratory-measured alkalinities for unfiltered samples ranged from $83 \mathrm{mg} / \mathrm{L}$ at well
(D-13-14)27bdb4 to $219 \mathrm{mg} / \mathrm{L}$ at well (D-13-14)34dcc2. Dissolved-solids concentrations ranged from $132 \mathrm{mg} / \mathrm{L}$ at well (D-13-14)27bdb4 to $689 \mathrm{mg} / \mathrm{L}$ at well (D-13-14)26bbb. The maximum concentration of $689 \mathrm{mg} / \mathrm{L}$ in one sample from well (D-13-14)26bbb exceeded the SMCL of $500 \mathrm{mg} / \mathrm{L}$.

Major ions.-Concentrations of dissolved calcium $(120 \mathrm{mg} / \mathrm{L})$ and magnesium $(21 \mathrm{mg} / \mathrm{L})$ were highest in samples from well (D-13-14)26bbb. Dissolved sodium concentrations ranged from $7.2 \mathrm{mg} / \mathrm{L}$ at well (D-13-14)27bdb4 to $55 \mathrm{mg} / \mathrm{L}$ at well (D-13-14)34dcc2. Sodium-adsorption ratios ranged from 0.3 to 2 . Concentrations of dissolved potassium generally were low and ranged from $0.9 \mathrm{mg} / \mathrm{L}$ at well (D-13-14)34aaal to $3.1 \mathrm{mg} / \mathrm{L}$ at well (D-13-14)26bbb.

Dissolved bicarbonate concentrations ranged from $101 \mathrm{mg} / \mathrm{L}$ (as $\mathrm{CaCO}_{3}$ ) at well (D-13-14)27bdb4 to $267 \mathrm{mg} / \mathrm{L}$ at well (D-13-14)34dcc2. Dissolved sulfate concentrations ranged from $10 \mathrm{mg} / \mathrm{L}$ at well (D-13-14)28dad to 42 $\mathrm{mg} / \mathrm{L}$ at well (D-13-14)34dcc2. Dissolved chloride concentrations generally ranged from 2 to $43 \mathrm{mg} / \mathrm{L}$; however, samples from well (D-13-14)26bbb had concentrations of 97 and $180 \mathrm{mg} / \mathrm{L}$. Dissolved fluoride concentrations ranged from 0.1 to $0.6 \mathrm{mg} / \mathrm{L}$. Maximum concentrations of dissolved sulfate, chloride, and fluoride did not exceed the SMCL's of $250 \mathrm{mg} / \mathrm{L}$ for sulfate and chloride and the MCL of $4 \mathrm{mg} / \mathrm{L}$ for fluoride. Dissolved silica concentrations ranged from $17 \mathrm{mg} / \mathrm{L}$ at well (D-13-14)27bdb4 to $43 \mathrm{mg} / \mathrm{L}$ at well (D-13-14)26bbb. The water is primarily a calcium sodium and bicarbonate type (fig. 6).

Nutrients.-Concentrations of total nitrogen (as $\mathrm{N}$ ) ranged from $0.7 \mathrm{mg} / \mathrm{L}$ at well (D-13-14)26dda2 to $25 \mathrm{mg} / \mathrm{L}$ at well (D-13-14)26bbb. Total nitrate (as $\mathrm{N}$ ) in ground water was detected in the highest concentration of any nitrogen species. Organic nitrogen is converted to ammonia by bacteria and then under aerobic conditions the ammonia is oxidized to nitrate and nitrite (Miller and Blair, 1971). The highest concentration of total nitrate (as N) was $18 \mathrm{mg} / \mathrm{L}$ at well (D-13-14)26bbb. The maximum total nitrite plus nitrate (as N) also was detected at this well and ranged from 15 to $25 \mathrm{mg} / \mathrm{L}$. The USEPA MCL of $10 \mathrm{mg} / \mathrm{L}$ for total nitrite plus nitrate (as $\mathrm{N}$ ) was exceeded for all three samples collected from well (D-13-14)26bbb. The most probable source of 
Table 3. Summary of selected physical and chemical data for ground-water sites, Rillito Creek basin, August 25, 1986, through March 28, 1989

$\left[\mathrm{mg} / \mathrm{L}\right.$, milligrams per liter, $\mu \mathrm{S} / \mathrm{cm}$, microsiemens per centimeter at $25^{\circ} \mathrm{C},{ }^{\circ} \mathrm{C}$; degrees Celsius; $\mathrm{NTU}$, nephelometetric-turbidity, $\mu \mathrm{g} / \mathrm{L}$, micrograms per liter; $\mathrm{pCi} / \mathrm{L}$, picocuries per liter]

\begin{tabular}{|c|c|c|c|c|}
\hline Constituent & $\begin{array}{l}\text { Number of } \\
\text { observations }\end{array}$ & Minimum & Maximum & Median \\
\hline \multicolumn{5}{|l|}{ Properties: } \\
\hline 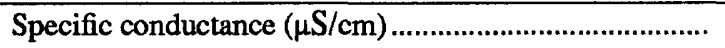 & 25 & 215 & 720 & 317 \\
\hline pH (units) & 25 & 6.7 & 7.9 & 7.3 \\
\hline Water temperature $\left({ }^{\circ} \mathrm{C}\right)$ & 25 & 17 & 25 & 20 \\
\hline Turbidity (NTU) & 25 & .1 & 90 & .4 \\
\hline Dissolved oxygen $(\mathrm{mg} / \mathrm{L})$ & 25 & 2.6 & 10.4 & 5.7 \\
\hline Hardness as $\mathrm{CaCO}_{3}(\mathrm{mg} / \mathrm{L})$ & 25 & 59 & 390 & 110 \\
\hline Alkalinity as $\mathrm{CaCO}_{3}(\mathrm{mg} / \mathrm{L})$ & 25 & 83 & 219 & 125 \\
\hline Dissolved solids at $180^{\circ} \mathrm{C}(\mathrm{mg} / \mathrm{L})$ & 25 & 132 & 689 & 202 \\
\hline \multicolumn{5}{|l|}{ Major ions: } \\
\hline Calcium, dissolved $(\mathrm{mg} / \mathrm{L})$ & 25 & 22 & 120 & 43 \\
\hline 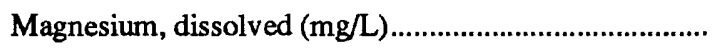 & 25 & 1 & 21 & 3.4 \\
\hline Sodium, dissolved $(\mathrm{mg} / \mathrm{L})$ & 25 & 7.2 & 55 & 25 \\
\hline Sodium adsorption ratio & 25 & .3 & 2 & .9 \\
\hline Potassium, dissolved $(\mathrm{mg} / \mathrm{L})$ & 25 & .9 & 3.1 & 1.9 \\
\hline Bicarbonate as $\mathrm{HCO}_{3}(\mathrm{mg} / \mathrm{L})$ & 25 & 101 & 267 & 152 \\
\hline Sulfate, dissolved $(\mathrm{mg} / \mathrm{L})$ & 25 & 10 & 42 & 20 \\
\hline Chloride, dissolved $(\mathrm{mg} / \mathrm{L})$ & 25 & 2 & 180 & 8.8 \\
\hline Fluoride, dissolved $(\mathrm{mg} / \mathrm{L})$ & 25 & .1 & .6 & .2 \\
\hline Silica, dissolved (mg/L) & 25 & 17 & 43 & 27 \\
\hline \multicolumn{5}{|l|}{ Nutrients $(\mathrm{mg} / \mathrm{L}):$} \\
\hline 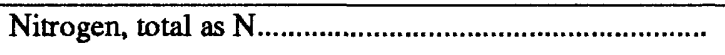 & 15 & .70 & 25 & 3.40 \\
\hline 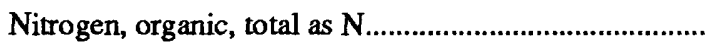 & 12 & .16 & 2.1 & .47 \\
\hline 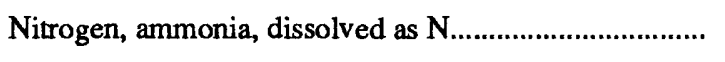 & 20 & $<.01$ & .05 & $<.01$ \\
\hline Nitrogen, ammonia, total as $\mathrm{N}$ & 20 & $<.01$ & .05 & .03 \\
\hline Nitrogen, ammonia plus organic, total as $\mathrm{N} . . . . . . . . . . . . . . . . . . . .$. & 20 & $<.20$ & 2.2 & .40 \\
\hline Nitrogen, nitrate, dissolved as $\mathrm{N}$ & 4 & .48 & 18 & .54 \\
\hline Nitrogen, nitrite, total as $\mathrm{N}$ & 20 & $<.01$ & .02 & .01 \\
\hline 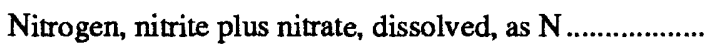 & 20 & .43 & 23 & 1.60 \\
\hline Nitrogen, nitrite plus nitrate, total as $\mathrm{N}$ & 20 & .45 & 25 & 1.75 \\
\hline Phosphate, total as $\mathrm{PO}_{4}$ & 12 & .03 & .18 & .08 \\
\hline 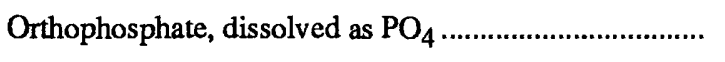 & 7 & .03 & .15 & .06 \\
\hline 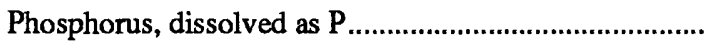 & 20 & $<.01$ & .06 & .01 \\
\hline Phosphorus, total as P & 20 & $<.01$ & .12 & .02 \\
\hline 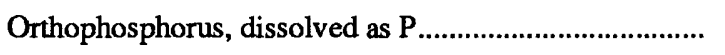 & 20 & $<.01$ & .05 & $<.01$ \\
\hline
\end{tabular}


Table 3. Summary of selected physical and chemical data for ground-water sites, Rillito Creek basin, August 25, 1986, through March 28, 1989-Continued

$\left[\mathrm{mg} / \mathrm{L}\right.$, milligrams per liter; $\mu \mathrm{S} / \mathrm{cm}$, microsiemens per centimeter at $25^{\circ} \mathrm{C},{ }^{\circ} \mathrm{C}$; degrees Celsius; NTU, nephelometetric-turbidity, $\mu \mathrm{g} / \mathrm{L}$, micrograms per liter; $\mathrm{pCi} / \mathrm{L}$, picocuries per liter]

\begin{tabular}{|c|c|c|c|c|}
\hline Constituent & $\begin{array}{c}\text { Number of } \\
\text { observations }\end{array}$ & Minimum & Maximum & Median \\
\hline \multicolumn{5}{|l|}{ Trace elements $(\mu \mathrm{g} / \mathrm{L})$ : } \\
\hline 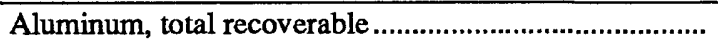 & 17 & $<10$ & 2,200 & 10 \\
\hline 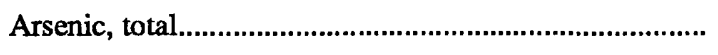 & 17 & $<1$ & 4 & 2 \\
\hline Barium, total recoverable & 17 & $<100$ & 400 & $<100$ \\
\hline 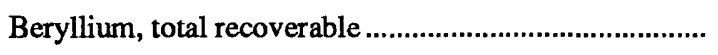 & 17 & $<10$ & $<10$ & $<10$ \\
\hline Cadmium, total recoverable & 17 & $<1$ & 1 & $<1$ \\
\hline 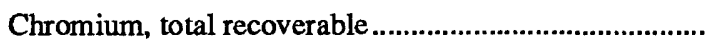 & 17 & $<10$ & 10 & $<10$ \\
\hline Cobalt, total recoverable & 17 & $<1$ & 3 & $<1$ \\
\hline Copper, total recoverable & 17 & 1 & 33 & 4 \\
\hline Iron, total recoverable & 17 & $<10$ & 3,400 & 160 \\
\hline Lead, total recoverable & 17 & $<5$ & 38 & $<5$ \\
\hline Lithium, total recoverable & 17 & $<10$ & 60 & 10 \\
\hline Manganese, total recoverable & 17 & $<10$ & 180 & 10 \\
\hline Mercury, total recoverable & 17 & $<.1$ & .1 & $<.1$ \\
\hline Molybdenum, total recoverable & 17 & $<1$ & 5 & 2 \\
\hline Nickel, total recoverable & 17 & $<1$ & 9 & 2 \\
\hline Selenium, total & 17 & $<1$ & 2 & $<1$ \\
\hline Silver, total recoverable & 17 & $<1$ & 1 & $<1$ \\
\hline Zinc, total recoverable & 17 & $<10$ & 100 & 30 \\
\hline \multicolumn{5}{|l|}{ Radionuclides: } \\
\hline 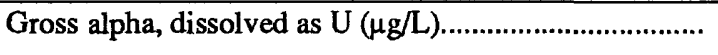 & 13 & $<.6$ & 9.2 & 3.3 \\
\hline Gross beta, dissolved as Sr-90/Y-90 (pCi $/ \mathrm{L}) \ldots \ldots \ldots \ldots \ldots \ldots \ldots$ & 13 & .6 & 4.6 & 2.2 \\
\hline Gross beta, dissolved as $\mathrm{Cs}-137(\mathrm{pCi} / \mathrm{L})$ & 13 & .7 & 6.7 & 2.9 \\
\hline Radon 222, total $(\mathrm{pCi} / \mathrm{L})$ & 13 & 120 & 690 & 400 \\
\hline \multicolumn{5}{|l|}{ Priority poiiutants, total recoverabie $(\mu \mathrm{g} / \mathrm{L})$} \\
\hline 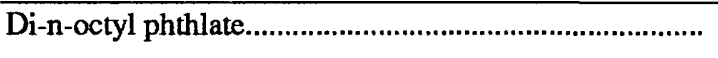 & 20 & $<10$ & 17 & $<10$ \\
\hline 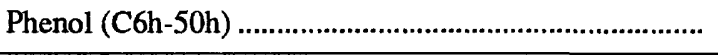 & 20 & $<5$ & 39 & $<5$ \\
\hline \multicolumn{5}{|l|}{ Volatile organic compounds $(\mu \mathrm{g} / \mathrm{L})$} \\
\hline 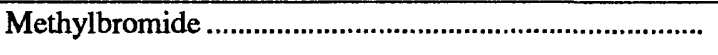 & 26 & $<3$ & 5.3 & $<3$ \\
\hline 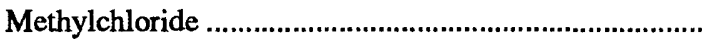 & 26 & .3 & 4.0 & $<3$ \\
\hline Toluene & 26 & $<3$ & 3.2 & $<3$ \\
\hline \multicolumn{5}{|l|}{ Organic carbon and oil and grease $(\mathrm{mg} / \mathrm{L})$ : } \\
\hline 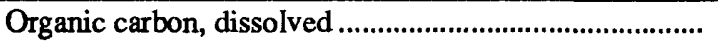 & 11 & .9 & 3.9 & 1.3 \\
\hline 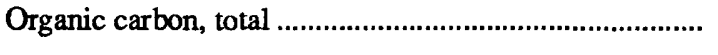 & 24 & .3 & 4.0 & 1.1 \\
\hline 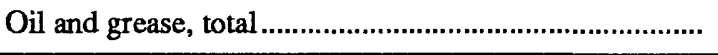 & 14 & $<1$ & $<1$ & $<1$ \\
\hline
\end{tabular}




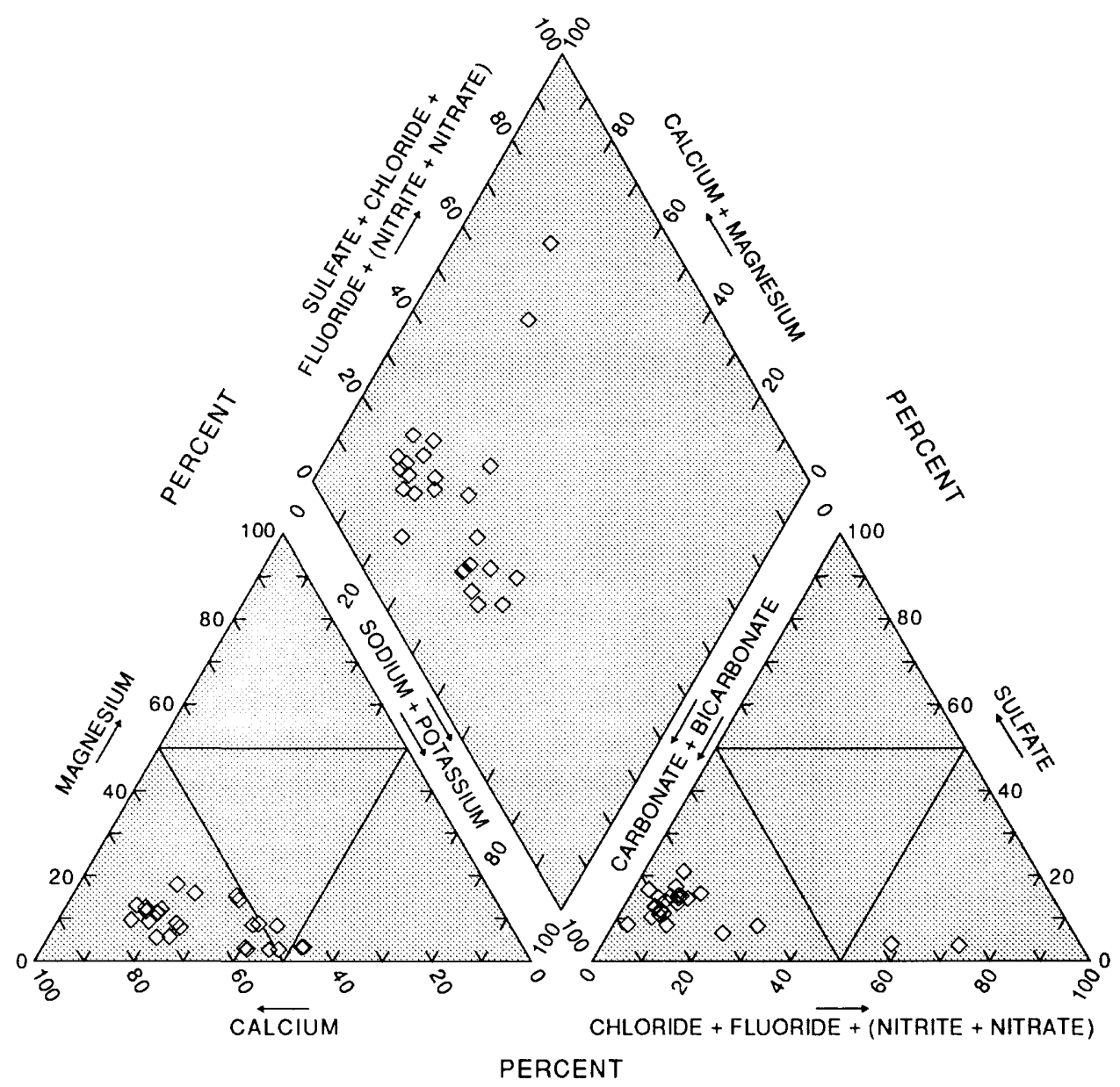

Figure 6. Relative compositions of ground water, in percent, Rillito Creek basin, 1986-89.

nitrate at this well is leachate from residential septic tanks in the area. Low concentrations of dissolved and total ammonia and total nitrite (as $\mathrm{N}$ ) were detected at all wells in the study area.

Concentrations of total phosphate $(0.18 \mathrm{mg} / \mathrm{L})$ and dissolved orthophosphate $(0.15 \mathrm{mg} / \mathrm{L})$ (as P) were highest at well (D-13-14)27bdb4. The highest concentration of total phosphorus (as P) of $0.12 \mathrm{mg} / \mathrm{L}$ was detected at well (D-13-14)26dcb2. Low concentrations of dissolved phosphorus and orthophosphorus (as P) were detected in ground water.

Trace elements.-The highest measured concentrations of total recoverable aluminum $(2,200 \mu \mathrm{g} / \mathrm{L})$, barium $(400 \mu \mathrm{g} / \mathrm{L})$, copper $(33 \mu \mathrm{g} / \mathrm{L})$, iron $(3,400 \mu \mathrm{g} / \mathrm{L})$, lead $(38 \mu \mathrm{g} / \mathrm{L})$, lithium
$(60 \mu \mathrm{g} / \mathrm{L})$, manganese $(180 \mu \mathrm{g} / \mathrm{L})$, and zinc $(100 \mu \mathrm{g} / \mathrm{L})$ were detected in ground-water samples. The highest lead concentrations, 11 and $38 \mu \mathrm{g} / \mathrm{L}$, were detected at wells (D-13-14)26dda2 and (D-13-14)34aaal, respectively. Maximum concentrations of total recoverable aluminum $(2,200 \mu \mathrm{g} / \mathrm{L})$ in one sample at well (D-13-14)26dcb2 and iron (380 to $3,400 \mu \mathrm{g} / \mathrm{L}$ ) in seven wells exceeded the USEPA MCL's of $50 \mu \mathrm{g} / \mathrm{L}$ and $300 \mu \mathrm{g} / \mathrm{L}$, respectively. The wells were not constructed for water-quality monitoring and construction materials could have contributed to increased concentrations of some metals such as copper, iron, lead, and zinc.

Radionuclides.-Radionuclides in ground water are derived naturally from contact with 
Table 4. Summary of selected particle-size distribution and selected chemical constituents for bottom-sediment sites, Rillito Creek basin, July 28, 1987, through February 20, 1992

$[\%$, percent; mm, millimeter, $\mathrm{mg} / \mathrm{kg}$, milligrams per kilogram; $\mu \mathrm{g} / \mathrm{g}$, micrograms per gram; $\mathrm{pCi} / \mathrm{g}$, picocuries per gram; $\mu \mathrm{g} / \mathrm{kg}$, micrograms per kilogram; g/kg, gram per kilogram; <, less than. DDD, dichlorodiphenyl-dichloroethane; DDE, dichlorodiphenylethylene; DDT, dichlorodiphenyltrichloroethane; PCB, polychlorinated biphenyl]

\begin{tabular}{|c|c|c|c|c|}
\hline Constituent & $\begin{array}{l}\text { Number of } \\
\text { observations }\end{array}$ & Minimum & Maximum & Median \\
\hline \multicolumn{5}{|l|}{ Particle-size distribution (\%): } \\
\hline Silt and clay $(<0.063 \mathrm{~mm})$ & 5 & 0.5 & 4.1 & 1 \\
\hline Sand $(0.062-2 \mathrm{~mm})$ & 5 & 86.8 & 99.5 & 99 \\
\hline \multicolumn{5}{|l|}{ Nutrients, total (mg/kg): } \\
\hline Nitrogen, ammonium as $\mathbf{N}$ & 9 & $<10$ & 15 & 1.7 \\
\hline 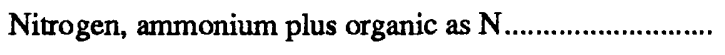 & 8 & 40 & 450 & 70 \\
\hline Nitrogen, nitrite plus nitrate as $\mathrm{N}$ & 9 & $<2$ & 26 & $<2$ \\
\hline Phosphorus as $\mathrm{P}$ & 9 & 160 & 240 & 190 \\
\hline \multicolumn{5}{|l|}{ Trace elements $(\mu \mathrm{g} / \mathrm{g})$ : } \\
\hline Aluminum, total recoverable & 9 & 54,000 & 76,300 & 65,000 \\
\hline Arsenic, total & 9 & $<10$ & 8 & $<10$ \\
\hline Barium, total recoverable & 9 & 660 & 1,000 & 750 \\
\hline Beryllium, total recoverable & 9 & 1 & 2 & 2 \\
\hline Cadmium, total recoverable & 9 & $<2$ & $<2$ & $<2$ \\
\hline Calcium, total recoverable & 9 & 8,400 & 56,000 & 22,000 \\
\hline Chromium, total recoverable & 9 & 3 & 51 & 30 \\
\hline Cobalt, total recoverable & 9 & 2 & 11 & 8 \\
\hline Copper, total recoverable & 9 & 4 & 83 & 30 \\
\hline Iron, total recoverable & 9 & 4,200 & 27,600 & 24,000 \\
\hline Lead, total recoverable & 9 & 20 & 58 & 30 \\
\hline 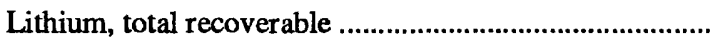 & 9 & 9 & 36 & 30 \\
\hline Magnesium, total recoverable & 9 & 800 & 9,500 & 7,000 \\
\hline Manganese, total recoverable & 9 & 180 & 1,180 & 520 \\
\hline Molybdenum, total recoverable & 9 & $<2$ & 2 & $<2$ \\
\hline Nickel, total recoverable & 9 & $<2$ & 20 & 10 \\
\hline Potassium, total recoverable & 9 & 21,000 & 32,000 & 26,000 \\
\hline Scandium, total recoverable & 9 & $<2$ & 80 & 8 \\
\hline Silver, total recoverable & 9 & $<2$ & $<2$ & $<2$ \\
\hline Sodium, total recoverable & 9 & 12,000 & 24,000 & 17,000 \\
\hline Strontium, total recoverable & 9 & 200 & 299 & 250 \\
\hline Tantalum, total recoverable & 9 & $<40$ & $<40$ & $<40$ \\
\hline Thorium, total recoverable & 9 & $<4$ & 16 & 10 \\
\hline Tin, total recoverable & 9 & $<5$ & 180 & $<5$ \\
\hline Titanium, total recoverable & 9 & 500 & 3,300 & 2,800 \\
\hline Uranium, total recoverable & 9 & $<100$ & $<100$ & $<100$ \\
\hline
\end{tabular}


Table 4. Summary of selected particle-size distribution and selected chemical constituents for bottom-sediment sites, Rillito Creek basin, July 28, 1987, through February 20, 1992-Continued

\begin{tabular}{|c|c|c|c|c|}
\hline Constituent & $\begin{array}{c}\text { Number of } \\
\text { observations }\end{array}$ & Minimum & Maximum & Median \\
\hline \multicolumn{5}{|l|}{ Trace elements $(\mu \mathrm{g} / \mathrm{g})-$ Continued: } \\
\hline 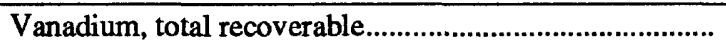 & 9 & 7 & 64 & 49 \\
\hline 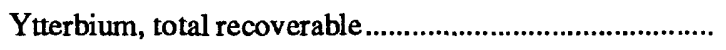 & 9 & $<1$ & 30 & 2 \\
\hline Zinc, total recoverable & 9 & 11 & 200 & 60 \\
\hline \multicolumn{5}{|l|}{ Radionuclides: } \\
\hline 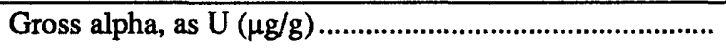 & 9 & 1 & 20.3 & 14 \\
\hline Gross alpha, as Th-230 $(\mu \mathrm{g} / \mathrm{g})$ & 8 & .7 & 17.1 & 10.5 \\
\hline 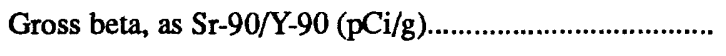 & 8 & 5.1 & 35 & 31.5 \\
\hline 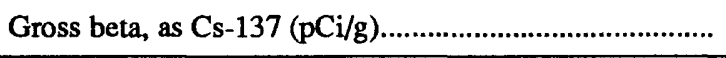 & 8 & 9.4 & 59 & 32 \\
\hline \multicolumn{5}{|l|}{ Organochlorine pesticides, total recoverabie $(\mu \mathrm{g} / \mathrm{kg})$} \\
\hline 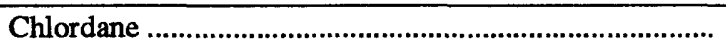 & 8 & $<10$ & 140 & $<10$ \\
\hline DDD & 8 & $<.1$ & .1 & $<.1$ \\
\hline DDE & 8 & $<.1$ & 3.4 & $<.1$ \\
\hline DDT & 8 & $<.1$ & 2.8 & $<.1$ \\
\hline 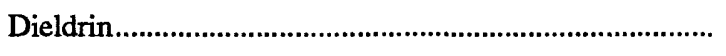 & 8 & $<.1$ & 10 & $<.1$ \\
\hline 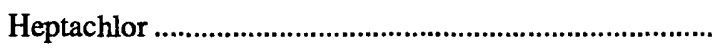 & 8 & $<.1$ & .9 & $<.1$ \\
\hline 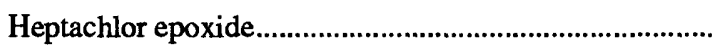 & 8 & $<.1$ & .12 & $<.1$ \\
\hline 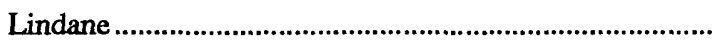 & 8 & $<.1$ & .2 & $<.1$ \\
\hline PCB & 8 & $<1.0$ & 13 & $<1.0$ \\
\hline 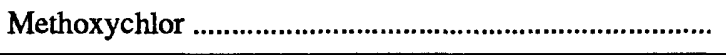 & 8 & $<.1$ & .5 & $<.1$ \\
\hline \multicolumn{5}{|l|}{ Priority poilutants, totai recoverable $(\mu \mathrm{g} / \mathrm{kg})$} \\
\hline Benzo $\mathrm{A}$ anthracene1,2-benzanthranene .............................. & 8 & $<400$ & 710 & $<400$ \\
\hline 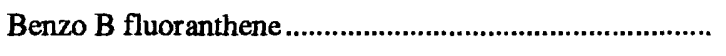 & 8 & $<400$ & 1,100 & $<400$ \\
\hline 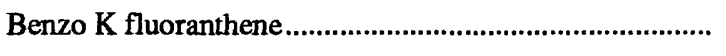 & 8 & $<400$ & 930 & $<400$ \\
\hline Benzo A pyrene & 8 & $<400$ & 850 & $<400$ \\
\hline Benzogh I perylene, 1, 2-benzoperylene ............................ & 8 & $<400$ & 760 & $<400$ \\
\hline 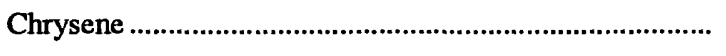 & 8 & $<400$ & 1,200 & $<400$ \\
\hline 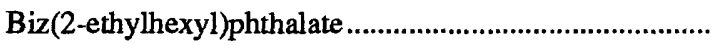 & 8 & $<200$ & 1,800 & $<200$ \\
\hline 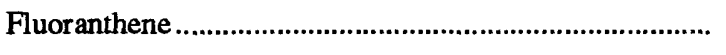 & 8 & $<200$ & 2,300 & $<200$ \\
\hline 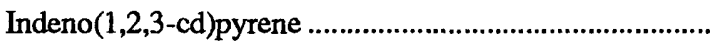 & 8 & $<400$ & 830 & $<400$ \\
\hline 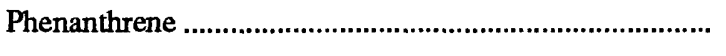 & 8 & $<200$ & 620 & $<200$ \\
\hline Pyrene & 8 & $<200$ & 2,000 & $<200$ \\
\hline \multicolumn{5}{|l|}{ Inorganjc carbon and organic carbon: } \\
\hline 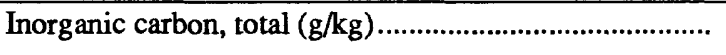 & 9 & .1 & 7.3 & 3.3 \\
\hline $\begin{array}{l}\text { Organic carbon plus inorganic } \\
\text { carbon, total }(\mathrm{g} / \mathrm{kg})\end{array}$ & 9 & 1 & 13 & 1.7 \\
\hline
\end{tabular}


Wash, 170 to $190 \mathrm{mg} / \mathrm{kg}$ at Alamo Wash, and 160 to $220 \mathrm{mg} / \mathrm{kg}$ at Rillito Creek.

Trace elements.-Total recoverable aluminum, barium, calcium, iron, magnesium, manganese, potassium, sodium, and titanium concentrations in bottom sediment were higher than concentrations of the other trace elements. Concentrations of trace elements in bottom sediment from all four sampling sites were not greatly different from each other (table 11). Cadmium, gold, silver, tantalum, and uranium concentrations were less than the detection limit, and molybdenum $(2 \mu \mathrm{g} / \mathrm{g})$ was detected in only one sample at Tanque Verde Creek.

Because of the absence of trace-element criteria for bottom sediment, analytical results from the sampling sites are compared with geochemical baseline information from soils of the western United States compiled by the U.S. Geological Survey (Shacklette and Boerngen, 1984). Table 5 has been modified from Shacklette and Boerngan to include only the constituent concentrations that were part of the chemical analyses. Soil-sample data in table 5 consists of selected natural soils west of the 97th meridian within the conterminous United States. Samples were collected at a depth of approximately 8 inches below land surface and at 50-mile intervals. The soil samples were oven dried and then sifted through a 2-millimeter sieve before analysis.

Comparison of bottom-sediment and soil data in table 5 indicates generally similar concentrations for most of the trace elements. With the exceptions of scandium and tin, the ranges of concentrations in bottom sediment were within the ranges found in soil and do not indicate a significant accumulation of trace elements.

Radionuclides. - The highest activity of gross alpha (as $U$ ) was detected in bottom sediment from Tanque Verde Creek ( $14 \mu \mathrm{g} / \mathrm{g})$, Pantano Wash $(16.6 \mu \mathrm{g} / \mathrm{g})$, Alamo Wash $(14 \mu \mathrm{g} / \mathrm{g})$, and Rillito Creek $(20.3 \mu \mathrm{g} / \mathrm{g})$. Activities of gross alpha (as Th-230) were detected at Alamo Wash and Rillito Creek and ranged from 10 to $17.1 \mathrm{pCi} / \mathrm{g}$ and 11 to $14.4 \mathrm{pCi} / \mathrm{g}$, respectively. Gross beta activity (as Sr-90/Y-90) was highest at Tanque Verde Creek ( $35 \mathrm{pCi} / \mathrm{g}$ ), and gross beta activity (as Cs-137) was highest at Alamo Wash (59 pCi/g).

Organochlorine pesticides.-Several organochlorine pesticides were detected in bottom- sediment samples collected at the four sites. Concentrations of these compounds, with the exception of chlordane, dieldrin, and PCB were slightly higher than the detection limit. The highest concentrations of organochlorine pesticides were found in bottom sediment from Alamo Wash. Concentrations of chlordane ranged from 1.0 to $31 \mu \mathrm{g} / \mathrm{kg}$ at Tanque Verde Creek, 1.0 to $140 \mu \mathrm{g} / \mathrm{kg}$ at Alamo Wash, and less than 0.1 to $1.0 \mu \mathrm{g} / \mathrm{kg}$ at Rillito Creek. Concentrations of DDE, heptachlor, heptachlor epoxide, and lindane were detected only in samples from Alamo Wash. Concentrations of DDT were detected in samples from Tanque Verde Creek and Alamo Wash and ranged from less than 0.1 to $1.3 \mu \mathrm{g} / \mathrm{kg}$ and 0.2 to $2.8 \mu \mathrm{g} / \mathrm{kg}$, respectively. Dieldrin concentrations ranged from less than 0.1 to $2.5 \mu \mathrm{g} / \mathrm{kg}$ at Tanque Verde Creek, 0.7 to $10 \mu \mathrm{g} / \mathrm{kg}$ at Alamo Wash, and less than 0.1 to $0.2 \mu \mathrm{g} / \mathrm{kg}$ at Rillito Creek. The highest concentrations of PCB were detected at Tanque Verde Creek $(5 \mu \mathrm{g} / \mathrm{kg})$ and at Alamo Wash (13 $\mu \mathrm{g} / \mathrm{kg})$. Methoxychlor was detected in one sample $(0.5 \mu \mathrm{g} / \mathrm{kg})$ from Pantano Wash.

Priority pollutants.-Eleven priority pollutants were detected in bottom-sediment samples collected at Alamo Wash. Fluoranthene and pyrene were the only priority pollutants detected at Tanque Verde Creek. The maximum concentrations of fluoranthene $(2,300 \mu \mathrm{g} / \mathrm{kg})$ and pyrene $(2,000 \mu \mathrm{g} / \mathrm{kg})$ were detected at Alamo Wash. Priority pollutants were detected in concentrations of approximately 2 to 12 times the detection limits.

Inorganic and organic carbon.- Inorganic and organic carbon were detected at all four sampling sites. Concentrations of inorganic carbon ranged from 0.1 to $2.1 \mathrm{~g} / \mathrm{kg}$ at Tanque Verde Creek, from 1.7 to $2.2 \mathrm{~g} / \mathrm{kg}$ at Pantano Wash, from 1.7 to $7.3 \mathrm{~g} / \mathrm{kg}$ at Alamo Wash, and 0.8 to $2.0 \mathrm{~g} / \mathrm{kg}$ at Rillito Creek. Inorganic carbon plus organic carbon ranged from 1 to $13 \mathrm{~g} / \mathrm{kg}$ at Tanque Verde Creek, 3.3 to $3.6 \mathrm{~g} / \mathrm{kg}$ at Pantano Wash, 2.2 to $9.0 \mathrm{~g} / \mathrm{kg}$ at Alamo Wash, and 1.4 to $3.1 \mathrm{~g} / \mathrm{kg}$ at Rillito Creek.

Oil and grease.-Oil and grease were not detected in any of the bottom-sediment samples. The detection limit for oil and grease in bottom sediment is $1,000 \mathrm{mg} / \mathrm{kg}$. 
Table 5. Trace-element concentrations in bottom sediment of the Rillito Creek basin and in soils of the western conterminous United States

[Minimum, maximum, median, and mean are reported in micrograms per gram $(\mu \mathrm{g} / \mathrm{g})$; mean is geometric; >, greater than; <, less than. Modified from Shacklette and Borengan (1984)]

\begin{tabular}{|c|c|c|c|c|c|c|}
\hline \multirow{2}{*}{ Constituent } & \multicolumn{3}{|c|}{ Bottom sediment } & \multicolumn{3}{|c|}{ Soll } \\
\hline & Minimum & Maximum & Median & Minimum & Maximum & Mean \\
\hline 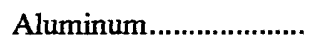 & 54,000 & 76,300 & 65,000 & 5,000 & $>100,000$ & 58,000 \\
\hline 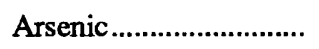 & $<10$ & 8 & $<10$ & $<.10$ & 97 & 5.5 \\
\hline Barium & 660 & 1,000 & 750 & 70 & 5,000 & 580 \\
\hline 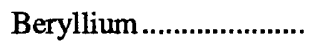 & 1 & 2 & 2 & $<1$ & 15 & .68 \\
\hline 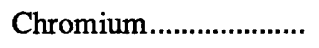 & 3 & 51 & 30 & 3 & 2,000 & 41 \\
\hline 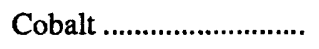 & 2 & 11 & 8 & $<3$ & 50 & 7.1 \\
\hline Copper & 4 & 83 & 30 & 2 & 300 & 21 \\
\hline Iron & 4,200 & 27,600 & 24,000 & 1,000 & 100,000 & 21,000 \\
\hline 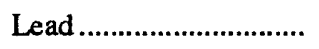 & 20 & 58 & 30 & $<10$ & 700 & 17 \\
\hline 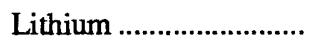 & 9 & 36 & 30 & 5 & 130 & 22 \\
\hline 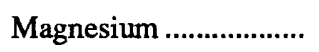 & 800 & 9,500 & 7,000 & 300 & $>100,000$ & 7,400 \\
\hline 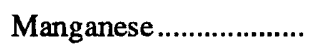 & 180 & 1,180 & 520 & 30 & 5,000 & 380 \\
\hline 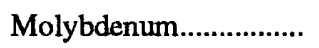 & $<2$ & 2 & $<2$ & $<3$ & 7 & .85 \\
\hline 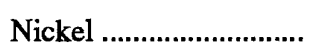 & $<2$ & 20 & 10 & $<5$ & 700 & 15 \\
\hline 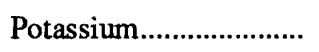 & 21,000 & 32,000 & 26,000 & 1,900 & 630,000 & 18,000 \\
\hline Scandium & $<2$ & 80 & 8 & $<5$ & 50 & 8.2 \\
\hline Sodium & 12,000 & 24,000 & 17,000 & 500 & 100,000 & 9,700 \\
\hline 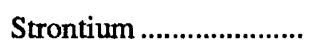 & 200 & 299 & 250 & 10 & 3,000 & 200 \\
\hline 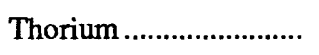 & $<4$ & 16 & 10 & 2.4 & 31 & 9.1 \\
\hline Tin & $<5$ & 180 & $<5$ & $<0.1$ & 7.4 & .9 \\
\hline 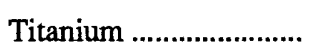 & 500 & 3,300 & 2,800 & 500 & 20,000 & 2,200 \\
\hline 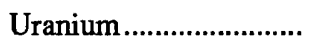 & $<100$ & $<100$ & $<100$ & .68 & 7.9 & 2.5 \\
\hline 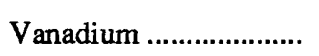 & 7 & 64 & 49 & 7 & 500 & 70 \\
\hline 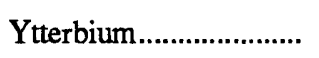 & $<1$ & 30 & 2 & $<10$ & 150 & 22 \\
\hline Zinc.................................... & 11 & 200 & 60 & 10 & 2,100 & 55 \\
\hline
\end{tabular}




\section{Comparison of Surface-Water and Ground-Water Quality}

According to Hem (1989), the chemical composition of natural water is derived from many different sources of solutes, including gases and aerosols from the atmosphere, weathering and erosion of rocks and soil, solution or precipitation reactions occurring below the land surface, and human activities. The chemical composition of ground water in the study area is affected by streamflow recharge, underflow, geology, mineralogy, internal and external drainage patterns, and historical development (Anderson and others, 1992). Recharge water infiltrating the unsaturated zone may undergo many physical, chemical, and biological processes (Crites and Nolte, 1985; Knorr and Client, 1985; Mackay and others, 1985; Oaksford, 1985). Such processes may include dissolution; ion exchange; adsorption; filtration; precipitation; volatilization; and physical, chemical, and microbial degradation (Miller and Blair, 1971; DeCook and Wilson, 1980; Mooradian, 1983; Olson, 1987). Filtration and adsorption are the most important purification processes, playing a vital part in quality improvement and in the attenuation of constituents in the unsaturated zone during infiltration (Miller and Blair, 1971; Crites, 1985; Huisman and Olsthhoorn, 1983; Miller, 1990). Median values for selected physical and chemical data in surface water and ground water are presented in table 6.

Properties.- $\mathrm{pH}$ ranged from 6.5 to 9.2 with a median value of 8.3 in surface water and ranged from 6.7 to 7.9 with a median value of 7.3 in ground water. Specific conductance, hardness, and dissolved-solids concentrations were greater in ground water than in surface water. Median values for hardness indicate that surface water is soft and ground water is moderately hard. Concentrations of dissolved solids ranged from 39 to $243 \mathrm{mg} / \mathrm{L}$ in surface water and 132 to $689 \mathrm{mg} / \mathrm{L}$ in ground water.

Major ions.-Concentrations of all major ions, with the exception of potassium, were greater in ground water than in surface water. The largest differences were in concentrations of bicarbonate, calcium, sodium, sulfate, and silica. According to Hem (1989), concentrations of dissolved silica commonly are considerably higher in ground water than in surface water because silica is a constituent of most igneous rocks and are found in some form in most other rocks and soils. Concentrations of major ions generally were lower in ground water along the Rillito Creek than in ground water in the surrounding areas. The surface water is a calcium and bicarbonate type and the ground water is a calcium sodium and bicarbonate type.

Nutrients.-Concentrations of total organic nitrogen (as $\mathrm{N}$ ) and ammonia (as $\mathrm{N}$ ) were higher in surface water than in ground water. Concentrations of dissolved and total nitrite plus nitrate (as $N$ ) were higher in ground water than in surface water.

Concentrations of total phosphate (as $\mathrm{PO}_{4}$ ), dissolved orthophosphate (as $\mathrm{PO}_{4}$ ), dissolved and total phosphorus (as P), and dissolved orthophosphorus (as P) were higher in surface water than in ground water. According to Bouwer (1989), most of the phosphates in ground water probably are removed by precipitation of calcium phosphate.

Trace elements.-Median values for selected trace elements in surface water and ground water are presented in table 6 . The median values for total recoverable trace elements, with the exception of molybdenum, in surface water are higher than in ground water.

Radionuclides.-The median values for dissolved activities of gross beta (as Sr-90/Y-90) and dissolved gross beta (as Cs-137) were lower in ground water than in surface water. Dissolved gross alpha activity (as U) was higher in ground water.

Organochlorine pesticides.-Several organochlorine pesticides were detected in surfacewater and bottom-sediment samples. Chlordane was found at higher concentrations than other pesticides. Some of the pesticides in surface water and bottom sediment probably were caused by use of chemicals to control weeds and insects in urban and agricultural areas. None of the organochlorine pesticides were detected in the ground-water samples.

Priority pollutants.-Several priority pollutants were detected in surface-water, ground-water, and bottom-sediment samples. None of the priority pollutants that were detected in ground-water samples were detected in surface-water or bottom-sediment samples.

Volatile organic compounds.-Volatile organic compounds were not detected in surfacewater samples. Low concentrations of three VOC's 
Table 6. Median values of physical and chemical data for surface-water and ground-water samples, Rillito Creek basin, August 25, 1986, through February 13, 1992

$\left[\mu \mathrm{S} / \mathrm{cm}\right.$, microsiemens per centimeter at $25^{\circ} \mathrm{C},{ }^{\circ} \mathrm{C}$, degrees Celsius; NTU, nephelometric-turbidity units; mg/L, milligrams per liter, $\mu \mathrm{g} / \mathrm{L}$, micrograms per liter, $\mathrm{pCi} / \mathrm{L}$, picocuries per liter]

\begin{tabular}{|c|c|c|c|c|c|}
\hline Constituent & $\begin{array}{c}\text { Surface } \\
\text { water }\end{array}$ & $\begin{array}{l}\text { Ground } \\
\text { water }\end{array}$ & Constltuent & $\begin{array}{c}\text { Surface } \\
\text { water }\end{array}$ & $\begin{array}{l}\text { Ground } \\
\text { water }\end{array}$ \\
\hline Properties: & & & Nutrients $(\mathrm{mg} / \mathrm{L})-$ Continued: & & \\
\hline Specific conductance $(\mu \mathrm{S} / \mathrm{cm}) .$. & 79 & 317 & Phosphorus, total as P................ & 0.42 & 0.02 \\
\hline 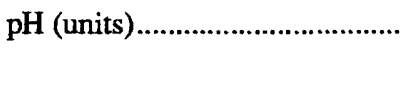 & 8.3 & 7.3 & 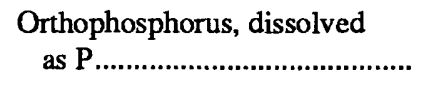 & .08 & $<.01$ \\
\hline Dissolved oxygen $(\mathrm{mg} / \mathrm{L}) \ldots \ldots \ldots$ & 6.6 & 5.7 & Trace elements $(\mu \mathrm{g} / \mathrm{L}):$ & & \\
\hline Hardness as $\mathrm{CaCO}_{3}(\mathrm{mg} / \mathrm{L}) \ldots . .$. & 35 & 110 & Aluminum, total recoverable .... & 89,500 & 10 \\
\hline Alkalinity as $\mathrm{CaCO}_{3}(\mathrm{mg} / \mathrm{L}) \ldots$ & 31 & 125 & 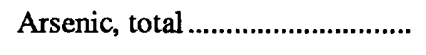 & 8 & 2 \\
\hline $\begin{array}{l}\text { Dissolved solids at } 180^{\circ} \mathrm{C} \\
\quad(\mathrm{mg} / \mathrm{L})\end{array}$ & 85 & 202 & Barium, total recoverable............ & 1,000 & $<100$ \\
\hline Major ions: & & & Beryllium, total recoverable ..... & $<10$ & $<10$ \\
\hline Calcium, dissolved $(\mathrm{mg} / \mathrm{L}) . . . \ldots .$. & 11 & 43 & Cadmium, total recoverable...... & 2 & $<1$ \\
\hline Magnesium, dissolved (mg/L).. & 1.3 & 3.4 & Chromium, total recoverable .... & 84 & $<10$ \\
\hline Sodium, dissolved $(\mathrm{mg} / \mathrm{L})$......... & 4.5 & 25 & Cobalt, total recoverable ............. & 30 & $<1$ \\
\hline Sodium adsorption ratio ............ & .4 & .9 & Copper, total recoverable ........... & 260 & 4 \\
\hline Potasium, dissolved (mg/L)...... & 2.1 & 1.9 & Iron, total recoverable ................. & 85,000 & 160 \\
\hline Bicarbonate as $\mathrm{HCO}_{3}(\mathrm{mg} / \mathrm{L}) \ldots$ & 37 & 152 & Lead, total recoverable................ & 155 & $<5$ \\
\hline Sulfate, dissolved $(\mathrm{mg} / \mathrm{L})$.......... & 7.2 & 20 & Lithium, total recoverable.......... & 110 & 10 \\
\hline Chloride, dissolved $(\mathrm{mg} / \mathrm{L}) . . . . .$. & 3.0 & 8.8 & Manganese, total recoverable.... & 4,900 & 10 \\
\hline Fluoride, dissolved (mg/L) ........ & .1 & .2 & Mercury, total recoverable ........ & .3 & $<.1$ \\
\hline Silica, dissolved $(\mathrm{mg} / \mathrm{L}) \ldots \ldots \ldots \ldots$ & 8.2 & 27 & Molybdenum, total recoverable & 1 & 2 \\
\hline Nutrients (mg/L): & & & Nickel, total recoverable............ & 97 & 2 \\
\hline Nitrogen, total as N..................... & 1.40 & 3.40 & Selenium, total ................................ & $<1$ & $<1$ \\
\hline Nitrogen, organic, total as $\mathbf{N} . . .$. & .80 & .47 & Silver, total recoverable ............. & $<1$ & $<1$ \\
\hline 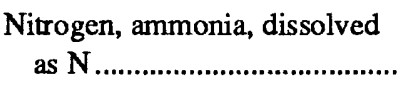 & .06 & $<.01$ & Zinc, total recoverable ................ & 790 & 30 \\
\hline Nitrogen, ammonia, total as N.. & .08 & .03 & Radionuclides: & & \\
\hline $\begin{array}{l}\text { Nitrogen, ammonia plus } \\
\quad \text { organic, total as } \mathrm{N} . . . . . . . . . . . . . . . .\end{array}$ & 1.00 & .40 & 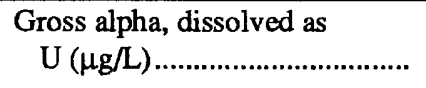 & .8 & 3.3 \\
\hline 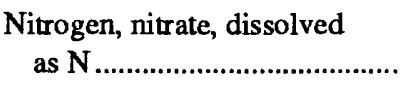 & .22 & .54 & 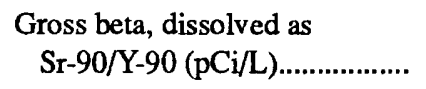 & 3.1 & 2.2 \\
\hline Nitrogen, nitrite, total as $\mathrm{N} . . . . .$. & .02 & .01 & 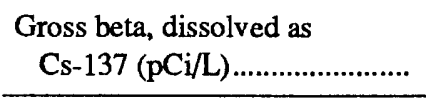 & 4.1 & 2.9 \\
\hline $\begin{array}{l}\text { Nitrogen, nitrite plus nitrate, } \\
\text { dissolved, as } N \text {............................ }\end{array}$ & .27 & 1.60 & Organic carbon, $(\mathrm{mg} / \mathrm{L})$ : & & \\
\hline $\begin{array}{l}\text { Nitrogen, nitrite plus nitrate, } \\
\text { total as } \mathrm{N} . . . . . . . . . . . . . . . . . . . . . . . . . . . . . .\end{array}$ & .32 & 1.75 & Organic carbon, dissolved.......... & 11.5 & 1.3 \\
\hline 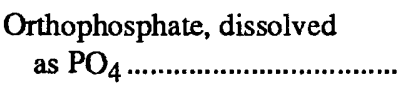 & .42 & .06 & Organic carbon, total................... & 50.5 & 1.1 \\
\hline Phosphorus, dissolved as P....... & .09 & .01 & & & \\
\hline
\end{tabular}


were detected in five ground-water samples but were not detected in subsequent resampling.

Organic carbon--Concentrations of dissolved and total organic carbon were higher in surface water than in ground water. Lower concentrations of organic carbon in ground water could have resulted from adsorption.

Oil and grease.-Low concentrations of oil and grease were detected in 7 of 25 surface-water samples. Oil and grease were not detected in any of the ground-water and bottom-sediment samples.

\section{SUMMARY}

Controlled artificial recharge of surface runoff is being considered as a water-management technique to address the problem of ground-water overdraft. The use of recharge facilities in urban areas has caused concern about the quality of urban runoff to be recharged and the potential for ground-water contamination. The proposed ground-water recharge in Rillito Creek will utilize runoff entering a 1-mile reach of the creek between Craycroft and Swan Roads for infiltration and recharge purposes within the channel and excavated overbank areas.

Physical and chemical data were collected from 4 surface-water, 14 ground-water, and 4 bottom-sediment sites during 1986-92. Surfacewater and bottom-sediment samples were collected in order to determine the occurrence and concentrations of contaminants. Ground water from nearby wells was sampled and analyzed to determine general water-quality conditions in the recharge area.

The response of ground-water levels to streamflow in Tanque Verde Creek is shown by hydrographs from wells and the gaging-station data. Discharge was determined at the four sites from stage readings using rating curves developed from previous current-meter measurements.

Sediment concentrations tended to increase in relation to an increase in streamflow and were higher during the summer. Suspended-sediment concentrations ranged from 22 to $36,700 \mathrm{mg} / \mathrm{L}$ in surface water.

The median value of $\mathrm{pH}$ was higher in surface water than in ground water. Specific conductance, hardness, and dissolved-solids concentrations generally were greater in ground water than in surface water. Dissolved-solids concentrations ranged from 39 to $243 \mathrm{mg} / \mathrm{L}$ in surface water and 132 to $689 \mathrm{mg} / \mathrm{L}$ in ground water. The dissolved-solids concentration of $689 \mathrm{mg} / \mathrm{L}$ in one sample at well (D-13-14)26bbb exceeded the SMCL of $500 \mathrm{mg} / \mathrm{L}$.

The concentrations of all major ions analyzed, with the exception of potassium, were greater in ground water than in surface water. The largest differences were in bicarbonate, calcium, sodium, sulfate, and silica concentrations. The surface water is a calcium and bicarbonate type and the ground water is a calcium sodium and bicarbonate type.

Total organic nitrogen (as $\mathrm{N}$ ) was highest in surface water and ranged from 0.17 to $9.7 \mathrm{mg} / \mathrm{L}$. The maximum concentration of total nitrite plus nitrate (as $\mathrm{N}$ ) in ground water ranged from 15 to $25 \mathrm{mg} / \mathrm{L}$ and exceeded the MCL of $10 \mathrm{mg} / \mathrm{L}$. The most probable source of nitrate in ground water is leachate from residential septic tanks in the area.

Concentrations of total and dissolved phosphate and orthophosphates (as P) were higher in surface water than in ground water. Most of the phosphate in ground water probably is removed by precipitation of calcium phosphate.

The highest concentrations of total recoverable barium $(10,000 \mu \mathrm{g} / \mathrm{L})$, cadmium $(12 \mu \mathrm{g} / \mathrm{L})$, chromium $(350 \mu \mathrm{g} / \mathrm{L})$, lead $(1,900 \mu \mathrm{g} / \mathrm{L})$ and mercury $(2.8 \mu \mathrm{g} / \mathrm{L})$ were detected in surface water and exceeded the State of Arizona MCL's for drinking water.

The highest concentrations of total recoverable lead $(11$ and $38 \mu \mathrm{g} / \mathrm{L})$ were detected at wells (D-13-14)26dda2 and (D-13-14)34aaa1, respectively. Maximum concentrations of total recoverable aluminum (60 to $2,200 \mu \mathrm{g} / \mathrm{L}$ ) in five wells and iron ( 380 to $3,400 \mu \mathrm{g} / \mathrm{L}$ ) in seven wells exceeded the USEPA SMCL's of 50 and $300 \mu \mathrm{g} / \mathrm{L}$, respectively.

Analyses of bottom sediments for trace elements were compared with baseline geochemical information for soils of the western conterminous United States compiled by the U.S. Geological Survey. Concentrations of trace elements in bottom sediment generally were similar to reported concentrations in soils of the western conterminous United States and do not suggest a significant accumulation of these elements. 
The unadjusted median value of $93 \mu \mathrm{g} / \mathrm{L}$ for suspended gross alpha activity (as $U$ ) that converts to $63.2 \mathrm{pCi} / \mathrm{L}$ (assuming a 0.68 conversion factor) could exceed the State of Arizona MCL for drinking water of $15 \mathrm{pCi} / \mathrm{L}$ for adjusted gross alpha (minus uranium and radon). None of the dissolved gross alpha activities (as U) exceeded the USEPA MCL for drinking water. Dissolved gross alpha and dissolved gross beta were significantly lower than suspended gross alpha and suspended gross beta because the suspended material larger than 0.45 -micrometer was removed by filtration. In ground-water samples, the median value of 400 $\mathrm{pCi} / \mathrm{L}$ for radon exceeded the USEPA proposed MCL of $300 \mathrm{pCi} / \mathrm{L}$.

Seven organochlorine pesticides were detected in surface-water samples and ten were detected in bottom-sediment samples. Chlordane ranged from less than 0.1 to $150 \mu \mathrm{g} / \mathrm{L}$ and less than 10 to $140 \mathrm{mg} / \mathrm{kg}$, in surface water and bottom sediment, respectively. The presence of some of the pesticides in surface water and bottom sediment is most likely the result of frequent use of the chemicals to control weeds and insects in urban and agricultural areas. None of the organochlorine pesticides were detected in ground water.

Three priority pollutants were detected in surface-water samples, two were detected in ground-water samples, and eleven were detected in bottom-sediment samples. Laboratory or field contamination most likely were responsible for detection of di-n-octyl phthalate in ground-water samples. The presence of the other priority pollutants in surface water and bottom sediment is attributed to a higher intensity of urbanization and the type of land use within the Alamo Wash and Rillito Creek watersheds.

Volatile organic compounds were not detected in samples from surface water. Low concentrations of methylbromide, methylchloride, and toluene were detected in five samples of ground water but were not detected in subsequent sampling. None of the constituents were detected in the resampling of these wells.

Concentrations of dissolved and total organic carbon were higher in surface water than in ground water. Adsorption can reduce the amount of organics in ground water.

Low concentrations of oil and grease were detected in 7 of 25 surface-water samples. Oil and grease were not detected in any of the ground-water and bottom-sediment samples. The presence of oil and grease in surface water was most likely the result of rainfall runoff from roads and parking lots.

\section{SELECTED REFERENCES}

Anderson, S.R., 1987, Cenozoic stratigraphy and geologic history of the Tucson basin, Pima County, Arizona: U.S. Geological Survey Water-Resources Investigations Report 87-4190, $20 \mathrm{p}$.

1988, Potential for aquifer compaction, land subsidence, and earth fissures in the Tucson basin, Pima County, Arizona: U.S. Geological Survey Hydrologic Investigations Atlas HA-713, 3 sheets.

Anderson, T.W., Freethey, G.W., and Tucci, Patrick, 1992, Geohydrology and water resources of alluvial basins in south-central Arizona and parts of adjacent States: U.S. Geological Survey Professional Paper 1406-B, $60 \mathrm{p}$.

Ayers, R.S., and Westcot, D.W., 1985, Water quality for agriculture: Rome, Italy, United Nations, Food and Agriculture Organization Irrigation and Drainage Paper 29, rev. 1, $174 \mathrm{p}$.

Bouwer, Herman, 1989, Groundwater recharge with sewage effluent, in Johnson, A.I. and Finlayson, D.J., eds., Artificial Recharge of Ground Water: New York, American Society of Civil Engineers, Anaheim, California, August 23-27, 1988, Proceedings, p. 170-185.

Brown, R.F., and Signor, D.C., 1974, Artificial recharge-State of art: Ground Water, v. 12, no. 3, p. $152-160$.

Burkham, D.E., 1970, Depletion of streamflow by infiltration in the main channels of the Tucson basin, southern Arizona: U.S. Geological Survey Water-Supply Paper 1939-B, 36 p.

Camp Dresser and McKee, Inc., 1990, Rillito Creek recharge feasibility study, water sources, hydrogeology and water quality: Camp Dresser and McKee, Inc., Rillito Project Management Committee, Phase A, Tasks 1, 2, 3, and 4, v.p.

City of Tucson, 1987-93, Annual static water level-Basic data report, Tucson basin and Avra Valley, and Pima County, Arizona: Tucson, Arizona, City of Tucson, Water Department annual report.

Condes de la Torre, Alberto, 1970, Streamflow in the upper Santa Cruz River basin, Santa Cruz and Pima 
Counties, Arizona: U.S. Geological Survey Water-Supply Paper 1939-A, 26 p.

Crites, R.W., 1985, Micropollutant removal in rapid infiltration, in Asano, Takashi, ed., Artificial recharge of ground water: Boston, Massachusetts, Butterworth Publishers, p. 579-609.

Dharmadhikari, V.V., 1970, Quality of runoff from diversified urban watersheds: Tucson, Arizona, University of Arizona, master's thesis, $107 \mathrm{p}$.

Davidson, E.S., 1973, Geohydrology and water resources of the Tucson basin, Arizona: U.S. Geological Survey Water-Supply Paper 1939-E, $81 \mathrm{p}$.

DeCook, K.J., and Waterstone, Marvin, 1987, Central Arizona Project Water Quality-An examination of management options: Water Resources Research Center, University of Arizona, 100 p.

DeCook, K.J, and Wilson, L.G, 1980, Ground-water recharge from urban runoff and irrigation returns, in Proceedings of the Deep Percolation Symposium: Phoenix, Arizona Department of Water Resources Report No. 1, Scottsdale, Arizona, May 1-2, 1980, p. 37-52.

Dunn, I.S., Anderson, L.R., and Kiefer, F.W., 1980, Fundamentals of geotechnical analysis: New York, John Wiley and Sons, $414 \mathrm{p}$.

Freethey, G.W., and Anderson, T.W., 1986, Predevelopment hydrologic conditions in the alluvial basins of Arizona and adjacent parts of California and New Mexico: U.S. Geological Survey Hydrologic Investigations Atlas HA-664, scale $1: 500,000,3$ sheets.

Freethey, G.W., Pool, D.R., Anderson, T.W., and Tucci, Patrick, 1986, Generalized distribution of aquifer lithology in the alluvial basins of Arizona and adjacent parts of California and New Mexico: U.S. Geological Survey Hydrologic Investigations Atlas HA-663, scale 1:500,000, 4 sheets.

Guy, H.P., and Norman, V.W., 1970, Field methods for measurement of fluvial sediment: U.S. Geological Survey Techniques of Water-Resources Investigations, book 3, chap. C2, 59 p.

Hem, J.D., 1989, Study and interpretation of the chemical characteristics of natural water: U.S. Geological Survey Water-Supply Paper 2254, $263 \mathrm{p}$.

Hillel, Daniel, 1980, Fundamentals of soil physics: New York, Academic Press, Inc., 413 p.

Horowitz, A.J., 1985, A primer on trace metal-sediment chemistry: U.S. Geological Survey Water-Supply Paper 2277, 67 p.
Huisman, L., and Olsthhoorn, T.N., 1983, Artificial groundwater recharge: Boston, Massachusetts, Pitman Advanced Publishing Program, 320 p.

Kaddour, B.H., 1983, Recharge from ephemeral streams - case study in Arizona: Tucson, Arizona, University of Arizona, master's thesis, $120 \mathrm{p}$.

Katz, L.T., 1987, Steady-state infiltration processes along the Santa Cruz and Rillito Rivers: Tucson, Arizona, University of Arizona, master's thesis, $119 \mathrm{p}$.

Keith, S.J.S., 1981, Stream channel recharge in the Tucson basin and its implications for ground-water management: Tucson, Arizona, University of Arizona, master's thesis, $84 \mathrm{p}$.

Knorr, D.B., and Cliett, Tom, 1985, Proposed groundwater recharge at El Paso, Texas, in Asano, Takashi, ed., Artificial Recharge of Groundwater: Boston, Butterworth Publishers, p. 425-480.

Laney, R.L., 1972, Chemical quality of the water in the Tucson basin, Arizona: U.S. Geological Survey Water-Supply Paper 1939-D, 46 p.

MacKay, D.M., Roberts, P.V., and Cherry, J.A., 1985, Transport of organic contaminants in groundwater: Environmental Science Technology, v. 19, no. 5, p. 384-392.

Marron, Hal, Phinney, Dennis, and Musgrove, Judy, 1989, Artificial recharge and well rehabilitation for management of ground-water nitrates in Peoria, Arizona, in Fourth symposium on artificial recharge of ground water in Arizona: Phoenix, Arizona, Salt River Project, Proceedings, May 23-24, 1989, p. 105-111.

Matlock, W.G., 1965, The effect of silt-laden water on infiltration in alluvial channels: Tucson, Arizona, University of Arizona, doctoral dissertation, $102 \mathrm{p}$.

Miller, C.J., 1990, Impact of artificial recharge on Tucson area ground-water quality: Tucson, Arizona, University of Arizona, master's thesis, $152 \mathrm{p}$.

Miller, D.G., and Blair, A.H., 1971, The principles and practice of pretreatment for artificial recharge, in Artificial Groundwater Recharge: Buckinghamshire, England, The Water Research Association Proceedings, University of Reading, England, September 21-24, 1970, v. 1, p. 83-109.

Mooradian, M.M., 1980, The impact of urban runoff, Assessment of the potential for ground-water pollution as a result of urban runoff in the Tucson area: Pima Association of Governments (project 208), $35 \mathrm{p}$. 
1983, The Ina Road landfill as a source of ground-water pollution: Tucson, Arizona, University of Arizona, master's thesis, $86 \mathrm{p}$.

Moore, J.W., 1991, Inorganic contaminants of surface water-Research and monitoring priorities: New York, Springer-Verlag, $334 \mathrm{p}$.

Oaksford, E.T., 1985, Artificial recharge-Methods, hydraulics, and monitoring, in Asano, Takashi, ed., Artificial recharge of groundwater: Boston, Massachusetts, Butterworth Publishers, p. 69-127.

Olson, K.L., 1987, Urban stormwater injection via dry wells in Tucson, Arizona, and its effect on ground-water quality: Tucson, Arizona, University of Arizona, master's thesis, $151 \mathrm{p}$.

Olson, M.C., 1982, Mountain-front recharge to the Tucson basin from Tanque Verde Canyon, Arizona: Tucson, Arizona, University of Arizona, master's thesis, $145 \mathrm{p}$.

Pashley, E.F., 1966, Structure and stratigraphy of the central, northern, and eastern parts of the Tucson basin, Arizona: U.S. Geological Survey open-file report, $273 \mathrm{p}$.

Richter, R.C., and Chun, R.Y.D, 1961, Artificial recharge in California: American Society of Civil Engineers Transactions, v. 126, p. 742-766.

Robertson, F.N., 1991, Geochemistry of ground water in alluvial basins of Arizona and adjacent parts of Nevada, New Mexico, and California: U.S. Geological Survey Professional Paper 1406-C, $90 \mathrm{p}$.

Sayre, W.W., Guy, H.P., and Chamberlain, A.R., 1963, Uptake and transport of radionuclides by stream sediments: U.S. Geological Survey Professional Paper 433-A, $35 \mathrm{p}$.

Shacklette, H.T., and Boerngen, J.G., 1984, Element concentrations in soils and other surficial materials of the conterminous United States: U.S. Geological Survey Professional Paper 1270, 105 p.

Schmidt, K.D., Esposito, D.M., and Eaker, D.G., 1989, Contaminant hydrology associated with river recharge of sewage effluent, in Fourth Symposium on Artificial Recharge of Ground Water in Arizona: Phoenix, Arizona, Salt River Project Proceedings, May 23-24, 1989, p. 1-20.

State of Arizona, 1991, Drinking water and certification: Phoenix, Arizona, State of Arizona, Arizona Administrative Code, Title 18, Chapter 4, R-18-4-241 through 245, p. 22-35.
Thurman, E.M., 1985, Organic geochemistry of natural waters: Dordrecht, The Netherlands, Martinus Nijhoff and Dr. W. Junk Publishers, 494 p.

U.S. Environmental Protection Agency, 1993a, National revised drinking water regulations-Maximum contaminant levels: Washington D.C., U.S. Environmental Protection Agency, U.S. Code of Federal Regulations, Title 40, Part 141, July 1, 1993, p. 592-732.

1993b, National revised drinking water regulations-Secondary maximum contaminant levels: Washington D.C., U.S. Environmental Protection Agency, U.S. Code of Federal Regulations, Title 40, Part 143, July 1, 1993, p. 774-777.

U.S. Geological Survey, 1976, Water Resources Data for Arizona, water year 1975: U.S. Geological Survey Water-Data Report AZ-75-1, 404 p.

1977, National handbook of recommended methods for water-data acquisition-Chapter 5, Chemical and physical quality of water and sediment: Washington, D.C., U.S. Geological Survey, Office of Water-Data Coordination, $193 \mathrm{p}$. 1985, Water quality laboratory services catalog: U.S. Geological Survey Open-File Report 85-171, 5 parts, v.p.

Ward, J.R., and Harr, C.A., eds., 1990, Methods for collection and processing of surface-water and bed-material samples for physical and chemical analysis: U.S. Geological Survey Open-File Report 90-140, $71 \mathrm{p}$.

Wilson, L.G., 1985, Overview of artificial recharge methods, in Marsh, F.L., ed., Second Symposium on Artificial Recharge in Arizona: Tucson, University of Arizona Water Resources Center, May 2-3, 1984, p. 19-35.

Wilson, L.G., DeCook, K.J., and Neuman, S.P., 1980, Regional recharge research for southwest alluvial basins: University of Arizona, Water Resources Research Center, Final Report for U.S. Geological Survey Contract 14-08-0001-18257, $389 \mathrm{p}$.

Wilson, L.G., Herbert, R.A., and Ramsey, C.R., 1975, Transformations in quality of recharging effluent in the Santa Cruz River, in Hydrology and Water Resources in Arizona and the Southwest: American Water Resources Association, Arizona Section and Arizona Academy of Science, Hydrology Section Symposium Proceedings, Tempe, Arizona, v. 5, p. 169-176. 


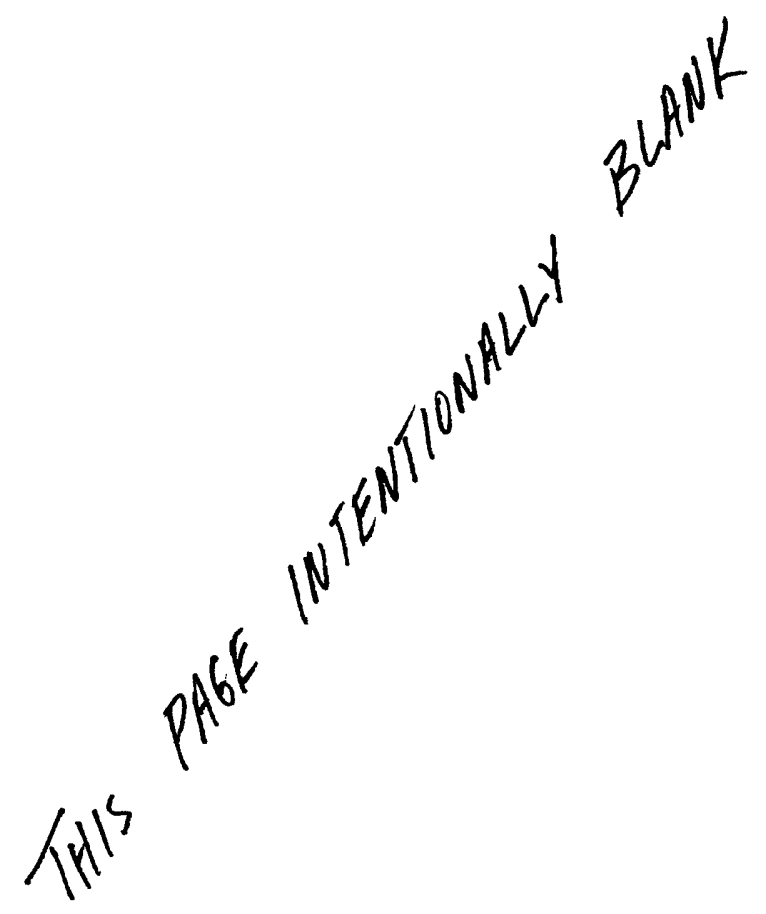

0.36 


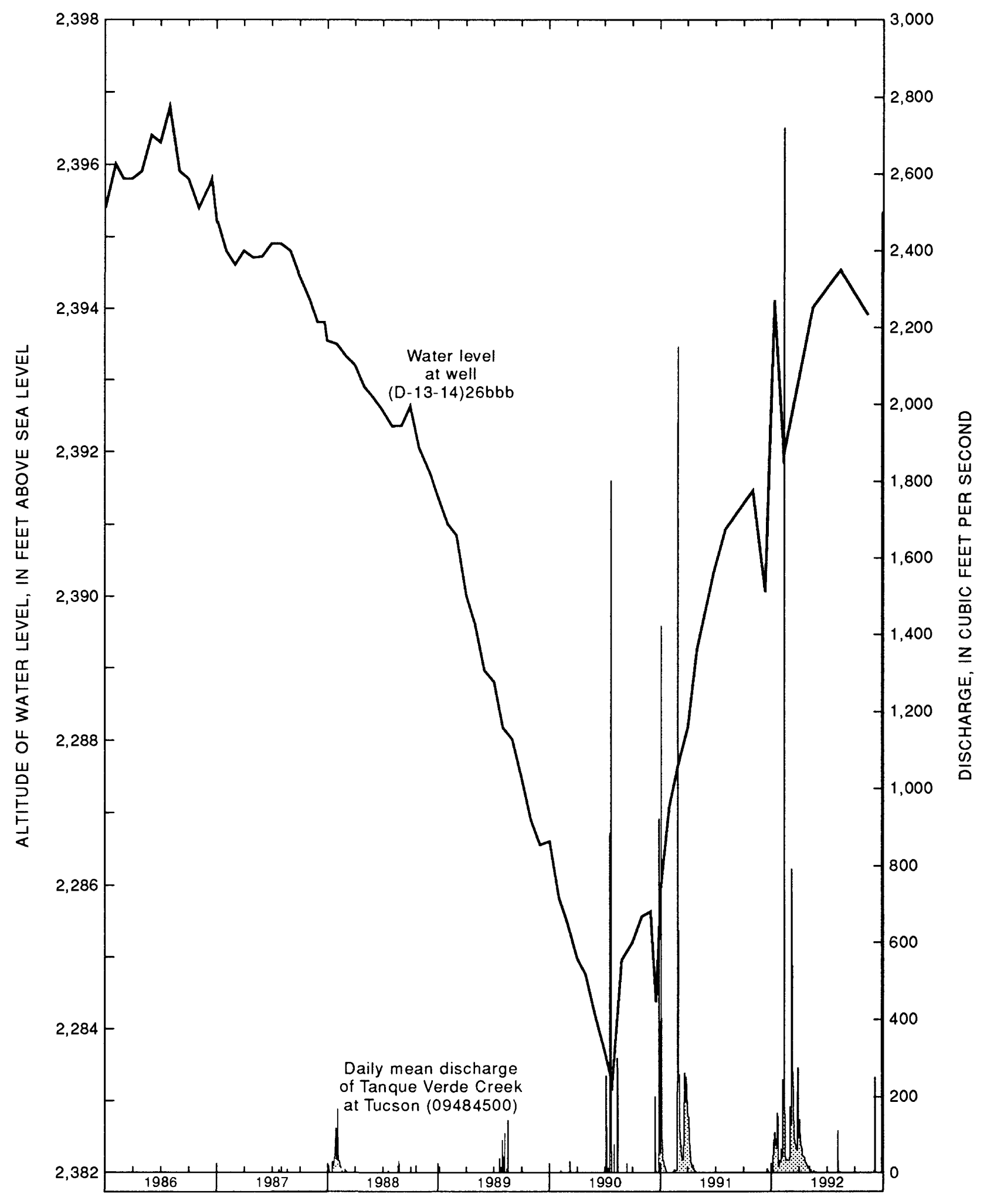

Figure 7. Flow in Tanque Verde Creek and water levels in observation well (D-13-14)26bbb, 1986-92. 


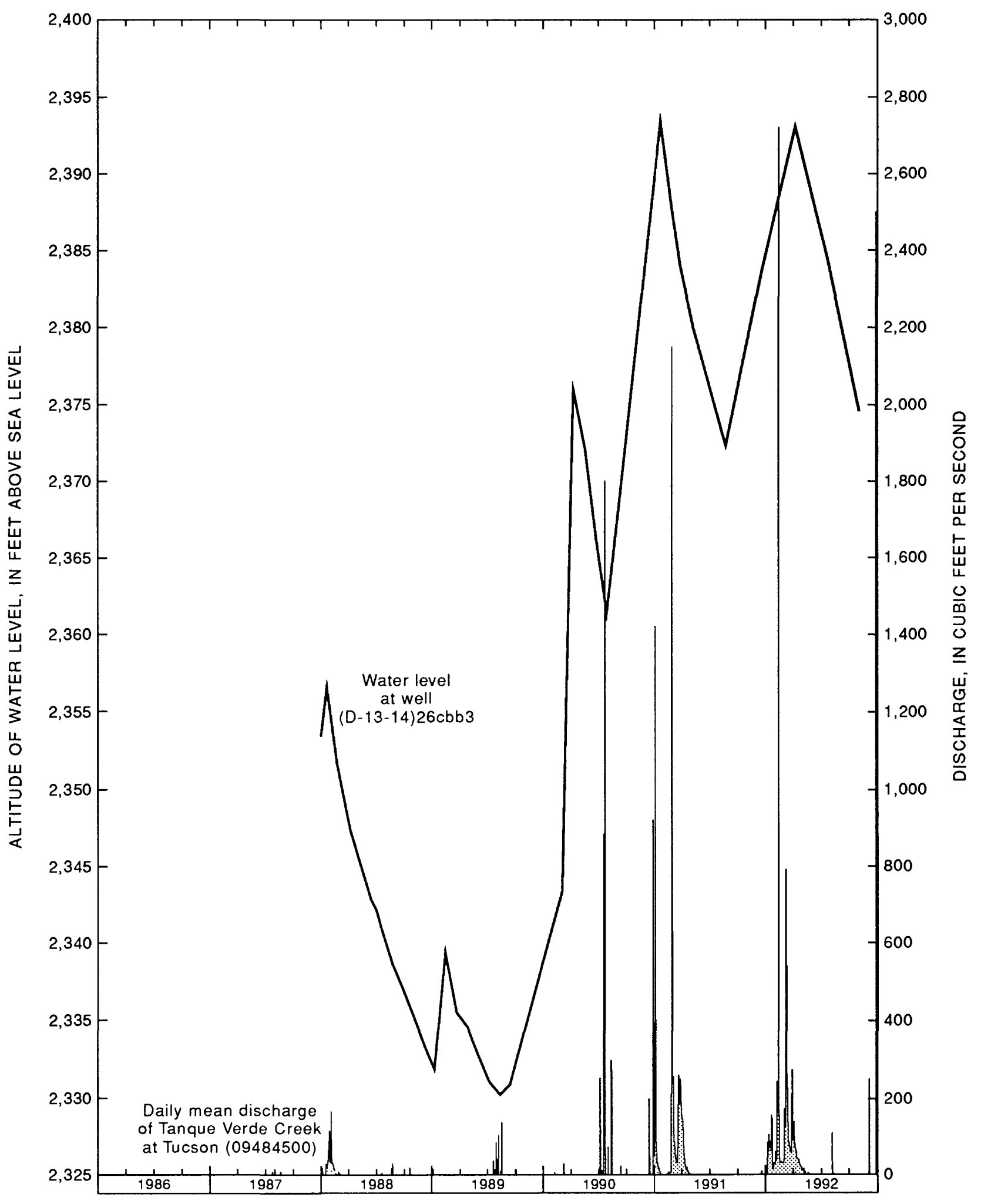

Figure 8. Flow in Tanque Verde Creek and water levels in observation well (D-13-14)26cbb3, 1986-92. 


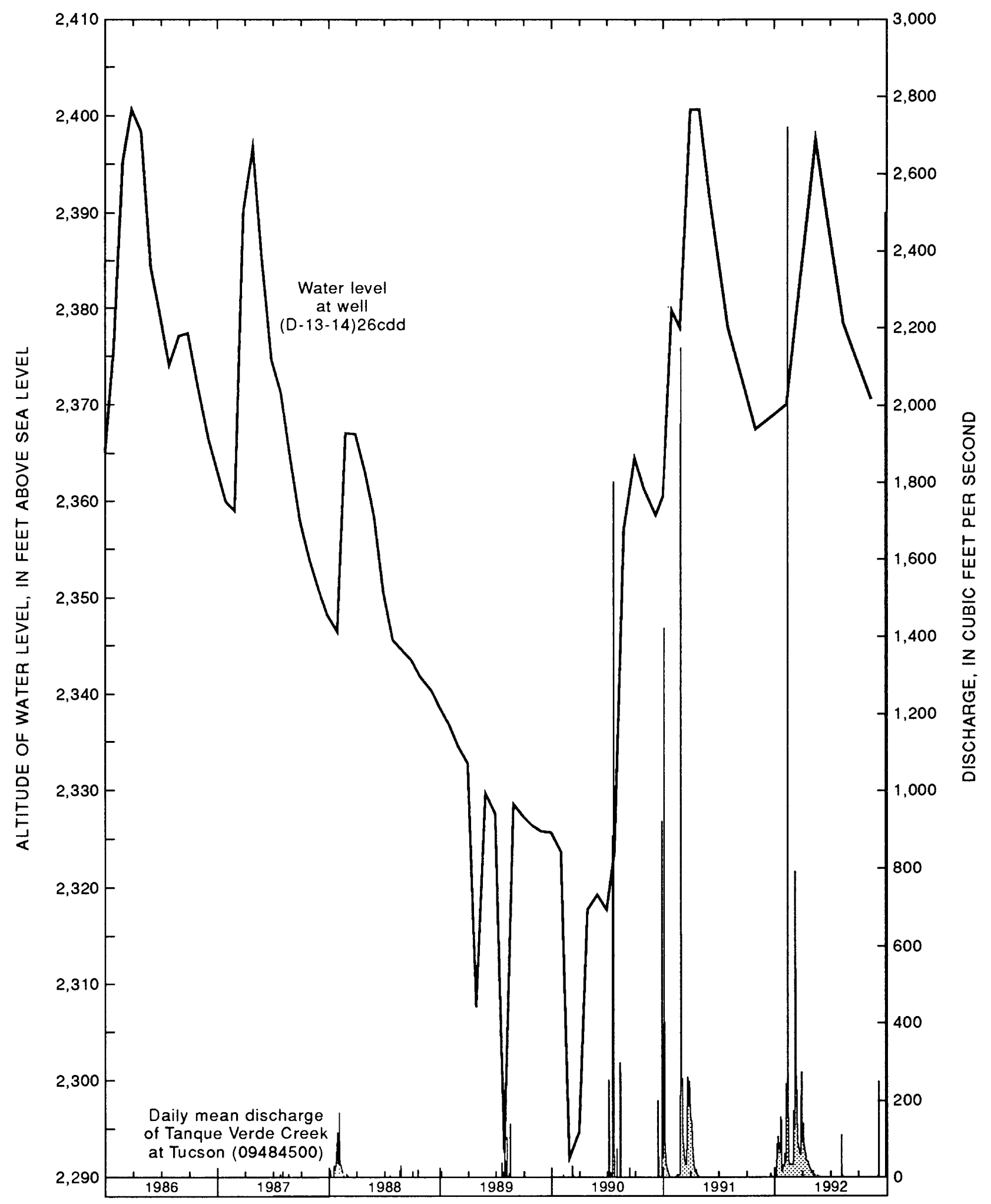

Figure 9. Flow in Tanque Verde Creek and water levels in observation well (D-13-14)26cdd, 1986-92. 


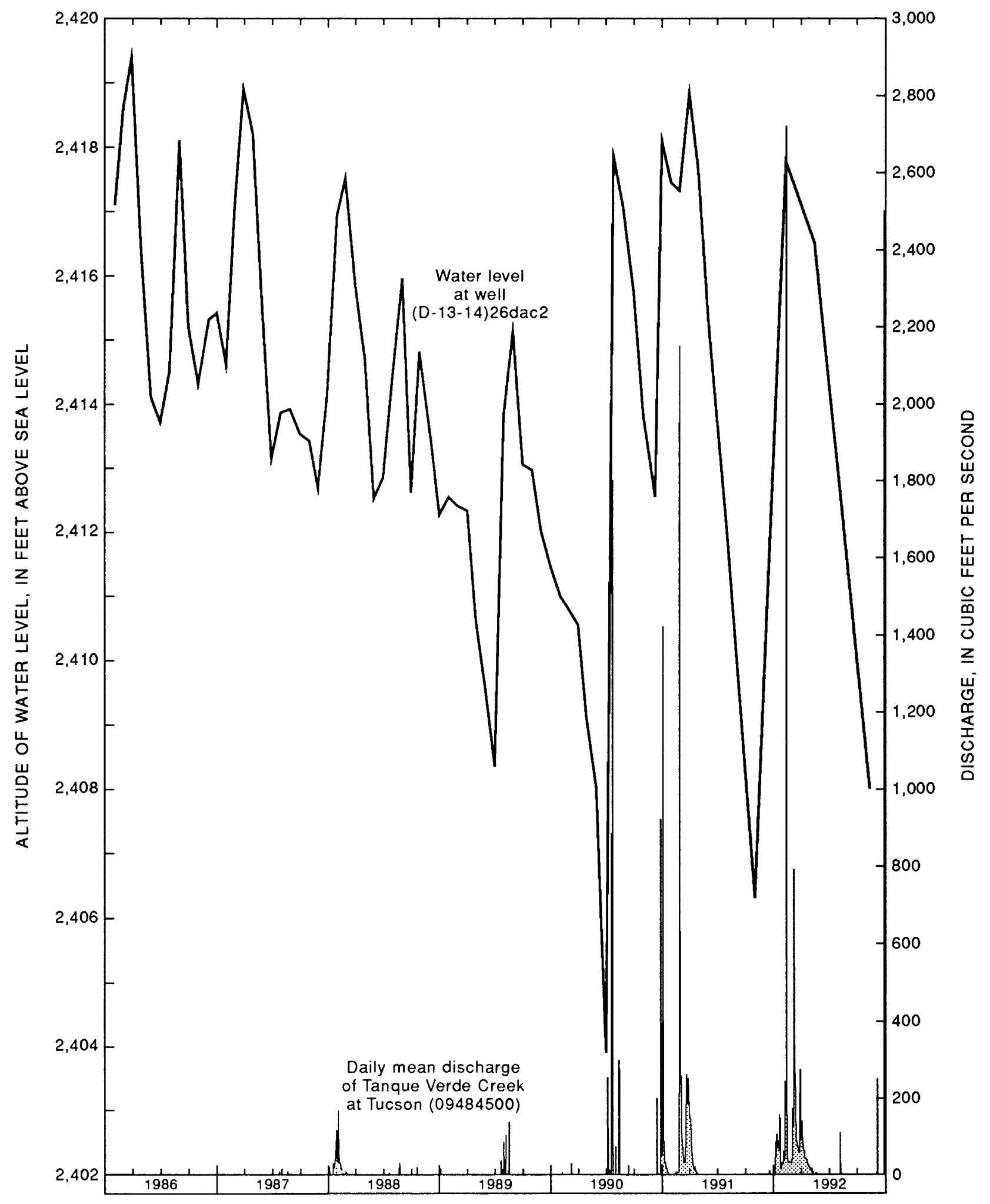

Figure 10. Flow in Tanque Verde Creek and water levels in observation well (D-13-14)26dac2, 1986-92. 


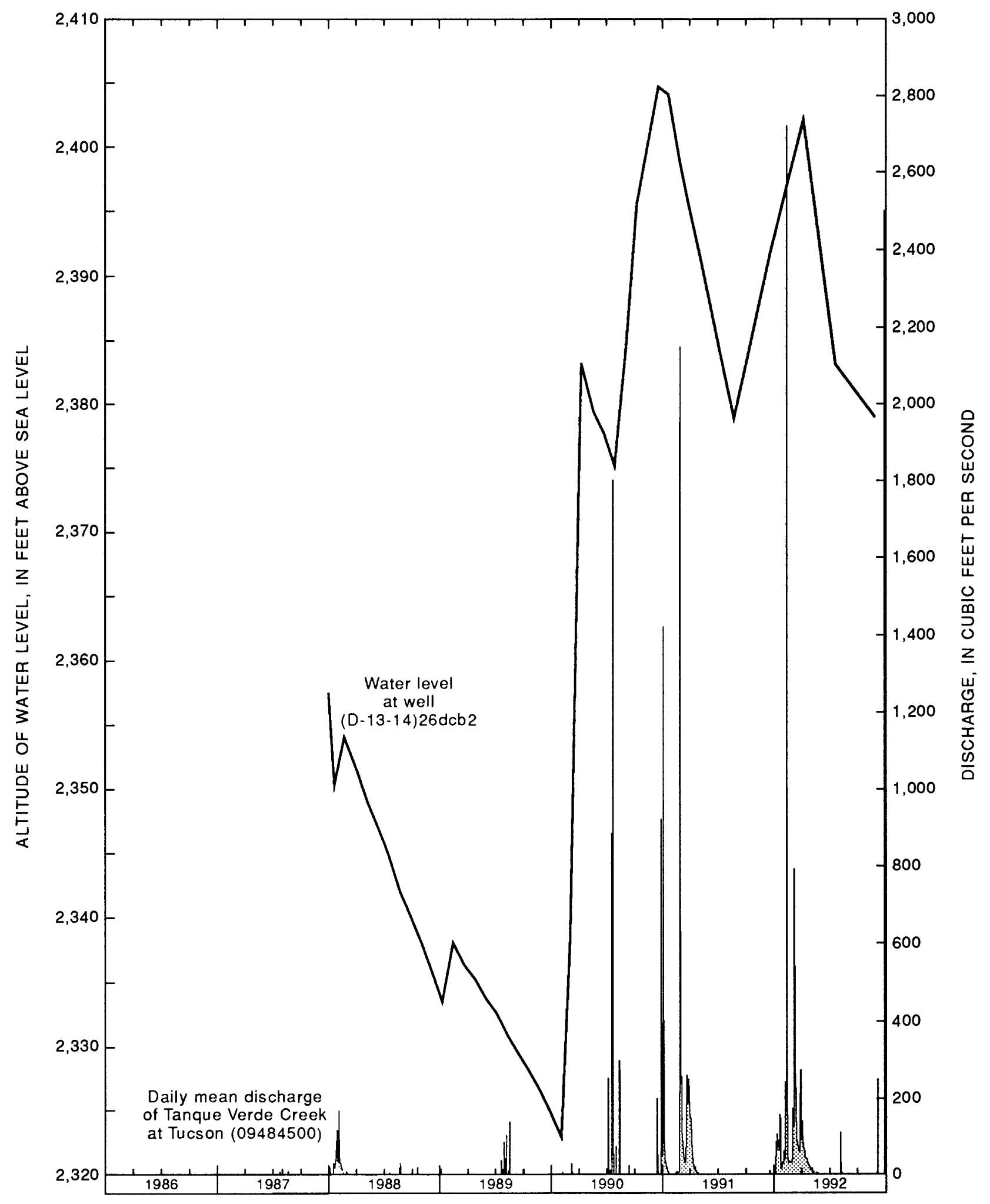

Figure 11. Flow in Tanque Verde Creek and water levels in observation well (D-13-14)26dcb2, 1986-92. 


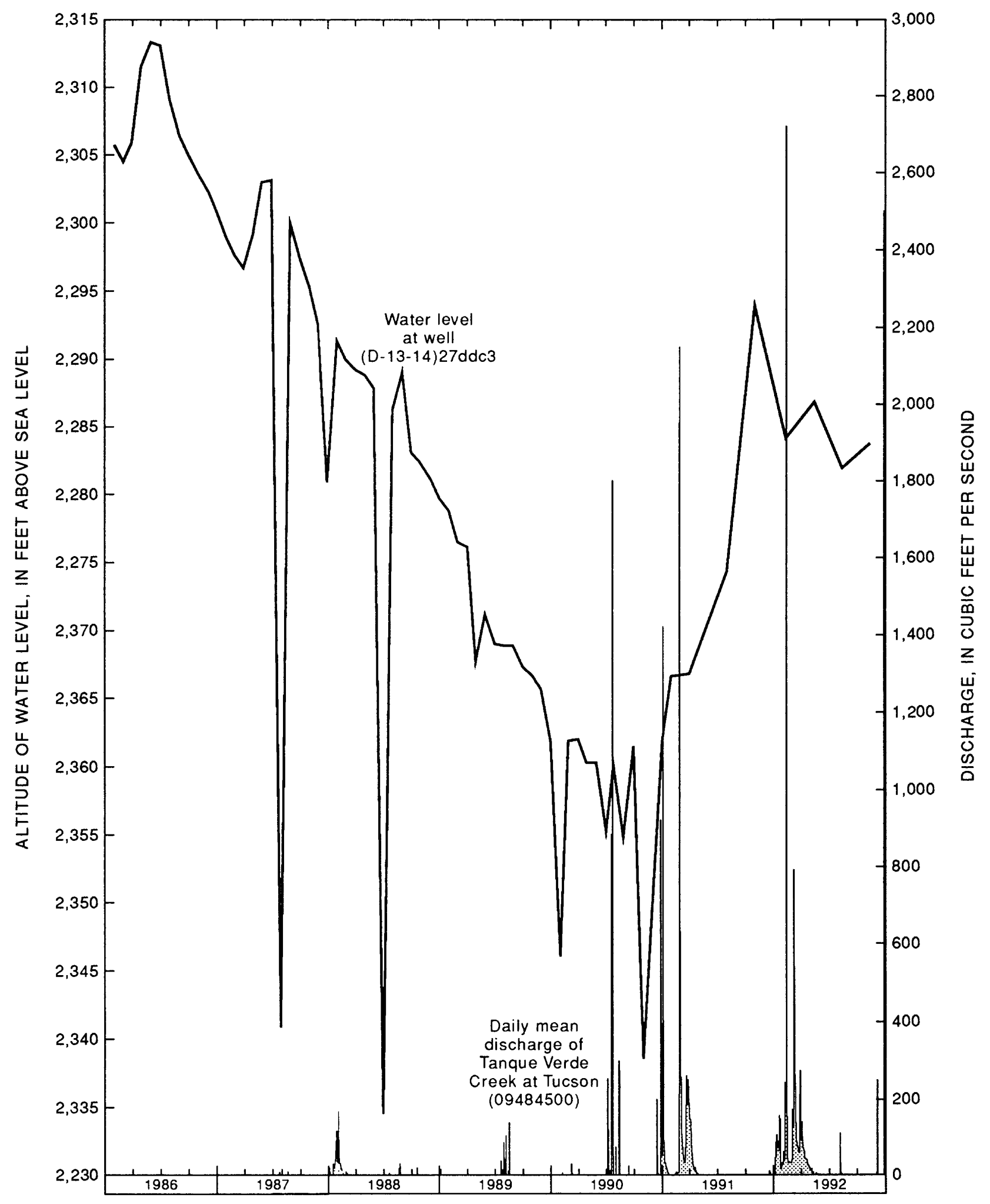

Figure 12. Flow in Tanque Verde Creek and water levels in observation well (D-13-14)27ddc3, 1986-92. 


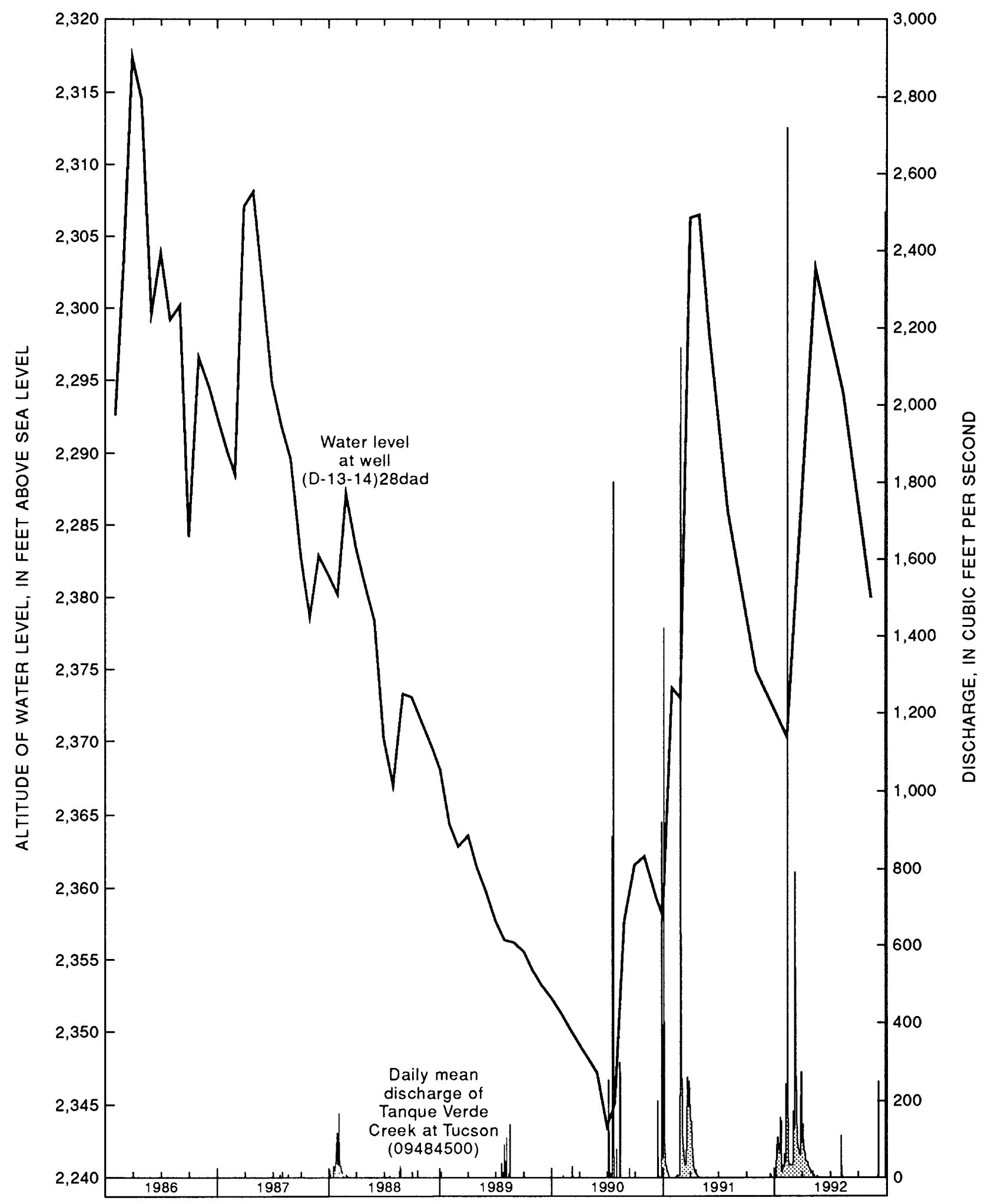

Figure 13. Flow in Tanque Verde Creek and water levels in observation well (D-13-14)28dad, 1986-92. 


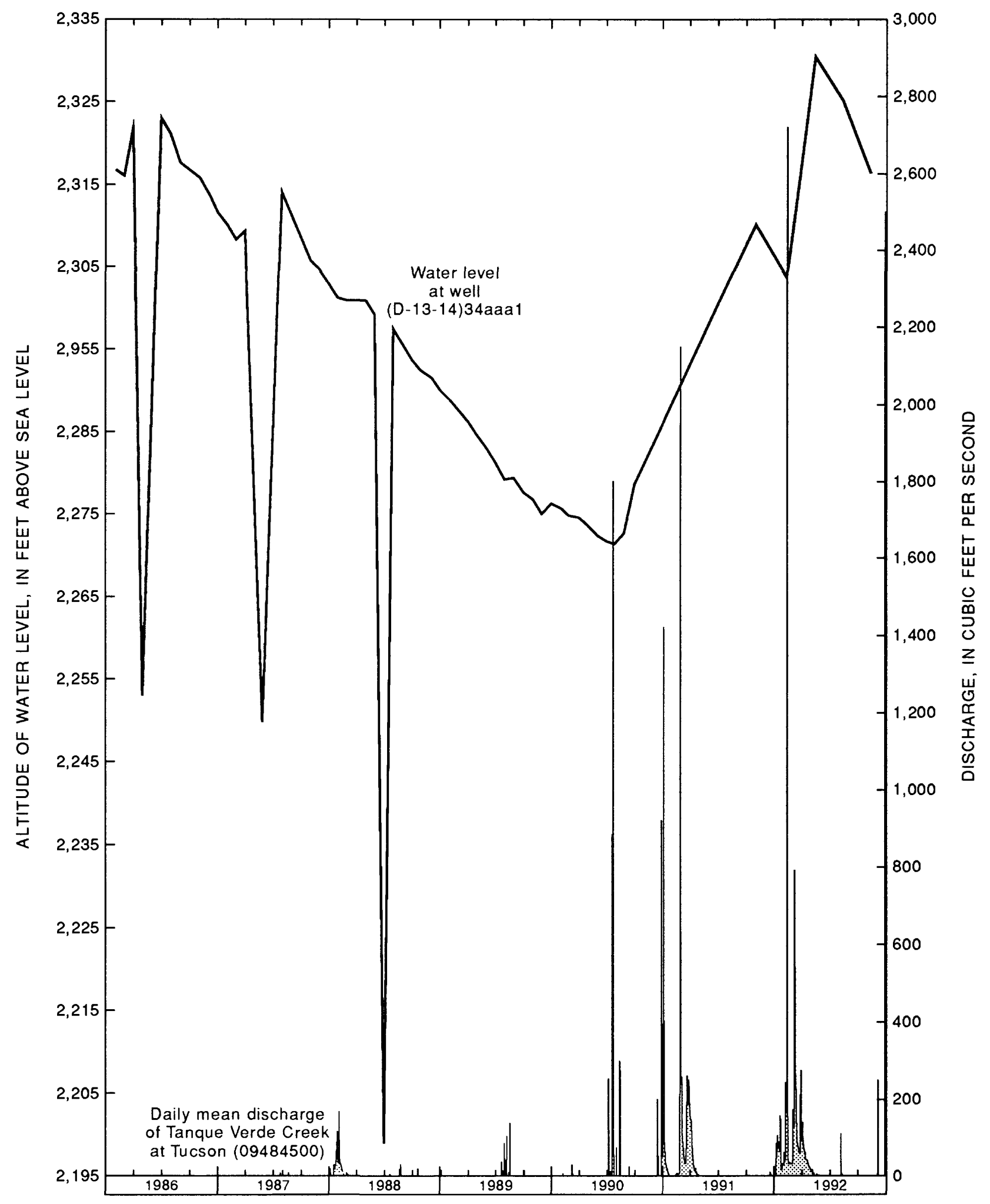

Figure 14. Flow in Tanque Verde Creek and water levels in observation well (D-13-14)34aaa1, 1986-92. 


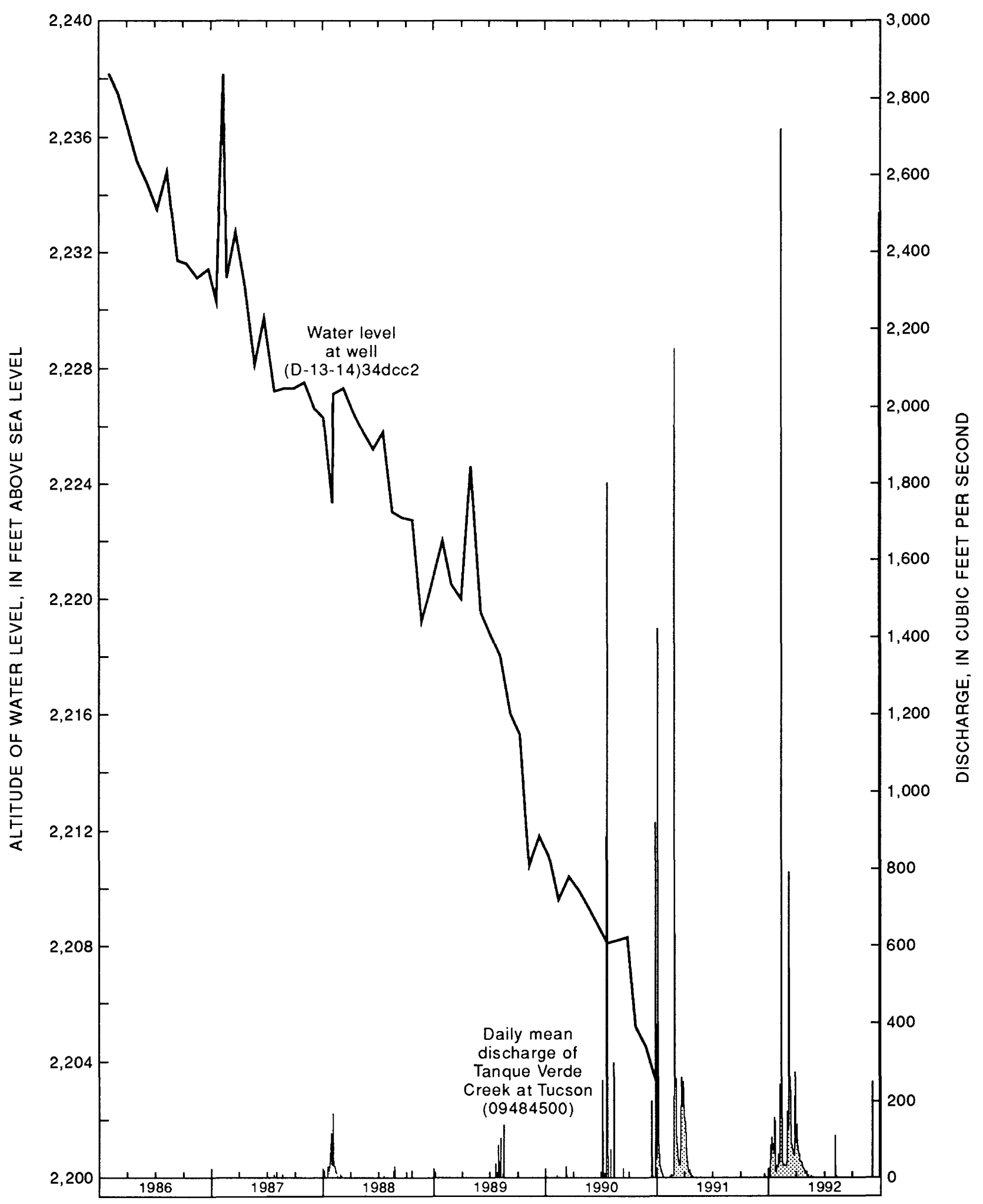

Figure 15. Flow in Tanque Verde Creek and water levels in observation well (D-13-14)34dcc2, 1986-92. 


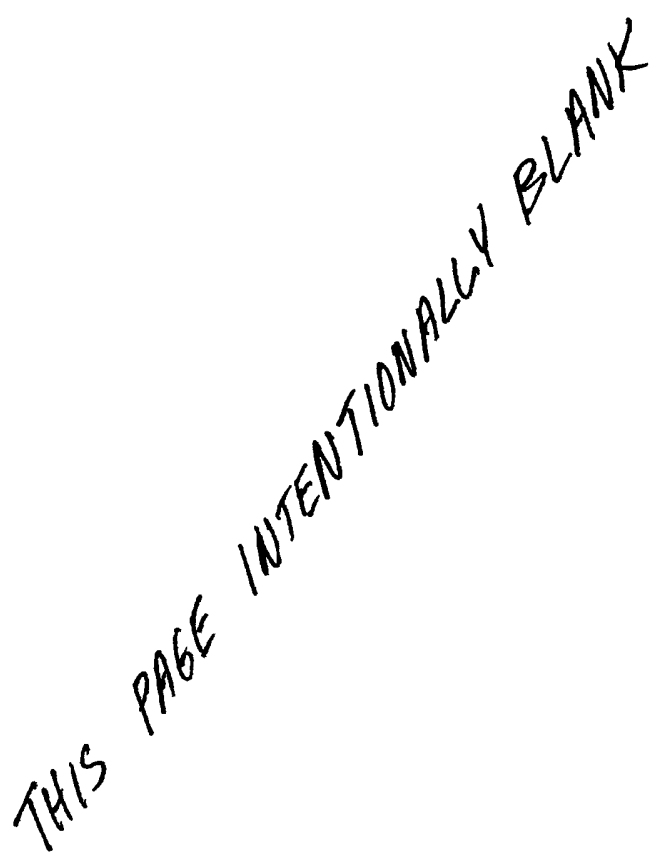

p. 46 
Table 7. Suspended-sediment concentration and particle-size distribution of surface-water samples, Rillito Creek basin

$\left[\mathrm{ft}^{3} / \mathrm{s}\right.$, cubic feet per second; $\mathrm{mg} / \mathrm{L}$, milligrams per liter, $\mathrm{mm}$, millimeter; $<$, less than; $\geq$, equal to or greater than; dashes indicate no data]

\begin{tabular}{|c|c|c|c|c|c|c|}
\hline \multirow[b]{2}{*}{ Date } & \multirow[b]{2}{*}{ Time } & \multirow[b]{2}{*}{$\begin{array}{l}\text { Sample-collection } \\
\text { method }\end{array}$} & \multirow[b]{2}{*}{$\begin{array}{c}\text { Discharge } \\
\left(\mathrm{ft}^{3} / \mathrm{s}\right)\end{array}$} & \multirow{2}{*}{$\begin{array}{l}\text { Suspended- } \\
\text { sediment } \\
\text { concentration } \\
\text { (mg/L) }\end{array}$} & \multicolumn{2}{|c|}{$\begin{array}{l}\text { Particle-size distribution, } \\
\text { in percent }\end{array}$} \\
\hline & & & & & $\begin{array}{l}\text { Silt and clay } \\
<0.062 \mathrm{~mm}\end{array}$ & $\begin{array}{l}\text { Sand and } \\
\text { coarser materiai } \\
\geq 0.062-2 \mathrm{~mm}\end{array}$ \\
\hline \multicolumn{7}{|c|}{ Tanque Verde Creek at Tucson } \\
\hline $08-25-87$ & 1535 & Automatic & 23 & 1,290 & 3 & 97 \\
\hline $01-28-88$ & 1600 & Manual & 71 & 47 & 71 & 29 \\
\hline $08-19-88$ & 1820 & Automatic & 1,690 & 10,300 & 92 & 8 \\
\hline $08-19-88$ & 1935 & Manual & 275 & 5,420 & 97 & 3 \\
\hline $08-03-89$ & 1735 & Manual & 69 & 3,740 & --- & --- \\
\hline $07-07-90$ & 1430 & Automatic & 2,400 & 9,020 & --- & --- \\
\hline $07-20-90$ & 0250 & Automatic & 1,600 & 5,520 & --- & --- \\
\hline $07-24-90$ & 0940 & Automatic & 4,400 & 8,380 & $\cdots$ & $\cdots$ \\
\hline $01-06-92$ & 1430 & Manual & 105 & 182 & 66 & 34 \\
\hline $01-13-92$ & 1230 & Manual & 62 & 22 & 78 & 22 \\
\hline $02-09-92$ & 1115 & Manual & 120 & 133 & 10 & 90 \\
\hline $02-21-92$ & 1100 & Manual & 35 & 39 & 24 & 76 \\
\hline $03-13-92$ & 1030 & Manual & 164 & 220 & 7 & 93 \\
\hline \multicolumn{7}{|c|}{ Pantano Wash at Broadway Boulevard } \\
\hline $07-20-88$ & 1520 & Manual & 20 & 4,860 & 100 & 0 \\
\hline $07-24-90$ & 1130 & Manual & 4,470 & 27,000 & --- & --- \\
\hline $02-13-92$ & 0930 & Manual & 97 & 3,340 & 8 & 92 \\
\hline $12-29-92$ & 1045 & Manual & 35 & 1,790 & 99 & 1 \\
\hline \multicolumn{7}{|c|}{ Alamo Wash at Glenn Street } \\
\hline $02-25-87$ & 1220 & Manual & 59 & 1,350 & 84 & 16 \\
\hline $07-26-87$ & 1515 & Manual & 168 & 2,930 & 83 & 17 \\
\hline $09-04-87$ & 1530 & Manual & 58 & 558 & 91 & 9 \\
\hline $11-01-87$ & 1000 & Automatic & 747 & 4,600 & 92 & 8 \\
\hline $08-20-88$ & 2200 & Automatic & 4,000 & 9,050 & 82 & 18 \\
\hline $07-20-90$ & 0330 & Automatic & 800 & 5,230 & -- & --- \\
\hline $07-24-90$ & 0700 & Automatic & 870 & 2,930 & --- & --- \\
\hline 09-14-90 & 1900 & Automatic & 955 & 6,260 & -- & --- \\
\hline $10-27-91$ & 1040 & Manual & 62 & 589 & 88 & 12 \\
\hline $11-15-91$ & 1320 & Manual & 33 & 147 & 90 & 10 \\
\hline $12-11-91$ & 1100 & Manual & 49 & 313 & 55 & 45 \\
\hline $01-06-92$ & 1310 & Manual & 325 & 789 & 64 & 36 \\
\hline \multicolumn{7}{|c|}{ Rillito Creek at Dodge Boulevard } \\
\hline $07-30-88$ & 0950 & Manual & 224 & 36,700 & 98 & 2 \\
\hline $08-20-88$ & 2340 & Automatic & 5,900 & 18,600 & 86 & 14 \\
\hline $10-20-88$ & 1000 & Manual & 265 & 5,410 & 84 & 16 \\
\hline 07-26-89 & 2030 & Manual & 3,940 & 26,300 & --- & --- \\
\hline $07-07-90$ & 1550 & Automatic & 2,300 & 25,200 & -- & --- \\
\hline $07-20-90$ & 0325 & Automatic & 4,800 & 15,400 & --- & --- \\
\hline $07-24-90$ & 1830 & Manual & 5,260 & 22,300 & --- & --- \\
\hline $02-09-92$ & 0950 & Manual & 95 & 251 & 47 & 53 \\
\hline $02-13-92$ & 1400 & Manual & 4,500 & 18,200 & 67 & 33 \\
\hline $02-13-92$ & 1433 & Automatic & 4,300 & 7,320 & 30 & 70 \\
\hline $03-13-92$ & 1430 & Manual & 149 & 67 & 35 & 65 \\
\hline
\end{tabular}


Tabie 8. Analytical results of surface-water samples, Rillito Creek basin Properties and major ions

$\left[\mathrm{ft}^{3} / \mathrm{s}\right.$, cubic feet per second; ${ }^{\circ} \mathrm{C}$, degrees Celsius; mm, millimeter, $\mu \mathrm{S} / \mathrm{cm}$, microsiemens per centimeter, $\mathrm{mg} / \mathrm{L}$, miligrams per liter, NTU nephelometric-turbidity units; $\mu \mathrm{g} / \mathrm{L}$, micrograms per liter, $\mathrm{pCi} / \mathrm{L}$, picocuries per liter, dashes indicate no data; <, less than]

\begin{tabular}{|c|c|c|c|c|c|c|c|c|c|}
\hline Date & $\begin{array}{c}\text { Discharge, } \\
\text { Instan- } \\
\text { taneous, } \\
\left(\mathrm{ft}^{3} / \mathrm{s}\right)\end{array}$ & $\begin{array}{l}\text { Temper- } \\
\text { ature, } \\
\text { water } \\
\left({ }^{\circ} \mathrm{C}\right)\end{array}$ & $\begin{array}{l}\text { Temper- } \\
\text { ature, } \\
\text { air } \\
\left({ }^{\circ} \mathrm{C}\right)\end{array}$ & $\begin{array}{c}\text { Baro- } \\
\text { metric } \\
\text { pressure } \\
(\mathrm{mm} \text { of } \\
\mathrm{Hg})\end{array}$ & $\begin{array}{c}\text { Specific } \\
\text { conduct- } \\
\text { ance } \\
(\mu \mathrm{S} / \mathrm{cm})\end{array}$ & $\begin{array}{c}\text { Specific } \\
\text { conduct- } \\
\text { ance } \\
\text { lab } \\
(\mu \mathrm{S} / \mathrm{cm})\end{array}$ & $\begin{array}{l}\text { Oxygen, } \\
\text { dis- } \\
\text { solved } \\
\text { (mg/L) }\end{array}$ & $\begin{array}{c}\text { pH } \\
\text { (standard } \\
\text { units) }\end{array}$ & $\begin{array}{c}\text { Turbidity } \\
\text { (NTU) }\end{array}$ \\
\hline \multicolumn{10}{|c|}{ Tanque Verde Creek at Tucson } \\
\hline $08-25-87$ & 23 & 28.0 & --- & 711 & 99 & 78 & 11.0 & 8.0 & 26 \\
\hline $01-28-88$ & 71 & 13.0 & 25.0 & $\cdots$ & 70 & 63 & --- & 8.0 & 6.5 \\
\hline $02-02-88$ & 44 & 9.0 & 12.0 & 711 & 59 & 62 & 10.2 & 9.2 & 4.4 \\
\hline $08-19-88$ & 1,800 & 26.0 & -- & --- & 135 & 108 & --- & 8.6 & 260 \\
\hline $08-19-88$ & 633 & 26.0 & --- & 692 & 109 & 107 & 6.5 & 8.7 & 380 \\
\hline 08-01-89 & 6 & 30.5 & 346.0 & 693 & 110 & 105 & 6.6 & 8.4 & 2.3 \\
\hline $07-07-90$ & 2,400 & --- & --- & -.. & --- & 257 & $-\cdots$ & --- & 330 \\
\hline $07-07-90$ & 450 & 23.5 & $\cdots$ & 681 & 290 & 192 & 6.4 & 8.2 & 1,200 \\
\hline $07-08-90$ & 1,600 & --- & --- & $\cdots$ & --- & 362 & --- & --- & 880 \\
\hline $01-05-91$ & 1,080 & --- & --- & -- & 98 & 215 & --- & 8.1 & 730 \\
\hline 01-06-92 & 105 & 11.0 & 10.0 & --- & 67 & 79 & -- & 7.0 & 46 \\
\hline $01-13-92$ & 62 & 9.0 & 9.5 & --- & 58 & 60 & --- & 6.9 & 5.2 \\
\hline 02-09-92 & 120 & 10.5 & 14.0 & --- & 47 & 51 & --- & 6.5 & 7.0 \\
\hline $02-21-92$ & 35 & --- & --- & $-\cdots$ & --- & 74 & --- & -- & 5.2 \\
\hline \multicolumn{10}{|c|}{ Pantano Wash at Broadway Boulevard } \\
\hline $11-01-87$ & 50 & 20.0 & 22.0 & 628 & 95 & 98 & 8.5 & 8.6 & 870 \\
\hline $07-24-90$ & 9,600 & --- & -- & --- & 118 & 106 & --- & 8.6 & 3,400 \\
\hline $07-24-90$ & 4,470 & 22.0 & 30.0 &.-- & 130 & 169 &.-- & 8.4 & 3,800 \\
\hline $02-13-92$ & 97 & -.- & --- & --- & 87 & --- & --- & --- & 850 \\
\hline $12-29-92$ & 35 & 13.0 & 17.0 & --- & 104 & 118 & -.- & 7.3 & 730 \\
\hline \multicolumn{10}{|c|}{ Alamo Wash at Glenn Street } \\
\hline $08-11-87$ & 230 & -- & 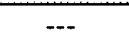 & $\cdots$ & 116 & 150 & --- & 7.9 & 87 \\
\hline $09-04-87$ & 58 & 27.0 & 28.0 & 712 & 95 & 172 & 6.2 & 8.5 & 140 \\
\hline $08-20-88$ & 4,000 & --- & --- & --- & 110 & 127 & --- & 8.6 & 160 \\
\hline $07-24-90$ & 870 & -- & --- & --- & --- & 188 & --- & $\ldots$ & 450 \\
\hline $10-27-91$ & 62 & 18.0 & 16.5 & -- & 97 & 108 & -- & 7.5 & 140 \\
\hline $11-15-91$ & 33 & 14.0 & 11.0 & --- & 79 & 112 & --- & 6.9 & 52 \\
\hline $12-11-91$ & 49 & 13.0 & 14.0 & --- & 65 & 93 & --- & 7.2 & 75 \\
\hline $01-06-92$ & 325 & 10.0 & 9.0 & --- & 56 & 87 & --- & 8.2 & 170 \\
\hline \multicolumn{10}{|c|}{ Rillito Creek at Dodge Boulevard } \\
\hline $07-30-88$ & 5,300 & $\cdots$ & $\cdots$ & --- & 380 & 461 & $\ldots$ & 8.0 & 29,000 \\
\hline $08-20-88$ & 5,900 & -.- & -.- & -- & 150 & 104 &.- & 8.7 & 500 \\
\hline $07-24-90$ & 1,360 & --- & --- & $\cdots$ & 475 & 293 & --- & --- & 1,300 \\
\hline $07-24-90$ & 5,260 & --- & 35.0 & --- & --- & 189 & --- & 8.5 & 3,200 \\
\hline $01-05-91$ & 1,050 & --- & --- & --- & --- & 103 & --- & 8.5 & 850 \\
\hline $02-09-92$ & 95 & 9.0 & 9.5 & --- & 64 & 75 & --- & 6.9 & 39 \\
\hline $02-13-92$ & 4,500 & -- & -.- & --- & --- & 104 & --- & -- & 510 \\
\hline $02-13-92$ & 4,300 & 17.0 & --- & --- & 66 & 87 & --- & 6.8 & 450 \\
\hline
\end{tabular}


Table 8. Analytical results of surface-water samples, Rillito Creek basin-Continued Properties and major ions-Continued

\begin{tabular}{|c|c|c|c|c|c|c|c|c|c|}
\hline Date & $\begin{array}{c}\text { Alka- } \\
\text { linity, } \\
\text { lab } \\
(\mathbf{m g} / \mathrm{L} \\
\text { as } \\
\left.\mathrm{CaCO}_{3}\right)\end{array}$ & $\begin{array}{l}\text { Alkalinity, } \\
\text { water } \\
\text { dissolved } \\
\text { in fleld } \\
(\mathrm{mg} / \mathrm{L} \text { as } \\
\left.\mathrm{CaCO}_{3}\right)\end{array}$ & $\begin{array}{c}\text { Carbonate, } \\
\text { water } \\
\text { dissolved } \\
\text { in fleld } \\
(\mathrm{mg} / \mathrm{L} \text { as } \\
\left.\mathrm{CO}_{3}\right)\end{array}$ & $\begin{array}{c}\text { Blcar- } \\
\text { bonate, } \\
\text { water } \\
\text { dissolved } \\
\text { In fleld } \\
\text { (mg/L as } \\
\left.\mathrm{HCO}_{3}\right)\end{array}$ & $\begin{array}{c}\text { Hardness, } \\
\text { total } \\
(\mathrm{mg} / \mathrm{L} \text { as } \\
\left.\mathrm{CaCO}_{3}\right)\end{array}$ & $\begin{array}{l}\text { Hardness, } \\
\text { noncar- } \\
\text { bonate } \\
\text { dlssolved } \\
\text { in fleld } \\
(\mathrm{mg} / \mathrm{L} \text { as } \\
\left.\mathrm{CaCO}_{3}\right)\end{array}$ & $\begin{array}{c}\text { Calclum, } \\
\text { dissolved } \\
\text { (mg/L as } \\
\text { Ca) }\end{array}$ & $\begin{array}{c}\text { Magne- } \\
\text { slum, } \\
\text { dissolved } \\
\text { (mg/L as } \\
\text { Mg) }\end{array}$ & $\begin{array}{l}\text { Potassium, } \\
\text { dissolved } \\
\text { (mg/L as K) }\end{array}$ \\
\hline \multicolumn{10}{|c|}{ Tanque Verde Creek at Tucson } \\
\hline $08-25-87$ & 26 & 30 & 0 & 36 & 25 & 0 & 7.7 & 1.4 & 1.5 \\
\hline $01-28-88$ & 14 & 15 & 0 & 19 & 18 & 2 & 5.4 & 1.1 & .8 \\
\hline $02-02-88$ & 14 & 34 & 11 & 20 & 21 & 0 & 6.1 & 1.3 & .9 \\
\hline $08-19-88$ & 32 & -.. & --- & --- & 35 & --- & 12 & 1.1 & 3.7 \\
\hline $08-19-88$ & 33 & 31 & 0 & 38 & 37 & 6 & 13 & 1.1 & 4.6 \\
\hline $08-02-89$ & 31 & 50 & 0 & 61 & 35 & 0 & 11 & 1.9 & 2.1 \\
\hline $07-07-90$ & 80 & --- & -- & --- & 81 & -- & 25 & 4.6 & 6.5 \\
\hline $07-07-90$ & 80 & 56 & 0 & 68 & 49 & 0 & 16 & 2.2 & 3.8 \\
\hline $07-08-90$ & 94 & --- & -- & --- & 51 & -.- & 17 & 2.1 & 4.0 \\
\hline $01-05-92$ & 90 & 40 & 0 & 49 & 39 & 0 & 13 & 1.7 & 1.9 \\
\hline $01-06-92$ & 27 & 21 & 0 & 26 & 25 & 3 & 8.2 & 1.0 & .9 \\
\hline $01-13-92$ & 15 & 12 & 0 & 15 & 16 & 3 & 4.7 & 1.0 & .7 \\
\hline $02-19-92$ & 15 & 12 & -- & 14 & 15 & 3 & 4.3 & 1.0 & .7 \\
\hline $02-21-92$ & 20 & --- & -- & --- & 21 & --- & 6.6 & 1.2 & .7 \\
\hline \multicolumn{10}{|c|}{ Pantano Wash at Broadway Boulevard } \\
\hline $11-01-87$ & 91 & 39 & 0 & 47 & 32 & 0 & 11 & 1.2 & 3.4 \\
\hline 07-24-88 & --- & 40 & -. & 49 & 36 & 0 & 12 & 1.5 & 2.9 \\
\hline $07-24-90$ & 134 & 50 & -- & 61 & 46 & $\mathbf{0}$ & 15 & 2.0 & 3.5 \\
\hline $02-13-92$ & 33 & -- & -- & --- & 28 & -- & 10 & .8 & 1.6 \\
\hline $12-29-92$ & 68 & 45 & 0 & 55 & 43 & 0 & 15 & 1.3 & 2.2 \\
\hline \multicolumn{10}{|c|}{ Alamo Wash at Glenn Street } \\
\hline $08-11-87$ & 39 & 48 & 0 & 56 & 43 & 0 & 15 & 1.3 & 2.8 \\
\hline $09-04-87$ & 73 & 39 & 0 & 47 & 150 & 110 & 53 & 5.1 & 2.1 \\
\hline $08-20-88$ & 28 & -- & -- & --- & 32 & -- & 11 & 1.0 & 3.0 \\
\hline $07-24-90$ & 87 & --- & -- & --- & 31 & -- & 11 & .9 & 1.6 \\
\hline $10-27-91$ & 71 & 27 & 0 & 33 & 37 & 10 & 13 & 1.0 & 2.9 \\
\hline $11-15-91$ & 43 & 25 & 0 & 31 & 31 & 5 & 11 & .8 & 2.0 \\
\hline $12-11-91$ & 52 & 28 & 0 & 34 & 26 & 0 & 9.4 & .7 & 1.4 \\
\hline $01-06-92$ & 43 & 25 & 0 & 31 & 24 & 0 & 8.5 & .6 & 1.3 \\
\hline \multicolumn{10}{|c|}{ Rillito Creek at Dodge Boulevard } \\
\hline $07-30-88$ & 109 & 97 & $\begin{array}{lll}0 & & \\
\end{array}$ & 121 & 140 & 40 & 46 & 5.9 & 5.1 \\
\hline $08-20-88$ & 39 & -- & --- & --- & 38 & -- & 13 & 1.4 & 4.2 \\
\hline $07-24-90$ & 35 & 30 & --- & 36 & 36 & 7 & 12 & 1.5 & 2.6 \\
\hline $07-24-90$ & 37 & 61 & -- & 74 & 52 & 0 & 16 & 2.9 & 4.1 \\
\hline $01-05-92$ & 46 & 47 & 0 & 57 & 42 & 0 & 14 & 1.6 & 1.9 \\
\hline $02-09-92$ & 29 & 23 & -- & 28 & 24 & 1 & 8.2 & .8 & .8 \\
\hline $02-13-92$ & 57 & --- & --- & --- & 30 & --- & 10 & 1.1 & 1.5 \\
\hline $02-13-92$ & 40 & 24 & 0 & 29 & 27 & ... & 9.1 & 1.1 & 1.3 \\
\hline
\end{tabular}


Table 8. Analytical results of surface-water samples, Rillito Creek basin-Continued Properties and major ions-Continued

\begin{tabular}{|c|c|c|c|c|c|c|c|c|}
\hline Date & $\begin{array}{l}\text { Sodium, } \\
\text { dissolved } \\
\text { (mg/L as } \mathrm{Na})\end{array}$ & $\begin{array}{l}\text { Sodium } \\
\text { adsorption } \\
\text { ratio }\end{array}$ & $\begin{array}{l}\text { Sodium, } \\
\text { percent }\end{array}$ & $\begin{array}{l}\text { Chloride, } \\
\text { dissolved } \\
\text { (mg/L as CL) }\end{array}$ & $\begin{array}{l}\text { Sulfate, } \\
\text { dissolved } \\
\text { (mg/L as } \\
\left.\mathrm{SO}_{4}\right)\end{array}$ & $\begin{array}{l}\text { Fluorlde, } \\
\text { dissolved } \\
\text { (mg/L as F) }\end{array}$ & $\begin{array}{c}\text { Silica, } \\
\text { dissolved } \\
\text { (mg/L as } \\
\left.\mathrm{SiO}_{2}\right)\end{array}$ & $\begin{array}{l}\text { Solids, } \\
\text { residue at } \\
180^{\circ} \mathrm{C} \\
\text { dissolved } \\
\text { (mg/L) }\end{array}$ \\
\hline \multicolumn{9}{|c|}{ Tanque Verde Creek at Tucson } \\
\hline $08-25-87$ & 5.1 & 0.4 & 29 & 2.2 & 13 & 0.20 & 12 & 44 \\
\hline $01-28-88$ & 5.0 & .5 & 36 & 2.9 & 15 & .20 & 13 & 62 \\
\hline $02-02-88$ & 4.9 & .5 & 33 & 3.0 & 16 & .20 & 12 & 61 \\
\hline $08-19-88$ & 8.8 & .7 & 33 & 5.2 & 12 & .20 & 5.4 & 108 \\
\hline $08-19-88$ & 4.3 & .3 & 18 & 3.4 & 13 & .20 & 6.5 & 107 \\
\hline 08-01-89 & 6.4 & .5 & 27 & 4.4 & 14 & .10 & 13 & 85 \\
\hline $07-07-90$ & 8.9 & .4 & 18 & 7.2 & 9.7 & $<.10$ & 8.5 & 205 \\
\hline $07-07-90$ & 4.1 & .3 & 14 & 3.6 & 6.4 & .10 & 6.8 & 137 \\
\hline $07-08-90$ & 4.4 & .3 & 15 & 5.7 & 4.5 & .20 & 6.3 & 155 \\
\hline $01-05-91$ & 5.3 & .4 & 22 & 6.6 & 5.9 & $<.10$ & 8.1 & 148 \\
\hline $01-06-92$ & 4.8 & .4 & 29 & 2.6 & 5.5 & $<.10$ & 9.4 & 62 \\
\hline $01-13-92$ & 5.5 & .6 & 42 & 3.4 & 9.5 & .20 & 15 & 59 \\
\hline $02-09-92$ & 4.2 & .5 & 37 & 2.4 & 6.3 & .20 & 11 & 41 \\
\hline $02-21-92$ & 5.7 & .5 & 36 & 4.0 & 8.9 & .10 & 14 & 60 \\
\hline \multicolumn{9}{|c|}{ Pantano Wash at Broadway Boulevard } \\
\hline $11-01-87$ & 2.9 & .2 & 15 & 2.6 & 8.5 & .10 & 5.1 & 54 \\
\hline $07-24-90$ & 6.1 & .4 & 25 & 1.3 & 1.2 & .20 & 6.6 & 80 \\
\hline $07-24-90$ & 7.1 & .5 & 24 & 3.5 & 7.3 & $<.10$ & 7.9 & 126 \\
\hline $02-13-92$ & 1.8 & .1 & 11 & 0.9 & 2.6 & $<.10$ & 2.8 & 39 \\
\hline $12-29-92$ & 4.0 & .3 & 16 & 2.0 & 5.4 & .10 & 8.8 & 95 \\
\hline \multicolumn{9}{|c|}{ Alamo Wash at Glenn Street } \\
\hline $08-11-87$ & 5.5 & .4 & 21 & 3.2 & 16 & .10 & 4.6 & 85 \\
\hline $09-04-87$ & 2.5 & .1 & 3 & 1.3 & 13 & .10 & 36 & 68 \\
\hline $08-20-88$ & 3.5 & .3 & 18 & 4.5 & 10 & .10 & 4.9 & 127 \\
\hline $07-24-90$ & 2.1 & .2 & 12 & 1.7 & 2.6 & .10 & 3.0 & 89 \\
\hline $10-27-91$ & 3.5 & .3 & 16 & 2.8 & 7.1 & .10 & 2.4 & 94 \\
\hline $11-15-91$ & 3.2 & .3 & 17 & 3.9 & 4.2 & .20 & 2.2 & 56 \\
\hline 12-11-91 & 1.6 & .1 & 11 & .8 & 2.0 & $<.10$ & 1.9 & 53 \\
\hline $01-06-92$ & 1.5 & .1 & 11 & 1.3 & 2.3 & .10 & 1.7 & 46 \\
\hline \multicolumn{9}{|c|}{ Rillito Creek at Dodge Boulevard } \\
\hline $07-30-88$ & 14 & .5 & 17 & 5.3 & 52 & .60 & 14 & 243 \\
\hline $08-20-88$ & 4.9 & .3 & 20 & 2.4 & 10 & .10 & 8.2 & 104 \\
\hline $07-24-90$ & 3.9 & .3 & 18 & 4.9 & 6.1 & $<.10$ & 4.7 & 84 \\
\hline $07-24-90$ & 7.5 & .5 & 22 & 4.6 & 17 & .20 & 18 & 163 \\
\hline $01-05-91$ & 6.2 & .4 & 23 & 3.8 & 7.2 & $<.10$ & 12 & 19 \\
\hline $02-09-92$ & 4.3 & .4 & 27 & 2.6 & 6.6 & .20 & 11 & 48 \\
\hline $02-13-92$ & 5.2 & .4 & 26 & 1.7 & 6.7 & .20 & 11 & 90 \\
\hline $02-13-92$ & 4.5 & .4 & 25 & 1.5 & 6.6 & .20 & 10 & 75 \\
\hline
\end{tabular}


Table 8. Analytical results of surface-water samples, Rillito Creek basin Nutrients

\begin{tabular}{|c|c|c|c|c|c|c|c|c|}
\hline Date & $\begin{array}{c}\text { Discharge, } \\
\text { instan- } \\
\text { taneous, } \\
\left(\mathrm{ft}^{3} / \mathrm{s}\right)\end{array}$ & $\begin{array}{c}\text { Phosphate, } \\
\text { total } \\
(\mathrm{mg} / \mathrm{L} \\
\left.\text { as } \mathrm{PO}_{4}\right)\end{array}$ & $\begin{array}{l}\text { Phosphate, } \\
\text { ortho, } \\
\text { dissolved } \\
\text { (mg/L } \\
\left.\text { as } \mathrm{PO}_{4}\right)\end{array}$ & $\begin{array}{c}\text { Phos- } \\
\text { phorus, } \\
\text { total } \\
\text { (mg/L } \\
\text { as P) }\end{array}$ & $\begin{array}{l}\text { Phos- } \\
\text { phorus, } \\
\text { dissolved } \\
\text { (mg/L } \\
\text { as P) }\end{array}$ & $\begin{array}{l}\text { Phosphate, } \\
\text { ortho, } \\
\text { dissolved } \\
\text { (mg/L as P) }\end{array}$ & $\begin{array}{c}\text { Nitrogen, } \\
\text { total } \\
\text { (mg/L } \\
\text { as N) }\end{array}$ & $\begin{array}{c}\text { Nitrogen, } \\
\text { organic } \\
\text { total } \\
\text { (mg/Las } N)\end{array}$ \\
\hline \multicolumn{9}{|c|}{ Tanque Verde Creek at Tucson } \\
\hline $08-25-87$ & 23 & 0.06 & 0.06 & 0.10 & 0.03 & 0.02 & --- & 0.67 \\
\hline $02-02-88$ & 44 & .03 & --- & .02 & .01 & $<.01$ & -- & .57 \\
\hline 08-01-89 & 6 & .15 & .15 & .09 & .07 & .05 & -- & .58 \\
\hline 08-03-89 & 69 & 2.36 & 1.2 & 2.40 & .46 & .38 & 11 & 9.7 \\
\hline $01-05-91$ & 1,080 & .34 & .28 & .59 & .29 & .09 & 1.3 & 1.1 \\
\hline $01-06-92$ & 105 & .18 & .06 & .18 & .03 & .02 & .71 & .54 \\
\hline $01-13-92$ & 62 & .03 & .03 & .02 & .02 & .01 & .46 & .37 \\
\hline $02-21-92$ & 35 & .06 & .06 & .02 & .02 & .02 & .31 & .17 \\
\hline \multicolumn{9}{|c|}{ Pantano Wash at Broadray Boulevard } \\
\hline $11-01-87$ & 50 & .67 & .43 & 1.20 & .18 & .14 & 1.1 & .63 \\
\hline $07-24-90$ & 4,470 & .43 & .43 & 4.90 & .26 & .14 & 3.3 & 2.6 \\
\hline $02-13-92$ & 97 & .34 & .28 & .49 & .09 & .09 & 1.2 & .89 \\
\hline $12-29-92$ & 35 & --- & .28 & --- & -- & .09 & --- & --- \\
\hline \multicolumn{9}{|c|}{ Alamo Wash at Glenn Street } \\
\hline $09-04-87$ & 58 & .40 & .25 & .16 & .12 & .08 & 2.2 & 1.5 \\
\hline $10-27-91$ & 62 & .80 & .71 & .65 & .33 & .23 & 3.2 & 1.9 \\
\hline $11-15-91$ & 33 & .46 & .34 & .29 & .14 & .11 & 1.5 & .75 \\
\hline $12-11-91$ & 49 & .37 & .28 & .20 & .09 & .09 & .95 & .51 \\
\hline $01-06-92$ & 325 & .71 & .25 & .59 & .09 & .08 & .73 & .26 \\
\hline \multicolumn{9}{|c|}{ Rilito Creek at Dodge Boulevard } \\
\hline $07-30-88$ & 5,300 & .40 & .15 & 1.20 & .07 & .05 & 4.0 & 1.6 \\
\hline $07-26-89$ & 3,940 & 1.38 & .98 & .52 & .36 & .32 & 4.4 & 3.0 \\
\hline $01-05-91$ & 1,050 & .34 & .31 & .42 & .33 & .10 & 1.3 & .95 \\
\hline $02-09-92$ & 95 & .18 & .06 & .14 & .01 & .02 & --- & .52 \\
\hline $02-13-92$ & 4,500 & .61 & .25 & 1.00 & .09 & .08 & 2.3 & 1.9 \\
\hline $02-13-92$ & 4,300 & .21 & .15 & .42 & .07 & .05 & 1.7 & 1.4 \\
\hline
\end{tabular}


Table 8. Analytical results of surface-water samples, Rillito Creek basin Nutrients-Continued

\begin{tabular}{|c|c|c|c|c|c|c|c|c|c|}
\hline Date & $\begin{array}{c}\text { Nitrogen, } \\
\text { ammonia } \\
\text { dis- } \\
\text { solved } \\
\text { (mg/L. } \\
\text { as N) }\end{array}$ & $\begin{array}{c}\text { Nitrogen, } \\
\text { ammonia } \\
\text { total } \\
\text { (mg/L. } \\
\text { as N) }\end{array}$ & $\begin{array}{l}\text { Nitro- } \\
\text { gen, } \\
\text { nitrite, } \\
\text { dis- } \\
\text { solved } \\
\text { (mg/L } \\
\text { as N) }\end{array}$ & $\begin{array}{l}\text { Nitro- } \\
\text { gen, } \\
\text { nitrite, } \\
\text { total } \\
\text { (mg/L } \\
\text { as N) }\end{array}$ & $\begin{array}{l}\text { Nitro- } \\
\text { gen, } \\
\text { nitrate, } \\
\text { dis- } \\
\text { soived } \\
\text { (mg/L } \\
\text { as N) }\end{array}$ & $\begin{array}{c}\text { Nitro- } \\
\text { gen, } \\
\text { nitrate, } \\
\text { total } \\
\text { (mg/L } \\
\text { as N) }\end{array}$ & $\begin{array}{l}\text { Nitrogen, } \\
\text { ammonia } \\
\text { plus } \\
\text { organic } \\
\text { total } \\
\text { (mg/L } \\
\text { as N) }\end{array}$ & $\begin{array}{c}\text { Nitrogen, } \\
\mathrm{NO}_{2}+\mathrm{NO}_{3} \\
\text { total } \\
\text { (mg/L as } \mathrm{N} \text { ) }\end{array}$ & $\begin{array}{l}\text { Nitrogen, } \\
\mathrm{NO}_{2}+\mathrm{NO}_{3} \\
\text { dissolved } \\
\text { (mg/L as } \mathrm{N} \text { ) }\end{array}$ \\
\hline \multicolumn{10}{|c|}{ Tanque Verde Creek at Tucson } \\
\hline $08-25-87$ & $<0.01$ & 0.03 & -- & $<0.01$ & --- & -- & 0.70 & $<0.10$ & $<0.10$ \\
\hline $02-02-88$ & .02 & .03 & --- & $<.01$ & --- & --- & .60 & $<.10$ & $<.10$ \\
\hline $08-01-89$ & .03 & .02 & --- & $<.01$ & --- & --- & .60 & $<.10$ & $<.10$ \\
\hline 08-03-89 & .32 & .25 & --- & .09 & $-\cdots$ & 0.71 & 10 & .80 & .91 \\
\hline $01-05-91$ & .50 & .04 & .02 & .02 & 0.18 & .18 & 1.1 & .20 & .20 \\
\hline $01-06-92$ & .04 & .06 & .01 & .03 & .08 & .0 & .60 & .11 & .09 \\
\hline $01-13-92$ & .05 & .03 & .02 & $<.01$ & .07 & -- & .40 & .06 & .08 \\
\hline $02-21-92$ & .05 & .03 & $<.01$ & .01 & --- & 10 & .20 & .11 & .11 \\
\hline \multicolumn{10}{|c|}{ Pantano Wash at Broadway Boulevard } \\
\hline $11-01-87$ & .09 & .07 & --- & .02 & --- & .38 & .70 & .40 & .34 \\
\hline $07-24-90$ & .15 & .08 & --- & .05 & --- & .55 & 2.70 & .60 & .50 \\
\hline $02-13-92$ & .04 & .01 & .02 & .02 & .26 & .26 & .90 & .28 & .28 \\
\hline $12-29-92$ & .05 & --- & .02 & --- & .12 & --- & -- & --- & .14 \\
\hline \multicolumn{10}{|c|}{ Alamo Wash at Glenn Street } \\
\hline $09-04-87$ & .14 & .33 & --- & .03 & $\cdots$ & .37 & 1.80 & .40 & .36 \\
\hline $10-27-91$ & .56 & .59 & .03 & .04 & .71 & .67 & 2.50 & .71 & .74 \\
\hline $11-15-91$ & .25 & .25 & .03 & .05 & .45 & .43 & 1.00 & .48 & .48 \\
\hline $12-11-91$ & $<.16$ & .09 & .02 & .04 & .36 & .31 & .60 & .35 & .38 \\
\hline $01-06-92$ & .09 & .14 & .02 & .12 & .31 & .21 & .40 & .33 & .33 \\
\hline \multicolumn{10}{|c|}{ Rillito Creek at Dodge Boulevard } \\
\hline $07-30-88$ & .05 & .97 & --- & .27 & --- & 1.13 & 2.60 & 1.40 & $<.10$ \\
\hline $07-26-89$ & .56 & .48 & --- & .12 & --- & .78 & 3.50 & .90 & .98 \\
\hline $01-05-91$ & .37 & .05 & .02 & .02 & .18 & .28 & 1.00 & .30 & .20 \\
\hline $02-09-92$ & .04 & .08 & $<.01$ & .02 & --- & --- & .60 & $<.05$ & $<.05$ \\
\hline $02-13-92$ & .06 & .06 & $<.01$ & $<.01$ & --- & --- & 2.00 & .26 & .28 \\
\hline $02-13-92$ & .04 & .07 & $<.01$ & $<.01$ & --- & --- & 1.50 & .20 & .19 \\
\hline
\end{tabular}


Table 8. Analytical results of surface-water samples, Rillito Creek basin Trace elements

\begin{tabular}{|c|c|c|c|c|c|c|c|c|c|}
\hline Date & $\begin{array}{c}\text { Discharge, } \\
\text { instan- } \\
\text { taneous, } \\
\left(\mathrm{ft}^{3} / \mathrm{s}\right)\end{array}$ & $\begin{array}{c}\text { Barium, } \\
\text { dissolved } \\
(\mu \mathrm{g} / \mathrm{L} \text { as } \\
\mathrm{Ba})\end{array}$ & $\begin{array}{c}\text { Barium, } \\
\text { total } \\
\text { recov- } \\
\text { erable } \\
(\mu \mathrm{g} / \mathrm{L} \text { as } \\
\mathrm{Ba})\end{array}$ & $\begin{array}{l}\text { Zinc, } \\
\text { dis- } \\
\text { solved } \\
(\mu \mathrm{g} / \mathrm{L} \\
\text { as Zn) }\end{array}$ & $\begin{array}{c}\text { Zinc, } \\
\text { total } \\
\text { recov- } \\
\text { erable } \\
(\mu g / L \\
\text { as Zn) }\end{array}$ & $\begin{array}{l}\text { Sele- } \\
\text { nium, } \\
\text { dis- } \\
\text { solved } \\
(\mu \mathrm{g} / \mathrm{L} \\
\text { as Se) }\end{array}$ & $\begin{array}{c}\text { Seie- } \\
\text { nium, } \\
\text { total } \\
\text { ( } \mu \mathrm{g} / \mathrm{L} \\
\text { as Se) }\end{array}$ & $\begin{array}{l}\text { Molyb- } \\
\text { denum, } \\
\text { dis- } \\
\text { solved } \\
\text { ( } \mu \mathrm{g} / \mathrm{L} \\
\text { as Mo) }\end{array}$ & $\begin{array}{c}\text { Molyb- } \\
\text { denum } \\
\text { total } \\
\text { ( } \mu \mathrm{g} / \mathrm{L} \\
\text { as Mo) }\end{array}$ \\
\hline \multicolumn{10}{|c|}{ Tanque Verde Creek at Tucson } \\
\hline $08-25-87$ & 23 & - & $<100$ & $\cdots$ & $<10$ & - & $<1$ & - & $<1$ \\
\hline $02-02-88$ & 44 & 100 & 100 & 20 & 20 & $<1$ & $<1$ & 2 & 2 \\
\hline $08-20-88$ & 3,800 & - & 1,800 & --- & 1,000 & --- & $<1$ & - & $<1$ \\
\hline 08-03-89 & 69 & - & 1,200 & - & 760 & $\ldots$ & $<1$ & - & 2 \\
\hline $08-18-89$ & 2,100 & - & 4,000 & -- & 3,300 & --- & $<4$ & - & $<4$ \\
\hline $07-07-90$ & 450 & -- & 2,000 & -- & 920 & -- & $<3$ & - & $<1$ \\
\hline 07-08-90 & 1,600 & $\ldots$ & 2,000 & -- & 1,300 & -- & $<3$ & - & 3 \\
\hline $07-20-90$ & 2,200 & 11 & 1,200 & 12 & 790 & $<1$ & $<2$ & 1 & 2 \\
\hline $07-24-90$ & 4,400 & - & 1,500 & -- & 800 & -.. & $<1$ & - & $<1$ \\
\hline $08-03-90$ & 1,400 & - & 1,700 & -- & 1,000 & -- & $<3$ & - & 1 \\
\hline $08-1490$ & 980 & $\ldots$ & 2,200 &.- & 1,500 & -- & $<2$ & -- & $<1$ \\
\hline $12-28-90$ & 1,000 & $\ldots$ & 1,200 & -- & 920 & --- & $<1$ & -- & $<1$ \\
\hline $01-05-91$ & 1,080 & - & 500 & -- & 450 & $\ldots$ & $<1$ & - & 3 \\
\hline $01-06-92$ & 105 & 29 & 100 & 11 & 60 & $<1$ & $<1$ & $<1$ & $<1$ \\
\hline $02-09-92$ & 120 & 6 & $<100$ & 7 & 20 & $<1$ & $<1$ & $<1$ & $<1$ \\
\hline $02-21-92$ & 35 & $<100$ & $<100$ & $<10$ & 20 & $<1$ & $<1$ & $<1$ & $<1$ \\
\hline \multicolumn{10}{|c|}{ Pantano Wash at Broadway Boulevard } \\
\hline $11-01-87$ & 50 & 14 & 1,000 & 12 & 380 & $<1$ & $<1$ & $<1$ & 1 \\
\hline $07-30-88$ & 26 & - & 3,000 & --- & 1,200 & -.. & $<1$ & - & 2 \\
\hline $07-24-90$ & 3,710 & 33 & 3,600 & 6 & 1,300 & $<1$ & $<2$ & 2 & 2 \\
\hline $07-24-90$ & 4,470 & 29 & 4,500 & 26 & 1,500 & $<1$ & $<3$ & 1 & 1 \\
\hline 08-03-90 & 5,300 & -- & 4,900 & -- & 1,800 & --- & $<3$ & -- & 2 \\
\hline $02-13-92$ & 97 & 16 & 800 & 5 & 360 & $<1$ & $<1$ & 1 & 1 \\
\hline $12-29-92$ & 35 & 30 & $\cdots$ & 5 & - & $<1$ & - & $<1$ & $\cdots$ \\
\hline \multicolumn{10}{|c|}{ Alamo Wash at Glenn Street } \\
\hline $02-25-87$ & 55 & $\cdots$ & 100 & -- & 80 & $\cdots$ & $<1$ & - & 3 \\
\hline $08-11-87$ & 230 & 29 & $\cdots$ & 11 & - & $<1$ & - & $<1$ & -- \\
\hline $09-0487$ & 58 & 190 & 200 & 170 & 190 & 5 & 5 & 1 & 1 \\
\hline $08-20-88$ & 4,000 & $<100$ & $<100$ & 110 & 1,300 & $<1$ & $<1$ & 1 & 2 \\
\hline $07-20-90$ & 629 & 24 & 1,000 & 5 & 530 & $<1$ & $<2$ & $<1$ & 1 \\
\hline $07-24-90$ & 870 & --- & 500 & --- & 270 & --- & $<1$ & - & 1 \\
\hline $09-14-90$ & 695 & -- & 600 & -- & 380 & --- & $<1$ & -- & 1 \\
\hline $10-27-91$ & 62 & $<100$ & 200 & 38 & 190 & $<1$ & $<1$ & 3 & 3 \\
\hline $11-15-91$ & 33 & 17 & $<100$ & 13 & 110 & $<1$ & $<1$ & 2 & 1 \\
\hline $12-11-91$ & 49 & 14 & $<100$ & 8 & 120 & $<1$ & $<1$ & 1 & $<1$ \\
\hline $01-06-92$ & 325 & 27 & 200 & 7 & 220 & $<1$ & $<1$ & 1 & 2 \\
\hline \multicolumn{10}{|c|}{ Rfilito Creek at Dodge Boulevard } \\
\hline $07-30-88$ & 5,300 & -- & 10,000 & $\cdots$ & 4,300 & $\cdots$ & $<1$ & $\cdots$ & 1 \\
\hline $08-20-88$ & 5,900 & - & 4,200 & --- & 1,600 & --. & $<1$ & -- & 2 \\
\hline $07-07-90$ & 2,300 & - & 5,200 & --- & 2,300 & --- & $<1$ & - & 4 \\
\hline $07-20-90$ & 4,800 & 21 & 2,300 & 5 & 1,000 & $<1$ & $<2$ & 1 & 2 \\
\hline $07-24-90$ & 1,360 & -.. & 2,400 & -- & 970 & $\ldots$ & 6 & -- & 2 \\
\hline $07-24-90$ & 5,260 & 36 & 4,600 & 49 & 1,400 & $<1$ & $<3$ & $<1$ & $<1$ \\
\hline $01-05-91$ & 1,050 & -- & 800 & --- & 450 & -- & $<1$ & - & 5 \\
\hline $02-09-92$ & 95 & 8 & $<100$ & $<3$ & 40 & $<1$ & $<1$ & $<1$ & $<1$ \\
\hline $02-13-92$ & 4,500 & 14 & 900 & 8 & 1,000 & $<1$ & $<1$ & $<1$ & 2 \\
\hline $02-13-92$ & 4,300 & 11 & 500 & 5 & 270 & $<1$ & $<1$ & $<1$ & 1 \\
\hline
\end{tabular}


Table 8. Analytical results of surface-water samples, Rillito Creek basin Trace elements-Continued

\begin{tabular}{|c|c|c|c|c|c|c|c|c|}
\hline Date & $\begin{array}{c}\text { Arsenic, } \\
\text { dissolved } \\
\text { ( } \mu \mathrm{g} / \mathrm{L} \text { as As) }\end{array}$ & $\begin{array}{c}\text { Arsenic, } \\
\text { total } \\
(\mu \mathrm{g} / \mathrm{L} \text { as As })\end{array}$ & $\begin{array}{c}\text { Copper, } \\
\text { dissolved } \\
(\mu \mathrm{g} / \mathrm{L} \text { as } \mathrm{Cu})\end{array}$ & $\begin{array}{c}\text { Copper, } \\
\text { total } \\
(\mu \mathrm{g} / \mathrm{L} \text { as } \mathrm{Cu})\end{array}$ & $\begin{array}{c}\text { Mercury, } \\
\text { dissolved } \\
\text { ( } \mu \mathrm{g} / \mathrm{L} \text { as Hg) }\end{array}$ & $\begin{array}{c}\text { Mercury, } \\
\text { total } \\
(\mu \mathrm{g} / \mathrm{L} \text { as } \mathrm{Hg})\end{array}$ & $\begin{array}{l}\text { Chromium, } \\
\text { dissolved } \\
\text { ( } \mu \mathrm{g} / \mathrm{L} \text { as } \mathrm{Cr} \text { ) }\end{array}$ & $\begin{array}{c}\text { Chromium, } \\
\text { total } \\
\text { ( } \mu \mathrm{g} / \mathrm{L} \text { as } \mathrm{Cr} \text { ) }\end{array}$ \\
\hline \multicolumn{9}{|c|}{ Tanque Verde Creek at Tieson } \\
\hline $08-25-87$ & -- & $<1$ & - & 7 & $\cdots$ & $<0.10$ & -- & 130 \\
\hline $02-02-88$ & $<1$ & $<1$ & 6 & $<0.1$ & $<0.10$ & $<1$ & 1 & $\cdots$ \\
\hline $08-20-88$ & --- & 8 & - & 340 & -- & $<.10$ & -- & 120 \\
\hline 08-03-89 & --- & --- & -- & 310 & - & -- & -- & 68 \\
\hline $08-18-89$ & -.- & 17 & -.. & 1,200 & -- & 1.0 & --- & 350 \\
\hline $07-07-90$ & --- & 6 & --- & 390 & --- & .50 & -- & 110 \\
\hline $07-08-90$ & --- & 10 & --- & 450 & --- & .50 & -- & 170 \\
\hline $07-20-90$ & 5 & 7 & 20 & 260 & $<.1$ & .30 & 2 & 99 \\
\hline $07-24-90$ & --- & 4 & -- & 320 & - & .30 & - & 97 \\
\hline $08-03-90$ & $\ldots$ & 13 & - & 390 & $\cdots$ & .50 & - & 140 \\
\hline $08-14-90$ & -- & 27 & --- & 660 & -- & 2.8 & -- & 210 \\
\hline $12-28-90$ & -.- & 15 & -- & 400 & $\cdots$ & .30 & -.. & 120 \\
\hline $01-05-91$ & --. & 5 &.- & 180 & 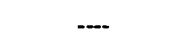 & .30 & -.. & 55 \\
\hline $01-06-92$ & $<1$ & $<1$ & 10 & -- & $<.1$ & $<.10$ & 3 & 3 \\
\hline $02-09-92$ & $<1$ & $<1$ & $<10$ & 6 & $<.1$ & $<.10$ & $<1$ & 2 \\
\hline $02-21-92$ & $<1$ & $<1$ & $<10$ & 2 & $<.1$ & $<.10$ & $<1$ & $<1$ \\
\hline \multicolumn{9}{|c|}{ Pantano Wash at Broad way Boulevard } \\
\hline $11-01-87$ & 3 & 5 & $<10$ & 140 & $\ldots$ & 1.1 & $<10$ & 48 \\
\hline $07-30-88$ & -- & 10 & -- & 520 & 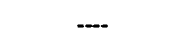 & $\cdots$ & - & 160 \\
\hline $07-24-90$ & 6 & 14 & 20 & 510 & $\cdots$ & .50 & $<1$ & 160 \\
\hline $07-24-90$ & 4 & 17 & 30 & 600 & $<.1$ & .70 & $<1$ & 200 \\
\hline 08-03-90 & --- & 21 & - & 630 & -- & .90 & --- & 230 \\
\hline $02-13-92$ & 2 & 3 & $<10$ & 130 & $<.1$ & $<.10$ & $<1$ & 39 \\
\hline $12-29-92$ & 2 & -- & $<10$ & --- & $<.1$ & -- & $<1$ & -- \\
\hline \multicolumn{9}{|c|}{ Alamo Wash at Glenn Street } \\
\hline $02-25-87$ & -- & 3 & -- & 21 & $\cdots$ & $<.10$ & -- & 20 \\
\hline $08-11-87$ & 13 & --- & 10 & --- & .2 & -- & $<10$ & -- \\
\hline $09-04-87$ & 4 & 5 & 30 & 42 & $<.1$ & .10 & 20 & 20 \\
\hline $08-20-88$ & 11 & 12 & 340 & 340 & $<.1$ & $<.10$ & 1 & 120 \\
\hline $07-20-90$ & 9 & 12 & 10 & 140 & $<.1$ & .90 & 3 & 63 \\
\hline $07-24-90$ & -- & 9 & --- & 84 & --- & .20 & -- & 37 \\
\hline $09-14-90$ & -- & 38 & -- & 200 & -- & .20 & -- & 34 \\
\hline $10-27-91$ & 3 & 3 & 20 & 40 & $<1$ & .20 & 2 & 23 \\
\hline $11-15-91$ & 2 & 2 & 20 & 94 & $<.1$ & $<.10$ & $<1$ & 5 \\
\hline $12-11-91$ & 2 & 5 & $<10$ & -- & $<.1$ & $<.10$ & $<1$ & 5 \\
\hline $01-06-92$ & $<1$ & 3 & 10 & --- & $<.1$ & $<.10$ & 2 & 14 \\
\hline \multicolumn{9}{|c|}{ Rillito Creek at Dodge Boulevard } \\
\hline $07-30-88$ & $\cdots$ & 8 & - & 2,500 & - & $\cdots$ & - & 300 \\
\hline $08-20-88$ & --- & 10 & -- & 630 & --- & .20 & -- & 200 \\
\hline $07-07-90$ &.- & 15 & - & 960 & $\ldots$ & 1.0 & - & 240 \\
\hline $07-20-90$ & 10 & 15 & 20 & 260 & $<.1$ & 1.2 & $<1$ & 130 \\
\hline $07-24-90$ & $\ldots$ & 10 & -- & 430 & -- & .50 & -- & 160 \\
\hline $07-24-90$ & 5 & 18 & 30 & 580 & $<.1$ & .70 & 4 & 190 \\
\hline $01-05-91$ & --- & 24 &.- & 160 & --- & .30 & -- & 57 \\
\hline $02-09-92$ & $<1$ & $<1$ & $<10$ & 14 & .1 & .10 & $<1$ & 3 \\
\hline $02-13-92$ & 4 & 4 & $<10$ & 170 & $<.1$ & $<.10$ & $<1$ & 57 \\
\hline $02-13-92$ & 1 & 3 & $<10$ & 120 & $<.1$ & .10 & $<1$ & 33 \\
\hline
\end{tabular}


Table 8. Analytical results of surface-water samples, Rillito Creek basin-Continued Trace elements-Continued

\begin{tabular}{|c|c|c|c|c|c|c|c|c|}
\hline Date & $\begin{array}{c}\text { Boron, } \\
\text { dlssolved } \\
\text { ( } \mu \mathrm{g} / \mathrm{L} \text { as B) }\end{array}$ & $\begin{array}{l}\text { Vandium, } \\
\text { dissolved } \\
\text { ( } \mu \mathrm{g} / \mathrm{L} \text { as V) }\end{array}$ & $\begin{array}{c}\text { Silver, } \\
\text { dissolved } \\
(\mu \mathrm{g} / \mathrm{L} \text { as } \mathrm{Ag})\end{array}$ & $\begin{array}{l}\text { Silver, total } \\
\text { recoverable } \\
\text { ( } \mu \mathrm{g} / \mathrm{L} \text { as } \mathrm{Ag})\end{array}$ & $\begin{array}{l}\text { Cadmium, } \\
\text { dissolved } \\
(\mu \mathrm{g} / \mathrm{L} \text { as Cd })\end{array}$ & $\begin{array}{l}\text { Cadmium, total } \\
\text { recoverable } \\
\text { ( } \mu \mathrm{g} / \mathrm{L} \text { as Cd) }\end{array}$ & $\begin{array}{c}\text { Lead, } \\
\text { dissolved } \\
(\mu \mathrm{g} / \mathrm{L} \text { as Pb) }\end{array}$ & $\begin{array}{l}\text { Lead, total } \\
\text { recoverable } \\
(\mu \mathrm{g} / \mathrm{L} \text { as Pb) }\end{array}$ \\
\hline \multicolumn{9}{|c|}{ Tanque Verde Creek at Tucson } \\
\hline $08-25-87$ & -- & - & --- & $<1$ & $\ldots$ & $<1$ & - & $<5$ \\
\hline $02-02-88$ & $<10$ & 2 & $<1.0$ & $<1$ & 1.0 & $<1$ & $<$ & $<5$ \\
\hline $08-20-88$ & -- & - & - & $<1$ & -- & 1 & - & 300 \\
\hline 08-03-89 & - & - & - & 1 & --- & 4 & - & 400 \\
\hline $08-18-89$ & -- & -- & -- & 2 & --- & 12 & - & 1,100 \\
\hline $07-07-90$ & - & $\ldots$ & --- & 1 & $\cdots$ & 3 & - & 210 \\
\hline $07-08-90$ & - & - & --- & 1 & $\ldots$ & 4 & - & 240 \\
\hline $07-20-90$ & 30 & 12 & $<1.0$ & $<1$ & $<1.0$ & 2 & 1 & 200 \\
\hline $07-24-90$ & -- & -- & - & 1 & -- & 2 & - & 260 \\
\hline $08-03-90$ & -- & -- & - & 1 & --- & 4 & - & 310 \\
\hline $08-14-90$ & -- & -- & -- & 1 & --- & 4 & - & 520 \\
\hline $12-28-90$ & -- & - & - & 1 & --- & 3 & - & 300 \\
\hline $01-05-91$ & -- & - & -- & $<1$ & --- & 3 & - & 150 \\
\hline $01-06-92$ & $<10$ & 4 & $<1.0$ & $<1$ & $<1.0$ & $<1$ & 1 & 11 \\
\hline $02-09-92$ & $<10$ & 4 & $<1.0$ & $<1$ & $<1.0$ & $<1$ & $<1$ & 2 \\
\hline $02-21-92$ & $<10$ & -- & $<1.0$ & $<1$ & $<1.0$ & $<1$ & $<1$ & $<1$ \\
\hline \multicolumn{9}{|c|}{ Pantano Wash at Broadway Boulevard } \\
\hline $11-01-87$ & 20 & 11 & 1.0 & 1 & $<1.0$ & 1 & 5 & 100 \\
\hline $07-30-88$ & - & -- & - & $<1$ & --- & $<1$ & $\cdots$ & 500 \\
\hline $07-24-90$ & 30 & 10 & 1.0 & 1 & $<1.0$ & $<1$ & $<1$ & 400 \\
\hline $07-24-90$ & 30 & 12 & 1.0 & 2 & $<1.0$ & $<1$ & 3 & 21 \\
\hline $08-03-90$ & - & - & -- & 2 & --- & 1 & $-\cdots$ & 450 \\
\hline $02-13-92$ & 20 & 3 & $<1.0$ & $<1$ & $<1.0$ & 2 & 1 & 130 \\
\hline $12-29-92$ & 10 & 6 & $<1.0$ & --- & $<1.0$ & $\cdots$ & 1 & --- \\
\hline \multicolumn{9}{|c|}{ Alamo Wash at Glenn Street } \\
\hline $02-25-87$ & -- & $\cdots$ & -- & $<1$ & --- & $<1$ & $\cdots$ & 60 \\
\hline $08-11-87$ & 40 & 11 & $<1.0$ & $\cdots$ & $<1.0$ & $\ldots$ & $<5$ & $\cdots$ \\
\hline $09-04-87$ & 30 & 9 & $<1.0$ & 1 & 1.0 & 1 & $<$ & 77 \\
\hline $08-20-88$ & 20 & 13 & $<1.0$ & 2 & 2.0 & 2 & $\infty$ & -- \\
\hline $07-20-90$ & 30 & 10 & $<1.0$ & $<1$ & $<1.0$ & 4 & 1 & 210 \\
\hline $07-24-90$ & -- & - & - & $<1$ & $\cdots$ & 2 & - & 110 \\
\hline $09-14-90$ & -- & -- & -- & $<1$ & --- & 4 & - & 200 \\
\hline $10-27-91$ & 20 & 12 & $<1.0$ & $<1$ & $<1.0$ & 1 & 1 & 13 \\
\hline $11-15-91$ & 20 & 5 & $<1.0$ & $<1$ & $<1.0$ & $<1$ & 2 & 28 \\
\hline $12-11-91$ & 20 & 4 & $<1.0$ & $<1$ & $<1.0$ & $<1$ & $<1$ & 30 \\
\hline 01-06-92 & 10 & 4 & $<1.0$ & $<1$ & $<1.0$ & 2 & 2 & 110 \\
\hline \multicolumn{9}{|c|}{ Rillito Creek at Dodge Boulevard } \\
\hline $07-30-88$ & - & - & -- & 4 & --- & 1 & - & 1,900 \\
\hline $08-20-88$ & - & - & - & 1 & 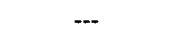 & 6 & - & -- \\
\hline $07-07-90$ & - & -- & - & 2 & $\cdots$ & 3 & -- & 600 \\
\hline $07-20-90$ & 40 & 13 & $<1.0$ & 1 & $<1.0$ & 4 & 1 & 370 \\
\hline $07-24-90$ & - & -- & - & 1 & $\cdots$ & 2 & -- & 320 \\
\hline $07-24-90$ & 40 & 18 & $<1.0$ & 2 & $<1.0$ & $<1$ & 2 & 17 \\
\hline $01-05-91$ & - & - & $\cdots$ & $<1$ & -- & 3 & -- & 160 \\
\hline $02-09-92$ & $<10$ & 5 & $<1.0$ & $<1$ & $<1.0$ & $<1$ & $<1$ & 7 \\
\hline $02-13-92$ & 20 & 7 & $<1.0$ & $<1$ & $<1.0$ & 2 & $<1$ & 120 \\
\hline $02-13-92$ & 10 & 6 & $<1.0$ & $<1$ & $<1.0$ & 1 & 2 & 100 \\
\hline
\end{tabular}


Table 8. Analytical results of surface-water samples, Rillito Creek basin-Continued Trace elements-Continued

\begin{tabular}{|c|c|c|c|c|c|c|c|c|}
\hline Date & $\begin{array}{c}\text { Nickel, } \\
\text { dlssolved } \\
(\mu \mathrm{g} / \mathrm{L} \text { as Ni) }\end{array}$ & $\begin{array}{c}\text { Nickel, } \\
\text { total } \\
\text { recoverable } \\
(\mu \mathrm{g} / \mathrm{L} \text { as } \mathrm{Ni})\end{array}$ & $\begin{array}{c}\text { Manganese, } \\
\text { total } \\
\text { recoverable } \\
\text { ( } \mu \text { g/L as Mn) }\end{array}$ & $\begin{array}{l}\text { Iron, total } \\
\text { recoverable } \\
\text { ( } \mu \mathrm{g} / \mathrm{L} \text { as Fe) }\end{array}$ & $\begin{array}{c}\text { Beryllium, } \\
\text { total } \\
\text { recoverable } \\
(\mu g / L \text { as } B e)\end{array}$ & $\begin{array}{c}\text { Lithium, } \\
\text { total } \\
\text { recoverable } \\
\text { ( } \mu \text { g/L as Li) }\end{array}$ & $\begin{array}{c}\text { Aluminum, } \\
\text { total } \\
\text { recoverable } \\
\text { ( } \mu \text { g/L as } \mathrm{Al})\end{array}$ & $\begin{array}{l}\text { Cobalt, total } \\
\text { recoverable } \\
\text { ( } \mu \mathrm{g} / \mathrm{L} \text { as Co) }\end{array}$ \\
\hline \multicolumn{9}{|c|}{ Tanque Verde Creek at Tucson } \\
\hline $08-25-87$ & --- & $<1$ & 40 & 960 & $<10$ & $<10$ & 1,100 & $<1$ \\
\hline $02-02-88$ & 2 & 2 & 20 & 330 & $<10$ & $<10$ & 470 & 3 \\
\hline 08-20-88 & - & 97 & 6,900 & 100,000 & 10 & 220 & 150,000 & 30 \\
\hline 08-03-89 & - & 86 & 6,400 & 85,000 & 10 & 120 & 95,000 & 40 \\
\hline $08-18-89$ & -- & 400 & 21,000 & 510,000 & 20 & 380 & 410,000 & 50 \\
\hline $07-07-90$ & - & 110 & 8,700 & 160,000 & $<10$ & 190 & 150,000 & 70 \\
\hline $07-08-90$ &.- & 130 & 9,000 & 200,000 & $<10$ & 280 & 210,000 & 90 \\
\hline $07-20-90$ & 4 & 100 & 5,100 & 130,000 & 10 & 170 & 130,000 & 40 \\
\hline $07-24-90$ & - & 100 & 6,000 & 130,000 & $<10$ & 160 & 120,000 & 50 \\
\hline 08-03-90 & - & 160 & 6,600 & 170,000 & 10 & 270 & 200,000 & 90 \\
\hline $08-14-90$ & -- & 240 & 12,000 & 270,000 & 10 & 340 & 270,000 & 30 \\
\hline $12-28-90$ & - & 130 & 7,600 & 170,000 & $<10$ & 200 & 71,000 & 80 \\
\hline $01-05-91$ & - & 260 & 3,000 & 72,000 & $<10$ & 110 & 58,000 & 30 \\
\hline $01-06-92$ & $<1$ & 2 & 120 & 3,500 & $<10$ & $<10$ & 4,700 & 3 \\
\hline $02-09-92$ & $<1$ & 1 & 30 & 720 & $<10$ & $<10$ & 1,100 & 2 \\
\hline $02-21-92$ & $<1$ & 3 & 20 & 360 & $<10$ & $<10$ & 830 & $<1$ \\
\hline \multicolumn{9}{|c|}{ Pantano Wash at Broadway Boulevard } \\
\hline $11-01-87$ & $<1$ & 33 & 2,400 & 51,000 & $<10$ & 110 & 56,000 & 20 \\
\hline $07-30-88$ & - & 200 & 15,000 & 170,000 & 20 & 610 & 280,000 & 20 \\
\hline $07-24-90$ & 1 & 190 & 11,000 & 200,000 & 10 & 350 & 220,000 & 100 \\
\hline $07-24-90$ & 3 & 200 & 13,000 & 250,000 & 20 & 450 & 300,000 & 130 \\
\hline 08-03-90 & -- & 300 & 17,000 & 290,000 & 20 & 520 & 320,000 & 170 \\
\hline 02-13-92 & $<1$ & 37 & 2,000 & 42,000 & $<10$ & 70 & 49,000 & 20 \\
\hline $12-29-92$ & $<1$ & $\ldots$ & $\ldots$ & - & $\cdots$ & -- & -- & - \\
\hline \multicolumn{9}{|c|}{ Alamo Wash at Glenn Street } \\
\hline $02-25-87$ & -- & 6 & 150 & 4,500 & $<10$ & $<10$ & 7,300 & 2 \\
\hline $08-11-87$ & 4 & --- & $\cdots$ & - & --- &.-- & - & -- \\
\hline $09-04-87$ & 3 & 7 & 420 & 9,700 & $<10$ & 20 & 17,000 & 5 \\
\hline $08-20-88$ & 1 & 100 & 4,900 & 93,000 & 10 & 180 & 140,000 & 30 \\
\hline $07-20-90$ & 4 & 56 & 1,900 & 59,000 & $<10$ & 90 & 84,000 & 20 \\
\hline $07-24-90$ & -- & 32 & 1,000 & 32,000 & $<10$ & 40 & 43,000 & 10 \\
\hline $09-14-90$ & - & 33 & 1,100 & 28,000 & $<10$ & 40 & 40,000 & 10 \\
\hline $10-27-91$ & 3 & 20 & 320 & 8,500 & $<10$ & 20 & 10,000 & 5 \\
\hline $11-15-91$ & 1 & 5 & 110 & 2,600 & $<10$ & $<10$ & 3,500 & 2 \\
\hline $12-11-91$ & $<1$ & 5 & 140 & 4,300 & $<10$ & 10 & 6,600 & 3 \\
\hline $01-06-92$ & $<1$ & 14 & 340 & 11,000 & $<10$ & 10 & 18,000 & 6 \\
\hline \multicolumn{9}{|c|}{ Rillito Creek at Dodge Boulevard } \\
\hline $07-30-88$ & - & 700 & 49,000 & 340,000 & 60 & 1,600 & 550,000 & 100 \\
\hline $08-20-88$ & -- & 300 & 13,000 & 160,000 & 20 & 390 & 260,000 & 50 \\
\hline 07-07-90 & -- & 270 & 23,000 & 310,000 & $<10$ & 360 & 290,000 & 180 \\
\hline $07-20-90$ & 1 & 170 & 7,300 & 160,000 & 10 & 260 & 180,000 & 50 \\
\hline $07-24-90$ & -- & 160 & 7,000 & 150,000 & $<10$ & 240 & 170,000 & 80 \\
\hline $07-24-90$ & 2 & 240 & 14,000 & 280,000 & 20 & 480 & 280,000 & 150 \\
\hline $01-05-91$ & -- & 64 & 3,300 & 71,000 & $<10$ & 110 & 56,000 & 30 \\
\hline $02-09-92$ & $<1$ & 4 & 120 & 3,600 & $<10$ & $<10$ & 4,400 & 2 \\
\hline $02-13-92$ & 1 & 55 & 3,000 & 73,000 & $<10$ & 110 & 71,000 & 40 \\
\hline $02-13-92$ & 1 & 32 & 1,800 & 44,000 & $<10$ & 70 & 44,000 & 20 \\
\hline
\end{tabular}


Table 8. Analytical results of surface-water samples, Rillito Creek basin Radionuclides

\begin{tabular}{|c|c|c|c|c|c|c|c|}
\hline Date & $\begin{array}{l}\text { Discharge, } \\
\text { Instan- } \\
\text { taneous, } \\
\left(\mathrm{ft}^{3} / \mathrm{s}\right)\end{array}$ & $\begin{array}{c}\text { Gross aipha, } \\
\text { dissoived } \\
\text { ( } \mu \text { g/L } \\
\text { as U-Nat) }\end{array}$ & $\begin{array}{c}\text { Gross aipha, } \\
\text { suspended } \\
\text { ( } \mu \text { g/L } \\
\text { as U-Nat) }\end{array}$ & $\begin{array}{l}\text { Gross beta, } \\
\text { dissoived } \\
\text { (pCl/L as } \\
\text { Sr-90/Y-90) }\end{array}$ & $\begin{array}{c}\text { Gross beta, } \\
\text { suspended } \\
\text { totai } \\
\text { (pCi/L as } \\
\text { Sr-90/Y-90) }\end{array}$ & $\begin{array}{l}\text { Gross beta, } \\
\text { dissoived } \\
\text { (pCi/L as } \\
\text { Cs-137) }\end{array}$ & $\begin{array}{c}\text { Gross beta, } \\
\text { suspended } \\
\text { totai } \\
\text { (pCi/L as } \\
\text { Cs-137) }\end{array}$ \\
\hline \multicolumn{8}{|c|}{ Tanque Verde Creek at Tucson } \\
\hline $02-02-88$ & 44 & 0.6 & 0.4 & 0.9 & $<0.4$ & 1.0 & $<0.4$ \\
\hline $08-01-89$ & 6 & .8 & $<.6$ & 4.8 & $<6$ & 6.1 & .6 \\
\hline 08-03-89 & 69 & .7 & 120 & 11 & 180 & 12 & 220 \\
\hline $07-07-90$ & 450 & 3.4 & 460 & 13 & 320 & 17 & 340 \\
\hline $07-08-90$ & 1,600 & .7 & 150 & 9.9 & 160 & 13 & 190 \\
\hline $01-06-92$ & 105 & $<.6$ & 2.2 & 1.4 & 4 & 1.5 & 4.4 \\
\hline \multicolumn{8}{|c|}{ Pantano Wash at Broadway Boulevard } \\
\hline $11-01-87$ & 50 & .7 & 180 & 2.8 & 97 & 3.5 & 110 \\
\hline $07-20-88$ & 20 & 1.5 & 400 & 5.6 & 220 & 7.7 & 250 \\
\hline $07-24-90$ & 4,470 & 4.4 & 93 & 5.2 & 23 & 7.0 & 25 \\
\hline $08-03-90$ & 5,300 & 1.4 & 1,500 & 4.6 & 910 & 6.2 & 1,000 \\
\hline $02-13-92$ & 97 & .8 & 340 & 2.0 & 120 & 2.4 & 130 \\
\hline \multicolumn{8}{|c|}{ Alamo Wash at Glenn Streel } \\
\hline $09-04-87$ & 58 & $<.4$ & 7.0 & 1.9 & 7.7 & 2.3 & 9.0 \\
\hline $10-14-88$ & 50 & .6 & 1.2 & 3.1 & 110 & 4.1 & 120 \\
\hline $11-15-91$ & 33 & $<.6$ & 10 & 1.7 & 6.8 & 1.9 & 7.5 \\
\hline \multicolumn{8}{|c|}{ Rillito Creek at Dodge Boulerard } \\
\hline $10-20-88$ & 265 & 1.2 & 27 & 2.7 & 60 & 3.5 & 69 \\
\hline $07-24-90$ & 5,260 & 4.0 & 710 & 6.1 & 360 & 8.2 & 390 \\
\hline $02-09-92$ & 95 & .8 & 7.5 & .9 & 5.2 & 1.0 & 5.7 \\
\hline
\end{tabular}


Table 8. Analytical results of surface-water samples, Rillito Creek basin Organochlorine pesticides

[DDD, dichlorodiphenyldichloroethane; DDE, dichlorodiphenylethylene; DDT, dichlorodiphenyltrichloroethane; PCB, polychlorinated biphenyl]

\begin{tabular}{|c|c|c|c|c|c|c|c|c|c|}
\hline Date & $\begin{array}{c}\text { Dis- } \\
\text { charge, } \\
\text { instan- } \\
\text { taneous, } \\
\left(\mathrm{ft}^{3} / \mathrm{s}\right)\end{array}$ & $\begin{array}{c}\text { Per- } \\
\text { thane, } \\
\text { total } \\
(\mu \mathrm{g} / \mathrm{L})\end{array}$ & $\begin{array}{c}\text { Endo- } \\
\text { sulfate, } \\
\text { total } \\
(\mu \mathrm{g} / L)\end{array}$ & $\begin{array}{c}\text { Aldrín, } \\
\text { total } \\
(\mu g / L)\end{array}$ & $\begin{array}{l}\text { Chlor- } \\
\text { dane, } \\
\text { total } \\
(\mu g / L)\end{array}$ & $\begin{array}{c}\text { DDD, } \\
\text { total } \\
(\mu \mathrm{g} / \mathrm{L})\end{array}$ & $\begin{array}{c}\text { DDE, } \\
\text { total } \\
(\mu g / L)\end{array}$ & $\begin{array}{c}\text { DDT, } \\
\text { total } \\
(\mu \mathrm{g} / \mathrm{L})\end{array}$ & $\begin{array}{l}\text { Diel- } \\
\text { drin, } \\
\text { total } \\
(\mu \mathrm{g} / \mathrm{L})\end{array}$ \\
\hline \multicolumn{10}{|c|}{ Tanque Verde Creek at Tucson } \\
\hline $02-02-88$ & 44 & $<0.1$ & $<0.01$ & $<0.01$ & $<0.1$ & $<0.01$ & $<0.01$ & $<0.01$ & $<0.01$ \\
\hline $07-24-90$ & 4,400 & $<1$ & $<.01$ & $<.01$ & .2 & $<01$ & $<01$ & $<.01$ & $<.01$ \\
\hline 01-06-92 & 105 & $<1$ & $<.01$ & $<01$ & $<1$ & $<.01$ & $<01$ & $<.01$ & $<.01$ \\
\hline $02-09-92$ & 120 & $<1$ & $<.01$ & $<.01$ & $<1$ & $<.01$ & $<.01$ & $<.01$ & $<.01$ \\
\hline \multicolumn{10}{|c|}{ Pantano Wash at Broadway Bouleyard } \\
\hline $11-01-87$ & 50 & $<1$ & $<.01$ & $<.01$ & .2 & $<.01$ & $<.01$ & $<.01$ & $<.01$ \\
\hline $07-24-90$ & & $<1$ & $<.01$ & $<.01$ & .1 & $<.01$ & $<.01$ & $<.01$ & $<.01$ \\
\hline $02-13-92$ & 97 & $<1$ & $<.01$ & $<01$ & $<1$ & $<01$ & $<.01$ & $<.01$ & $<.01$ \\
\hline \multicolumn{10}{|c|}{ Alamo Wash at Glemn Street } \\
\hline $07-26-87$ & 415 & $<1$ & $<.01$ & .02 & 1.5 & .03 & .05 & $<.01$ & .19 \\
\hline 09-04-87 & 58 & $<1$ & $<.01$ & $<01$ & .2 & $<01$ & $<.01$ & $<.01$ & .01 \\
\hline $07-24-90$ & 870 & $<.1$ & $<.01$ & $<01$ & .6 & $<.01$ & $<.01$ & $<.01$ & $<.01$ \\
\hline $09-14-90$ & 695 & $<1$ & $<.01$ & $<01$ & 1.1 & $<10$ & $<10$ & $<.10$ & $<.15$ \\
\hline $10-27-91$ & 62 & $<1$ & $<.01$ & $<01$ & .1 & $<.01$ & $<.01$ & $<.01$ & .01 \\
\hline $11-15-91$ & 33 & $<1$ & $<.01$ & $<01$ & .1 & $<01$ & $<.01$ & $<.01$ & .01 \\
\hline $12-11-91$ & 49 & $<1$ & $<.01$ & $<01$ & .1 & $<01$ & .01 & .01 & .01 \\
\hline $01-06-92$ & 325 & $<.1$ & $<.01$ & $<01$ & $<1$ & $<.01$ & .01 & $<.01$ & .02 \\
\hline \multicolumn{10}{|c|}{ Rilito Creek at Dodge Boulevard } \\
\hline $07-30-88$ & 5,300 & $<.1$ & $<.01$ & $<.01$ & .1 & $<.01$ & $<.01$ & $<.01$ & $<.01$ \\
\hline $07-24-90$ & 1,360 & $<1$ & $<.01$ & $<01$ & .1 & $<.01$ & $<.01$ & $<.01$ & .02 \\
\hline $02-09-92$ & 95 & $<1$ & $<.01$ & $<01$ & $<1$ & $<.01$ & $<.01$ & $<.01$ & $<.01$ \\
\hline $02-13-92$ & 4,500 & $<1$ & $<.01$ & $<01$ & $<1$ & $<01$ & $<.01$ & $<.01$ & $<.01$ \\
\hline
\end{tabular}


Table 8. Analytical results of surface-water samples, Rillito Creek basin-Continued Organochlorine pesticides-Continued

\begin{tabular}{|c|c|c|c|c|c|c|c|c|c|}
\hline Date & $\begin{array}{c}\text { Endrin, } \\
\text { total } \\
(\mu \mathrm{g} / \mathrm{L})\end{array}$ & $\begin{array}{c}\text { Hepta- } \\
\text { chlor, } \\
\text { total } \\
(\mu \mathrm{g} / \mathrm{L})\end{array}$ & $\begin{array}{l}\text { Hepta- } \\
\text { chlor } \\
\text { epoxide, } \\
\text { total } \\
(\mu g / L)\end{array}$ & $\begin{array}{c}\text { Lindane, } \\
\text { total } \\
(\mu \mathrm{g} / \mathrm{L})\end{array}$ & $\begin{array}{c}\text { Toxa- } \\
\text { phene, } \\
\text { totai } \\
(\mu g / L)\end{array}$ & $\begin{array}{c}\text { PCB, } \\
\text { total } \\
(\mu g / L)\end{array}$ & $\begin{array}{c}\text { Naph- } \\
\text { thaiene, } \\
\text { poly- } \\
\text { chlor } \\
\text { total } \\
(\mu g / L)\end{array}$ & $\begin{array}{l}\text { Meth- } \\
\text { oxy- } \\
\text { chlor, } \\
\text { total } \\
(\mu \mathrm{g} / \mathrm{L})\end{array}$ & $\begin{array}{c}\text { Mirex, } \\
\text { totai } \\
(\mu g / L)\end{array}$ \\
\hline \multicolumn{10}{|c|}{ Tanque verde Creek at Tucson } \\
\hline $02-02-88$ & $<0.01$ & $<0.01$ & $<0.01$ & $<0.01$ & $<1$ & $<0.1$ & $<0.10$ & $<0.01$ & $<0.01$ \\
\hline $07-24-90$ & $<01$ & $<.01$ & $<01$ & $<.01$ & $<1$ & $<1$ & $<.10$ & $<01$ & $<.01$ \\
\hline $01-06-92$ & $<01$ & $<.01$ & $<01$ & $<.01$ & $<1$ & $<1$ & $<.10$ & $<.01$ & $<.01$ \\
\hline $02-09-92$ & $<01$ & $<.01$ & $<.01$ & $<.01$ & $<1$ & $<1$ & $<.10$ & $<01$ & $<.01$ \\
\hline \multicolumn{10}{|c|}{ Pantano Wash at Broadway Boulevard } \\
\hline $11-01-87$ & $<.01$ & $<.01$ & $<01$ & $<0.01$ & $<1$ & $<1$ & $<.10$ & $<01$ & $<.01$ \\
\hline $07-24-90$ & $<.01$ & $<.01$ & $<01$ & $<.01$ & $<1$ & $<1$ & $<.10$ & $<01$ & $<.01$ \\
\hline $02-13-92$ & $<.01$ & $<.01$ & $<.01$ & $<.01$ & $<1$ & $<1$ & $<.10$ & $<.01$ & $<.01$ \\
\hline \multicolumn{10}{|c|}{ Alamo Wash at Glenn Street } \\
\hline $07-26-87$ & $<01$ & $<.01$ & $<01$ & $<.01$ & $<1$ & $<1$ & $<.10$ & $<01$ & $<.01$ \\
\hline $09-04-87$ & $<01$ & $<.01$ & $<01$ & $<.01$ & $<1$ & $<1$ & $<.10$ & $<01$ & $<.01$ \\
\hline $07-24-90$ & .01 & $<.01$ & $<.02$ & $<.01$ & $<1$ & $<1$ & $<.10$ & $<01$ & $<.01$ \\
\hline $09-14-90$ & $<.01$ & $<.01$ & $<01$ & $<.01$ & $<1$ & $<1$ & $<.10$ & $<01$ & $<.01$ \\
\hline $10-27-91$ & $<.01$ & $<.01$ & $<.01$ & $<.01$ & $<1$ & $<1$ & $<.10$ & $<01$ & $<.01$ \\
\hline $11-15-91$ & $<.01$ & $<.01$ & $<01$ & $<.01$ & $<1$ & $<1$ & $<.10$ & $<.01$ & $<.01$ \\
\hline $12-11-91$ & $<01$ & $<.01$ & $<01$ & $<.01$ & $<1$ & $<1$ & $<.10$ & $<01$ & $<.01$ \\
\hline $01-06-92$ & $<.01$ & $<.01$ & $<01$ & $<.01$ & $<1$ & $<1$ & $<.10$ & $<.01$ & $<.01$ \\
\hline \multicolumn{10}{|c|}{ Rillito Creek at Dodge Boulevard } \\
\hline $07-30-88$ & $<.01$ & $<.01$ & $<01$ & $<.01$ & $<1$ & $<1$ & $<.10$ & $<.01$ & $<.01$ \\
\hline $07-24-90$ & $<.01$ & $<.01$ & $<01$ & $<.01$ & $<1$ & $<1$ & $<.10$ & $<01$ & $<.01$ \\
\hline $02-09-92$ & $<.01$ & $<.01$ & $<01$ & $<.01$ & $<1$ & $<.1$ & $<.10$ & $<.01$ & $<.01$ \\
\hline $02-13-92$ & $<.01$ & $<.01$ & $<01$ & $<.01$ & $<1$ & $<1$ & $<.10$ & $<01$ & $<.01$ \\
\hline
\end{tabular}


Table 8. Analytical results of surface-water samples, Rillito Creek basin Priority pollutants

\begin{tabular}{|c|c|c|c|c|c|c|c|c|c|c|}
\hline Date & $\begin{array}{c}\text { Dis- } \\
\text { charge, } \\
\text { instan- } \\
\text { taneous, } \\
\left(\mathrm{ft}^{3} / 8\right)\end{array}$ & $\begin{array}{c}\text { Para- } \\
\text { chloro- } \\
\text { meta } \\
\text { cresol, } \\
\text { total } \\
(\mu \mathrm{g} / \mathrm{L})\end{array}$ & $\begin{array}{c}\text { 2-Chioro- } \\
\text { phenol, } \\
\text { total } \\
(\mu \mathrm{g} / \mathrm{L})\end{array}$ & $\begin{array}{c}\text { 2,4-Di- } \\
\text { chloro- } \\
\text { phenol, } \\
\text { total } \\
(\mu \mathrm{g} / \mathrm{L})\end{array}$ & $\begin{array}{c}\text { 2,4,6-Tri- } \\
\text { chloro- } \\
\text { phenol, } \\
\text { total } \\
(\mu \mathrm{g} / \mathrm{L})\end{array}$ & $\begin{array}{c}\text { 2,4-Di- } \\
\text { methyl- } \\
\text { phenol, } \\
\text { total } \\
(\mu \mathrm{g} / \mathrm{L})\end{array}$ & $\begin{array}{c}\text { 4,6- } \\
\text { Dinitro- } \\
\text { ortho- } \\
\text { cresol, } \\
\text { total } \\
(\mu \mathrm{g} / \mathrm{L})\end{array}$ & $\begin{array}{c}\text { 2,4-Di- } \\
\text { nitro- } \\
\text { phenol, } \\
\text { total } \\
(\mu \mathrm{g} / \mathrm{L})\end{array}$ & $\begin{array}{c}\text { 2-Nitro- } \\
\text { phenol, } \\
\text { total } \\
(\mu \mathrm{g} / \mathrm{L})\end{array}$ & $\begin{array}{c}\text { 4-Nitro- } \\
\text { phenol, } \\
\text { total } \\
(\mu \mathrm{g} / \mathrm{L})\end{array}$ \\
\hline \multicolumn{11}{|c|}{ Tanque Verde Creek at Turson } \\
\hline $02-02-88$ & 44 & $<30.0$ & $<5.0$ & $<5.0$ & $<20.0$ & $<5.0$ & $<30.0$ & $<20.0$ & $<5.0$ & $<30.0$ \\
\hline $08-20-88$ & 3,800 & $<30.0$ & $<5.0$ & $<5.0$ & $<20.0$ & $<5.0$ & $<30.0$ & $<20.0$ & $<5.0$ & $<30.0$ \\
\hline $07-20-90$ & 2,200 & $<30.0$ & $<5.0$ & $<5.0$ & $<20.0$ & $<5.0$ & $<30.0$ & $<20.0$ & $<5.0$ & $<30.0$ \\
\hline $07-24-90$ & 4,400 & $<30.0$ & $<5.0$ & $<5.0$ & $<20.0$ & $<5.0$ & $<30.0$ & $<20.0$ & $<5.0$ & $<30.0$ \\
\hline $01-05-91$ & 1,080 & $<30.0$ & $<5.0$ & $<5.0$ & $<20.0$ & $<5.0$ & $<30.0$ & $<20.0$ & $<5.0$ & $<30.0$ \\
\hline $01-06-92$ & 105 & $<30.0$ & $<5.0$ & $<5.0$ & $<20.0$ & $<5.0$ & $<30.0$ & $<20.0$ & $<5.0$ & $<30.0$ \\
\hline $02-09-92$ & 120 & $<30.0$ & $<5.0$ & $<5.0$ & $<20.0$ & $<5.0$ & $<30.0$ & $<20.0$ & $<5.0$ & $<30.0$ \\
\hline \multicolumn{11}{|c|}{ Pantano Wash at Broad way Boulevard } \\
\hline $11-01-87$ & 50 & $<30.0$ & $<5.0$ & $<5.0$ & $<20.0$ & $<5.0$ & $<30.0$ & $<20.0$ & $<5.0$ & $<30.0$ \\
\hline $02-13-92$ & 97 & $<30.0$ & $<5.0$ & $<5.0$ & $<20.0$ & $<5.0$ & $<30.0$ & $<20.0$ & $<5.0$ & $<30.0$ \\
\hline \multicolumn{11}{|c|}{ Alamo Wast at Clenn Street } \\
\hline $07-26-87$ & 415 & $<30.0$ & $<5.0$ & $<5.0$ & $<20.0$ & $<5.0$ & $<30.0$ & $<20.0$ & $<5.0$ & $<30.0$ \\
\hline $08-11-87$ & 230 & $<30.0$ & $<5.0$ & $<5.0$ & $<20.0$ & $<5.0$ & $<30.0$ & $<20.0$ & $<5.0$ & $<30.0$ \\
\hline $08-20-88$ & 4,000 & $<30.0$ & $<5.0$ & $<5.0$ & $<20.0$ & $<5.0$ & $<30.0$ & $<20.0$ & $<5.0$ & $<30.0$ \\
\hline $07-20-90$ & 629 & $<30.0$ & $<5.0$ & $<5.0$ & $<20.0$ & $<5.0$ & $<30.0$ & $<20.0$ & $<5.0$ & $<30.0$ \\
\hline $07-24-90$ & 870 & $<30.0$ & $<5.0$ & $<5.0$ & $<20.0$ & $<5.0$ & $<30.0$ & $<20.0$ & $<5.0$ & $<30.0$ \\
\hline $09-14-90$ & 695 & $<30.0$ & $<5.0$ & $<5.0$ & $<20.0$ & $<5.0$ & $<30.0$ & $<20.0$ & $<5.0$ & $<30.0$ \\
\hline $10-27-91$ & 62 & $<30.0$ & $<5.0$ & $<5.0$ & $<20.0$ & $<5.0$ & $<30.0$ & $<20.0$ & $<5.0$ & $<30.0$ \\
\hline $11-15-91$ & 33 & $<30.0$ & $<5.0$ & $<5.0$ & $<20.0$ & $<5.0$ & $<30.0$ & $<20.0$ & $<5.0$ & $<30.0$ \\
\hline $12-11-91$ & 49 & $<30.0$ & $<5.0$ & $<5.0$ & $<20.0$ & $<5.0$ & $<30.0$ & $<20.0$ & $<5.0$ & $<30.0$ \\
\hline $01-06-92$ & 325 & $<30.0$ & $<5.0$ & $<5.0$ & $<20.0$ & $<5.0$ & $<30.0$ & $<20.0$ & $<5.0$ & $<30.0$ \\
\hline \multicolumn{11}{|c|}{ Rilito Creek at Dodge Boulevard } \\
\hline $07-30-88$ & 5,300 & $<30.0$ & $<5.0$ & $<5.0$ & $<20.0$ & $<5.0$ & $<30.0$ & $<20.0$ & $<5.0$ & $<30.0$ \\
\hline $08-20-88$ & 5,900 & $<30.0$ & $<5.0$ & $<5.0$ & $<20.0$ & $<5.0$ & $<30.0$ & $<20.0$ & $<5.0$ & $<30.0$ \\
\hline $07-20-90$ & 4,800 & $<30.0$ & $<5.0$ & $<5.0$ & $<20.0$ & $<5.0$ & $<30.0$ & $<20.0$ & $<5.0$ & $<30.0$ \\
\hline $01-05-91$ & 1,050 & $<30.0$ & $<5.0$ & $<5.0$ & $<20.0$ & $<5.0$ & $<30.0$ & $<20.0$ & $<5.0$ & $<30.0$ \\
\hline $02-09-92$ & 95 & $<30.0$ & $<5.0$ & $<5.0$ & $<20.0$ & $<5.0$ & $<30.0$ & $<20.0$ & $<5.0$ & $<30.0$ \\
\hline $02-13-92$ & 4,500 & $<30.0$ & $<5.0$ & $<5.0$ & $<20.0$ & $<5.0$ & $<30.0$ & $<20.0$ & $<5.0$ & $<30.0$ \\
\hline
\end{tabular}


Table 8. Analytical results of surface-water samples, Rillito Creek basin Priority pollutants-Continued

\begin{tabular}{|c|c|c|c|c|c|c|c|c|c|}
\hline Date & $\begin{array}{l}\text { Penta. } \\
\text { chloro- } \\
\text { phenol, } \\
\text { total } \\
(\mu \mathrm{g} / \mathrm{L})\end{array}$ & $\begin{array}{c}\text { Phenol, } \\
\text { (C6h-50h) } \\
\text { total } \\
(\mu \mathrm{g} / \mathrm{L})\end{array}$ & $\begin{array}{l}\text { Ace- } \\
\text { naph- } \\
\text { thene, } \\
\text { total } \\
(\mu \mathrm{g} / \mathrm{L})\end{array}$ & $\begin{array}{c}\text { Ace- } \\
\text { naph- } \\
\text { thylene, } \\
\text { total } \\
(\mu \mathrm{g} / \mathrm{L})\end{array}$ & $\begin{array}{l}\text { Anthre- } \\
\text { cene, } \\
\text { total } \\
(\mu g / L)\end{array}$ & $\begin{array}{c}\text { Benzo A } \\
\text { anthra- } \\
\text { cene 1,2- } \\
\text { benzan- } \\
\text { thracene, } \\
\text { total } \\
(\mu \mathrm{g} / \mathrm{L})\end{array}$ & $\begin{array}{c}\text { Benzo B } \\
\text { fluor- } \\
\text { anthene, } \\
\text { total } \\
(\mu g / L)\end{array}$ & $\begin{array}{c}\text { Benzo K } \\
\text { fluor- } \\
\text { anthene, } \\
\text { total } \\
(\mu \mathrm{g} / \mathrm{L})\end{array}$ & $\begin{array}{c}\text { Benzo A } \\
\text { Pyrene, } \\
\text { total } \\
(\mu \mathrm{g} / \mathrm{L})\end{array}$ \\
\hline \multicolumn{10}{|c|}{ Tanque Verde Creek at Tucson } \\
\hline $02-02-88$ & $<30.0$ & $<5.0$ & $<5.0$ & $<5.0$ & $<5.0$ & $<5.0$ & $<10.0$ & $<10.0$ & $<10.0$ \\
\hline $08-20-88$ & $<30.0$ & $<5.0$ & $<5.0$ & $<5.0$ & $<5.0$ & $<5.0$ & $<10.0$ & $<10.0$ & $<10.0$ \\
\hline $07-20-90$ & $<30.0$ & $<5.0$ & $<5.0$ & $<5.0$ & $<5.0$ & $<10.0$ & $<10.0$ & $<10.0$ & $<10.0$ \\
\hline $07-24-90$ & $<30.0$ & $<5.0$ & $<5.0$ & $<5.0$ & $<5.0$ & $<10.0$ & $<10.0$ & $<10.0$ & $<10.0$ \\
\hline $01-05-91$ & $<30.0$ & $<5.0$ & $<5.0$ & $<5.0$ & $<5.0$ & $<10.0$ & $<10.0$ & $<10.0$ & $<10.0$ \\
\hline $01-06-92$ & $<30.0$ & $<5.0$ & $<5.0$ & $<5.0$ & $<5.0$ & $<10.0$ & $<10.0$ & $<10.0$ & $<10.0$ \\
\hline $02-09-92$ & $<30.0$ & $<5.0$ & $<5.0$ & $<5.0$ & $<5.0$ & $<10.0$ & $<10.0$ & $<10.0$ & $<10.0$ \\
\hline \multicolumn{10}{|c|}{ Pantano Wash at Broadray Boulevard } \\
\hline $11-01-87$ & $<30.0$ & $<5.0$ & $<5.0$ & $<5.0$ & $<5.0$ & $<5.0$ & $<10.0$ & $<10.0$ & $<10.0$ \\
\hline $02-13-92$ & $<30.0$ & $<5.0$ & $<5.0$ & $<5.0$ & $<5.0$ & $<5.0$ & $<10.0$ & $<10.0$ & $<10.0$ \\
\hline \multicolumn{10}{|c|}{ Alamo Wash at Glenn Street } \\
\hline $07-26-87$ & $<30.0$ & $<5.0$ & $<5.0$ & $<5.0$ & $<5.0$ & $<5.0$ & $<10.0$ & $<10.0$ & $<10.0$ \\
\hline $08-11-87$ & $<30.0$ & $<5.0$ & $<5.0$ & $<5.0$ & $<5.0$ & $<5.0$ & $<10.0$ & $<10.0$ & $<10.0$ \\
\hline $08-20-88$ & $<30.0$ & $<5.0$ & $<5.0$ & $<5.0$ & $<5.0$ & $<5.0$ & $<10.0$ & $<10.0$ & $<10.0$ \\
\hline $07-20-90$ & $<30.0$ & $<5.0$ & $<5.0$ & $<5.0$ & $<5.0$ & $<10.0$ & $<10.0$ & $<10.0$ & $<10.0$ \\
\hline $07-24-90$ & $<30.0$ & $<5.0$ & $<5.0$ & $<5.0$ & $<5.0$ & $<10.0$ & $<10.0$ & $<10.0$ & $<10.0$ \\
\hline $09-14-90$ & $<30.0$ & $<5.0$ & $<5.0$ & $<5.0$ & $<5.0$ & $<10.0$ & $<10.0$ & $<10.0$ & $<10.0$ \\
\hline $10-27-91$ & $<30.0$ & $<5.0$ & $<5.0$ & $<5.0$ & $<5.0$ & $<5.0$ & $<10.0$ & $<10.0$ & $<10.0$ \\
\hline $11-15-91$ & $<30.0$ & $<5.0$ & $<5.0$ & $<5.0$ & $<5.0$ & $<10.0$ & $<10.0$ & $<10.0$ & $<10.0$ \\
\hline $12-11-91$ & $<30.0$ & $<5.0$ & $<5.0$ & $<5.0$ & $<5.0$ & $<10.0$ & $<10.0$ & $<10.0$ & $<10.0$ \\
\hline 01-06-92 & $<30.0$ & $<5.0$ & $<5.0$ & $<5.0$ & $<5.0$ & $<10.0$ & $<10.0$ & $<10.0$ & $<10.0$ \\
\hline \multicolumn{10}{|c|}{ Rilito Creek at Dodge Boulevard } \\
\hline $07-30-88$ & $<30.0$ & $<5.0$ & $<5.0$ & $<5.0$ & $<5.0$ & $<5.0$ & $<10.0$ & $<10.0$ & $<10.0$ \\
\hline $08-20-88$ & $<30.0$ & $<5.0$ & $<5.0$ & $<5.0$ & $<5.0$ & $<5.0$ & $<10.0$ & $<10.0$ & $<10.0$ \\
\hline $07-20-90$ & $<30.0$ & $<5.0$ & $<5.0$ & $<5.0$ & $<5.0$ & $<10.0$ & $<10.0$ & $<10.0$ & $<10.0$ \\
\hline $01-05-91$ & $<30.0$ & $<5.0$ & $<5.0$ & $<5.0$ & $<5.0$ & $<10.0$ & $<10.0$ & $<10.0$ & $<10.0$ \\
\hline $02-09-92$ & $<30.0$ & $<5.0$ & $<5.0$ & $<5.0$ & $<5.0$ & $<10.0$ & $<10.0$ & $<10.0$ & $<10.0$ \\
\hline $02-13-92$ & $<30.0$ & $<5.0$ & $<5.0$ & $<5.0$ & $<5.0$ & $<10.0$ & $<10.0$ & $<10.0$ & $<10.0$ \\
\hline
\end{tabular}


Table 8. Analytical results of surface-water samples, Rillito Creek basin Priority pollutants-Continued

\begin{tabular}{|c|c|c|c|c|c|c|c|c|c|}
\hline Date & $\begin{array}{c}\text { Benzogh I } \\
\text { perylene, 1, } \\
\text { 12-benzo- } \\
\text { perylene, } \\
\text { total } \\
(\mu \mathrm{g} / \mathrm{L})\end{array}$ & $\begin{array}{c}\text { N-butyl- } \\
\text { benzly- } \\
\text { phthalate, } \\
\text { total } \\
(\mu g / L)\end{array}$ & $\begin{array}{c}\text { Bis (2- } \\
\text { chloro- } \\
\text { ethoxy) } \\
\text { methane, } \\
\text { total } \\
(\mu \mathrm{g} / \mathrm{L})\end{array}$ & $\begin{array}{l}\text { Bis (2- } \\
\text { chioro- } \\
\text { ethyl) } \\
\text { ether, } \\
\text { total } \\
(\mu g / \mathrm{L})\end{array}$ & $\begin{array}{c}\text { Bis(2- } \\
\text { chloro- } \\
\text { isopropyl) } \\
\text { ether, total } \\
(\mu g / L)\end{array}$ & $\begin{array}{c}\text { 4-Bromo- } \\
\text { phenyi } \\
\text { phenyl } \\
\text { ether, } \\
\text { total } \\
\text { ( } \mu \mathrm{g} / \mathrm{L})\end{array}$ & $\begin{array}{c}\text { 2-Chioro- } \\
\text { naph- } \\
\text { thalene, } \\
\text { total } \\
(\mu g / L)\end{array}$ & $\begin{array}{l}\text { 4-Chloro- } \\
\text { phenyi } \\
\text { phenyl } \\
\text { ether, } \\
\text { total } \\
(\mu \mathrm{g} / \mathrm{L})\end{array}$ & $\begin{array}{c}\text { Chrysene, } \\
\text { total } \\
(\mu \mathrm{g} / \mathrm{L})\end{array}$ \\
\hline \multicolumn{10}{|c|}{ Tanque Verde Creek at Tucson } \\
\hline $02-02-88$ & $<10.0$ & $<5.0$ & $<5.0$ & $<5.0$ & $<5.0$ & $<5.0$ & $<5.0$ & $<5.0$ & $<10.0$ \\
\hline $08-20-88$ & $<10.0$ & $<5.0$ & $<5.0$ & $<5.0$ & $<5.0$ & $<5.0$ & $<5.0$ & $<5.0$ & $<10.0$ \\
\hline $07-20-90$ & $<10.0$ & $<5.0$ & $<5.0$ & $<5.0$ & $<5.0$ & $<5.0$ & $<5.0$ & $<5.0$ & $<10.0$ \\
\hline $07-24-90$ & $<10.0$ & $<5.0$ & $<5.0$ & $<5.0$ & $<5.0$ & $<5.0$ & $<5.0$ & $<5.0$ & $<10.0$ \\
\hline 01-05-91 & $<10.0$ & $<5.0$ & $<5.0$ & $<5.0$ & $<5.0$ & $<5.0$ & $<5.0$ & $<5.0$ & $<10.0$ \\
\hline $01-06-92$ & $<10.0$ & $<5.0$ & $<5.0$ & $<5.0$ & $<5.0$ & $<5.0$ & $<5.0$ & $<5.0$ & $<10.0$ \\
\hline 02-09-92 & $<10.0$ & $<5.0$ & $<5.0$ & $<5.0$ & $<5.0$ & $<5.0$ & $<5.0$ & $<5.0$ & $<10.0$ \\
\hline \multicolumn{10}{|c|}{ Pantano Wash at Broadway Boulevard } \\
\hline $11-01-87$ & $<10.0$ & $<5.0$ & $<5.0$ & $<5.0$ & $<5.0$ & $<5.0$ & $<5.0$ & $<5.0$ & $<10.0$ \\
\hline 02-13-92 & $<10.0$ & $<5.0$ & $<5.0$ & $<5.0$ & $<5.0$ & $<5.0$ & $<5.0$ & $<5.0$ & $<10.0$ \\
\hline \multicolumn{10}{|c|}{ Alamo Wash at Glenn Streef } \\
\hline $07-26-87$ & $<10.0$ & $<5.0$ & $<5.0$ & $<5.0$ & $<5.0$ & $<5.0$ & $<5.0$ & $<5.0$ & $<10.0$ \\
\hline $08-11-87$ & $<10.0$ & $<5.0$ & $<5.0$ & $<5.0$ & $<5.0$ & $<5.0$ & $<5.0$ & $<5.0$ & $<10.0$ \\
\hline 08-20-88 & $<10.0$ & $<5.0$ & $<5.0$ & $<5.0$ & $<5.0$ & $<5.0$ & $<5.0$ & $<5.0$ & $<10.0$ \\
\hline $07-20-90$ & $<10.0$ & $<5.0$ & $<5.0$ & $<5.0$ & $<5.0$ & $<5.0$ & $<5.0$ & $<5.0$ & $<10.0$ \\
\hline $07-24-90$ & $<10.0$ & $<5.0$ & $<5.0$ & $<5.0$ & $<5.0$ & $<5.0$ & $<5.0$ & $<5.0$ & $<10.0$ \\
\hline $09-14-90$ & $<10.0$ & $<5.0$ & $<5.0$ & $<5.0$ & $<5.0$ & $<5.0$ & $<5.0$ & $<5.0$ & $<10.0$ \\
\hline $10-27-91$ & $<10.0$ & $<5.0$ & $<5.0$ & $<5.0$ & $<5.0$ & $<5.0$ & $<5.0$ & $<5.0$ & $<10.0$ \\
\hline $11-15-91$ & $<10.0$ & $<5.0$ & $<5.0$ & $<5.0$ & $<5.0$ & $<5.0$ & $<5.0$ & $<5.0$ & $<10.0$ \\
\hline $12-11-91$ & $<10.0$ & $<5.0$ & $<5.0$ & $<5.0$ & $<5.0$ & $<5.0$ & $<5.0$ & $<5.0$ & $<10.0$ \\
\hline $01-06-92$ & $<10.0$ & $<5.0$ & $<5.0$ & $<5.0$ & $<5.0$ & $<5.0$ & $<5.0$ & $<5.0$ & $<10.0$ \\
\hline \multicolumn{10}{|c|}{ Rilitito Creek at Dodge Boulevard } \\
\hline $07-30-88$ & $<10.0$ & $<5.0$ & $<5.0$ & $<5.0$ & $<5.0$ & $<5.0$ & $<5.0$ & $<5.0$ & $<10.0$ \\
\hline $08-20-88$ & $<10.0$ & $<5.0$ & $<5.0$ & $<5.0$ & $<5.0$ & $<5.0$ & $<5.0$ & $<5.0$ & $<10.0$ \\
\hline $07-20-90$ & $<10.0$ & $<5.0$ & $<5.0$ & $<5.0$ & $<5.0$ & $<5.0$ & $<5.0$ & $<5.0$ & $<10.0$ \\
\hline $01-05-91$ & $<10.0$ & $<5.0$ & $<5.0$ & $<5.0$ & $<5.0$ & $<5.0$ & $<5.0$ & $<5.0$ & $<10.0$ \\
\hline $02-09-92$ & $<10.0$ & $<5.0$ & $<5.0$ & $<5.0$ & $<5.0$ & $<5.0$ & $<5.0$ & $<5.0$ & $<10.0$ \\
\hline $02-13-92$ & $<10.0$ & $<5.0$ & $<5.0$ & $<5.0$ & $<5.0$ & $<5.0$ & $<5.0$ & $<5.0$ & $<10.0$ \\
\hline
\end{tabular}


Table 8. Analytical results of surface-water samples, Rillito Creek basin Priority pollutants-Continued

\begin{tabular}{|c|c|c|c|c|c|c|c|c|c|}
\hline Date & $\begin{array}{c}1,2,5,6 \\
\text { Dibenz- } \\
\text { anthra- } \\
\text { cene, } \\
\text { total } \\
(\mu \mathrm{g} / \mathrm{L})\end{array}$ & $\begin{array}{c}\text { Di-n- } \\
\text { butyl } \\
\text { phthal- } \\
\text { ate, } \\
\text { total } \\
(\mu \mathrm{g} / \mathrm{L})\end{array}$ & $\begin{array}{c}\text { 1,2-Di- } \\
\text { chloro- } \\
\text { benzene, } \\
\text { total } \\
(\mu \mathrm{g} / \mathrm{L})\end{array}$ & $\begin{array}{c}\text { 1,3-Di- } \\
\text { chioro- } \\
\text { benzene, } \\
\text { totai } \\
(\mu g / L)\end{array}$ & $\begin{array}{c}\text { 1,4-Di- } \\
\text { chioro- } \\
\text { benzene, } \\
\text { totai } \\
(\mu g / L)\end{array}$ & $\begin{array}{c}\text { Di- } \\
\text { ethyi- } \\
\text { phthai- } \\
\text { ate, } \\
\text { totai } \\
(\mu g / L)\end{array}$ & $\begin{array}{c}\text { Di- } \\
\text { methyi- } \\
\text { phthai- } \\
\text { ate, } \\
\text { totai } \\
(\mu g / L)\end{array}$ & $\begin{array}{c}\text { 2,4-Di- } \\
\text { nitro- } \\
\text { toluene, } \\
\text { totai } \\
(\mu g / L)\end{array}$ & $\begin{array}{c}2,6-\mathrm{Di}- \\
\text { nitro- } \\
\text { toluene, } \\
\text { total } \\
(\mu \mathrm{g} / \mathrm{L})\end{array}$ \\
\hline \multicolumn{10}{|c|}{ Tanque Verde Creek at Tueson } \\
\hline $02-02-88$ & $<10.0$ & $<.0$ & $<5.0$ & $<5.0$ & $<5.0$ & $<5.0$ & $<5.0$ & $<5.0$ & $<5.0$ \\
\hline $08-20-88$ & $<10.0$ & $<5.0$ & $<5.0$ & $<5.0$ & $<5.0$ & $<5.0$ & $<5.0$ & $<5.0$ & $<5.0$ \\
\hline $07-20-90$ & $<10.0$ & $<5.0$ & $<5.0$ & $<5.0$ & $<5.0$ & $<5.0$ & $<5.0$ & $<5.0$ & $<5.0$ \\
\hline $07-24-90$ & $<10.0$ & $<5.0$ & $<5.0$ & $<5.0$ & $<5.0$ & $<5.0$ & $<5.0$ & $<5.0$ & $<5.0$ \\
\hline $01-05-91$ & $<10.0$ & $<5.0$ & $<5.0$ & $<5.0$ & $<5.0$ & $<5.0$ & $<5.0$ & $<5.0$ & $<5.0$ \\
\hline $01-06-92$ & $<10.0$ & $<5.0$ & $<5.0$ & $<5.0$ & $<5.0$ & $<5.0$ & $<5.0$ & $<5.0$ & $<5.0$ \\
\hline $02-09-92$ & $<10.0$ & $<5.0$ & $<5.0$ & $<5.0$ & $<5.0$ & $<5.0$ & $<5.0$ & $<5.0$ & $<5.0$ \\
\hline \multicolumn{10}{|c|}{ Pantano Wash at Broadway Boulevard } \\
\hline $11-01-87$ & $<10.0$ & $<5.0$ & $<5.0$ & $<5.0$ & $<5.0$ & $<5.0$ & $<5.0$ & $<5.0$ & $<5.0$ \\
\hline $02-13-92$ & $<10.0$ & $<5.0$ & $<5.0$ & $<5.0$ & $<5.0$ & $<5.0$ & $<5.0$ & $<5.0$ & $<5.0$ \\
\hline \multicolumn{10}{|c|}{ Alamo Wash at Glenn Street } \\
\hline $07-26-87$ & $<10.0$ & $<5.0$ & $<5.0$ & $<5.0$ & $<5.0$ & $<5.0$ & $<5.0$ & $<5.0$ & $<5.0$ \\
\hline $08-11-87$ & $<10.0$ & $<5.0$ & $<5.0$ & $<5.0$ & $<5.0$ & $<5.0$ & $<5.0$ & $<5.0$ & $<5.0$ \\
\hline $08-20-88$ & $<10.0$ & $<5.0$ & $<5.0$ & $<5.0$ & $<5.0$ & $<5.0$ & $<5.0$ & $<5.0$ & $<5.0$ \\
\hline $07-20-90$ & $<10.0$ & $<5.0$ & $<5.0$ & $<5.0$ & $<5.0$ & $<5.0$ & $<5.0$ & $<5.0$ & $<5.0$ \\
\hline $07-24-90$ & $<10.0$ & $<5.0$ & $<5.0$ & $<5.0$ & $<5.0$ & $<5.0$ & $<5.0$ & $<5.0$ & $<5.0$ \\
\hline $09-14-90$ & $<10.0$ & $<5.0$ & $<5.0$ & $<5.0$ & $<5.0$ & $<5.0$ & $<5.0$ & $<5.0$ & $<5.0$ \\
\hline $10-27-91$ & $<10.0$ & $<5.0$ & $<5.0$ & $<5.0$ & $<5.0$ & $<5.0$ & $<5.0$ & $<5.0$ & $<5.0$ \\
\hline $11-15-91$ & $<10.0$ & $<5.0$ & $<5.0$ & $<5.0$ & $<5.0$ & $<5.0$ & $<5.0$ & $<5.0$ & $<5.0$ \\
\hline $12-11-91$ & $<10.0$ & $<5.0$ & $<5.0$ & $<5.0$ & $<5.0$ & $<5.0$ & $<5.0$ & $<5.0$ & $<5.0$ \\
\hline $01-06-92$ & $<10.0$ & $<5.0$ & $<5.0$ & $<5.0$ & $<5.0$ & $<5.0$ & $<5.0$ & $<5.0$ & $<5.0$ \\
\hline \multicolumn{10}{|c|}{ Rillito Creek at Dodge Boulevard } \\
\hline $07-30-88$ & $<10.0$ & $<5.0$ & $<5.0$ & $<5.0$ & $<5.0$ & $<5.0$ & $<5.0$ & $<5.0$ & $<5.0$ \\
\hline $08-20-88$ & $<10.0$ & $<5.0$ & $<5.0$ & $<5.0$ & $<5.0$ & $<5.0$ & $<5.0$ & $<5.0$ & $<5.0$ \\
\hline $07-20-90$ & $<10.0$ & $<5.0$ & $<5.0$ & $<5.0$ & $<5.0$ & $<5.0$ & $<5.0$ & $<5.0$ & $<5.0$ \\
\hline $01-05-91$ & $<10.0$ & $<5.0$ & $<5.0$ & $<5.0$ & $<5.0$ & $<5.0$ & $<5.0$ & $<5.0$ & $<5.0$ \\
\hline $02-09-92$ & $<10.0$ & $<5.0$ & $<5.0$ & $<5.0$ & $<5.0$ & $<5.0$ & $<5.0$ & $<5.0$ & $<5.0$ \\
\hline $02-13-92$ & $<10.0$ & $<5.0$ & $<5.0$ & $<5.0$ & $<5.0$ & $<5.0$ & $<5.0$ & $<5.0$ & $<5.0$ \\
\hline
\end{tabular}


Tabie 8. Analytical results of surface-water samples, Rillito Creek basin Priority pollutants-Continued

\begin{tabular}{|c|c|c|c|c|c|c|c|c|c|}
\hline Date & $\begin{array}{c}\text { Di-n- } \\
\text { octyl } \\
\text { phthal- } \\
\text { ate, } \\
\text { total } \\
(\mu \mathrm{g} / \mathrm{L})\end{array}$ & $\begin{array}{c}\text { Bis (2- } \\
\text { ethyl- } \\
\text { hexyl) } \\
\text { phthal- } \\
\text { ate, } \\
\text { total } \\
(\mu g / L)\end{array}$ & $\begin{array}{c}\text { Fluorene, } \\
\text { total } \\
(\mu \mathrm{g} / \mathrm{L})\end{array}$ & $\begin{array}{c}\text { Fluor- } \\
\text { anthene, } \\
\text { total } \\
(\mu \mathrm{g} / L)\end{array}$ & $\begin{array}{c}\text { Hexa- } \\
\text { chlorom } \\
\text { benzene, } \\
\text { total } \\
(\mu \mathrm{g} / \mathrm{L})\end{array}$ & $\begin{array}{c}\text { Hexa- } \\
\text { chloro- } \\
\text { buta- } \\
\text { dlene, } \\
\text { total } \\
(\mu g / L)\end{array}$ & $\begin{array}{c}\text { Hexa- } \\
\text { chloro- } \\
\text { cyclo- } \\
\text { penta- } \\
\text { dlene, } \\
\text { total } \\
(\mu \mathrm{g} / \mathrm{L})\end{array}$ & $\begin{array}{c}\text { Hexa- } \\
\text { chloro- } \\
\text { ethane, } \\
\text { total } \\
(\mu \mathrm{g} / \mathrm{L})\end{array}$ & $\begin{array}{c}\text { Indeno } \\
(1,2,3-c d) \\
\text { pyrene, } \\
\text { total } \\
(\mu g / L)\end{array}$ \\
\hline \multicolumn{10}{|c|}{ Tanque Verde Creek at Tucson } \\
\hline $02-02-88$ & $<10.0$ & $<5.0$ & $<5.0$ & $<5.0$ & $<5.0$ & $<5.0$ & $<5.0$ & $<5.0$ & $<10.0$ \\
\hline $08-20-88$ & $<10.0$ & $<5.0$ & $<5.0$ & $<5.0$ & $<5.0$ & $<5.0$ & $<5.0$ & $<5.0$ & $<10.0$ \\
\hline $07-20-90$ & $<10.0$ & $\cdots$ & $<5.0$ & $<5.0$ & $<5.0$ & $<5.0$ & $<5.0$ & $<5.0$ & $<10.0$ \\
\hline $04-24-90$ & $<10.0$ & $<5.0$ & $<5.0$ & $<5.0$ & $<5.0$ & $<5.0$ & $<5.0$ & $<5.0$ & $<10.0$ \\
\hline $01-05-91$ & $<10.0$ & $<5.0$ & $<5.0$ & $<5.0$ & $<5.0$ & $<5.0$ & $<5.0$ & $<5.0$ & $<10.0$ \\
\hline $01-06-92$ & $<10.0$ & $<5.0$ & $<5.0$ & $<5.0$ & $<5.0$ & $<5.0$ & $<5.0$ & $<5.0$ & $<10.0$ \\
\hline $02-09-92$ & $<10.0$ & $<5.0$ & $<5.0$ & $<5.0$ & $<5.0$ & $<5.0$ & $<5.0$ & $<5.0$ & $<10.0$ \\
\hline \multicolumn{10}{|c|}{ Pantano Wash at Broadway Boulevard } \\
\hline 11-01-87 & $<10.0$ & $<5.0$ & $<5.0$ & $<5.0$ & $<5.0$ & $<5.0$ & $<5.0$ & $<5.0$ & $<10.0$ \\
\hline $02-13-92$ & $<10.0$ & $<5.0$ & $<5.0$ & $<5.0$ & $<5.0$ & $<5.0$ & $<5.0$ & $<5.0$ & $<10.0$ \\
\hline \multicolumn{10}{|c|}{ Alamo Wash at Glenn Street } \\
\hline $07-26-87$ & $<10.0$ & $<5.0$ & $<5.0$ & $<5.0$ & $<5.0$ & $<5.0$ & $<5.0$ & $<5.0$ & $<10.0$ \\
\hline $08-11-87$ & $<10.0$ & $<5.0$ & $<5.0$ & $<5.0$ & $<5.0$ & $<5.0$ & $<5.0$ & $<5.0$ & $<10.0$ \\
\hline $08-20-88$ & $<10.0$ & $<7.0$ & $<5.0$ & 5.0 & $<5.0$ & $<5.0$ & $<5.0$ & $<5.0$ & $<10.0$ \\
\hline $07-20-90$ & $<10.0$ & ---- & $<5.0$ & 6.0 & $<5.0$ & $<5.0$ & $<5.0$ & $<5.0$ & $<10.0$ \\
\hline $07-24-90$ & $<10.0$ & 10.0 & $<5.0$ & 6.0 & $<5.0$ & $<5.0$ & $<5.0$ & $<5.0$ & $<10.0$ \\
\hline $09-14-90$ & $<10.0$ & 6.0 & $<5.0$ & 5.0 & $<5.0$ & $<5.0$ & $<5.0$ & $<5.0$ & $<10.0$ \\
\hline $10-27-91$ & $<10.0$ & $<5.0$ & $<5.0$ & $<5.0$ & $<5.0$ & $<5.0$ & $<5.0$ & $<5.0$ & $<10.0$ \\
\hline $11-15-91$ & $<10.0$ & $<5.0$ & $<5.0$ & $<5.0$ & $<5.0$ & $<5.0$ & $<5.0$ & $<5.0$ & $<10.0$ \\
\hline $12-11-91$ & $<10.0$ & $<5.0$ & $<5.0$ & $<5.0$ & $<5.0$ & $<5.0$ & $<5.0$ & $<5.0$ & $<10.0$ \\
\hline $01-06-92$ & $<10.0$ & 6.0 & $<5.0$ & 5.1 & $<5.0$ & $<5.0$ & $<5.0$ & $<5.0$ & $<10.0$ \\
\hline \multicolumn{10}{|c|}{ Rillito Creek at Dodge Boulerard } \\
\hline $07-30-88$ & $<10.0$ & $<13.0$ & $<5.0$ & $<5.0$ & $<5.0$ & $<5.0$ & $<5.0$ & $<5.0$ & $<10.0$ \\
\hline $08-20-88$ & $<10.0$ & $<5.0$ & $<5.0$ & $<5.0$ & $<5.0$ & $<5.0$ & $<5.0$ & $<5.0$ & $<10.0$ \\
\hline $07-20-90$ & $<10.0$ & 7.0 & $<5.0$ & $<5.0$ & $<5.0$ & $<5.0$ & $<5.0$ & $<5.0$ & $<10.0$ \\
\hline $01-05-91$ & $<10.0$ & 11.0 & $<5.0$ & $<5.0$ & $<5.0$ & $<5.0$ & $<5.0$ & $<5.0$ & $<10.0$ \\
\hline $02-09-92$ & $<10.0$ & $<5.0$ & $<5.0$ & $<5.0$ & $<5.0$ & $<5.0$ & $<5.0$ & $<5.0$ & $<10.0$ \\
\hline $02-13-92$ & $<10.0$ & $<5.0$ & $<5.0$ & $<5.0$ & $<5.0$ & $<5.0$ & $<5.0$ & $<5.0$ & $<10.0$ \\
\hline
\end{tabular}


Table 8. Analytical results of surface-water samples, Rillito Creek basin Priority pollutants-Continued

\begin{tabular}{|c|c|c|c|c|c|c|c|c|c|}
\hline Date & $\begin{array}{c}\text { Iso- } \\
\text { phorone, } \\
\text { total } \\
(\mu \mathrm{g} / \mathrm{L})\end{array}$ & $\begin{array}{c}\text { Naph- } \\
\text { thalene, } \\
\text { total } \\
(\mu \mathrm{g} / \mathrm{L})\end{array}$ & $\begin{array}{c}\text { Nitro- } \\
\text { benzene, } \\
\text { total } \\
(\mu g / L)\end{array}$ & $\begin{array}{l}\text { N-nitro- } \\
\text { sodi- } \\
\text { methyl- } \\
\text { amine, } \\
\text { total } \\
(\mu \mathrm{g} / \mathrm{L})\end{array}$ & $\begin{array}{l}\text { N-nitro- } \\
\text { sodi- } \\
\text { phenyl- } \\
\text { amine, } \\
\text { total } \\
(\mu \mathrm{g} / \mathrm{L})\end{array}$ & $\begin{array}{c}\text { N-nitro- } \\
\text { sodi-n- } \\
\text { propyl- } \\
\text { amine, } \\
\text { total } \\
(\mu \mathrm{g} / L)\end{array}$ & $\begin{array}{c}\text { Phenan- } \\
\text { threne, } \\
\text { total } \\
(\mu \mathrm{g} / \mathrm{L})\end{array}$ & $\begin{array}{c}\text { Pyrene, } \\
\text { total } \\
(\mu g / L)\end{array}$ & $\begin{array}{c}\text { 1,2,4- } \\
\text { Trichloro- } \\
\text { benzene, } \\
\text { total } \\
(\mu \mathrm{g} / \mathrm{L})\end{array}$ \\
\hline \multicolumn{10}{|c|}{ Tanque Verde Creek at Tucson } \\
\hline $02-02-88$ & $<5.0$ & $<5.0$ & $<5.0$ & $<5.0$ & $<5.0$ & $<5.0$ & $<5.0$ & $<5.0$ & $<5.0$ \\
\hline $02-20-88$ & $<5.0$ & $<5.0$ & $<5.0$ & $<5.0$ & $<5.0$ & $<5.0$ & $<5.0$ & $<5.0$ & $<5.0$ \\
\hline $07-20-90$ & $<5.0$ & $<5.0$ & $<5.0$ & $<5.0$ & $<5.0$ & $<5.0$ & $<5.0$ & $<5.0$ & $<5.0$ \\
\hline $07-24-90$ & $<5.0$ & $<5.0$ & $<5.0$ & $<5.0$ & $<5.0$ & $<5.0$ & $<5.0$ & $<5.0$ & $<5.0$ \\
\hline $01-05-91$ & $<5.0$ & $<5.0$ & $<5.0$ & $<5.0$ & $<5.0$ & $<5.0$ & $<5.0$ & $<5.0$ & $<5.0$ \\
\hline $01-06-92$ & $<5.0$ & $<5.0$ & $<5.0$ & $<5.0$ & $<5.0$ & $<5.0$ & $<5.0$ & $<5.0$ & $<5.0$ \\
\hline $02-09-92$ & $<5.0$ & $<5.0$ & $<5.0$ & $<5.0$ & $<5.0$ & $<5.0$ & $<5.0$ & $<5.0$ & $<5.0$ \\
\hline \multicolumn{10}{|c|}{ Pantano Wash at Broadway Boulevard } \\
\hline $11-01-87$ & $<5.0$ & $<5.0$ & $<5.0$ & $<5.0$ & $<5.0$ & $<5.0$ & $<5.0$ & $<5.0$ & $<5.0$ \\
\hline $02-13-92$ & $<5.0$ & $<5.0$ & $<5.0$ & $<5.0$ & $<5.0$ & $<5.0$ & $<5.0$ & $<5.0$ & $<5.0$ \\
\hline \multicolumn{10}{|c|}{ Alamo Wash at Glenn Street } \\
\hline $07-26-87$ & $<5.0$ & $<5.0$ & $<5.0$ & $<5.0$ & $<5.0$ & $<5.0$ & $<5.0$ & $<5.0$ & $<5.0$ \\
\hline $08-11-87$ & $<5.0$ & $<5.0$ & $<5.0$ & $<5.0$ & $<5.0$ & $<5.0$ & $<5.0$ & $<5.0$ & $<5.0$ \\
\hline $09-20-88$ & $<5.0$ & $<5.0$ & $<5.0$ & $<5.0$ & $<5.0$ & $<5.0$ & $<5.0$ & 6.0 & $<5.0$ \\
\hline $07-24-90$ & $<5.0$ & $<5.0$ & $<5.0$ & $<5.0$ & $<5.0$ & $<5.0$ & $<5.0$ & 5.0 & $<5.0$ \\
\hline $07-14-90$ & $<5.0$ & $<5.0$ & $<5.0$ & $<5.0$ & $<5.0$ & $<5.0$ & $<5.0$ & $<5.0$ & $<5.0$ \\
\hline $09-27-90$ & $<5.0$ & $<5.0$ & $<5.0$ & $<5.0$ & $<5.0$ & $<5.0$ & $<5.0$ & $<5.0$ & $<5.0$ \\
\hline $10-27-91$ & $<5.0$ & $<5.0$ & $<5.0$ & $<5.0$ & $<5.0$ & $<5.0$ & $<5.0$ & 6.0 & $<5.0$ \\
\hline $11-15-91$ & $<5.0$ & $<5.0$ & $<5.0$ & $<5.0$ & $<5.0$ & $<5.0$ & $<5.0$ & 5.0 & $<5.0$ \\
\hline $12-11-91$ & $<5.0$ & $<5.0$ & $<5.0$ & $<5.0$ & $<5.0$ & $<5.0$ & $<5.0$ & $<5.0$ & $<5.0$ \\
\hline $01-06-92$ & $<5.0$ & $<5.0$ & $<5.0$ & $<5.0$ & $<5.0$ & $<5.0$ & $<5.0$ & $<5.0$ & $<5.0$ \\
\hline \multicolumn{10}{|c|}{ Rillito Creek at Dodge Boulevard } \\
\hline $07-30-88$ & $<5.0$ & $<5.0$ & $<5.0$ & $<5.0$ & $<5.0$ & $<5.0$ & $<5.0$ & $<5.0$ & $<5.0$ \\
\hline $08-20-88$ & $<5.0$ & $<5.0$ & $<5.0$ & $<5.0$ & $<5.0$ & $<5.0$ & $<5.0$ & $<5.0$ & $<5.0$ \\
\hline $07-20-90$ & $<5.0$ & $<5.0$ & $<5.0$ & $<5.0$ & $<5.0$ & $<5.0$ & $<5.0$ & $<5.0$ & $<5.0$ \\
\hline $01-05-91$ & $<5.0$ & $<5.0$ & $<5.0$ & $<5.0$ & $<5.0$ & $<5.0$ & $<5.0$ & $<5.0$ & $<5.0$ \\
\hline $01-09-92$ & $<5.0$ & $<5.0$ & $<5.0$ & $<5.0$ & $<5.0$ & $<5.0$ & $<5.0$ & $<5.0$ & $<5.0$ \\
\hline $02-13-92$ & $<5.0$ & $<5.0$ & $<5.0$ & $<5.0$ & $<5.0$ & $<5.0$ & $<5.0$ & $<5.0$ & $<5.0$ \\
\hline
\end{tabular}


Table 8. Analytical results of surface-water samples, Rillito Creek basin-Continued Volatile organic compounds

\begin{tabular}{|c|c|c|c|c|c|c|c|c|c|}
\hline Date & $\begin{array}{c}\text { Dis- } \\
\text { charge, } \\
\text { Instan- } \\
\text { taneous, } \\
\left(\mathrm{ft}^{3} / \mathrm{s}\right)\end{array}$ & $\begin{array}{c}\text { Benzene, } \\
\text { total } \\
(\mu g / L)\end{array}$ & $\begin{array}{l}\text { Bromo- } \\
\text { form, } \\
\text { total } \\
(\mu g / L)\end{array}$ & $\begin{array}{l}\text { Carbon- } \\
\text { tetrachlo- } \\
\text { ride, } \\
\text { total } \\
(\mu g / L)\end{array}$ & $\begin{array}{c}\text { Chloro- } \\
\text { benzene, } \\
\text { total } \\
(\mu g / L)\end{array}$ & $\begin{array}{c}\text { Chloro- } \\
\text { dlbromo- } \\
\text { methane, } \\
\text { total } \\
(\mu g / L)\end{array}$ & $\begin{array}{c}\text { Chloro- } \\
\text { ethane, } \\
\text { total } \\
(\mu \mathrm{g} / L)\end{array}$ & $\begin{array}{l}\text { 2-Chloro- } \\
\text { ethylvinyl- } \\
\text { ether, } \\
\text { total } \\
(\mu \mathrm{g} / \mathrm{L})\end{array}$ & $\begin{array}{c}\text { Chloro- } \\
\text { form, } \\
\text { total } \\
(\mu g / L)\end{array}$ \\
\hline \multicolumn{10}{|c|}{ Tanque Verde Creek at Tucson } \\
\hline $02-02-88$ & 44 & $<3.0$ & $<3.0$ & $<3.0$ & $<3.0$ & $<3.0$ & $<3.0$ & $<3.0$ & $<3.0$ \\
\hline $01-06-92$ & 105 & $<3.0$ & $<3.0$ & $<3.0$ & $<3.0$ & $<3.0$ & $<3.0$ & $<3.0$ & $<3.0$ \\
\hline \multicolumn{10}{|c|}{ Pantano Wash at Broadway Boulevard } \\
\hline $11-01-87$ & 50 & $<3.0$ & $<3.0$ & $<3.0$ & $<3.0$ & $<3.0$ & $<3.0$ & $<3.0$ & $<3.0$ \\
\hline $07-24-90$ & 4,470 & $<3.0$ & $<3.0$ & $<3.0$ & $<3.0$ & $<3.0$ & $<3.0$ & $<3.0$ & $<3.0$ \\
\hline $02-13-92$ & 97 & $<3.0$ & $<3.0$ & $<3.0$ & $<3.0$ & $<3.0$ & $<3.0$ & $<3.0$ & $<3.0$ \\
\hline \multicolumn{10}{|c|}{ Alame Wash at Glenn Street } \\
\hline 09-04-87 & 58 & $<3.0$ & $<3.0$ & $<3.0$ & $<3.0$ & $<3.0$ & $<3.0$ & $<3.0$ & $<3.0$ \\
\hline $11-15-91$ & 33 & $<3.0$ & $<3.0$ & $<3.0$ & $<3.0$ & $<3.0$ & $<3.0$ & $<3.0$ & $<3.0$ \\
\hline 01-06-92 & 325 & $<3.0$ & $<3.0$ & $<3.0$ & $<3.0$ & $<3.0$ & $<3.0$ & $<3.0$ & $<3.0$ \\
\hline \multicolumn{10}{|c|}{ Rilito Creek at Dodge Boulevard } \\
\hline $07-30-88$ & 5,300 & $<3.0$ & $<3.0$ & $<3.0$ & $<3.0$ & $<3.0$ & $<3.0$ & $<3.0$ & $<3.0$ \\
\hline $02-09-92$ & 95 & $<3.0$ & $<3.0$ & $<3.0$ & $<3.0$ & $<3.0$ & $<3.0$ & $<3.0$ & $<3.0$ \\
\hline
\end{tabular}

\begin{tabular}{|c|c|c|c|c|c|c|c|c|c|}
\hline Date & $\begin{array}{c}\text { DI- } \\
\text { chloro- } \\
\text { bromo- } \\
\text { methane, } \\
\text { total } \\
(\mu \mathrm{g} / \mathrm{L})\end{array}$ & $\begin{array}{c}\text { Di- } \\
\text { chlorodl- } \\
\text { fluoro- } \\
\text { methane, } \\
\text { total } \\
(\mu g / L)\end{array}$ & $\begin{array}{c}\text { 1,1-Di- } \\
\text { chloro- } \\
\text { ethane, } \\
\text { total } \\
(\mu g / L)\end{array}$ & $\begin{array}{c}\text { 1,2-DI- } \\
\text { chloro- } \\
\text { ethane, } \\
\text { total } \\
(\mu \mathrm{g} / \mathrm{L})\end{array}$ & $\begin{array}{c}\text { 1,1-DI- } \\
\text { chloro- } \\
\text { ethylene, } \\
\text { total } \\
(\mu \mathrm{g} / \mathrm{L})\end{array}$ & $\begin{array}{c}1,2- \\
\text { Transdi- } \\
\text { chloro- } \\
\text { ethane, } \\
\text { total } \\
(\mu \mathrm{g} / \mathrm{L})\end{array}$ & $\begin{array}{c}\text { 1,2-DI- } \\
\text { chloro- } \\
\text { propane, } \\
\text { total } \\
(\mu g / L)\end{array}$ & $\begin{array}{c}\text { 1,3-DI- } \\
\text { chloro- } \\
\text { propene, } \\
\text { total } \\
(\mu g / L)\end{array}$ & $\begin{array}{c}\text { Ethyl- } \\
\text { benzene, } \\
\text { total } \\
(\mu \mathrm{g} / \mathrm{L})\end{array}$ \\
\hline \multicolumn{10}{|c|}{ Tanque Verde Creek at Tucson } \\
\hline $02-02-88$ & $<3.0$ & $<3.0$ & $<3.0$ & $<3.0$ & $<3.0$ & $<3.0$ & $<3.0$ & $<3.0$ & $<3.0$ \\
\hline $01-06-92$ & $<3.0$ & $<3.0$ & $<3.0$ & $<3.0$ & $<3.0$ & $<3.0$ & $<3.0$ & -- & $<3.0$ \\
\hline \multicolumn{10}{|c|}{ Pantano Wash at Broadway Boulevard } \\
\hline $11-01-87$ & $<3.0$ & $<3.0$ & $<3.0$ & $<3.0$ & $<3.0$ & $<3.0$ & $<3.0$ & $<3.0$ & $<3.0$ \\
\hline $07-24-90$ & $<3.0$ & $<3.0$ & $<3.0$ & $<3.0$ & $<3.0$ & $<3.0$ & $<3.0$ & $<3.0$ & $<3.0$ \\
\hline 02-13-92 & $<3.0$ & $<3.0$ & $<3.0$ & $<3.0$ & $<3.0$ & $<3.0$ & $<3.0$ & - & $<3.0$ \\
\hline \multicolumn{10}{|c|}{ Alamo Wash at Glenn Street } \\
\hline 09-04-87 & $<3.0$ & $<3.0$ & $<3.0$ & $<3.0$ & $<3.0$ & $<3.0$ & $<3.0$ & $<3.0$ & $<3.0$ \\
\hline $11-15-91$ & $<3.0$ & $<3.0$ & $<3.0$ & $<3.0$ & $<3.0$ & $<3.0$ & $<3.0$ & -- & $<3.0$ \\
\hline $01-06-92$ & $<3.0$ & $<3.0$ & $<3.0$ & $<3.0$ & $<3.0$ & $<3.0$ & $<3.0$ & --- & $<3.0$ \\
\hline \multicolumn{10}{|c|}{ Rilito Creek at Dodge Boulevard } \\
\hline $07-30-88$ & $<3.0$ & $<3.0$ & $<3.0$ & $<3.0$ & $<3.0$ & $<3.0$ & $<3.0$ & $<3.0$ & $<3.0$ \\
\hline $02-09-92$ & $<3.0$ & $<3.0$ & $<3.0$ & $<3.0$ & $<3.0$ & $<3.0$ & $<3.0$ & --- & $<3.0$ \\
\hline
\end{tabular}


Table 8. Analytical results of surface-water samples, Rillito Creek basin-Continued Volatile organic compounds-Continued

\begin{tabular}{|c|c|c|c|c|c|c|c|c|c|}
\hline Date & $\begin{array}{c}\text { Methyl- } \\
\text { bromide, } \\
\text { total } \\
(\mu g / L)\end{array}$ & $\begin{array}{l}\text { Methyl- } \\
\text { ene, } \\
\text { chloride, } \\
\text { total } \\
(\mu g / L)\end{array}$ & $\begin{array}{c}1,1,2,2- \\
\text { Tetra- } \\
\text { chloro- } \\
\text { ethane, } \\
\text { total } \\
(\mu g / L)\end{array}$ & $\begin{array}{l}\text { Tetra- } \\
\text { chloro- } \\
\text { ethylene, } \\
\text { total } \\
(\mu \mathrm{g} / \mathrm{L})\end{array}$ & $\begin{array}{c}\text { Toluene, } \\
\text { total } \\
(\mu \mathrm{g} / \mathrm{L})\end{array}$ & $\begin{array}{c}1,1,1-T r l- \\
\text { chloro- } \\
\text { ethane, } \\
\text { total } \\
(\mu \mathrm{g} / \mathrm{L})\end{array}$ & $\begin{array}{c}\text { 1,1,2-Trl- } \\
\text { chloro- } \\
\text { ethane, } \\
\text { total } \\
(\mu g / L)\end{array}$ & $\begin{array}{c}\text { Tri- } \\
\text { chloro- } \\
\text { ethylene, } \\
\text { total } \\
(\mu g / L)\end{array}$ & $\begin{array}{c}\text { Tri- } \\
\text { chloro- } \\
\text { fluoro- } \\
\text { methane, } \\
\text { total } \\
(\mu \mathrm{g} / L)\end{array}$ \\
\hline \multicolumn{10}{|c|}{ Taqque Verde Creek at Tucson } \\
\hline $02-02-88$ & $<3.0$ & $<3.0$ & $<3.0$ & $<3.0$ & $<3.0$ & $<3.0$ & $<3.0$ & $<3.0$ & $<3.0$ \\
\hline $01-06-92$ & $<3.0$ & $<3.0$ & $<3.0$ & $<3.0$ & $<3.0$ & $<3.0$ & $<3.0$ & $<3.0$ & $<3.0$ \\
\hline \multicolumn{10}{|c|}{ Pantano Wash at Broadway Boulevard } \\
\hline $11-01-87$ & $<3.0$ & $<3.0$ & $<3.0$ & $<3.0$ & $<3.0$ & $<3.0$ & $<3.0$ & $<3.0$ & $<3.0$ \\
\hline $07-24-90$ & $<3.0$ & $<3.0$ & $<3.0$ & $<3.0$ & $<3.0$ & $<3.0$ & $<3.0$ & $<3.0$ & $<3.0$ \\
\hline $02-13-92$ & $<3.0$ & $<3.0$ & $<3.0$ & $<3.0$ & $<3.0$ & $<3.0$ & $<3.0$ & $<3.0$ & $<3.0$ \\
\hline \multicolumn{10}{|c|}{ Alamo Wash at Glenn Street } \\
\hline $09-04-87$ & $<3.0$ & $<3.0$ & $<3.0$ & $<3.0$ & $<3.0$ & $<3.0$ & $<3.0$ & $<3.0$ & $<3.0$ \\
\hline $11-15-91$ & $<3.0$ & $<3.0$ & $<3.0$ & $<3.0$ & $<3.0$ & $<3.0$ & $<3.0$ & $<3.0$ & $<3.0$ \\
\hline $01-06-92$ & $<3.0$ & $<3.0$ & $<3.0$ & $<3.0$ & $<3.0$ & $<3.0$ & $<3.0$ & $<3.0$ & $<3.0$ \\
\hline \multicolumn{10}{|c|}{ Rillito Creek at Dodge Boulevard } \\
\hline $07-30-88$ & $<3.0$ & $<3.0$ & $<3.0$ & $<3.0$ & $<3.0$ & $<3.0$ & $<3.0$ & $<3.0$ & $<3.0$ \\
\hline $02-09-92$ & $<3.0$ & $<3.0$ & $<3.0$ & $<3.0$ & $<3.0$ & $<3.0$ & $<3.0$ & $<3.0$ & $<3.0$ \\
\hline
\end{tabular}

\begin{tabular}{|c|c|c|c|c|c|c|c|c|c|c|}
\hline Date & $\begin{array}{l}\text { Vinyl } \\
\text { chlo- } \\
\text { ride, } \\
\text { total } \\
(\mu \mathrm{g} / \mathrm{L})\end{array}$ & $\begin{array}{c}\text { Methyl- } \\
\text { chloride, } \\
\text { total } \\
(\mu \mathrm{g} / \mathrm{L})\end{array}$ & $\begin{array}{c}\text { 1,2-Di- } \\
\text { bromo- } \\
\text { ethane, } \\
\text { total } \\
(\mu \mathrm{g} / \mathrm{L})\end{array}$ & $\begin{array}{c}\text { 1,2-Di- } \\
\text { chloro- } \\
\text { benzene, } \\
\text { total } \\
(\mu \mathrm{g} / L)\end{array}$ & $\begin{array}{c}\text { 1,3-Di- } \\
\text { chloro- } \\
\text { benzene, } \\
\text { total } \\
(\mu \mathrm{g} / \mathrm{L})\end{array}$ & $\begin{array}{c}\text { 1,4-Di- } \\
\text { chloro- } \\
\text { benzene, } \\
\text { total } \\
(\mu g / L)\end{array}$ & $\begin{array}{c}\text { Cls } \\
\text { 1,3-Di- } \\
\text { chloro- } \\
\text { propene, } \\
\text { total } \\
(\mu \mathrm{g} / \mathrm{L})\end{array}$ & $\begin{array}{c}\text { Trans- } \\
\text { 1,3-Di- } \\
\text { chloro- } \\
\text { propene, } \\
\text { total } \\
(\mu g / L)\end{array}$ & $\begin{array}{c}\text { Styrene, } \\
\text { total } \\
(\mu g / L)\end{array}$ & $\begin{array}{c}\text { Xylene, } \\
\text { total, } \\
(\mu g / L)\end{array}$ \\
\hline \multicolumn{11}{|c|}{ Tanque Verde Creek at Tucson } \\
\hline $02-02-88$ & $<3.0$ & $<3.0$ & -- & $<5.0$ & $<5.0$ & $<5.0$ & $<3.0$ & $<3.0$ & $<3.0$ & $<3.0$ \\
\hline 01-06-92 & $<1.0$ & $<3.0$ & $<3.0$ & $<3.0$ & $<3.0$ & $<3.0$ & $<3.0$ & $<3.0$ & $<3.0$ & $<3.0$ \\
\hline \multicolumn{11}{|c|}{ Pantano Wash at Broadway Boulevard } \\
\hline $11-01-87$ & $<3.0$ & $<3.0$ & $-\cdots$ & $<5.0$ & $<5.0$ & $<5.0$ & $<3.0$ & $<3.0$ & $<3.0$ & $<3.0$ \\
\hline $07-24-90$ & $<1.0$ & $<3.0$ & $<3.0$ & $<5.0$ & $<5.0$ & $<5.0$ & $<3.0$ & $<3.0$ & $<3.0$ & $<3.0$ \\
\hline 02-13-92 & $<1.0$ & $<3.0$ & $<3.0$ & $<3.0$ & $<3.0$ & $<3.0$ & $<3.0$ & $<3.0$ & $<3.0$ & $<3.0$ \\
\hline \multicolumn{11}{|c|}{ Alamo Wash at Glenn Street } \\
\hline 09-04-87 & $<3.0$ & $<3.0$ & $\cdots$ & $<3.0$ & $<3.0$ & $<3.0$ & $<3.0$ & $<3.0$ & $<3.0$ & $<3.0$ \\
\hline $11-15-91$ & $<1.0$ & $<3.0$ & $<3.0$ & $<3.0$ & $<3.0$ & $<3.0$ & $<3.0$ & $<3.0$ & $<3.0$ & $<3.0$ \\
\hline 01-06-92 & $<1.0$ & $<3.0$ & $<3.0$ & $<3.0$ & $<3.0$ & $<3.0$ & $<3.0$ & $<3.0$ & $<3.0$ & $<3.0$ \\
\hline \multicolumn{11}{|c|}{ Rilito Creek at Dodge Boulevard } \\
\hline $07-30-88$ & $<3.0$ & $<3.0$ & -- & $<5.0$ & $<5.0$ & $<5.0$ & $<3.0$ & $<3.0$ & $<3.0$ & $<3.0$ \\
\hline 02-09-92 & $<1.0$ & $<3.0$ & $<3.0$ & $<3.0$ & $<3.0$ & $<3.0$ & $<3.0$ & $<3.0$ & $<3.0$ & $<3.0$ \\
\hline
\end{tabular}


Tabie 8. Analytical results of surface-water samples, Rillito Creek basin-Continued Organic carbon and oil and grease

\begin{tabular}{|c|c|c|c|c|}
\hline Date & $\begin{array}{c}\text { Discharge, } \\
\text { instantaneous, } \\
\left(\mathrm{ft}^{3} / \mathrm{s}\right)\end{array}$ & $\begin{array}{l}\text { Carbon organic, } \\
\text { dissolved } \\
\text { (mg/L as C) }\end{array}$ & $\begin{array}{l}\text { Carbon, organic total } \\
\text { (mg/Las C) }\end{array}$ & $\begin{array}{l}\text { Oil and grease, } \\
\text { total recoverable, } \\
\text { gravimetric (mg/L) }\end{array}$ \\
\hline \multicolumn{5}{|c|}{ Tanque Verde Creek at Tucson } \\
\hline $08-25-87$ & 23 & 10 & 9.3 & -- \\
\hline $02-02-88$ & 44 & 9.3 & 8.8 & $<1$ \\
\hline $08-20-88$ & 3,800 & 6.0 & 110 & 2 \\
\hline $08-01-89$ & 6 & 15 & 15 & $<1$ \\
\hline 08-03-89 & 69 & 13 & 87 & $<1$ \\
\hline $07-07-90$ & 450 & 19 & 210 & -- \\
\hline $07-20-90$ & 2,200 & --- & 53 & 1 \\
\hline $07-24-90$ & 4,400 & -- & 180 & $<1$ \\
\hline $12-28-90$ & 1,000 & -- & 240 & -- \\
\hline $01-05-91$ & 1,080 & --- & 74 & -- \\
\hline $01-06-92$ & 105 & -- & 13 & $<1$ \\
\hline $02-09-92$ & 120 & --- & 12 & $<1$ \\
\hline \multicolumn{5}{|c|}{ Pantano Wash at Broadway Boulevard } \\
\hline $11-01-87$ & 50 & --- & 64 & $<1$ \\
\hline $07-24-90$ & 3,710 & $-\cdots$ & 99 & $<1$ \\
\hline $07-24-90$ & 4,470 & 5.7 & 150 & $<1$ \\
\hline $02-13-92$ & 97 & --- & 30 & -- \\
\hline $12-29-92$ & 35 & 15 & $-\cdots$ & -- \\
\hline \multicolumn{5}{|c|}{ Alamo Wash at Glenn Street } \\
\hline $08-11-87$ & 230 & 18 & 69 & -- \\
\hline 09-04-87 & 458 & -.. & 29 & 2 \\
\hline $08-20-88$ & 4,000 & 7.2 & 93 & 3 \\
\hline $07-20-90$ & 629 & -- & 38 & $<1$ \\
\hline $07-24-90$ & 870 & -- & $-\cdots$ & $<1$ \\
\hline $10-27-91$ & 69 & $\cdots$ & 41 & 1 \\
\hline $11-15-91$ & 33 & --- & 20 & 1 \\
\hline $12-11-91$ & 49 & --- & 14 & $<1$ \\
\hline $01-06-92$ & 325 & --- & 15 & $<1$ \\
\hline \multicolumn{5}{|c|}{ Riltito Creek at Dodge Boulevard } \\
\hline $07-30-88$ & 5,300 & -- & $-\cdots$ & $<1$ \\
\hline $08-20-88$ & 5,900 & 7.3 & 210 & 2 \\
\hline $07-26-89$ & 3,940 & 14 & 190 & $<1$ \\
\hline $07-20-90$ & 4,800 & --- & -- & $<1$ \\
\hline $02-09-92$ & 95 & $\cdots$ & 19 & $<1$ \\
\hline $02-13-92$ & 4,500 & --- & 48 & $<1$ \\
\hline
\end{tabular}


Table 9. Analytical results of ground-water samples, Rillito Creek basin Properties and major ions

$\left[{ }^{\circ} \mathrm{C}\right.$, degrees Celsius; $\mu \mathrm{S} / \mathrm{cm}$, microsiemens per centimeter, $\mathrm{mg} / \mathrm{L}$, miligrams per liter; NTU nephelometric-turbidity units; $\mu \mathrm{g} / \mathrm{L}$, micrograms per liter; $\mathrm{pCi} / \mathrm{L}$, picocuries per liter, dashes indicate no data; $<$, less than]

\begin{tabular}{|c|c|c|c|c|c|c|c|c|c|c|c|}
\hline $\begin{array}{c}\text { Well } \\
\text { number } \\
(D-13-14)\end{array}$ & Date & $\begin{array}{l}\text { Tem- } \\
\text { pera- } \\
\text { ture, } \\
\text { water } \\
\left({ }^{\circ} \mathrm{C}\right)\end{array}$ & $\begin{array}{c}\text { Spe- } \\
\text { cific } \\
\text { con- } \\
\text { duct- } \\
\text { ance } \\
(\mu \mathrm{S} / \mathrm{cm})\end{array}$ & $\begin{array}{l}\text { Bicar- } \\
\text { bon- } \\
\text { ate, } \\
\text { lab } \\
\text { (mg/L } \\
\text { as } \\
\mathrm{HCO}_{3} \text { ) }\end{array}$ & $\begin{array}{l}\text { Oxygen, } \\
\text { dis- } \\
\text { solved } \\
\text { (mg/L) }\end{array}$ & $\begin{array}{c}\text { Tur- } \\
\text { bidity } \\
\text { (NTU) }\end{array}$ & $\begin{array}{c}\text { Solids, } \\
\text { residue } \\
\text { at } \\
180^{\circ} \mathrm{C}, \\
\text { dis- } \\
\text { solved } \\
\text { (mg/L) }\end{array}$ & $\begin{array}{c}\text { pH } \\
\text { (stan- } \\
\text { dard } \\
\text { units) }\end{array}$ & $\begin{array}{c}\text { Alka- } \\
\text { Ilnity, } \\
\text { lab } \\
\text { (mg/L } \\
\text { as } \\
\mathrm{CaCO}_{3} \text { ) }\end{array}$ & $\begin{array}{c}\text { Hard- } \\
\text { ness, } \\
\text { total } \\
\text { (mg/L } \\
\text { as } \\
\left.\mathrm{CaCO}_{3}\right)\end{array}$ & $\begin{array}{c}\text { Sillica, } \\
\text { dis- } \\
\text { solved } \\
\text { (mg/L } \\
\text { as } \\
\mathrm{SiO}_{2} \text { ) }\end{array}$ \\
\hline \multirow[t]{3}{*}{$34 d c c 2$} & $09-08-86$ & 24.0 & 605 & 267 & 6.5 & 0.5 & 370 & 7.2 & 219 & 190 & 38 \\
\hline & $06-16-87$ & 24.0 & 660 & -- & 6.1 & -- & $\cdots$ & 7.2 & $\cdots$ & --- & -- \\
\hline & $03-27-89$ & -.- & --- & 252 & --- & .4 & 317 &.-- & 207 & 150 & 36 \\
\hline \multirow[t]{3}{*}{ 34aaal } & 09-09-86 & 19.0 & 267 & 113 & 8.0 & .2 & 183 & 7.4 & 93 & 59 & 24 \\
\hline & $06-16-87$ & 19.0 & 240 & --- & 9.3 & --- & -- & 7.4 & --- & --- & --- \\
\hline & $03-29-89$ & -- & -- & 121 & --- & .1 & 166 & -.- & 99 & 62 & 25 \\
\hline \multirow[t]{3}{*}{$27 d d c 3$} & $08-27-86$ & 21.0 & 275 & 138 & 7.2 & .6 & 187 & 7.7 & 113 & 82 & 27 \\
\hline & $06-15-87$ & 19.0 & 295 & --- & 5.9 & -- & -- & 7.5 & --- & $\cdots$ & $\cdots$ \\
\hline & $03-28-89$ & -- & --- & 154 & --- & .1 & 202 & -- & 126 & 92 & 27 \\
\hline \multirow[t]{2}{*}{$26 \mathrm{cdd}$} & 09-09-86 & 17.5 & 225 & 105 & 5.1 & .8 & 136 & 7.0 & 86 & 81 & 24 \\
\hline & $06-16-87$ & 17.0 & 220 & --- & 5.7 & -- & --- & 7.1 & --- & --- & -- \\
\hline \multirow[t]{3}{*}{$27 \mathrm{cda}$} & $08-25-86$ & 20.5 & 360 & 170 & 4.1 & .2 & 241 & 7.8 & 139 & 95 & 28 \\
\hline & $03-30-89$ & -- & -.- & 176 & --- & .1 & 218 & --- & 144 & 95 & 29 \\
\hline & $11-03-89$ & 20.0 & 375 & -- & 4.8 & --- & --- & 7.6 & $-\cdots$ & --- & -- \\
\hline \multirow[t]{3}{*}{ 26dda2 } & $08-26-86$ & 20.5 & 405 & 205 & 3.7 & .4 & 245 & 7.3 & 168 & 170 & 20 \\
\hline & $03-29-89$ & -- & -- & 141 & -- & .5 & 190 & -- & 116 & 130 & 20 \\
\hline & $11-03-89$ & 21.5 & 430 & --- & 6.0 & -- & -.. & 6.7 & $\ldots$ & -- & -- \\
\hline \multirow[t]{3}{*}{$26 \mathrm{dac} 2$} & $08-26-86$ & 21.0 & 255 & 124 & 2.6 & 1.9 & 137 & 6.9 & 102 & 99 & 19 \\
\hline & $06-16-87$ & 19.0 & 320 & -- & 4.4 & --- & $-\ldots$ & 7.3 & -- & -.- & -- \\
\hline & $03-29-89$ & -.- & --- & 165 & --- & .2 & 216 &.- & 135 & 150 & 19 \\
\hline \multirow[t]{2}{*}{$28 \mathrm{dad}$} & $08-25-86$ & 18.0 & 235 & 143 & 10.4 & .3 & 187 & 7.9 & 117 & 110 & 28 \\
\hline & $03-29-89$ & $\cdots$ & -- & 129 & --- & .1 & 152 & --- & 106 & 92 & 28 \\
\hline \multirow[t]{3}{*}{ 27ada2 } & $08-27-86$ & 24.0 & 603 & 233 & 6.2 & .2 & 363 & 7.6 & 191 & 190 & 39 \\
\hline & $06-15-87$ & 25.0 & 685 & --- & 6.7 & --- & $-\ldots$ & 7.3 & --- & --- &.-- \\
\hline & $03-29-89$ & -- & -- & 216 & --- & .1 & 308 & --- & 177 & 170 & 40 \\
\hline \multirow[t]{4}{*}{$27 \mathrm{bdb} 4$} & $08-28-86$ & 16.5 & 317 & 152 & 3.1 & .3 & 197 & 6.9 & 125 & 140 & 17 \\
\hline & $09-08-86$ & 17.5 & 215 & 101 & 3.9 & 1.8 & 132 & 7.0 & 83 & 90 & 18 \\
\hline & $06-15-87$ & 16.0 & 245 & --- & 3.6 & --- & --- & 7.0 & --- & --- & -- \\
\hline & $03-27-89$ & -- & $\ldots$ & 243 & --- & 90 & 315 & --- & 199 & 140 & 30 \\
\hline \multirow[t]{3}{*}{$26 \mathrm{bbb}$} & $09-08-86$ & 25.0 & 720 & 152 & 7.2 & .6 & 480 & 7.6 & 125 & 250 & 43 \\
\hline & $06-15-87$ & 25.0 & 690 & --- & 4.7 & -- & --- & 7.7 & $\ldots$ & $\ldots$ & --- \\
\hline & $03-27-89$ & --- & --- & 139 & --- & 3.8 & 689 &.- & 114 & 390 & 43 \\
\hline $26 \mathrm{dcb} 2$ & $03-28-89$ & 17.5 & 230 & 111 & 4.6 & 25 & 146 & 6.9 & 91 & 90 & 23 \\
\hline $26 \mathrm{cbb} 3$ & $03-28-89$ & 21.5 & 305 & 136 & 2.7 & 3.5 & 187 & 6.7 & 111 & 120 & 26 \\
\hline $26 \mathrm{cbb} 2$ & $03-28-89$ & 20.0 & 470 & 180 & 5.8 & .6 & 248 & 7.4 & 148 & 150 & 33 \\
\hline
\end{tabular}


Table 9. Analytical results of ground-water samples, Rillito Creek basin-Continued Properties and major ions-Continued

\begin{tabular}{|c|c|c|c|c|c|c|c|c|c|}
\hline $\begin{array}{c}\text { Weil } \\
\text { number } \\
\text { (D-13-14) }\end{array}$ & Date & $\begin{array}{c}\text { Cal- } \\
\text { clum, } \\
\text { dissoived } \\
\text { (mg/L } \\
\text { as Ca) }\end{array}$ & $\begin{array}{c}\text { Magnesium, } \\
\text { dissoived } \\
\text { (mg/L } \\
\text { as Mg) }\end{array}$ & $\begin{array}{l}\text { Potas- } \\
\text { sium, } \\
\text { dissolved } \\
\text { (mg/L } \\
\text { as K) }\end{array}$ & $\begin{array}{l}\text { Sodium, } \\
\text { dissolved } \\
\text { (mg/L } \\
\text { as } \mathrm{Na} \text { ) }\end{array}$ & $\begin{array}{c}\text { Sodium } \\
\text { adsorp- } \\
\text { tion } \\
\text { ratio }\end{array}$ & $\begin{array}{c}\text { Chloride, } \\
\text { dissoived } \\
\text { (mg/L } \\
\text { as Cl) }\end{array}$ & $\begin{array}{c}\text { Sulfate, } \\
\text { dissolved } \\
\text { (mg/L } \\
\left.\text { as } \mathrm{SO}_{4}\right)\end{array}$ & $\begin{array}{l}\text { Fluoride, } \\
\text { dissolved } \\
\text { (mg/L } \\
\text { as F) }\end{array}$ \\
\hline \multirow[t]{2}{*}{$34 \mathrm{dcc} 2$} & $09-08-86$ & 64 & 6.4 & 2.1 & 55 & 2 & 16 & 42 & 0.2 \\
\hline & $03-27-89$ & 51 & 5.4 & 2.0 & 46 & 2 & 10 & 28 & .1 \\
\hline \multirow[t]{2}{*}{ 34aaa1 } & $09-09-86$ & 22 & 1.0 & 1.0 & 29 & 2 & 10 & 20 & .3 \\
\hline & 03-29-89 & 23 & 1.1 & .9 & 31 & 2 & 9.0 & 17 & .3 \\
\hline \multirow[t]{2}{*}{$27 \mathrm{ddc} 3$} & $08-27-86$ & 31 & 1.1 & 1.3 & 25 & 1 & 4.2 & 16 & .2 \\
\hline & $03-28-89$ & 35 & 1.1 & 1.4 & 29 & 1 & 4.9 & 22 & .2 \\
\hline $26 \mathrm{cdd}$ & $09-09-86$ & 29 & 2.1 & 1.2 & 12 & .6 & 3.5 & 16 & .3 \\
\hline \multirow[t]{2}{*}{$27 \mathrm{cda}$} & $08-25-86$ & 36 & 1.2 & 1.5 & 36 & 2 & 8.7 & 27 & .5 \\
\hline & $03-30-89$ & 36 & 1.3 & 1.2 & 39 & 2 & 6.8 & 22 & .5 \\
\hline \multirow[t]{2}{*}{ 26dda2 } & $08-26-86$ & 59 & 6.3 & 2.3 & 14 & .5 & 10 & 22 & .3 \\
\hline & $03-29-89$ & 43 & 4.7 & 1.9 & 13 & .5 & 8.8 & 33 & .2 \\
\hline \multirow[t]{2}{*}{26 dac2 } & $08-26-86$ & 34 & 3.4 & 1.9 & 9.6 & .4 & 4.2 & 12 & .2 \\
\hline & $03-29-89$ & 51 & 5.0 & 2.0 & 15 & .5 & 12 & 27 & .2 \\
\hline \multirow[t]{2}{*}{28 dad } & $08-26-86$ & 40 & 1.9 & 1.4 & 13 & .5 & 3.2 & 17 & .2 \\
\hline & $03-29-89$ & 34 & 1.7 & 1.0 & 13 & .6 & 2.0 & 10 & .2 \\
\hline \multirow[t]{2}{*}{ 27ada2 } & $08-27-86$ & 59 & 10 & 2.5 & 43 & 1 & 43 & 23 & .4 \\
\hline & $03-29-89$ & 51 & 9.3 & 2.3 & 36 & 1 & 29 & 16 & .4 \\
\hline \multirow[t]{3}{*}{ 27bdb4 } & $08-28-86$ & 46 & 5.0 & 2.1 & 9.0 & .3 & 3.0 & 25 & .2 \\
\hline & $09-08-86$ & 31 & 3.1 & 1.9 & 7.2 & .3 & 2.2 & 15 & .3 \\
\hline & $03-27-89$ & 48 & 5.2 & 2.2 & 51 & 2 & 18 & 20 & .5 \\
\hline \multirow[t]{2}{*}{$26 \mathrm{bbb}$} & $09-08-86$ & 80 & 13 & 2.5 & 36 & 1 & 97 & 13 & .6 \\
\hline & $03-27-89$ & 120 & 21 & 3.1 & 42 & .9 & 180 & 17 & .3 \\
\hline $26 \mathrm{dcb} 2$ & $03-28-89$ & 32 & 2.5 & 1.0 & 8.8 & .4 & 3.6 & 13 & .2 \\
\hline $26 \mathrm{cbb} 3$ & $03-28-89$ & 44 & 3.4 & 1.1 & 9.2 & .4 & 3.4 & 25 & .2 \\
\hline $26 \mathrm{cbb} 2$ & $03-28-89$ & 53 & 4.3 & 1.5 & 21 & .7 & 9.5 & 28 & .2 \\
\hline
\end{tabular}


Table 9. Analytical results of ground-water samples, Rillito Creek basin Nutrients

\begin{tabular}{|c|c|c|c|c|c|c|c|c|}
\hline $\begin{array}{c}\text { Well } \\
\text { number } \\
\text { (D-13-14) }\end{array}$ & Date & $\begin{array}{c}\text { Phosphate, } \\
\text { total } \\
(\mathrm{mg} / \mathrm{L} \text { as } \\
\left.\mathrm{PO}_{4}\right)\end{array}$ & $\begin{array}{l}\text { Phosphate, } \\
\text { ortho, } \\
\text { dissolved } \\
\text { (mg/L as } \\
\mathrm{PO}_{4} \text { ) }\end{array}$ & $\begin{array}{c}\text { Phosphorus, } \\
\text { total } \\
\text { (mg/L as P) }\end{array}$ & $\begin{array}{l}\text { Phosphorus, } \\
\text { dissolved } \\
\text { (mg/L as P) }\end{array}$ & $\begin{array}{l}\text { Phos- } \\
\text { phorus, } \\
\text { ortho } \\
\text { dlssolved } \\
\text { (mg/L as P) }\end{array}$ & $\begin{array}{l}\text { Nitrogen, } \\
\text { total } \\
\text { (mg/L as } N \text { ) }\end{array}$ & $\begin{array}{c}\text { Nitrogen, } \\
\text { organic } \\
\text { total } \\
\text { (mg/L as } N \text { ) }\end{array}$ \\
\hline \multirow[t]{2}{*}{$34 \mathrm{dcc} 2$} & $09-08-86$ & 0.06 & $\ldots$ & 0.02 & 0.02 & $<0.01$ & 3.9 & 0.37 \\
\hline & $03-27-89$ & .03 & 0.03 & .02 & .02 & .01 & 2.6 & $-\cdots$ \\
\hline 34aaal & $09-09-86$ & .06 & .06 & .02 & .02 & .02 & 1.3 & -- \\
\hline \multirow[t]{2}{*}{$27 \mathrm{ddc} 3$} & $08-27-86$ & .03 & $\ldots$ & .03 & .03 & $<.01$ & 3.4 & 2.1 \\
\hline & $03-28-89$ & .09 & --- & $<.01$ & $<.01$ & $<.01$ & -- & --- \\
\hline $26 \mathrm{cdd}$ & $09-09-86$ & .09 & .09 & .04 & .04 & .03 & .80 & --- \\
\hline $27 \mathrm{cda}$ & $08-25-86$ & --- & $\ldots$ & .02 & .01 & $<.01$ & --- & --- \\
\hline 26dda2 & $08-26-86$ & .12 & .06 & .04 & .03 & .02 & .70 & .16 \\
\hline 26dac2 & $08-26-86$ & .12 & .06 & .07 & .03 & .02 & --- & -- \\
\hline 28dad & $08-25-86$ & --- & -- & .02 & .01 & $<.01$ & -- & $-\cdots$ \\
\hline \multirow[t]{3}{*}{ 27ada2 } & $08-27-86$ & --- & --- & .02 & .01 & $<.01$ & 9.7 & .46 \\
\hline & $06-15-87$ & --- & $\cdots$ & .03 & .02 & $<.01$ & 8.6 & .69 \\
\hline & $03-29-89$ & .06 & --- & $<.01$ & $<.01$ & $<.01$ & 4.8 & .36 \\
\hline \multirow[t]{2}{*}{$27 \mathrm{bdb} 4$} & $08-28-86$ & --- & --- & --- & --- & --- & --- & $-\cdots$ \\
\hline & $09-08-86$ & .18 & .15 & .07 & .06 & .05 & 1.1 & .48 \\
\hline \multirow[t]{3}{*}{$26 \mathrm{bbb}$} & $09-08-86$ & --. & --- & .01 & $<.01$ & $<.01$ & 19 & 1.1 \\
\hline & $06-15-87$ & --- & --- & .03 & .02 & $<.01$ & 17 & 1.8 \\
\hline & $03-27-89$ & --- & --- & $<.01$ & $<.01$ & $<.01$ & 25 & .27 \\
\hline $26 \mathrm{dcb} 2$ & $03-28-89$ & .12 & .06 & .12 & .04 & .02 & --- & $\cdots$ \\
\hline $26 \mathrm{cbb3}$ & $03-28-89$ & .06 & --- & .03 & .01 & $<.01$ & 2.3 & .45 \\
\hline $26 \mathrm{cbb} 2$ & $03-28-89$ & $\ldots$ & -.- & .01 & .01 & $<.01$ & 2.1 & .39 \\
\hline
\end{tabular}


Table 9. Analytical results of ground-water samples, Rillito Creek basin-Continued Nutrients-Continued

\begin{tabular}{|c|c|c|c|c|c|c|c|c|}
\hline $\begin{array}{c}\text { Well } \\
\text { number } \\
(D-13-14)\end{array}$ & Date & $\begin{array}{l}\text { Nitrogen, } \\
\text { ammonia, } \\
\text { dissolved } \\
\text { (mg/L as N) }\end{array}$ & $\begin{array}{c}\text { Nitrogen, } \\
\text { ammonia } \\
\text { total } \\
\text { (mg/L as } N \text { ) }\end{array}$ & $\begin{array}{l}\text { Nitrogen, } \\
\text { nitrite, } \\
\text { total } \\
\text { (mg/L as N) }\end{array}$ & $\begin{array}{l}\text { Nitrogen, } \\
\text { nitrate } \\
\text { total } \\
\text { (mg/L as N) }\end{array}$ & $\begin{array}{c}\text { Nitrogen, } \\
\text { ammonia } \\
\text { plus organic, } \\
\text { total } \\
\text { (mg/L as N) }\end{array}$ & $\begin{array}{c}\text { Nitrogen, } \\
\mathrm{NO}_{2}+\mathrm{NO}_{3}, \\
\text { total } \\
\text { (mg/Las } \mathrm{N} \text { ) }\end{array}$ & $\begin{array}{l}\text { Nitrogen, } \\
\mathrm{NO}_{2}+\mathrm{NO}_{3}, \\
\text { dissolved } \\
\text { (mg/L as } \mathrm{N} \text { ) }\end{array}$ \\
\hline \multirow[t]{2}{*}{$34 \mathrm{dcc} 2$} & $09-08-86$ & 0.02 & 0.03 & 0.01 & 3.49 & 0.40 & 3.50 & 3.40 \\
\hline & $03-27-89$ & $<.01$ & $<.01$ & $<.01$ & --- & .20 & 2.40 & 2.40 \\
\hline 34aaal & $09-09-86$ & $<.01$ & $<.01$ & $<.01$ & -- & .40 & .90 & .95 \\
\hline \multirow[t]{2}{*}{$27 \mathrm{ddc} 3$} & $08-27-86$ & $<.01$ & .05 & $<.01$ & --- & 2.2 & 1.20 & 1.20 \\
\hline & 03-28-89 & $<.01$ & .05 & $<.01$ & -.- & $<.20$ & 1.40 & 1.40 \\
\hline $26 \mathrm{cdd}$ & $09-09-86$ & $<.01$ & $<.01$ & $<.01$ & --- & .20 & .60 & .92 \\
\hline $27 \mathrm{cda}$ & $08-25-86$ & .02 & $<.01$ & $<.01$ & --. & $<.20$ & 1.60 & 1.50 \\
\hline 26dda2 & $08-26-86$ & $<.01$ & .04 & $<.01$ & --- & .20 & .50 & .43 \\
\hline 26 dac2 & $08-26-86$ & $<.01$ & .04 & .02 & .48 & $<.20$ & .50 & .52 \\
\hline 28dad & $08-25-86$ & .01 & $<.01$ & $<.01$ & $\ldots$ & $<.20$ & 1.10 & 1.10 \\
\hline \multirow[t]{3}{*}{$27 \mathrm{ada} 2$} & $08-27-86$ & $<.01$ & .04 & $<.01$ & --- & .50 & 9.20 & 6.20 \\
\hline & $06-15-87$ & .01 & .01 & $<.01$ & --- & .70 & 7.90 & 7.30 \\
\hline & 03-29-89 & $<.01$ & .04 & $<.01$ & --- & .40 & 4.40 & 4.50 \\
\hline \multirow[t]{2}{*}{$27 \mathrm{bdb} 4$} & $08-28-86$ & --- & --- & --- & --- & --- & --- & --- \\
\hline & $09-08-86$ & .01 & .02 & .01 & .59 & .50 & .60 & .61 \\
\hline \multirow[t]{3}{*}{$26 \mathrm{bbb}$} & $09-08-86$ & .05 & .04 & .01 & 18.0 & 1.1 & 18.0 & 16.0 \\
\hline & $06-15-87$ & .02 & .01 & $<.01$ & --- & 1.8 & 15.0 & 15.0 \\
\hline & $03-27-89$ & .04 & .03 & $<.01$ & $-\cdots$ & .3 & 25.0 & 23.0 \\
\hline $26 \mathrm{dcb} 2$ & $03-28-89$ & $<.01$ & .04 & $<.01$ & --- & $<.20$ & 1.00 & 1.00 \\
\hline $26 \mathrm{cbb} 3$ & $03-28-89$ & $<.01$ & .05 & $<.01$ & --- & .50 & 1.80 & 1.90 \\
\hline $26 \mathrm{cbb} 2$ & $03-28-89$ & .01 & .01 & $<.01$ & -- & .40 & 1.70 & 1.70 \\
\hline
\end{tabular}


Table 9. Analytical results of ground-water samples, Rillito Creek basin Trace elements

\begin{tabular}{|c|c|c|c|c|c|c|c|c|c|c|}
\hline $\begin{array}{c}\text { Well } \\
\text { number } \\
\text { (D-13-14) }\end{array}$ & Date & $\begin{array}{c}\text { Barium, } \\
\text { total } \\
\text { recov- } \\
\text { erable } \\
(\mu g / L \\
\text { as Ba) }\end{array}$ & $\begin{array}{c}\text { Zinc, } \\
\text { total } \\
\text { recov- } \\
\text { erable } \\
\text { ( } \mu \mathrm{g} / \mathrm{L} \\
\text { as } \mathrm{Zn})\end{array}$ & $\begin{array}{c}\text { Selenium, } \\
\text { total } \\
(\mu \mathrm{g} / \mathrm{L} \\
\text { as Se) }\end{array}$ & $\begin{array}{c}\text { Molyb- } \\
\text { denum, } \\
\text { total } \\
\text { recov- } \\
\text { erable } \\
(\mu g / L \\
\text { as Mo) }\end{array}$ & $\begin{array}{c}\text { Arsenic, } \\
\text { total } \\
(\mu g / L \\
\text { as As })\end{array}$ & $\begin{array}{c}\text { Copper, } \\
\text { total } \\
\text { recov- } \\
\text { erable } \\
(\mu g / L \\
\text { as Cu) }\end{array}$ & $\begin{array}{c}\text { Mercury, } \\
\text { total } \\
\text { recov- } \\
\text { erable } \\
(\mu g / L \\
\text { as } \mathrm{Hg})\end{array}$ & $\begin{array}{c}\text { Chro- } \\
\text { mlum, } \\
\text { total } \\
\text { recov- } \\
\text { erable } \\
\text { ( } \mu \text { g/L } \\
\text { as } \mathrm{Cr} \text { ) }\end{array}$ & $\begin{array}{c}\text { Silver, } \\
\text { total } \\
\text { recov- } \\
\text { erable } \\
(\mu g / L \\
\text { as Ag) }\end{array}$ \\
\hline $34 \mathrm{dcc} 2$ & $09-08-86$ & 100 & 40 & $<1$ & 1 & $<1$ & 3 & $<0.10$ & $<10$ & $<1$ \\
\hline \multirow[t]{3}{*}{ 34aaa1 } & $09-09-86$ & $<100$ & 100 & $<1$ & 2 & 4 & 33 & $<.10$ & 10 & $<1$ \\
\hline & $06-16-87$ & $<100$ & $<10$ & $<1$ & $<1$ & 3 & 2 & $<.10$ & 10 & $<1$ \\
\hline & $03-29-89$ & 100 & $<10$ & $<1$ & 2 & 4 & 1 & $<.10$ & 2 & $<1$ \\
\hline $27 \mathrm{ddc} 3$ & $08-27-86$ & $<100$ & 30 & $<1$ & 2 & $<1$ & 3 & $<.10$ & $<10$ & $<1$ \\
\hline $26 \mathrm{cdd}$ & $09-09-86$ & $<100$ & 10 & $<1$ & 1 & $<1$ & 3 & .10 & 10 & $<1$ \\
\hline $27 \mathrm{cda}$ & $08-25-86$ & $<100$ & 30 & $<1$ & 5 & 3 & 3 & $<.10$ & $<10$ & $<1$ \\
\hline 26dda2 & $08-26-86$ & 100 & 90 & $<1$ & $<1$ & $<1$ & 6 & $<.10$ & 10 & $<1$ \\
\hline 26 dac2 & $08-26-86$ & 100 & 60 & $<1$ & 3 & $<1$ & 7 & $<.10$ & $<10$ & $<1$ \\
\hline 28dad & $08-25-86$ & $<100$ & 60 & $<1$ & 4 & $<1$ & 4 & .10 & $<10$ & $<1$ \\
\hline 27ada2 & $08-27-86$ & 100 & 20 & $<1$ & 2 & 3 & 6 & $<.10$ & $<10$ & $<1$ \\
\hline \multirow[t]{2}{*}{ 27bdb4 } & $08-28-86$ & --- & --- & --. & --- & --. & --. & --- & $-\ldots$ & --- \\
\hline & $09-08-86$ & $<100$ & $<10$ & $<1$ & 2 & 1 & 6 & $<.10$ & $<10$ & $<1$ \\
\hline \multirow[t]{2}{*}{$26 \mathrm{bbb}$} & $09-08-86$ & 200 & 50 & $<1$ & 1 & 2 & 5 & $<.10$ & $<10$ & $<1$ \\
\hline & $03-27-89$ & 400 & 40 & 2 & 3 & 2 & 5 & $<.10$ & 3 & $<1$ \\
\hline $26 \mathrm{dcb} 2$ & $03-28-89$ & 100 & 20 & $<1$ & 2 & 2 & 6 & $<.10$ & 4 & $<1$ \\
\hline $26 \mathrm{cbb} 3$ & $03-28-89$ & 200 & $<10$ & $<1$ & 2 & 2 & 1 & $<.10$ & 2 & 1 \\
\hline $26 \mathrm{cbb} 2$ & $03-28-89$ & $<100$ & 40 & $<1$ & 2 & 1 & 1 & $<.10$ & 3 & $<1$ \\
\hline
\end{tabular}


Table 9. Analytical results of ground-water samples, Rillito Creek basin-Continued Trace elements-Continued

\begin{tabular}{|c|c|c|c|c|c|c|c|c|c|c|}
\hline $\begin{array}{c}\text { Well } \\
\text { number } \\
(D-13-14)\end{array}$ & Date & $\begin{array}{l}\text { Nickel, } \\
\text { total } \\
\text { recov- } \\
\text { erable } \\
(\mu \mathrm{g} / \mathrm{L} \\
\text { as } \mathrm{Ni})\end{array}$ & $\begin{array}{c}\text { Manga- } \\
\text { nese, } \\
\text { total } \\
\text { recov- } \\
\text { erable } \\
(\mu g / L \\
\text { as } \mathrm{Mn})\end{array}$ & $\begin{array}{c}\text { Iron, } \\
\text { total } \\
(\mu g / L \\
\text { as } \mathrm{Fe})\end{array}$ & $\begin{array}{c}\text { Beryl- } \\
\text { lium, } \\
\text { total } \\
\text { recov- } \\
\text { erable } \\
(\mu \mathrm{g} / \mathrm{L} \\
\text { as } \mathrm{Be})\end{array}$ & $\begin{array}{l}\text { Lithlum, } \\
\text { total } \\
\text { recov- } \\
\text { erable } \\
\text { ( } \mu \mathrm{g} / \mathrm{L} \\
\text { as L) }\end{array}$ & $\begin{array}{c}\text { Alum- } \\
\text { inum, } \\
\text { total } \\
\text { recov- } \\
\text { erable } \\
(\mu g / L \\
\text { as Al) }\end{array}$ & $\begin{array}{c}\text { Cobalt, } \\
\text { total } \\
\text { recov- } \\
\text { erable } \\
(\mu g / L \\
\text { as Co) }\end{array}$ & $\begin{array}{c}\text { Cadmium, } \\
\text { total } \\
\text { recov- } \\
\text { erable } \\
(\mu g / L \\
\text { as Cd) }\end{array}$ & $\begin{array}{c}\text { Lead, } \\
\text { total } \\
\text { recov- } \\
\text { erable } \\
(\mu g / L \\
\text { as Pb) }\end{array}$ \\
\hline $34 \mathrm{dcc} 2$ & $09-08-86$ & 3 & 20 & 380 & $<10$ & $<10$ & -- & $<1$ & $<1$ & $<5$ \\
\hline \multirow[t]{3}{*}{ 34aaa1 } & $09-09-86$ & 9 & 20 & 160 & $<10$ & 30 & 80 & $<1$ & $<1$ & 38 \\
\hline & $06-16-87$ & $<1$ & $<10$ & $<10$ & $<10$ & 20 & $<10$ & $<1$ & $<1$ & $<5$ \\
\hline & 03-29-89 & $<1$ & $<10$ & 50 & $<10$ & 30 & $<10$ & $<1$ & $<1$ & $<5$ \\
\hline $27 \mathrm{ddc} 3$ & $08-27-86$ & 4 & $<10$ & 10 & $<10$ & 30 & $<10$ & $<1$ & $<1$ & $<5$ \\
\hline $26 \mathrm{cdd}$ & $09-09-86$ & 5 & $<10$ & 110 & $<10$ & $<10$ & 80 & $<1$ & $<1$ & $<5$ \\
\hline $27 \mathrm{cda}$ & $08-25-86$ & 1 & $<10$ & 20 & $<10$ & 60 & $<10$ & $<1$ & $<1$ & $<5$ \\
\hline 26dda2 & $08-26-86$ & 6 & $<10$ & 70 & $<10$ & $<10$ & $<10$ & $<1$ & $<1$ & 11 \\
\hline 26dac2 & $08-26-86$ & 2 & 40 & 960 & $<10$ & $<10$ & 10 & $<1$ & $<1$ & $<5$ \\
\hline 28dad & $08-25-86$ & 2 & $<10$ & 80 & $<10$ & 10 & $<10$ & $<1$ & $<1$ & $<5$ \\
\hline 27ada2 & $08-27-86$ & 4 & 10 & 40 & $<10$ & 30 & $<10$ & $<1$ & $<1$ & $<5$ \\
\hline $27 \mathrm{bdb} 4$ & 09-08-86 & 2 & 10 & 730 & $<10$ & $<10$ & -- & 1 & --- & --- \\
\hline \multirow[t]{2}{*}{$26 \mathrm{bbb}$} & $09-08-86$ & 4 & 180 & 1,200 & $<10$ & 30 & -- & 3 & $<1$ & $<5$ \\
\hline & $03-27-89$ & 4 & 180 & 2,300 & $<10$ & 30 & 60 & $<1$ & $<1$ & $<5$ \\
\hline $26 \mathrm{dcb} 2$ & $03-28-89$ & 2 & 160 & 3,400 & $<10$ & $<10$ & 2,200 & 9 & $<1$ & $<5$ \\
\hline $26 \mathrm{cbb} 3$ & 03-28-89 & $<1$ & 20 & 840 & $<10$ & 10 & $<10$ & $<1$ & $<1$ & $<5$ \\
\hline $26 \mathrm{cbb} 2$ & $03-28-89$ & $<1$ & 30 & 2,600 & $<10$ & $<10$ & 120 & 2 & $<1$ & $<5$ \\
\hline
\end{tabular}


Table 9. Analytical results of ground-water samples, Rillito Creek basin-Continued Radionuclides

\begin{tabular}{|c|c|c|c|c|c|}
\hline $\begin{array}{c}\text { Well } \\
\text { number } \\
(D-13-14)\end{array}$ & Date & $\begin{array}{c}\text { Gross alpha, } \\
\text { dissoived } \\
\text { ( } \mu \text { g/L as U-Nat) }\end{array}$ & $\begin{array}{c}\text { Gross beta, } \\
\text { dissolved } \\
\text { (pCi/L as Sr-90/Y-90) }\end{array}$ & $\begin{array}{c}\text { Gross beta, } \\
\text { dissoived } \\
\text { (pCi/L as Cs-137) }\end{array}$ & $\begin{array}{l}\text { Radon-222, } \\
\text { total (pCi/L) }\end{array}$ \\
\hline $34 \mathrm{dcc} 2$ & $03-27-89$ & 3.5 & 3.5 & 4.6 & 140 \\
\hline $34 \mathrm{aaa1}$ & 03-29-89 & 3.3 & 2.2 & 2.9 & 400 \\
\hline $27 \mathrm{ddc} 3$ & $03-28-89$ & 9.2 & .8 & 1.0 & 470 \\
\hline $27 \mathrm{cda}$ & $03-30-89$ & 9.0 & 3.8 & 5.3 & 690 \\
\hline 26dda 2 & 03-29-89 & 1.0 & 2.0 & 2.3 & 210 \\
\hline $26 \mathrm{dac} 2$ & $03-29-89$ & 1.3 & 3.2 & 4.2 & 170 \\
\hline 28dad & 03-29-89 & 3.5 & 1.8 & 2.4 & 270 \\
\hline 27 ada2 & 03-29-89 & 4.5 & 3.5 & 4.6 & 450 \\
\hline $27 \mathrm{bdb} 4$ & $03-27-89$ & 6.8 & 4.3 & 5.7 & 150 \\
\hline $26 \mathrm{bbb}$ & 03-27-89 & 1.9 & 4.6 & 6.7 & 120 \\
\hline $26 \mathrm{dcb} 2$ & $03-28-89$ & $<.6$ & .6 & .7 & 500 \\
\hline $26 \mathrm{cbb} 2$ & $03-28-89$ & 2.3 & 2.0 & 2.7 & 440 \\
\hline $26 \mathrm{cbb3}$ & $03-28-89$ & .6 & 1.2 & 1.6 & 480 \\
\hline
\end{tabular}

Organochlorine pesticides

[DDD, dichlorodiphenyldichloroethane; DDE, dichlorodiphenylethylene; DDT, dichlorodiphenyltrichloroethane; PCB, polychlorinated biphenyl]

\begin{tabular}{|c|c|c|c|c|c|c|c|c|c|}
\hline $\begin{array}{l}\text { Well number } \\
(D-13-14)\end{array}$ & Date & $\begin{array}{c}\text { Perthane, } \\
\text { total } \\
(\mu \mathrm{g} / \mathrm{L})\end{array}$ & $\begin{array}{c}\text { Endosulfate, } \\
\text { total } \\
(\mu g / L)\end{array}$ & $\begin{array}{l}\text { Aldrin, } \\
\text { total } \\
(\mu \mathrm{g} / \mathrm{L})\end{array}$ & $\begin{array}{c}\text { Chlordane, } \\
\text { total } \\
(\mu \mathrm{g} / \mathrm{L})\end{array}$ & $\begin{array}{c}\text { DDD, } \\
\text { total } \\
(\mu g / L)\end{array}$ & $\begin{array}{c}\text { DDE, } \\
\text { total } \\
(\mu g / L)\end{array}$ & $\begin{array}{c}\text { DDT, } \\
\text { total } \\
(\mu g / L)\end{array}$ & $\begin{array}{c}\text { Dieldrin, } \\
\text { total } \\
(\mu g / L)\end{array}$ \\
\hline $34 \mathrm{dcc} 2$ & $09-08-86$ & $<0.1$ & $<0.010$ & $<0.010$ & $<0.1$ & $<0.010$ & $<0.010$ & $<0.010$ & $<0.010$ \\
\hline 34aaa1 & $09-09-86$ & $<.1$ & $<.010$ & $<.010$ & $<.1$ & $<.010$ & $<.010$ & $<.010$ & $<.010$ \\
\hline $27 \mathrm{ddc} 3$ & $08-27-86$ & $<1$ & $<.010$ & $<.010$ & $<1$ & $<.010$ & $<.010$ & $<.010$ & $<.010$ \\
\hline $26 \mathrm{cdd}$ & 09-09-86 & $<1$ & $<.010$ & $<.010$ & $<.1$ & $<.010$ & $<.010$ & $<.010$ & $<.010$ \\
\hline $27 \mathrm{cda}$ & $08-25-86$ & $<1$ & $<.010$ & $<.010$ & $<1$ & $<.010$ & $<.010$ & $<.010$ & $<.010$ \\
\hline 26dda2 & $08-26-86$ & $<.1$ & $<.010$ & $<.010$ & $<.1$ & $<.010$ & $<.010$ & $<.010$ & $<.010$ \\
\hline $26 \mathrm{dac} 2$ & $08-26-86$ & $<.1$ & $<.010$ & $<.010$ & $<1$ & $<.010$ & $<.010$ & $<.010$ & $<.010$ \\
\hline $28 \mathrm{dad}$ & $08-25-86$ & $<1$ & $<.010$ & $<.010$ & $<.1$ & $<.010$ & $<.010$ & $<.010$ & $<.010$ \\
\hline 27 ada2 & $08-27-86$ & $<.1$ & $<.010$ & $<.010$ & $<.1$ & $<.010$ & $<.010$ & $<.010$ & $<.010$ \\
\hline $27 \mathrm{bdb} 4$ & $09-08-86$ & $<1$ & $<.010$ & $<.010$ & $<1$ & $<.010$ & $<.010$ & $<.010$ & $<.010$ \\
\hline $26 \mathrm{bbb}$ & $09-08-86$ & $<1$ & $<.010$ & $<.010$ & $<.1$ & $<.010$ & $<.010$ & $<.010$ & $<.010$ \\
\hline
\end{tabular}

\begin{tabular}{|c|c|c|c|c|c|c|c|c|c|c|}
\hline $\begin{array}{c}\text { Well } \\
\text { number } \\
(D-13-14)\end{array}$ & Date & $\begin{array}{c}\text { Endrin, } \\
\text { total } \\
(\mu g / L)\end{array}$ & $\begin{array}{c}\text { Hepte- } \\
\text { chlor, } \\
\text { total } \\
(\mu g / L)\end{array}$ & $\begin{array}{c}\text { Hepta- } \\
\text { chlor } \\
\text { epoxide, } \\
\text { total } \\
(\mu g / L)\end{array}$ & $\begin{array}{l}\text { Lindane, } \\
\text { total } \\
(\mu g / L)\end{array}$ & $\begin{array}{c}\text { Toxa- } \\
\text { phene, } \\
\text { total } \\
(\mu g / L)\end{array}$ & $\begin{array}{c}\text { PCB, } \\
\text { total } \\
(\mu g / L)\end{array}$ & $\begin{array}{c}\text { Naph- } \\
\text { thalene, } \\
\text { polychlor } \\
\text { total } \\
(\mu g / L)\end{array}$ & $\begin{array}{c}\text { Meth- } \\
\text { oxychlor, } \\
\text { total } \\
(\mu g / L)\end{array}$ & $\begin{array}{c}\text { Mirex, } \\
\text { total } \\
\text { ( } \mu g / L)\end{array}$ \\
\hline $34 \mathrm{dcc} 2$ & $09-08-86$ & $<0.010$ & $<0.010$ & $<0.010$ & $<0.010$ & $<1.0$ & $<0.1$ & $<0.10$ & $<0.01$ & $<0.01$ \\
\hline 34aaal & $09-09-86$ & $<.010$ & $<.010$ & $<.010$ & $<.010$ & $<1.0$ & $<1$ & $<10$ & $<.01$ & $<.01$ \\
\hline $26 \mathrm{cdd}$ & $09-09-86$ & $<010$ & $<.010$ & $<.010$ & $<.010$ & $<1.0$ & $<.1$ & $<.10$ & $<.01$ & $<.01$ \\
\hline $27 \mathrm{cda}$ & $08-25-86$ & $<.010$ & $<.010$ & $<.010$ & $<.010$ & $<1.0$ & $<.1$ & $<.10$ & $<.01$ & $<.01$ \\
\hline 26dda 2 & $08-26-86$ & $<.010$ & $<.010$ & $<.010$ & $<.010$ & $<1.0$ & $<1$ & $<10$ & $<.01$ & $<.01$ \\
\hline $26 \mathrm{dac} 2$ & $08-26-86$ & $<.010$ & $<.010$ & $<.010$ & $<.010$ & $<1.0$ & $<1$ & $<.10$ & $<.01$ & $<.01$ \\
\hline 28 dad & $08-25-86$ & $<.010$ & $<.010$ & $<.010$ & $<.010$ & $<1.0$ & $<1$ & $<10$ & $<.01$ & $<.01$ \\
\hline $27 \mathrm{ada} 2$ & $08-27-86$ & $<.010$ & $<.010$ & $<.010$ & $<.010$ & $<1.0$ & $<1$ & $<.10$ & $<.01$ & $<.01$ \\
\hline $26 \mathrm{bbb}$ & $09-08-86$ & $<.010$ & $<.010$ & $<.010$ & $<.010$ & $<1.0$ & $<1$ & $<10$ & $<.01$ & $<.01$ \\
\hline
\end{tabular}


Table 9. Analytical results of ground-water samples, Rillito Creek basin Priority pollutants

\begin{tabular}{|c|c|c|c|c|c|c|c|c|c|c|}
\hline $\begin{array}{c}\text { Well } \\
\text { number } \\
\text { (D-13-14) }\end{array}$ & Date & $\begin{array}{c}\text { Para- } \\
\text { chloro- } \\
\text { meta } \\
\text { cresol, } \\
\text { total } \\
(\mu \mathrm{g} / \mathrm{L})\end{array}$ & $\begin{array}{c}\text { 2-Chloro- } \\
\text { phenol, } \\
\text { total } \\
(\mu g / L)\end{array}$ & $\begin{array}{c}\text { 2,4-Di- } \\
\text { chloro- } \\
\text { phenol, } \\
\text { total } \\
\text { ( } \mu \mathrm{g} / \mathrm{L})\end{array}$ & $\begin{array}{c}\text { 2,4,6-Tri- } \\
\text { chloro- } \\
\text { phenol, } \\
\text { total } \\
(\mu \mathrm{g} / \mathrm{L})\end{array}$ & $\begin{array}{c}\text { 2,4- } \\
\text { Dimethyl- } \\
\text { phenol, } \\
\text { total } \\
(\mu \mathrm{g} / \mathrm{L})\end{array}$ & $\begin{array}{c}\text { 4,6- } \\
\text { Dinitro- } \\
\text { ortho- } \\
\text { cresol, } \\
\text { total } \\
(\mu \mathrm{g} / \mathrm{L})\end{array}$ & $\begin{array}{c}\text { 2,4- } \\
\text { Dinitro- } \\
\text { phenol, } \\
\text { total } \\
(\mu \mathrm{g} / \mathrm{L})\end{array}$ & $\begin{array}{c}\text { 2-Nitro- } \\
\text { phenol, } \\
\text { total } \\
(\mu \mathrm{g} / \mathrm{L})\end{array}$ & $\begin{array}{c}\text { 4-Nitro- } \\
\text { phenol, } \\
\text { total } \\
(\mu \mathrm{g} / \mathrm{L})\end{array}$ \\
\hline \multirow[t]{3}{*}{$34 d c c 2$} & $09-08-86$ & $<5.0$ & $<6.0$ & $<6.0$ & $<5.0$ & $<6.0$ & $<30.0$ & $<20.0$ & $<6.0$ & $<30.0$ \\
\hline & $06-16-87$ & $<30.0$ & $<6.0$ & $<6.0$ & $<20.0$ & $<6.0$ & $<30.0$ & $<20.0$ & $<6.0$ & $<30.0$ \\
\hline & $03-27-89$ & $<30.0$ & 5.0 & $\delta .0$ & $<0.0$ & $<5.0$ & $<30.0$ & $<20.0$ & $\delta .0$ & $<30.0$ \\
\hline 34aaa1 & $09-09-86$ & $<5.0$ & $<6.0$ & $<6.0$ & $<5.0$ & $<6.0$ & $<30.0$ & $<20.0$ & $<6.0$ & $<30.0$ \\
\hline \multirow[t]{2}{*}{$27 \mathrm{ddc} 3$} & $08-27-86$ & $<5.0$ & $<6.0$ & $<6.0$ & $\delta .0$ & $<6.0$ & $<0.0$ & $<20.0$ & $<6.0$ & $<30.0$ \\
\hline & $06-15-87$ & $<30.0$ & $<6.0$ & $<6.0$ & $<20.0$ & $<6.0$ & $<30.0$ & $<20.0$ & $<6.0$ & $<30.0$ \\
\hline \multirow[t]{2}{*}{$26 \mathrm{cdd}$} & $09-09-86$ & 5.0 & $<6.0$ & $<6.0$ & $<5.0$ & $<6.0$ & $<30.0$ & $<20.0$ & $<6.0$ & $<30.0$ \\
\hline & $06-16-87$ & $<30.0$ & $<6.0$ & $<6.0$ & $<20.0$ & $<6.0$ & $<30.0$ & $<20.0$ & $<6.0$ & $<30.0$ \\
\hline $27 \mathrm{cda}$ & $08-25-86$ & $<5.0$ & $<6.0$ & $<6.0$ & $<5.0$ & $<6.0$ & $<30.0$ & $<20.0$ & $<6.0$ & $<30.0$ \\
\hline 26dda2 & $08-26-86$ & $<5.0$ & $<6.0$ & $<6.0$ & $<5.0$ & $<6.0$ & $<30.0$ & $<20.0$ & $<6.0$ & $<30.0$ \\
\hline \multirow[t]{3}{*}{$26 \mathrm{dac} 2$} & $08-26-86$ & $<5.0$ & $<6.0$ & $<6.0$ & $<5.0$ & $<6.0$ & $<30.0$ & $<20.0$ & $<6.0$ & $<30.0$ \\
\hline & $06-16-87$ & $<30.0$ & $<6.0$ & $<6.0$ & $<20.0$ & $<6.0$ & $<30.0$ & $<20.0$ & $<6.0$ & $<30.0$ \\
\hline & $03-29-89$ & $<30.0$ & $\delta .0$ & -5.0 & $<20.0$ & $<5.0$ & $<30.0$ & $<20.0$ & 5.0 & $<30.0$ \\
\hline $28 \mathrm{dad}$ & $08-25-86$ & $<5.0$ & $<6.0$ & $<6.0$ & $<5.0$ & $<6.0$ & $<30.0$ & $<20.0$ & $<6.0$ & $<30.0$ \\
\hline \multirow[t]{2}{*}{ 27ada2 } & $08-27-86$ & $<5.0$ & $<6.0$ & $<6.0$ & $<5.0$ & $<6.0$ & $<30.0$ & $<20.0$ & $<6.0$ & $<30.0$ \\
\hline & $06-15-87$ & $<30.0$ & $<6.0$ & $<6.0$ & $<0.0$ & $<6.0$ & $<30.0$ & $<20.0$ & $<6.0$ & $<30.0$ \\
\hline \multirow[t]{3}{*}{$27 \mathrm{bdb} 4$} & $09-08-86$ & $<5.0$ & $<6.0$ & $<6.0$ & $<5.0$ & $<6.0$ & $<30.0$ & $<20.0$ & $<6.0$ & $<30.0$ \\
\hline & $06-15-87$ & $<0.0$ & $<6.0$ & $<6.0$ & $<20.0$ & $<6.0$ & $<30.0$ & $<20.0$ & $<6.0$ & $<30.0$ \\
\hline & $03-27-89$ & $<30.0$ & 5.0 & $\delta .0$ & $<0.0$ & 5.0 & $<30.0$ & $<20.0$ & $\$ .0$ & $<30.0$ \\
\hline $26 \mathrm{bbb}$ & $09-08-86$ & $<10.0$ & $<12.0$ & $<12.0$ & $<10.0$ & $<12.0$ & $<60.0$ & $<40.0$ & $<12.0$ & $<60.0$ \\
\hline
\end{tabular}

\begin{tabular}{|c|c|c|c|c|c|c|c|c|c|c|}
\hline $\begin{array}{c}\text { Well } \\
\text { number } \\
(D-13-14)\end{array}$ & Date & $\begin{array}{c}\text { Penta- } \\
\text { chloro- } \\
\text { phenol, } \\
\text { total } \\
(\mu \mathrm{g} / \mathrm{L})\end{array}$ & $\begin{array}{c}\text { Phenol, } \\
\text { (C6h-50h) } \\
\text { total } \\
(\mu \mathrm{g} / \mathrm{L})\end{array}$ & $\begin{array}{c}\text { Ace- } \\
\text { naph- } \\
\text { thene, } \\
\text { total } \\
(\mu \mathrm{g} / \mathrm{L})\end{array}$ & $\begin{array}{c}\text { Ace- } \\
\text { naph- } \\
\text { thylene, } \\
\text { total } \\
(\mu \mathrm{g} / \mathrm{L})\end{array}$ & $\begin{array}{c}\text { Anthra- } \\
\text { cene, } \\
\text { total } \\
(\mu g / L)\end{array}$ & $\begin{array}{c}\text { Benzo A } \\
\text { anthra- } \\
\text { cene, 1,2- } \\
\text { benzan- } \\
\text { thracene, } \\
\text { total } \\
(\mu \mathrm{g} / \mathrm{L})\end{array}$ & $\begin{array}{c}\text { Benzo B } \\
\text { fluor- } \\
\text { anthene, } \\
\text { total } \\
(\mu g / L)\end{array}$ & $\begin{array}{c}\text { Benzo K } \\
\text { fluor- } \\
\text { anthene, } \\
\text { total } \\
(\mu \mathrm{g} / \mathrm{L})\end{array}$ & $\begin{array}{c}\text { Benzo } \\
\text { A } \\
\text { pyrene, } \\
\text { total } \\
(\mu g / L)\end{array}$ \\
\hline \multirow[t]{3}{*}{$34 \mathrm{dcc} 2$} & $09-08-86$ & $<30.0$ & $<6.0$ & 5.0 & 5.0 & $<5.0$ & $<10.0$ & $<10.0$ & $<10.0$ & $<10.0$ \\
\hline & $06-16-87$ & $<30.0$ & 39.0 & $\delta .0$ & 5.0 & $<5.0$ & $<10.0$ & $<10.0$ & $<10.0$ & $<10.0$ \\
\hline & $03-27-89$ & $<30.0$ & 16.0 & $\delta .0$ & $\delta .0$ & $\delta .0$ & 5.0 & $<10.0$ & $<10.0$ & $<10.0$ \\
\hline 34aaal & $09-09-86$ & $<30.0$ & $<6.0$ & $\delta .0$ & $\delta .0$ & $<5.0$ & $<10.0$ & $<10.0$ & $<10.0$ & $<10.0$ \\
\hline \multirow[t]{2}{*}{$27 \mathrm{ddc} 3$} & $08-27-86$ & $<30.0$ & $<6.0$ & $\delta .0$ & $<.0$ & $<5.0$ & $<10.0$ & $<10.0$ & $<10.0$ & $<10.0$ \\
\hline & $06-15-87$ & $<30.0$ & $<5.0$ & $<.0$ & $<5.0$ & $<5.0$ & $<10.0$ & $<10.0$ & $<10.0$ & $<10.0$ \\
\hline \multirow[t]{2}{*}{$26 \mathrm{cdd}$} & $09-09-86$ & $<30.0$ & $<6.0$ & $\delta .0$ & 5.0 & $\delta .0$ & $<10.0$ & $<10.0$ & $<10.0$ & $<10.0$ \\
\hline & $06-16-87$ & $<30.0$ & $<5.0$ & 5.0 & $\delta .0$ & 5.0 & $<10.0$ & $<10.0$ & $<10.0$ & $<10.0$ \\
\hline $27 \mathrm{cda}$ & $08-25-86$ & $<30.0$ & $<6.0$ & 5.0 & $<5.0$ & $<.0$ & $<10.0$ & $<10.0$ & $<10.0$ & $<10.0$ \\
\hline 26dda2 & $08-26-86$ & $<30.0$ & $<6.0$ & -5.0 & $\infty .0$ & $<5.0$ & $<10.0$ & $<10.0$ & $<10.0$ & $<10.0$ \\
\hline \multirow[t]{3}{*}{$26 \mathrm{dac} 2$} & $08-26-86$ & $<30.0$ & $<6.0$ & $<.0$ & $\delta .0$ & $\delta .0$ & $<10.0$ & $<10.0$ & $<10.0$ & $<10.0$ \\
\hline & $06-16-87$ & $<30.0$ & $<5.0$ & 5.0 & 5.0 & $<5.0$ & $<10.0$ & $<10.0$ & $<10.0$ & $<10.0$ \\
\hline & $03-29-89$ & $<30.0$ & $<5.0$ & $\delta .0$ & $\infty .0$ & 5.0 & $\delta .0$ & $<10.0$ & $<10.0$ & $<10.0$ \\
\hline $28 \mathrm{dad}$ & $08-25-86$ & $<0.0$ & $<6.0$ & $\delta .0$ & $<5.0$ & $<5.0$ & $<10.0$ & $<10.0$ & $<10.0$ & $<10.0$ \\
\hline \multirow[t]{2}{*}{ 27ada2 } & $08-27-86$ & $<30.0$ & $<6.0$ & $\delta .0$ & $<.0$ & $<5.0$ & $<10.0$ & $<10.0$ & $<10.0$ & $<10.0$ \\
\hline & $06-15-87$ & $<30.0$ & $<5.0$ & $\delta .0$ & $<5.0$ & 5.0 & $<10.0$ & $<10.0$ & $<10.0$ & $<10.0$ \\
\hline \multirow[t]{3}{*}{$27 \mathrm{bdb} 4$} & $09-08-86$ & $<30.0$ & $<6.0$ & $\delta .0$ & 5.0 & $\delta .0$ & $<10.0$ & $<10.0$ & $<10.0$ & $<10.0$ \\
\hline & $06-15-87$ & $<30.0$ & $<5.0$ & $<.0$ & 5.0 & $<5.0$ & $<10.0$ & $<10.0$ & $<10.0$ & $<10.0$ \\
\hline & $03-27-89$ & $<30.0$ & 5.0 & 5.0 & $\delta .0$ & $<5.0$ & $\delta .0$ & $<10.0$ & $<10.0$ & $<10.0$ \\
\hline $26 \mathrm{bbb}$ & $09-08-86$ & $<60.0$ & $<12.0$ & $<10.0$ & $<10.0$ & $<10.0$ & $<20.0$ & $<20.0$ & $<20.0$ & $<20.0$ \\
\hline
\end{tabular}


Table 9. Analytical results of ground-water samples, Rillito Creek basin-Continued Priority pollutants-Continued

\begin{tabular}{|c|c|c|c|c|c|c|c|c|c|c|}
\hline $\begin{array}{c}\text { Well } \\
\text { number } \\
(D-13-14)\end{array}$ & Date & $\begin{array}{c}\text { Benzogh I } \\
\text { perylene, } \\
\text { 1, 12- } \\
\text { benzo- } \\
\text { perylene, } \\
\text { total } \\
(\mu g / L)\end{array}$ & $\begin{array}{c}\text { N-Butyl- } \\
\text { benzly- } \\
\text { phthalate, } \\
\text { total } \\
(\mu \mathrm{g} / \mathrm{L})\end{array}$ & $\begin{array}{c}\text { Bis (2- } \\
\text { chloro- } \\
\text { ethoxy) } \\
\text { methane, } \\
\text { total } \\
(\mu g / L)\end{array}$ & $\begin{array}{l}\text { Bis (2- } \\
\text { chloro- } \\
\text { ethyl) } \\
\text { ether, is } \\
\text { total eth } \\
(\mu g / L)\end{array}$ & $\begin{array}{l}\text { Bis (2 } \\
\text { chloro- } \\
\text { isopropyl) } \\
\text { ather, total } \\
(\mu \mathrm{g} / \mathrm{L})\end{array}$ & $\begin{array}{l}\text { 4-Bromo- } \\
\text { phenyl } \\
\text { phenyl } \\
\text { ether, } \\
\text { total } \\
(\mu \mathrm{g} / \mathrm{L})\end{array}$ & $\begin{array}{c}\text { 2-Chloro- } \\
\text { naphtha- } \\
\text { lene, } \\
\text { total } \\
(\mu g / L)\end{array}$ & $\begin{array}{l}\text { 4-Chloro- } \\
\text { phenyl } \\
\text { phenyl } \\
\text { ether, } \\
\text { total } \\
(\mu \mathrm{g} / \mathrm{L})\end{array}$ & $\begin{array}{c}\text { Chrysene, } \\
\text { total } \\
(\mu \mathrm{g} / \mathrm{L})\end{array}$ \\
\hline \multirow[t]{3}{*}{$34 \mathrm{dcc} 2$} & 09-08-86 & $<10.0$ & $<5.0$ & $<5.0$ & $<5.0$ & $<5.0$ & 5.0 & $<5.0$ & $<5.0$ & $<10.0$ \\
\hline & $06-16-87$ & $<10.0$ & $<5.0$ & $<5.0$ & $<5.0$ & $<5.0$ & 5.0 & $<5.0$ & $<5.0$ & $<10.0$ \\
\hline & $03-27-89$ & $<10.0$ & $<5.0$ & $<5.0$ & $<5.0$ & $<5.0$ & 5.0 & $<5.0$ & $<5.0$ & $<10.0$ \\
\hline 34aaal & 09-09-86 & $<10.0$ & $<5.0$ & $<5.0$ & $<5.0$ & $<5.0$ & $<.0$ & $<5.0$ & $<5.0$ & $<10.0$ \\
\hline \multirow[t]{2}{*}{$27 \mathrm{ddc} 3$} & $08-27-86$ & $<10.0$ & $<5.0$ & $<5.0$ & $<5.0$ & $<5.0$ & 5.0 & $<5.0$ & $<5.0$ & $<10.0$ \\
\hline & $06-15-87$ & $<10.0$ & $<5.0$ & $<5.0$ & $<5.0$ & $<5.0$ & 5.0 & $<5.0$ & $<5.0$ & $<10.0$ \\
\hline \multirow[t]{2}{*}{$26 \mathrm{cdd}$} & $09-09-86$ & $<10.0$ & $<5.0$ & $<5.0$ & $<5.0$ & $<5.0$ & 5.0 & $<5.0$ & $<5.0$ & $<10.0$ \\
\hline & $06-16-87$ & $<10.0$ & $<5.0$ & $<5.0$ & $<5.0$ & $<5.0$ & $\delta .0$ & $<5.0$ & $<5.0$ & $<10.0$ \\
\hline $27 \mathrm{cda}$ & $08-25-86$ & $<10.0$ & $<5.0$ & $<5.0$ & $<5.0$ & $<5.0$ & 5.0 & $<5.0$ & $<5.0$ & $<10.0$ \\
\hline $26 \mathrm{dda} 2$ & $08-26-86$ & $<10.0$ & $<5.0$ & $<5.0$ & $<5.0$ & $<5.0$ & $\delta .0$ & $<5.0$ & $<5.0$ & $<10.0$ \\
\hline \multirow[t]{3}{*}{$26 \mathrm{dac} 2$} & $08-26-86$ & $<10.0$ & $<5.0$ & $<5.0$ & $<5.0$ & $<5.0$ & 5.0 & $<5.0$ & $<5.0$ & $<10.0$ \\
\hline & $06-16-87$ & $<10.0$ & $<5.0$ & $<5.0$ & $<5.0$ & $<5.0$ & 5.0 & $<5.0$ & $<5.0$ & $<10.0$ \\
\hline & 03-29-89 & $<10.0$ & 5.0 & $<.0$ & $<5.0$ & $<5.0$ & 5.0 & $<5.0$ & $<5.0$ & $<10.0$ \\
\hline 28dad & $08-25-86$ & $<10.0$ & $<5.0$ & $<5.0$ & $<5.0$ & $<5.0$ & $\delta .0$ & $<5.0$ & $<5.0$ & $<10.0$ \\
\hline \multirow[t]{2}{*}{$27 \mathrm{ad} 22$} & $08-27-86$ & $<10.0$ & $<5.0$ & $<5.0$ & $<5.0$ & $<5.0$ & $\delta .0$ & $<5.0$ & $<5.0$ & $<10.0$ \\
\hline & $06-15-87$ & $<10.0$ & $<5.0$ & $<5.0$ & $<5.0$ & $<5.0$ & $\delta .0$ & $<5.0$ & $<5.0$ & $<10.0$ \\
\hline \multirow[t]{3}{*}{$27 \mathrm{bdb} 4$} & $09-08-86$ & $<10.0$ & $<5.0$ & $<5.0$ & $<5.0$ & $<5.0$ & 5.0 & $<5.0$ & $<5.0$ & $<10.0$ \\
\hline & $06-15-87$ & $<10.0$ & $<5.0$ & $<5.0$ & $<5.0$ & $<5.0$ & 5.0 & $<5.0$ & $<5.0$ & $<10.0$ \\
\hline & 03-27-89 & $<10.0$ & $<5.0$ & $<5.0$ & $<5.0$ & $<5.0$ & $\delta .0$ & $<5.0$ & $<5.0$ & $<10.0$ \\
\hline $26 \mathrm{bbb}$ & 09-08-86 & $<20.0$ & $<10.0$ & $<10.0$ & $<10.0$ & $<10.0$ & $<10.0$ & $<10.0$ & $<10.0$ & $<20.0$ \\
\hline $\begin{array}{c}\text { Well } \\
\text { number } \\
(D-13-14)\end{array}$ & Date & $\begin{array}{c}1,2,5,6 \\
\text { Dibenz- } \\
\text { anthra- } \\
\text { cene, } \\
\text { total } \\
(\mu g / L)\end{array}$ & $\begin{array}{c}\text { Di-n- } \\
\text { butyl- } \\
\text { phthalate, } \\
\text { total } \\
(\mu g / L)\end{array}$ & $\begin{array}{c}1,2- \\
\text { Dichloro- } \\
\text { benzene, } \\
\text { total } \\
(\mu g / L)\end{array}$ & $\begin{array}{c}1,3- \\
\text { Dichloro- } \\
\text { benzene, } \\
\text { total } \\
(\mu g / L)\end{array}$ & $\begin{array}{c}1,4- \\
\text { o- } \\
\text { eichloro- } \\
\text { e, benzene, } \\
\text { total } \\
(\mu g / L)\end{array}$ & $\begin{array}{c}\text { Diethyl- } \\
\text { phthal- } \\
\text { ate, } \\
\text { total } \\
(\mu \mathrm{g} / \mathrm{L})\end{array}$ & $\begin{array}{c}\text { Di- } \\
\text { methyl- } \\
\text { phthal- } \\
\text { ate, } \\
\text { total } \\
(\mu g / L)\end{array}$ & $\begin{array}{c}\text { 2,4- } \\
\text { Dinitro- } \\
\text { toluene, } \\
\text { total } \\
(\mu g / L)\end{array}$ & $\begin{array}{c}\text { 2,6- } \\
\text { Dinitro- } \\
\text { toluene, } \\
\text { total } \\
(\mu g / L)\end{array}$ \\
\hline \multirow[t]{3}{*}{$34 \mathrm{dcc} 2$} & $09-08-86$ & $<10.0$ & 5.0 & $<3.0$ & $<3.0$ & $<.0$ & $<5.0$ & $<5.0$ & 5.0 & 5.0 \\
\hline & $06-16-87$ & $<10.0$ & $\delta .0$ & $\delta .0$ & $<.0$ & $<5.0$ & $<5.0$ & $<5.0$ & $\delta .0$ & 5.0 \\
\hline & $03-27-89$ & $<10.0$ & $\delta .0$ & 5.0 & $<5.0$ & $<5.0$ & $<5.0$ & $<5.0$ & 5.0 & 5.0 \\
\hline 34aaal & $09-09-86$ & $<10.0$ & $\delta .0$ & $\delta .0$ & $<5.0$ & $<5.0$ & $<5.0$ & $<5.0$ & $<5.0$ & $\delta .0$ \\
\hline \multirow[t]{2}{*}{$27 \mathrm{ddc} 3$} & $08-27-86$ & $<10.0$ & $\delta .0$ & $<3.0$ & $<3.0$ & $<3.0$ & 5.0 & 5.0 & $<5.0$ & $\delta .0$ \\
\hline & $06-15-87$ & $<10.0$ & $\delta .0$ & 5.0 & $<5.0$ & $\delta .0$ & $<5.0$ & $<5.0$ & 5.0 & 5.0 \\
\hline \multirow[t]{2}{*}{$26 \mathrm{cdd}$} & $09-09-86$ & $<10.0$ & $\delta .0$ & $<3.0$ & $<3.0$ & $<3.0$ & $<5.0$ & $<5.0$ & $\delta .0$ & 5.0 \\
\hline & $06-16-87$ & $<10.0$ & $<.0$ & $\delta .0$ & $<5.0$ & 5.0 & $<5.0$ & $<5.0$ & $<5.0$ & $\delta .0$ \\
\hline $27 \mathrm{cda}$ & $08-25-86$ & $<10.0$ & $\delta .0$ & $<3.0$ & $<3.0$ & $<3.0$ & $<5.0$ & 5.0 & 5.0 & $<5.0$ \\
\hline $26 \mathrm{dda} 2$ & $08-26-86$ & $<10.0$ & $\delta .0$ & $<3.0$ & $<3.0$ & $<3.0$ & 5.0 & $<.0$ & 5.0 & 5.0 \\
\hline \multirow[t]{3}{*}{ 26dac2 } & $08-26-86$ & $<10.0$ & $\delta .0$ & $<3.0$ & $<3.0$ & $<.0$ & 5.0 & $<5.0$ & $\delta .0$ & 5.0 \\
\hline & $06-16-87$ & $<10.0$ & $\delta .0$ & 5.0 & $<5.0$ & $\delta .0$ & $<5.0$ & $<5.0$ & 5.0 & $\delta .0$ \\
\hline & 03-29-89 & $<10.0$ & 5.0 & 5.0 & $<5.0$ & 5.0 & 5.0 & $<5.0$ & $\delta .0$ & 5.0 \\
\hline 28 dad & $08-25-86$ & $<10.0$ & 5.0 & $<3.0$ & $<3.0$ & $<3.0$ & $<5.0$ & $<5.0$ & 5.0 & 5.0 \\
\hline \multirow[t]{2}{*}{ 27ada2 } & $08-27-86$ & $<10.0$ & 5.0 & $<3.0$ & $<3.0$ & $<.0$ & $\delta .0$ & $<5.0$ & $\delta .0$ & $\delta .0$ \\
\hline & $06-15-87$ & $<10.0$ & $\delta .0$ & $\delta .0$ & $<5.0$ & $\delta .0$ & $<5.0$ & $<5.0$ & 5.0 & 5.0 \\
\hline \multirow[t]{3}{*}{$27 \mathrm{bdb} 4$} & $09-08-86$ & $<10.0$ & -5.0 & $<.0$ & $<3.0$ & $<3.0$ & $<5.0$ & $<5.0$ & 5.0 & 5.0 \\
\hline & $06-15-87$ & $<10.0$ & $\delta .0$ & 5.0 & $<5.0$ & 5.0 & $<5.0$ & $<5.0$ & 5.0 & $\delta .0$ \\
\hline & $03-27-89$ & $<10.0$ & $\delta .0$ & 5.0 & $<5.0$ & $\delta .0$ & 5.0 & $<5.0$ & 5.0 & 5.0 \\
\hline $26 \mathrm{bbb}$ & 09-08-86 & $<0.0$ & $<10.0$ & $<3.0$ & $<3.0$ & $<3.0$ & $<10.0$ & $<10.0$ & $<10.0$ & $<10.0$ \\
\hline
\end{tabular}


Table 9. Analytical results of ground-water samples, Rillito Creek basin-Continued Priority pollutants-Continued

\begin{tabular}{|c|c|c|c|c|c|c|c|c|c|c|}
\hline $\begin{array}{c}\text { Well } \\
\text { number } \\
(D-13-14)\end{array}$ & Date & $\begin{array}{c}\text { Di-n- } \\
\text { octyl- } \\
\text { phthal- } \\
\text { ate, } \\
\text { total } \\
(\mu g / L)\end{array}$ & $\begin{array}{c}\text { Bis (2- } \\
\text { ethyl- } \\
\text { hexyl) } \\
\text { phthal- } \\
\text { ate, } \\
\text { total } \\
(\mu \mathrm{g} / \mathrm{L})\end{array}$ & $\begin{array}{c}\text { Fluorene, } \\
\text { total } \\
(\mu g / L)\end{array}$ & $\begin{array}{c}\text { Fluor- } \\
\text { anthene, } \\
\text { total } \\
(\mu \mathrm{g} / \mathrm{L})\end{array}$ & $\begin{array}{c}\text { Hexa- } \\
\text { chloro- } \\
\text { benzene, } \\
\text { total } \\
(\mu g / L)\end{array}$ & $\begin{array}{c}\text { Hexa- } \\
\text { chloro- } \\
\text { butadlene, } \\
\text { total } \\
(\mu \mathrm{g} / \mathrm{L})\end{array}$ & $\begin{array}{l}\text { Hexa- } \\
\text { chloro- } \\
\text { cyclopent- } \\
\text { adiene, } \\
\text { total } \\
(\mu \mathrm{g} / \mathrm{L})\end{array}$ & $\begin{array}{l}\text { Hexa- } \\
\text { chloro- } \\
\text { ethane, } \\
\text { total } \\
(\mu \mathrm{g} / \mathrm{L})\end{array}$ & $\begin{array}{c}\text { Indeno } \\
(1,2,3-c d) \\
\text { pyrene, } \\
\text { total } \\
(\mu g / L)\end{array}$ \\
\hline \multirow[t]{3}{*}{ 34dcc2 } & $09-08-86$ & $<10.0$ & $<5.0$ & $<5.0$ & 5.0 & $<.0$ & 5.0 & 5.0 & 5.0 & $<10.0$ \\
\hline & $06-16-87$ & $<10.0$ & 5.0 & $<5.0$ & 5.0 & 5.0 & $\delta .0$ & $<5.0$ & 5.0 & $<10.0$ \\
\hline & $03-27-89$ & $<10.0$ & $\delta .0$ & 5.0 & 5.0 & 5.0 & $<5.0$ & $<5.0$ & 5.0 & $<10.0$ \\
\hline 34aaal & $09-09-86$ & $<10.0$ & $<5.0$ & $<.0$ & 5.0 & $<5.0$ & $<.0$ & 5.0 & $\delta .0$ & $<10.0$ \\
\hline \multirow[t]{2}{*}{$27 \mathrm{ddc} 3$} & $08-27-86$ & $<10.0$ & $<.0$ & $<5.0$ & 5.0 & 5.0 & $<.0$ & 5.0 & 5.0 & $<10.0$ \\
\hline & $06-15-87$ & $<10.0$ & $<5.0$ & $<5.0$ & 5.0 & $\delta .0$ & $<5.0$ & $<5.0$ & $\delta .0$ & $<10.0$ \\
\hline \multirow[t]{2}{*}{$26 \mathrm{cdd}$} & $09-09-86$ & $<10.0$ & $<5.0$ & $<5.0$ & 5.0 & 5.0 & 5.0 & $\delta .0$ & 5.0 & $<10.0$ \\
\hline & $06-16-87$ & 17.0 & $<5.0$ & $<5.0$ & 5.0 & 5.0 & 5.0 & $<.0$ & $<.0$ & $<10.0$ \\
\hline $27 \mathrm{cda}$ & $08-25-86$ & $<10.0$ & 5.0 & $<5.0$ & 5.0 & 5.0 & 5.0 & $<.0$ & 5.0 & $<10.0$ \\
\hline $26 \mathrm{dda} 2$ & $08-26-86$ & $<10.0$ & 5.0 & 5.0 & $<5.0$ & 5.0 & 5.0 & 5.0 & 5.0 & $<10.0$ \\
\hline \multirow[t]{3}{*}{ 26dac2 } & $08-26-86$ & $<10.0$ & $<5.0$ & $<5.0$ & $\delta .0$ & 5.0 & 5.0 & $<.0$ & $<5.0$ & $<10.0$ \\
\hline & $06-16-87$ & $<10.0$ & $\delta .0$ & $<5.0$ & $<5.0$ & $<.0$ & 5.0 & $<5.0$ & 5.0 & $<10.0$ \\
\hline & $03-29-89$ & $<10.0$ & 5.0 & 5.0 & $<5.0$ & 5.0 & 5.0 & $<5.0$ & $\infty .0$ & $<10.0$ \\
\hline 28 dad & $08-25-86$ & $<10.0$ & $<.0$ & 5.0 & $\delta .0$ & 5.0 & 5.0 & $\delta .0$ & $\delta .0$ & $<10.0$ \\
\hline \multirow[t]{2}{*}{ 27ada2 } & $08-27-86$ & $<10.0$ & 5.0 & 5.0 & $<.0$ & 5.0 & 5.0 & 5.0 & 5.0 & $<10.0$ \\
\hline & $06-15-87$ & $<10.0$ & $<.0$ & 5.0 & $<5.0$ & 5.0 & 5.0 & 5.0 & 5.0 & $<10.0$ \\
\hline \multirow[t]{3}{*}{$27 \mathrm{bdb} 4$} & $09-08-86$ & $<10.0$ & $<5.0$ & $<5.0$ & $<5.0$ & 5.0 & 5.0 & $<5.0$ & $<.0$ & $<10.0$ \\
\hline & $06-15-87$ & $<10.0$ & $<.0$ & 5.0 & 5.0 & 5.0 & $\delta .0$ & $\delta .0$ & $<.0$ & $<10.0$ \\
\hline & $03-27-89$ & $<10.0$ & $<5.0$ & $\delta .0$ & 5.0 & 5.0 & 5.0 & $\delta .0$ & $<.0$ & $<10.0$ \\
\hline $26 \mathrm{bbb}$ & $09-08-86$ & $<20.0$ & $<10.0$ & $<10.0$ & $<10.0$ & $<10.0$ & $<10.0$ & $<10.0$ & $<10.0$ & $<0.0$ \\
\hline
\end{tabular}

\begin{tabular}{|c|c|c|c|c|c|c|c|c|c|c|}
\hline $\begin{array}{c}\text { Well } \\
\text { number } \\
\text { (D-13-14) }\end{array}$ & Date & $\begin{array}{c}\text { Isopho- } \\
\text { rone, } \\
\text { total } \\
(\mu \mathrm{g} / \mathrm{L})\end{array}$ & $\begin{array}{c}\text { Naphtha- } \\
\text { lene, } \\
\text { total } \\
(\mu g / L)\end{array}$ & $\begin{array}{c}\text { Nitro- } \\
\text { benzene, } \\
\text { total } \\
(\mu \mathrm{g} / \mathrm{L})\end{array}$ & $\begin{array}{c}\text { N-nitro- } \\
\text { sodi- } \\
\text { mothyl- } \\
\text { amine, } \\
\text { total } \\
(\mu \mathrm{g} / \mathrm{L})\end{array}$ & $\begin{array}{c}\text { N-nitro- } \\
\text { sodi- } \\
\text { phenyi- } \\
\text { amine, } \\
\text { total } \\
(\mu \mathrm{g} / \mathrm{L})\end{array}$ & $\begin{array}{l}\text { N-nitro- } \\
\text { sodi-n- } \\
\text { propyl- } \\
\text { amine, } \\
\text { total } \\
(\mu g / L)\end{array}$ & $\begin{array}{c}\text { Phenan- } \\
\text { threne, } \\
\text { total } \\
(\mu \mathrm{g} / \mathrm{L})\end{array}$ & $\begin{array}{c}\text { Pyrene, } \\
\text { lotal } \\
(\mu \mathrm{g} / \mathrm{L})\end{array}$ & $\begin{array}{c}\text { 1,2,4- } \\
\text { Trichloro- } \\
\text { benzene, } \\
\text { total } \\
(\mu \mathrm{g} / \mathrm{L})\end{array}$ \\
\hline \multirow[t]{3}{*}{$34 d c c 2$} & $09-08-86$ & 5.0 & $<.0$ & 5.0 & 5.0 & 5.0 & 5.0 & 5.0 & $\delta .0$ & 5.0 \\
\hline & $06-16-87$ & $<5.0$ & $<.0$ & -5.0 & $<.0$ & $<.0$ & $<.0$ & 5.0 & $<.0$ & $<.0$ \\
\hline & $03-27-89$ & $<5.0$ & 5.0 & $\delta .0$ & $<.0$ & $<.0$ & $<.0$ & $<5.0$ & $<.0$ & 5.0 \\
\hline 34aaa 1 & $09-09-86$ & $<5.0$ & 5.0 & $<.0$ & $<.0$ & $<.0$ & $<.0$ & 5.0 & 8.0 & $<.0$ \\
\hline \multirow[t]{2}{*}{$27 \mathrm{ddc} 3$} & $08-27-86$ & $\delta .0$ & 5.0 & $<.0$ & 5.0 & $<5.0$ & $<.0$ & $<.0$ & $<.0$ & 5.0 \\
\hline & $06-15-87$ & $<.0$ & 5.0 & $<.0$ & $\delta .0$ & $<5.0$ & $<.0$ & $<.0$ & $<5.0$ & $\delta .0$ \\
\hline \multirow[t]{2}{*}{$26 \mathrm{cdd}$} & 09-09-86 & $\delta .0$ & 5.0 & 5.0 & $<.0$ & $<5.0$ & $<5.0$ & $<.0$ & $<.0$ & $<.0$ \\
\hline & $06-16-87$ & $<5.0$ & -5.0 & $<5.0$ & $<.0$ & $<5.0$ & $<.0$ & $<.0$ & $<.0$ & $<.0$ \\
\hline $27 \mathrm{cda}$ & $08-25-86$ & $<.0$ & 5.0 & -5.0 & -5.0 & $<.0$ & 5.0 & 5.0 & $\delta .0$ & 5.0 \\
\hline $26 \mathrm{dda} 2$ & $08-26-86$ & $<5.0$ & -5.0 & 5.0 & $<.0$ & 5.0 & $<.0$ & -5.0 & 5.0 & $\delta .0$ \\
\hline \multirow[t]{3}{*}{$26 \mathrm{dac} 2$} & $08-26-86$ & $<5.0$ & -5.0 & -5.0 & 5.0 & $<5.0$ & -5.0 & -5.0 & 5.0 & 5.0 \\
\hline & $06-16-87$ & $<5.0$ & 5.0 & $<5.0$ & $<.0$ & $<5.0$ & $\delta .0$ & -5.0 & $<.0$ & $<.0$ \\
\hline & 03-29-89 & 5.0 & 5.0 & -5.0 & -5.0 & $<.0$ & $<.0$ & -5.0 & $\delta .0$ & -5.0 \\
\hline $28 \mathrm{dad}$ & 08-25-86 & 5.0 & 5.0 & $<.0$ & $\delta .0$ & 5.0 & $<.0$ & $<.0$ & $\delta .0$ & $\delta .0$ \\
\hline \multirow[t]{2}{*}{ 27ada2 } & 08-27-86 & $<.0$ & 5.0 & $<.0$ & -5.0 & $<5.0$ & $<5.0$ & $<.0$ & $<.0$ & $<.0$ \\
\hline & $06-15-87$ & $<5.0$ & -5.0 & -5.0 & $<.0$ & $<.0$ & $<.0$ & -5.0 & 5.0 & 5.0 \\
\hline \multirow[t]{3}{*}{$27 \mathrm{bdb} 4$} & 09-08-86 & $<5.0$ & 5.0 & 5.0 & $\delta .0$ & 5.0 & $\delta .0$ & -5.0 & 8.0 & -5.0 \\
\hline & $06-15-87$ & $<5.0$ & 5.0 & $\delta .0$ & $<.0$ & $<5.0$ & $<.0$ & 5.0 & $<.0$ & $<.0$ \\
\hline & $03-27-89$ & $<5.0$ & $\delta .0$ & $\delta .0$ & $<.0$ & 5.0 & $<5.0$ & -5.0 & 5.0 & $<.0$ \\
\hline $26 \mathrm{bbb}$ & 09-08-86 & $<10.0$ & $<10.0$ & $<10.0$ & $<10.0$ & $<10.0$ & $<10.0$ & $<10.0$ & $<10.0$ & $<10.0$ \\
\hline
\end{tabular}


Table 9. Analytical results of ground-water samples, Rillito Creek basin-Continued Volatile organic compounds

\begin{tabular}{|c|c|c|c|c|c|c|c|c|c|}
\hline $\begin{array}{c}\text { Well } \\
\text { number } \\
\text { (D-13-14) }\end{array}$ & Date & $\begin{array}{c}\text { Benzene, } \\
\text { totai } \\
(\mu \mathrm{g} / \mathrm{L})\end{array}$ & $\begin{array}{c}\text { Bromo- } \\
\text { form, } \\
\text { total } \\
(\mu \mathrm{g} / \mathrm{L})\end{array}$ & $\begin{array}{l}\text { Carbon- } \\
\text { tetrachlo- } \\
\text { ride, } \\
\text { total } \\
(\mu g / L)\end{array}$ & $\begin{array}{c}\text { Chloro- } \\
\text { benzene, } \\
\text { total } \\
(\mu \mathrm{g} / \mathrm{L})\end{array}$ & $\begin{array}{c}\text { Chloro- } \\
\text { dlbromo- } \\
\text { methane, } \\
\text { total } \\
(\mu g / L)\end{array}$ & $\begin{array}{c}\text { Chloro- } \\
\text { ethane, } \\
\text { total } \\
(\mu g / L)\end{array}$ & $\begin{array}{l}\text { 2-Chloro- } \\
\text { ethylvinyl- } \\
\text { ether, } \\
\text { total } \\
(\mu \mathrm{g} / \mathrm{L})\end{array}$ & $\begin{array}{l}\text { Chloro- } \\
\text { form, } \\
\text { total } \\
(\mu \mathrm{g} / \mathrm{L})\end{array}$ \\
\hline \multirow[t]{3}{*}{$34 \mathrm{dcc} 2$} & $09-08-86$ & $<3.0$ & $<3.0$ & $<3.0$ & $<3.0$ & $<3.0$ & $<3.0$ & $<3.0$ & $<3.0$ \\
\hline & $06-16-87$ & $<3.0$ & $<3.0$ & $<3.0$ & $<3.0$ & $<3.0$ & $<3.0$ & $<3.0$ & $<3.0$ \\
\hline & 03-27-89 & $<3.0$ & $<3.0$ & $<3.0$ & $<3.0$ & $<3.0$ & $<3.0$ & $<3.0$ & $<3.0$ \\
\hline 34aaal & $03-29-89$ & $<3.0$ & $<3.0$ & $<3.0$ & $<3.0$ & $<3.0$ & $<3.0$ & $<3.0$ & $<3.0$ \\
\hline \multirow[t]{2}{*}{$27 \mathrm{ddc} 3$} & $08-27-86$ & $<3.0$ & $<3.0$ & $<3.0$ & $<3.0$ & $<3.0$ & $<3.0$ & $<3.0$ & $<3.0$ \\
\hline & 03-28-89 & $<3.0$ & $<3.0$ & $<3.0$ & $<3.0$ & $<3.0$ & $<3.0$ & $<3.0$ & $<3.0$ \\
\hline $26 \mathrm{cdd}$ & 09-09-86 & $<3.0$ & $<3.0$ & $<3.0$ & $<3.0$ & $<3.0$ & $<3.0$ & $<3.0$ & $<3.0$ \\
\hline \multirow[t]{2}{*}{$27 \mathrm{cda}$} & $08-25-86$ & $<3.0$ & $<3.0$ & $<3.0$ & $<3.0$ & $<3.0$ & $<3.0$ & $<3.0$ & $<3.0$ \\
\hline & $03-30-89$ & $<3.0$ & $<3.0$ & $<3.0$ & $<3.0$ & $<3.0$ & $<3.0$ & $<3.0$ & $<3.0$ \\
\hline \multirow[t]{2}{*}{ 26dda2 } & $08-26-86$ & $<3.0$ & $<3.0$ & $<3.0$ & $<3.0$ & $<3.0$ & $<3.0$ & $<3.0$ & $<3.0$ \\
\hline & $03-29-89$ & $<3.0$ & $<3.0$ & $<3.0$ & $<3.0$ & $<3.0$ & $<3.0$ & $<3.0$ & $<3.0$ \\
\hline \multirow[t]{2}{*}{$26 \mathrm{dac} 2$} & $08-26-86$ & $<3.0$ & $<3.0$ & $<3.0$ & $<3.0$ & $<3.0$ & $<3.0$ & $<3.0$ & $<3.0$ \\
\hline & $03-29-89$ & $<3.0$ & $<3.0$ & $<3.0$ & $<3.0$ & $<3.0$ & $<3.0$ & $<3.0$ & $<3.0$ \\
\hline \multirow[t]{2}{*}{$28 \mathrm{dad}$} & $08-25-86$ & $<3.0$ & $<3.0$ & $<3.0$ & $<3.0$ & $<3.0$ & $<3.0$ & $<3.0$ & $<3.0$ \\
\hline & $03-29-89$ & $<3.0$ & $<3.0$ & $<3.0$ & $<3.0$ & $<3.0$ & $<3.0$ & $<3.0$ & $<3.0$ \\
\hline \multirow[t]{2}{*}{ 27ada2 } & $08-27-86$ & $<3.0$ & $<3.0$ & $<3.0$ & $<3.0$ & $<3.0$ & $<3.0$ & $<3.0$ & $<3.0$ \\
\hline & $03-29-89$ & $<3.0$ & $<3.0$ & $<3.0$ & $<3.0$ & $<3.0$ & $<3.0$ & $<3.0$ & $<3.0$ \\
\hline \multirow[t]{3}{*}{$27 \mathrm{bdb} 4$} & $09-08-86$ & $<3.0$ & $<3.0$ & $<3.0$ & $<3.0$ & $<3.0$ & $<3.0$ & $<3.0$ & $<3.0$ \\
\hline & $06-15-87$ & $<3.0$ & $<3.0$ & $<3.0$ & $<3.0$ & $<3.0$ & $<3.0$ & $<3.0$ & $<3.0$ \\
\hline & $03-27-89$ & $<3.0$ & $<3.0$ & $<3.0$ & $<3.0$ & $<3.0$ & $<3.0$ & $<3.0$ & $<3.0$ \\
\hline \multirow[t]{3}{*}{$26 \mathrm{bbb}$} & $09-08-86$ & $<3.0$ & $<3.0$ & $<3.0$ & $<3.0$ & $<3.0$ & $<3.0$ & $<3.0$ & $<3.0$ \\
\hline & $06-15-87$ & $<3.0$ & $<3.0$ & $<3.0$ & $<3.0$ & $<3.0$ & $<3.0$ & $<3.0$ & $<3.0$ \\
\hline & $03-27-89$ & $<3.0$ & $<3.0$ & $<3.0$ & $<3.0$ & $<3.0$ & $<3.0$ & $<3.0$ & $<3.0$ \\
\hline $26 \mathrm{dcb} 2$ & $03-28-89$ & $<3.0$ & $<3.0$ & $<3.0$ & $<3.0$ & $<3.0$ & $<3.0$ & $<3.0$ & $<3.0$ \\
\hline $26 \mathrm{cbb} 2$ & $03-28-89$ & $<3.0$ & $<3.0$ & $<3.0$ & $<3.0$ & $<3.0$ & $<3.0$ & $<3.0$ & $<3.0$ \\
\hline $26 \mathrm{cbb3}$ & $03-28-89$ & $<3.0$ & $<3.0$ & $<3.0$ & $<3.0$ & $<3.0$ & $<3.0$ & $<3.0$ & $<3.0$ \\
\hline
\end{tabular}


Table 9. Analytical results of ground-water samples, Rillito Creek basin-Continued Volatile organic compounds-Continued

\begin{tabular}{|c|c|c|c|c|c|c|c|c|c|c|}
\hline $\begin{array}{c}\text { Well } \\
\text { number } \\
(D-13-14)\end{array}$ & Date & $\begin{array}{c}\text { Di- } \\
\text { chioro- } \\
\text { bromo- } \\
\text { methane, } \\
\text { total } \\
(\mu \mathrm{g} / \mathrm{L})\end{array}$ & $\begin{array}{c}\text { Di- } \\
\text { chiorodi- } \\
\text { fluoro- } \\
\text { methane, } \\
\text { total } \\
(\mu g / L)\end{array}$ & $\begin{array}{c}\text { 1,1-Di- } \\
\text { chioro- } \\
\text { ethane, } \\
\text { total } \\
(\mu g / L)\end{array}$ & $\begin{array}{c}\text { 1,2-Di- } \\
\text { chioro- } \\
\text { ethane, } \\
\text { total } \\
(\mu g / L)\end{array}$ & $\begin{array}{c}\text { 1,1-Di- } \\
\text { chloro- } \\
\text { ethylene, } \\
\text { total } \\
(\mu \mathrm{g} / \mathrm{L})\end{array}$ & $\begin{array}{c}\text { 1,2- } \\
\text { Transdi- } \\
\text { chioro- } \\
\text { ethane, } \\
\text { total } \\
(\mu \mathrm{g} / \mathrm{L})\end{array}$ & $\begin{array}{c}\text { 1,2-Di- } \\
\text { chioro- } \\
\text { propane, } \\
\text { total } \\
(\mu g / L)\end{array}$ & $\begin{array}{c}1,3- \\
\text { Dichioro- } \\
\text { propene, } \\
\text { total } \\
(\mu g / L)\end{array}$ & $\begin{array}{c}\text { Ethyi- } \\
\text { benzene, } \\
\text { total } \\
(\mu \mathrm{g} / \mathrm{L})\end{array}$ \\
\hline \multirow[t]{3}{*}{$34 \mathrm{dcc} 2$} & $09-08-86$ & $<3.0$ & $<3.0$ & $<3.0$ & $<3.0$ & $<3.0$ & $<3.0$ & $<3.0$ & $<3.0$ & $<3.0$ \\
\hline & $06-16-87$ & $<3.0$ & $<3.0$ & $<3.0$ & $<3.0$ & $<3.0$ & $<3.0$ & $<3.0$ & $<3.0$ & $<3.0$ \\
\hline & $03-27-89$ & $<3.0$ & $<3.0$ & $<3.0$ & $<3.0$ & $<3.0$ & $<3.0$ & $<3.0$ & $<3.0$ & $<3.0$ \\
\hline 34aaa1 & $03-29-89$ & $<3.0$ & $<3.0$ & $<3.0$ & $<3.0$ & $<3.0$ & $<3.0$ & $<3.0$ & $<3.0$ & $<3.0$ \\
\hline \multirow[t]{2}{*}{$27 \mathrm{ddc} 3$} & $08-27-86$ & $<3.0$ & $<3.0$ & $<3.0$ & $<3.0$ & $<3.0$ & $<3.0$ & $<3.0$ & $<3.0$ & $<3.0$ \\
\hline & $03-28-89$ & $<3.0$ & $<3.0$ & $<3.0$ & $<3.0$ & $<3.0$ & $<3.0$ & $<3.0$ & $<3.0$ & $<3.0$ \\
\hline $26 \mathrm{cdd}$ & $09-09-86$ & $<3.0$ & $<3.0$ & $<3.0$ & $<3.0$ & $<3.0$ & $<3.0$ & $<3.0$ & $<3.0$ & $<3.0$ \\
\hline \multirow[t]{2}{*}{$27 \mathrm{cda}$} & $08-25-86$ & $<3.0$ & $<3.0$ & $<3.0$ & $<3.0$ & $<3.0$ & $<3.0$ & $<3.0$ & $<3.0$ & $<3.0$ \\
\hline & $03-30-89$ & $<3.0$ & $<3.0$ & $<3.0$ & $<3.0$ & $<3.0$ & $<3.0$ & $<3.0$ & $<3.0$ & $<3.0$ \\
\hline \multirow[t]{2}{*}{ 26dda2 } & $08-26-86$ & $<3.0$ & $<3.0$ & $<3.0$ & $<3.0$ & $<3.0$ & $<3.0$ & $<3.0$ & $<3.0$ & $<3.0$ \\
\hline & $03-29-89$ & $<3.0$ & $<3.0$ & $<3.0$ & $<3.0$ & $<3.0$ & $<3.0$ & $<3.0$ & $<3.0$ & $<3.0$ \\
\hline \multirow[t]{2}{*}{26 dac2 } & $08-26-86$ & $<3.0$ & $<3.0$ & $<3.0$ & $<3.0$ & $<3.0$ & $<3.0$ & $<3.0$ & $<3.0$ & $<3.0$ \\
\hline & $03-29-89$ & $<3.0$ & $<3.0$ & $<3.0$ & $<3.0$ & $<3.0$ & $<3.0$ & $<3.0$ & $<3.0$ & $<3.0$ \\
\hline \multirow[t]{2}{*}{28 dad } & $08-25-86$ & $<3.0$ & $<3.0$ & $<3.0$ & $<3.0$ & $<3.0$ & $<3.0$ & $<3.0$ & $<3.0$ & $<3.0$ \\
\hline & $03-29-89$ & $<3.0$ & $<.0$ & $<3.0$ & $<3.0$ & $<3.0$ & $<3.0$ & $<3.0$ & $<3.0$ & $<3.0$ \\
\hline \multirow[t]{2}{*}{ 27ada2 } & $08-27-86$ & $<3.0$ & $<3.0$ & $<3.0$ & $<3.0$ & $<3.0$ & $<3.0$ & $<3.0$ & $<3.0$ & $<3.0$ \\
\hline & $03-29-89$ & $<3.0$ & $<3.0$ & $<3.0$ & $<3.0$ & $<3.0$ & $<3.0$ & $<3.0$ & $<3.0$ & $<3.0$ \\
\hline \multirow[t]{3}{*}{ 27bdb4 } & $09-08-86$ & $<3.0$ & $<3.0$ & $<3.0$ & $<3.0$ & $<3.0$ & $<3.0$ & $<3.0$ & $<3.0$ & $<3.0$ \\
\hline & $06-15-87$ & $<3.0$ & $<3.0$ & $<3.0$ & $<3.0$ & $<3.0$ & $<3.0$ & $<3.0$ & $<3.0$ & $<3.0$ \\
\hline & $03-27-89$ & $<3.0$ & $<3.0$ & $<3.0$ & $<3.0$ & $<3.0$ & $<3.0$ & $<3.0$ & $<3.0$ & $<3.0$ \\
\hline \multirow[t]{3}{*}{$26 \mathrm{bbb}$} & $09-08-86$ & $<3.0$ & $<3.0$ & $<3.0$ & $<3.0$ & $<3.0$ & $<3.0$ & $<3.0$ & $<3.0$ & $<3.0$ \\
\hline & $06-15-87$ & $<3.0$ & $<3.0$ & $<3.0$ & $<3.0$ & $<3.0$ & $<3.0$ & $<3.0$ & $<3.0$ & $<3.0$ \\
\hline & $03-27-89$ & $<3.0$ & $<3.0$ & $<3.0$ & $<3.0$ & $<3.0$ & $<3.0$ & $<3.0$ & $<3.0$ & $<3.0$ \\
\hline $26 \mathrm{dcb} 2$ & $03-28-89$ & $<3.0$ & $<3.0$ & $<3.0$ & $<3.0$ & $<3.0$ & $<3.0$ & $<3.0$ & $<3.0$ & $<3.0$ \\
\hline $26 \mathrm{cbb} 2$ & $03-28-89$ & $<3.0$ & $<3.0$ & $<3.0$ & $<3.0$ & $<3.0$ & $<3.0$ & $<3.0$ & $<3.0$ & $<3.0$ \\
\hline $26 \mathrm{cbb3}$ & $03-28-89$ & $<3.0$ & $<3.0$ & $<3.0$ & $<3.0$ & $<3.0$ & $<3.0$ & $<3.0$ & $<3.0$ & $<3.0$ \\
\hline
\end{tabular}


Table 9. Analytical results of ground-water samples, Rillito Creek basin-Continued Volatile organic compounds-Continued

\begin{tabular}{|c|c|c|c|c|c|c|c|c|c|c|}
\hline $\begin{array}{c}\text { Well } \\
\text { number } \\
\text { (D-13-14) }\end{array}$ & Date & $\begin{array}{c}\text { Methyl- } \\
\text { bromide, } \\
\text { total } \\
(\mu \mathrm{g} / \mathrm{L})\end{array}$ & $\begin{array}{c}\text { Methyl- } \\
\text { one, } \\
\text { chloride, } \\
\text { total } \\
(\mu g / L)\end{array}$ & $\begin{array}{c}1,1,2,2- \\
\text { Tetra- } \\
\text { chloro- } \\
\text { ethane, } \\
\text { total } \\
(\mu g / L)\end{array}$ & $\begin{array}{l}\text { Tetra- } \\
\text { chloro- } \\
\text { ethyl- } \\
\text { ene, } \\
\text { total } \\
(\mu \mathrm{g} / \mathrm{L})\end{array}$ & $\begin{array}{c}\text { Toluene, } \\
\text { total } \\
(\mu g / L)\end{array}$ & $\begin{array}{c}1,1,1-T r 1- \\
\text { chloro- } \\
\text { ethane, } \\
\text { total } \\
(\mu g / L)\end{array}$ & $\begin{array}{c}\text { 1,1,2- } \\
\text { Trlchloro- } \\
\text { ethane, } \\
\text { total } \\
(\mu g / L)\end{array}$ & $\begin{array}{c}\text { Trl- } \\
\text { chloro- } \\
\text { ethylene, } \\
\text { total } \\
(\mu g / L)\end{array}$ & $\begin{array}{c}\text { Tri- } \\
\text { chloro- } \\
\text { fluoro- } \\
\text { methane, } \\
\text { total } \\
(\mu \mathrm{g} / \mathrm{L})\end{array}$ \\
\hline \multirow[t]{3}{*}{$34 \mathrm{dcc} 2$} & $09-08-86$ & 5.3 & $<3.0$ & $<3.0$ & $<3.0$ & $<3.0$ & $<3.0$ & $<3.0$ & $<3.0$ & $<3.0$ \\
\hline & $06-16-87$ & $<3.0$ & $<3.0$ & $<3.0$ & $<3.0$ & $<3.0$ & $<3.0$ & $<3.0$ & $<3.0$ & $<3.0$ \\
\hline & $03-27-89$ & $<3.0$ & $<3.0$ & $<3.0$ & $<3.0$ & $<3.0$ & $<3.0$ & $<3.0$ & $<3.0$ & $<3.0$ \\
\hline 34aaa1 & $03-29-89$ & $<3.0$ & $<3.0$ & $<3.0$ & $<3.0$ & $<3.0$ & $<3.0$ & $<3.0$ & $<3.0$ & $<3.0$ \\
\hline \multirow[t]{2}{*}{$27 \mathrm{ddc} 3$} & $08-27-86$ & $<3.0$ & $<3.0$ & $<3.0$ & $<3.0$ & $<3.0$ & $<3.0$ & $<3.0$ & $<3.0$ & $<3.0$ \\
\hline & $03-28-89$ & $<3.0$ & $<3.0$ & $<3.0$ & $<3.0$ & $<3.0$ & $<3.0$ & $<3.0$ & $<3.0$ & $<3.0$ \\
\hline $26 \mathrm{cdd}$ & $09-09-86$ & $<3.0$ & $<3.0$ & $<3.0$ & $<3.0$ & $<3.0$ & $<3.0$ & $<3.0$ & $<3.0$ & $<3.0$ \\
\hline \multirow[t]{2}{*}{$27 \mathrm{cda}$} & $08-25-86$ & $<3.0$ & $<3.0$ & $<3.0$ & $<3.0$ & $<3.0$ & $<3.0$ & $<3.0$ & $<3.0$ & $<3.0$ \\
\hline & $03-30-89$ & $<3.0$ & $<3.0$ & $<3.0$ & $<3.0$ & $<3.0$ & $<3.0$ & $<3.0$ & $<3.0$ & $<3.0$ \\
\hline \multirow[t]{2}{*}{ 26dda2 } & $08-26-86$ & $<3.0$ & $<3.0$ & $<3.0$ & $<3.0$ & $<3.0$ & $<3.0$ & $<3.0$ & $<3.0$ & $<3.0$ \\
\hline & $03-29-89$ & $<3.0$ & $<3.0$ & $<3.0$ & $<3.0$ & $<3.0$ & $<3.0$ & $<3.0$ & $<3.0$ & $<3.0$ \\
\hline \multirow[t]{2}{*}{26 dac2 } & $08-26-86$ & $<3.0$ & $<3.0$ & $<3.0$ & $<3.0$ & $<3.0$ & $<3.0$ & $<3.0$ & $<3.0$ & $<3.0$ \\
\hline & $03-29-89$ & $<3.0$ & $<3.0$ & $<3.0$ & $<3.0$ & $<3.0$ & $<3.0$ & $<3.0$ & $<3.0$ & $<3.0$ \\
\hline \multirow[t]{2}{*}{ 28dad } & $08-25-86$ & $<3.0$ & $<3.0$ & $<3.0$ & $<3.0$ & $<3.0$ & $<3.0$ & $<3.0$ & $<3.0$ & $<3.0$ \\
\hline & $03-29-89$ & $<3.0$ & $<3.0$ & $<3.0$ & $<3.0$ & $<3.0$ & $<3.0$ & $<3.0$ & $<3.0$ & $<3.0$ \\
\hline \multirow[t]{2}{*}{ 27ada2 } & $08-27-86$ & $<3.0$ & $<3.0$ & $<3.0$ & $<3.0$ & $<3.0$ & $<3.0$ & $<3.0$ & $<3.0$ & $<3.0$ \\
\hline & $03-29-89$ & $<3.0$ & $<3.0$ & $<3.0$ & $<3.0$ & $<3.0$ & $<3.0$ & $<3.0$ & $<3.0$ & $<3.0$ \\
\hline \multirow[t]{3}{*}{ 27bdb4 } & $09-08-86$ & 3.0 & $<3.0$ & $<3.0$ & $<3.0$ & $<3.0$ & $<3.0$ & $<3.0$ & $<3.0$ & $<3.0$ \\
\hline & $06-15-87$ & $<3.0$ & $<3.0$ & $<3.0$ & $<3.0$ & $<3.0$ & $<3.0$ & $<3.0$ & $<3.0$ & $<3.0$ \\
\hline & $03-27-89$ & $<3.0$ & $<3.0$ & $<3.0$ & $<3.0$ & $<3.0$ & $<3.0$ & $<3.0$ & $<3.0$ & $<3.0$ \\
\hline \multirow[t]{3}{*}{$26 \mathrm{bbb}$} & $09-08-86$ & $<3.0$ & $<3.0$ & $<3.0$ & $<3.0$ & 3.2 & $<3.0$ & $<3.0$ & $<3.0$ & $<3.0$ \\
\hline & $06-15-87$ & $<3.0$ & $<3.0$ & $<3.0$ & $<3.0$ & $<3.0$ & $<3.0$ & $<3.0$ & $<3.0$ & $<3.0$ \\
\hline & $03-27-89$ & $<3.0$ & $<3.0$ & $<3.0$ & $<3.0$ & $<3.0$ & $<3.0$ & $<3.0$ & $<3.0$ & $<3.0$ \\
\hline $26 \mathrm{dcb} 2$ & $03-28-89$ & $<3.0$ & $<3.0$ & $<3.0$ & $<3.0$ & $<3.0$ & $<3.0$ & $<3.0$ & $<3.0$ & $<3.0$ \\
\hline $26 \mathrm{cbb} 2$ & $03-28-89$ & $<3.0$ & $<3.0$ & $<3.0$ & $<3.0$ & $<3.0$ & $<3.0$ & $<3.0$ & $<3.0$ & $<3.0$ \\
\hline $26 \mathrm{cbb} 3$ & $03-28-89$ & $<3.0$ & $<3.0$ & $<3.0$ & $<3.0$ & $<3.0$ & $<3.0$ & $<3.0$ & $<3.0$ & $<3.0$ \\
\hline
\end{tabular}


Table 9. Analytical results of ground-water samples, Rillito Creek basin-Continued Volatile organic compounds-Continued

\begin{tabular}{|c|c|c|c|c|c|c|c|c|c|c|}
\hline $\begin{array}{c}\text { Well } \\
\text { number } \\
\text { (D-13-14) }\end{array}$ & Date & $\begin{array}{c}\text { Vinyl } \\
\text { chlo- } \\
\text { ride, } \\
\text { total } \\
(\mu g / L)\end{array}$ & $\begin{array}{c}\text { Methyl- } \\
\text { chloride, } \\
\text { total } \\
(\mu \mathrm{g} / \mathrm{L})\end{array}$ & $\begin{array}{c}\text { 1,2-Di- } \\
\text { bromo- } \\
\text { ethane, } \\
\text { total } \\
(\mu \mathrm{g} / \mathrm{L})\end{array}$ & $\begin{array}{c}\text { 1,2-Di- } \\
\text { chloro- } \\
\text { benzene, } \\
\text { total } \\
(\mu g / L)\end{array}$ & $\begin{array}{c}\text { 1,3-Di- } \\
\text { chloro- } \\
\text { benzene, } \\
\text { total } \\
(\mu \mathrm{g} / \mathrm{L})\end{array}$ & $\begin{array}{c}\text { 1,4-Di- } \\
\text { chloro- } \\
\text { benzene, } \\
\text { total } \\
(\mu g / L)\end{array}$ & $\begin{array}{c}\text { Cls- } \\
\text { 1,3-Di- } \\
\text { chloro- } \\
\text { propene, } \\
\text { total } \\
(\mu g / L)\end{array}$ & $\begin{array}{c}\text { Trans- } \\
\text { 1,3-Di- } \\
\text { chloro- } \\
\text { propene, } \\
\text { total } \\
(\mu \mathrm{g} / \mathrm{L})\end{array}$ & $\begin{array}{c}\text { Xylene, } \\
\text { total } \\
(\mu g / L)\end{array}$ \\
\hline \multirow[t]{3}{*}{$34 \mathrm{dcc} 2$} & $09-08-86$ & $<3.0$ & 7.7 & $\cdots$ & $<3.0$ & $<3.0$ & $<3.0$ & $<3.0$ & $<3.0$ & $<3.0$ \\
\hline & $06-16-87$ & $<3.0$ & $<3.0$ & --- & $<5.0$ & $<5.0$ & $<5.0$ & $<3.0$ & $<3.0$ & $<3.0$ \\
\hline & $03-27-89$ & $<1.0$ & $<3.0$ & $<3.0$ & $<5.0$ & $<5.0$ & $<5.0$ & $<3.0$ & $<3.0$ & $<3.0$ \\
\hline 34aaa1 & $03-29-89$ & $<1.0$ & $<3.0$ & $<3.0$ & $<3.0$ & $<3.0$ & $<3.0$ & $<3.0$ & $<3.0$ & $<3.0$ \\
\hline \multirow[t]{2}{*}{$27 \mathrm{ddc} 3$} & $08-27-86$ & $<3.0$ & $<3.0$ & -- & $<3.0$ & $<3.0$ & $<3.0$ & $<3.0$ & $<3.0$ & $<3.0$ \\
\hline & $03-28-89$ & $<1.0$ & $<3.0$ & $<3.0$ & $<3.0$ & $<3.0$ & $<3.0$ & $<3.0$ & $<3.0$ & $<3.0$ \\
\hline $26 \mathrm{cdd}$ & $09-09-86$ & $<3.0$ & $<3.0$ & $-\cdots$ & $<3.0$ & $<3.0$ & $<3.0$ & $<3.0$ & $<3.0$ & -.. \\
\hline \multirow[t]{2}{*}{$27 \mathrm{cda}$} & $08-25-86$ & $<3.0$ & $<3.0$ & -- & $<3.0$ & $<3.0$ & $<3.0$ & $<3.0$ & $<3.0$ & $<3.0$ \\
\hline & 03-30-89 & $<1.0$ & $<3.0$ & $<3.0$ & $<3.0$ & $<3.0$ & $<3.0$ & $<3.0$ & $<3.0$ & $<3.0$ \\
\hline \multirow[t]{2}{*}{ 26dda2 } & $08-26-86$ & $<3.0$ & $<3.0$ & --- & $<3.0$ & $<3.0$ & $<3.0$ & $<3.0$ & $<3.0$ & $<3.0$ \\
\hline & $03-29-89$ & $<1.0$ & $<3.0$ & $<3.0$ & $<3.0$ & $<3.0$ & $<3.0$ & $<3.0$ & $<3.0$ & $<3.0$ \\
\hline \multirow[t]{2}{*}{ 26dac2 } & $08-26-86$ & $<3.0$ & $<3.0$ & --- & $<3.0$ & $<3.0$ & $<3.0$ & $<3.0$ & $<3.0$ & $<3.0$ \\
\hline & $03-29-89$ & $<1.0$ & $<3.0$ & $<3.0$ & $<5.0$ & $<5.0$ & $<5.0$ & $<3.0$ & $<3.0$ & $<3.0$ \\
\hline \multirow[t]{2}{*}{$28 \mathrm{dad}$} & $08-25-86$ & $<3.0$ & $<3.0$ & -- & $<3.0$ & $<3.0$ & $<3.0$ & $<3.0$ & $<3.0$ & $<3.0$ \\
\hline & $03-29-89$ & $<1.0$ & $<3.0$ & $<3.0$ & $<3.0$ & $<3.0$ & $<3.0$ & $<3.0$ & $<3.0$ & $<3.0$ \\
\hline \multirow[t]{2}{*}{ 27ada2 } & $08-27-86$ & $<3.0$ & $<3.0$ & $\cdots$ & $<3.0$ & $<3.0$ & $<3.0$ & $<3.0$ & $<3.0$ & $<3.0$ \\
\hline & $03-29-89$ & $<1.0$ & $<3.0$ & $<3.0$ & $<3.0$ & $<3.0$ & $<3.0$ & $<3.0$ & $<3.0$ & $<3.0$ \\
\hline \multirow[t]{3}{*}{$27 \mathrm{bdb} 4$} & $09-08-86$ & $<3.0$ & 8.0 & -- & $<3.0$ & $<3.0$ & $<3.0$ & $<3.0$ & $<3.0$ & -- \\
\hline & $06-15-87$ & $<3.0$ & $<3.0$ & --- & $<5.0$ & $<5.0$ & $<5.0$ & $<3.0$ & $<3.0$ & $<3.0$ \\
\hline & $03-27-89$ & $<1.0$ & $<3.0$ & $<3.0$ & $<5.0$ & $<5.0$ & $<5.0$ & $<3.0$ & $<3.0$ & $<3.0$ \\
\hline \multirow[t]{3}{*}{$26 \mathrm{bbb}$} & $09-08-86$ & $<3.0$ & $<3.0$ & -- & $<3.0$ & $<3.0$ & $<3.0$ & $<3.0$ & $<3.0$ & $<3.0$ \\
\hline & $06-15-87$ & $<3.0$ & $<3.0$ &.-- & $<3.0$ & $<3.0$ & $<3.0$ & $<3.0$ & $<3.0$ & $<3.0$ \\
\hline & $03-27-89$ & $<1.0$ & $<3.0$ & $<3.0$ & $<3.0$ & $<3.0$ & $<3.0$ & $<3.0$ & $<3.0$ & $<3.0$ \\
\hline $26 \mathrm{dcb} 2$ & $03-28-89$ & $<1.0$ & $<3.0$ & $<3.0$ & $<3.0$ & $<3.0$ & $<3.0$ & $<3.0$ & $<3.0$ & $<3.0$ \\
\hline $26 \mathrm{cbb} 2$ & $03-28-89$ & $<1.0$ & $<3.0$ & $<3.0$ & $<3.0$ & $<3.0$ & $<3.0$ & $<3.0$ & $<3.0$ & $<3.0$ \\
\hline $26 \mathrm{cbb} 3$ & $03-28-89$ & $<1.0$ & $<3.0$ & $<3.0$ & $<3.0$ & $<3.0$ & $<3.0$ & $<3.0$ & $<3.0$ & $<3.0$ \\
\hline
\end{tabular}


Tabie 9. Analytical results of ground-water samples, Rillito Creek basin-Continued Organic carbon and oil and grease

\begin{tabular}{|c|c|c|c|c|}
\hline $\begin{array}{l}\text { Well number } \\
\text { (D-13-14) }\end{array}$ & Date & $\begin{array}{l}\text { Carbon organic, } \\
\text { dissolved } \\
\text { (mg/L as C) }\end{array}$ & $\begin{array}{l}\text { Carbon, organic total } \\
(\mathrm{mg} / \mathrm{L} \text { as } \mathrm{C})\end{array}$ & $\begin{array}{l}\text { Oll and grease, } \\
\text { total recoverable, } \\
\text { gravimetric (mg/L) }\end{array}$ \\
\hline \multirow[t]{2}{*}{$34 \mathrm{dcc} 2$} & $09-08-86$ & 3.9 & 3.9 & 0.3 \\
\hline & $03-27-89$ & -- & 1.0 & -- \\
\hline \multirow[t]{2}{*}{ 34aaal } & $09-09-86$ & 1.1 & 2.3 & .2 \\
\hline & $03-29-89$ & --- & .4 & -- \\
\hline \multirow[t]{2}{*}{$27 \mathrm{ddc} 3$} & $08-27-86$ & .9 & .7 & .2 \\
\hline & $03-28-89$ & --- & .3 & -- \\
\hline $26 \mathrm{cdd}$ & $09-09-86$ & 1.8 & 2.1 & .3 \\
\hline \multirow[t]{2}{*}{$27 \mathrm{cda}$} & $08-25-86$ & 1.3 & .3 & .2 \\
\hline & $03-30-89$ & --- & 1.0 & --- \\
\hline \multirow[t]{2}{*}{ 26dda2 } & $08-26-86$ & 2.6 & 2.3 & .1 \\
\hline & $03-29-89$ & --- & 1.0 & -- \\
\hline \multirow[t]{2}{*}{26 dac2 } & $08-26-86$ & 2.5 & 2.8 & .2 \\
\hline & $03-29-89$ & $\cdots$ & 1.1 & --- \\
\hline \multirow[t]{2}{*}{ 28dad } & $08-25-86$ & 1.3 & 1.1 & .2 \\
\hline & $03-29-89$ & -- & .7 & -- \\
\hline \multirow[t]{2}{*}{ 27ada2 } & $09-08-86$ & 1.0 & .9 & .2 \\
\hline & $03-27-89$ & --- & .1 & -- \\
\hline \multirow[t]{2}{*}{$27 \mathrm{bdb} 4$} & $09-08-86$ & 3.5 & 3.6 & .3 \\
\hline & $03-27-89$ & -- & 1.5 & -- \\
\hline $26 \mathrm{bbb}$ & $09-08-86$ & 3.5 & 4.0 & .2 \\
\hline $26 \mathrm{dcb} 2$ & $03-28-89$ & --- & 1.5 & $\cdots$ \\
\hline $26 \mathrm{cbb} 2$ & $03-28-89$ & --- & .7 & $\cdots$ \\
\hline $26 \mathrm{cbb} 3$ & $03-28-89$ & --. & 3.2 & -.. \\
\hline
\end{tabular}


Table 10. Particle-size distribution of bottom-sediment samples, Rillito Creek basin $[<$, less than; mm, millimeters; dashes indicate no data]

\begin{tabular}{|c|c|c|c|}
\hline \multirow[b]{2}{*}{ Date } & \multicolumn{3}{|c|}{ Particle-size distribution, in percent } \\
\hline & $\begin{array}{c}\text { Gravel } \\
(<2 \mathrm{~mm})\end{array}$ & $\begin{array}{c}\text { Sand } \\
(0.062 \text { to } 2 \mathrm{~mm})\end{array}$ & $\begin{array}{l}\text { Silt and clay } \\
(<0.062 \mathrm{~mm})\end{array}$ \\
\hline \multicolumn{4}{|c|}{ Tanque VerdeCree at Tucron } \\
\hline $07-24-89$ & 9.1 & 86.8 & 4.1 \\
\hline $07-21-92$ & -- & 99 & 1 \\
\hline \multicolumn{4}{|c|}{ Pantano Washat Broadway Boulevard } \\
\hline $02-21-92$ & -- & 96.4 & 3.6 \\
\hline \multicolumn{4}{|c|}{ Alamo Wash at Genn Street } \\
\hline $02-21-92$ & $\overline{---}$ & 99.5 & .5 \\
\hline \multicolumn{4}{|c|}{ Rilito Creek at Dodge Boulevard } \\
\hline $02-21-92$ & --- & 99.3 & .7 \\
\hline
\end{tabular}

Tabie 11. Analytical results of bottom-sediment samples, Rillito Creek basin Nutrients

[mg/kg, milligrams per kilogram; $\mu \mathrm{g} / \mathrm{g}$, micrograms per gram; $\mathrm{pCi} / \mathrm{g}$, picocuries per gram; $\mu \mathrm{g} / \mathrm{kg}$, micrograms per kilogram; g/kg, grams per kilogram; <, less than; dashes indicate no data]

\begin{tabular}{|c|c|c|c|c|c|}
\hline Date & $\begin{array}{l}\text { Moisture content, } \\
\text { dry weight } \\
\text { (percentage of total) }\end{array}$ & $\begin{array}{l}\text { Phosphorus, fotal } \\
\text { (mg/kg as P) }\end{array}$ & $\begin{array}{c}\text { Nitrogen, } \\
\mathrm{NO}_{2}+\mathrm{NO}_{3} \\
\text { total } \\
\text { (mg/kg as } \mathrm{N} \text { ) }\end{array}$ & $\begin{array}{c}\text { Nitrogen, } \mathrm{NH}_{4} \text {, total } \\
(\mathrm{mg} / \mathrm{kg} \text { as } \mathrm{N})\end{array}$ & $\begin{array}{l}\text { Nitrogen, } \mathrm{NH}_{4} \text { pius } \\
\text { organic, total } \\
\text { (mg/kg as } \mathrm{N} \text { ) }\end{array}$ \\
\hline \multicolumn{6}{|c|}{ Tanque Verde Creek at Tucson } \\
\hline $08-22-88$ & 3.0 & 210 & 3.0 & 15 & 240 \\
\hline $07-25-89$ & 26 & 160 & 26 & $<10$ & 450 \\
\hline $02-21-92$ & $<2.0$ & 240 & $<2.0$ & 2.3 & 50 \\
\hline \multicolumn{6}{|c|}{ Pantano Wash at Broadway Boulevard } \\
\hline $11-02-87$ & 2.0 & 160 & 2.0 & 1.4 & -- \\
\hline $02-20-92$ & $<2.0$ & 210 & $<2.0$ & .7 & 60 \\
\hline \multicolumn{6}{|c|}{ Alamo Wash at Glenn Street } \\
\hline $07-28-87$ & 12 & 190 & 12 & 11 & 230 \\
\hline $02-10-92$ & $<2.0$ & 170 & $<2.0$ & 1.7 & 80 \\
\hline \multicolumn{6}{|c|}{ Rillito Creek at Dodge Boulevard } \\
\hline $08-03-88$ & $<2.0$ & 160 & $<2.0$ & 5.3 & 200 \\
\hline $02-20-92$ & $<2.0$ & 220 & $<2.0$ & 1.1 & 40 \\
\hline
\end{tabular}


Table 11. Analytical results of bottom-sediment samples, Rillito Creek basin Trace elements

\begin{tabular}{|c|c|c|c|c|c|c|c|c|}
\hline Date & $\begin{array}{c}\text { Aluminum, } \\
\text { recover. } \\
\text { able } \\
\text { ( } \mu \mathrm{g} / \mathrm{g} \\
\text { as Al) }\end{array}$ & $\begin{array}{c}\text { Calcium, } \\
\text { recover- } \\
\text { able } \\
\text { ( } \mu \mathrm{g} / \mathrm{g} \\
\text { as Ca) }\end{array}$ & $\begin{array}{c}\text { Iron, } \\
\text { recover- } \\
\text { able } \\
(\mu \mathrm{g} / \mathrm{g} \\
\text { as Fe) }\end{array}$ & $\begin{array}{c}\text { Potassium, } \\
\text { recover- } \\
\text { able } \\
(\mu g / g \\
\text { as K) }\end{array}$ & $\begin{array}{c}\text { Magnesium, } \\
\text { recover- } \\
\text { able } \\
(\mu \mathrm{g} / \mathrm{g} \\
\text { as } \mathrm{Mg})\end{array}$ & $\begin{array}{c}\text { Sodium, } \\
\text { recover- } \\
\text { able } \\
(\mu g / g \\
\text { as } \mathrm{Na})\end{array}$ & $\begin{array}{c}\text { Titanium, } \\
\text { total } \\
(\mu \mathrm{g} / \mathrm{g} \\
\text { as } \mathrm{TI})\end{array}$ & $\begin{array}{c}\text { Manganese, } \\
\text { recover- } \\
\text { able } \\
\text { ( } \mu \mathrm{g} / \mathrm{g} \\
\text { as Mn) }\end{array}$ \\
\hline \multicolumn{9}{|c|}{ Tanque Verde Creekat Tueson } \\
\hline $08-22-88$ & 74,000 & 30,000 & 25,000 & 26,000 & 7,000 & 19,000 & 3,100 & 580 \\
\hline 07-25-89 & 76,300 & 34,600 & 27,600 & 24,600 & 8,600 & 15,000 & 3,300 & 1,180 \\
\hline $02-21-92$ & 65,000 & 8,400 & 4,200 & 32,000 & 800 & 24,000 & 500 & 180 \\
\hline \multicolumn{9}{|c|}{ Pantano Wash at Broadway Boulevard } \\
\hline $11-02-87$ & 63,000 & 39,000 & 24,000 & 21,000 & 7,400 & 17,000 & 2,800 & 520 \\
\hline $02-20-92$ & 54,000 & 14,000 & 8,500 & 29,000 & 2,900 & 17,000 & 1,100 & 200 \\
\hline \multicolumn{9}{|c|}{ Alamo Wash at Glenn Stred } \\
\hline $07-28-87$ & 65,000 & 47,000 & 26,000 & 24,000 & 9,500 & 12,000 & 3,200 & 540 \\
\hline $02-10-92$ & 54,000 & 12,000 & 6,900 & 31,000 & 1,400 & 17,000 & 900 & 260 \\
\hline \multicolumn{9}{|c|}{ Rilito Creek at Dodge Boulevard } \\
\hline $08-03-88$ & 66,000 & 56,000 & 27,000 & 24,000 & 9,300 & 13,000 & 2,800 & 630 \\
\hline $02-20-92$ & 64,000 & 12,000 & 6,900 & 29,000 & 1,400 & 23,000 & 800 & 240 \\
\hline
\end{tabular}

\begin{tabular}{|c|c|c|c|c|c|c|c|c|}
\hline Date & $\begin{array}{c}\text { Sllver, } \\
\text { recover- } \\
\text { able } \\
\text { ( } \mu \mathrm{g} / \mathrm{g} \\
\text { as } \mathrm{Ag} \text { ) }\end{array}$ & $\begin{array}{c}\text { Arsenlc, } \\
\text { total } \\
(\mu g / g \\
\text { as As) }\end{array}$ & $\begin{array}{c}\text { Gold, } \\
\text { sedIment } \\
\text { suspended } \\
(\mu g / g \\
\text { as Au) }\end{array}$ & $\begin{array}{c}\text { Barlum, } \\
\text { recoverable } \\
(\mu \mathrm{g} / \mathrm{g} \\
\text { as } \mathrm{Ba})\end{array}$ & $\begin{array}{c}\text { Beryllium, } \\
\text { recover- } \\
\text { able } \\
(\mu \mathrm{g} / \mathrm{g} \\
\text { as } \mathrm{Be})\end{array}$ & $\begin{array}{l}\text { Cadmlum, } \\
\text { recover- } \\
\text { able } \\
\text { ( } \mu \mathrm{g} / \mathrm{g} \\
\text { as Cd) }\end{array}$ & $\begin{array}{c}\text { Cobalt, } \\
\text { recoverable } \\
(\mu g / g \\
\text { as Co) }\end{array}$ & $\begin{array}{c}\text { Chromlum, } \\
\text { recoverable } \\
\text { ( } \mu \mathrm{g} / \mathrm{g} \\
\text { as } \mathrm{Cr} \text { ) }\end{array}$ \\
\hline \multicolumn{9}{|c|}{ Tanque Verde Creek at Tucson } \\
\hline $08-22-88$ & $<2$ & $<10$ & $<8$ & 750 & 2 & $<2$ & 8 & 33 \\
\hline $07-25-89$ & $<2$ & $<10$ & $<8$ & 676 & 2 & $<2$ & 11 & 34 \\
\hline $02-21-92$ & $<2$ & $<10$ & $<8$ & 1,000 & 2 & $<2$ & 2 & 3 \\
\hline \multicolumn{9}{|c|}{ Pantano Wash at Broadway Boulevard } \\
\hline $11-02-87$ & $<2$ & $<10$ & $<8$ & 650 & 2 & $<2$ & 8 & 51 \\
\hline $02-20-92$ & $<2$ & $<10$ & $<8$ & 750 & 1 & $<2$ & 3 & 5 \\
\hline \multicolumn{9}{|c|}{ Alamo Wash at Glenn Stred } \\
\hline $07-28-87$ & $<2$ & 8 & $<8$ & 700 & 2 & $<2$ & 9 & 44 \\
\hline $02-10-92$ & $<2$ & $<10$ & $<8$ & 830 & 1 & $<2$ & 3 & 6 \\
\hline \multicolumn{9}{|c|}{ Rilito Creek at Dodge Boulevard } \\
\hline $08-03-88$ & $<2$ & $<10$ & $<8$ & 700 & 2 & $<2$ & 9 & 30 \\
\hline $02-20-92$ & $<2$ & $<10$ & $<8$ & 860 & 1 & $<2$ & 2 & 5 \\
\hline
\end{tabular}


Table 11. Analytical results of bottom-sediment samples, Rillito Creek basin-Continued Trace elements-Continued

\begin{tabular}{|c|c|c|c|c|c|c|c|}
\hline Date & $\begin{array}{c}\text { Copper, } \\
\text { recoverable } \\
\text { ( } \mu \mathrm{g} / \mathrm{g} \text { as Cu) }\end{array}$ & $\begin{array}{l}\text { Lithium, } \\
\text { recoverable } \\
\text { ( } \mu \text { g/g as Li) }\end{array}$ & $\begin{array}{l}\text { Molybdenum, } \\
\text { recoverable } \\
\text { ( } \mu \mathrm{g} / \mathrm{g} \text { as Mo) }\end{array}$ & $\begin{array}{c}\text { Nickel, } \\
\text { recoverable } \\
\text { ( } \mu \mathrm{g} / \mathrm{g} \text { as Ni) }\end{array}$ & $\begin{array}{c}\text { Lead, } \\
\text { recoverable } \\
\text { ( } \mu \mathrm{g} / \mathrm{g} \text { as Pb) }\end{array}$ & $\begin{array}{c}\text { Scandium, } \\
\text { total } \\
(\mu \mathrm{g} / \mathrm{g} \text { as Sc) }\end{array}$ & $\begin{array}{c}\text { Thn, } \\
\text { recoverable } \\
\text { ( } \mu \mathrm{g} / \mathrm{g} \text { as } \mathrm{Sn})\end{array}$ \\
\hline \multicolumn{8}{|c|}{ Tanque Verde Creek at Tucson } \\
\hline $08-22-88$ & 30 & 30 & $<2$ & 10 & 40 & 80 & $<10$ \\
\hline $07-25-89$ & 80 & 36 & 2 & 19 & 58 & 11 & $<10$ \\
\hline $02-21-92$ & 4 & 9 & $<1$ & $<2$ & 25 & $<2$ & $<5$ \\
\hline \multicolumn{8}{|c|}{ Pantano Wash at Broadway Boulevard } \\
\hline $11-02-87$ & 30 & 30 & $<2$ & 20 & 30 & 70 & 180 \\
\hline $02-20-92$ & 9 & 16 & $<2$ & 3 & 20 & 2 & $<5$ \\
\hline \multicolumn{8}{|c|}{ Alamo Wash at Glenn Street } \\
\hline $07-28-87$ & 40 & 30 & $<2$ & 20 & 50 & 8 & $<10$ \\
\hline $02-10-92$ & 7 & 11 & $<2$ & 2 & 26 & $<2$ & $<5$ \\
\hline \multicolumn{8}{|c|}{ Rilito Creek at Dodge Boulevard } \\
\hline $08-03-88$ & 30 & 40 & $<2$ & 10 & 30 & 8 & $<10$ \\
\hline $02-20-92$ & 7 & 12 & $<2$ & 3 & 23 & 2 & $<5$ \\
\hline
\end{tabular}

\begin{tabular}{|c|c|c|c|c|c|c|c|}
\hline Date & $\begin{array}{l}\text { Tantalum, } \\
\text { ( } \mu \mathrm{g} / \mathrm{g} \text { as Ta) }\end{array}$ & $\begin{array}{c}\text { Thorium, } \\
\text { ( } \mu \mathrm{g} / \mathrm{g} \text { as } \mathrm{Th})\end{array}$ & $\begin{array}{c}\text { Uranium, } \\
\text { natural, totai } \\
(\mu \mathrm{g} / \mathrm{g} \text { as } \mathrm{U})\end{array}$ & $\begin{array}{c}\text { Vanadlum, } \\
\text { total } \\
\text { ( } \mu \mathrm{g} / \mathrm{g} \text { as } \mathrm{V})\end{array}$ & $\begin{array}{l}\text { Ytterbium, } \\
\text { ( } \mu \text { g/g as Yb) }\end{array}$ & $\begin{array}{c}\text { Zlnc, } \\
\text { recoverable } \\
\text { ( } \mu \mathrm{g} / \mathrm{g} \text { as Zn) }\end{array}$ & $\begin{array}{l}\text { Strontlum, } \\
\text { recoverable } \\
\text { ( } \mu \mathrm{g} / \mathrm{g} \text { as } \mathrm{Sr} \text { ) }\end{array}$ \\
\hline \multicolumn{8}{|c|}{ Tanque Verde Creek at Tucson } \\
\hline $08-22-88$ & $<40$ & 14 & $<100$ & 49 & 30 & 90 & 250 \\
\hline $07-25-89$ & $<40$ & 10 & $<100$ & 51 & 2 & 200 & 299 \\
\hline $02-21-92$ & $<40$ & $<4$ & $<100$ & 7 & $<1$ & 11 & 260 \\
\hline \multicolumn{8}{|c|}{ Pantano Wash at Broadway Boulevard } \\
\hline $110-02-87$ & $<40$ & 14 & $<100$ & 52 & 30 & 60 & 240 \\
\hline $02-20-92$ & $<40$ & 5 & $<100$ & 17 & 1 & 19 & 210 \\
\hline \multicolumn{8}{|c|}{ Alamo Wash at Glenn Street } \\
\hline $07-28-87$ & $<40$ & 16 & $<100$ & 64 & 30 & 100 & 260 \\
\hline $02-10-92$ & $<40$ & 5 & $<100$ & 14 & 1 & 23 & 200 \\
\hline \multicolumn{8}{|c|}{ Rillito Creek at Dodge Boulevard } \\
\hline $08-03-88$ & $<40$ & 14 & $<100$ & 59 & 30 & 100 & 250 \\
\hline $02-20-92$ & $<40$ & 4 & $<100$ & 12 & 1 & 16 & 250 \\
\hline
\end{tabular}


Table 11. Analytical results of bottom-sediment samples, Rillito Creek basin-Continued Radionuclides

\begin{tabular}{|c|c|c|c|c|c|c|}
\hline Date & $\begin{array}{c}\text { Gross alpha, } \\
\text { ( } \mu \mathrm{g} / \mathrm{g} \text { as U-Nat) }\end{array}$ & $\begin{array}{c}\text { Gross beta } \\
\text { (pCi/g as } \\
\text { Sr- } 90 / \mathrm{Y}-90 \text { ) }\end{array}$ & $\begin{array}{c}\text { Gross alpha, } \\
\text { (pCi/g as } \\
\text { Th-230) }\end{array}$ & $\begin{array}{c}\text { Gross beta, } \\
\text { (pCl/g as Cs-137) }\end{array}$ & $\begin{array}{l}\text { Uranium-235, } \\
\text { (pCi/g) }\end{array}$ & $\begin{array}{l}\text { Uranium-238, } \\
\qquad(\mathrm{pCl} / \mathrm{g})\end{array}$ \\
\hline \multicolumn{7}{|c|}{ Tanque Verde Creek at Tucson } \\
\hline $08-22-88$ & 14 & 34 & 8.9 & 54 & -- & -- \\
\hline $02-21-92$ & 9.4 & 5.1 & 6.7 & 9.8 & $-\ldots$ & $\cdots$ \\
\hline \multicolumn{7}{|c|}{ Pantano Wash at Broadway Boulevard } \\
\hline $11-02-87$ & 1.0 & 32 & .7 & 50 & .02 & .8 \\
\hline $02-20-92$ & 16.6 & 5.0 & 10.7 & 10.2 & --- & --- \\
\hline \multicolumn{7}{|c|}{ Alamo Wash at Glenn Street } \\
\hline $07-28-87$ & 14 & 35 & 10 & 59 & .06 & 1.10 \\
\hline $02-10-92$ & 11.6 & 33.8 & 17.1 & 9.4 & $--\infty$ & $\ldots$ \\
\hline \multicolumn{7}{|c|}{ Rfilito Creek at Dodge Boufevard } \\
\hline $08-03-88$ & 17 & 29 & 11 & 45 & $\cdots$ & $\cdots$ \\
\hline $02-20-92$ & 20.3 & 8.4 & 14.4 & 18 & $\cdots$ & -.. \\
\hline
\end{tabular}

Table 11. Analytical results of bottom-sediment samples, Rillito Creek basin-Continued Organochlorine pesticides

[DDD, dichlorodiphenyldichloroethane; DDE, dichlorodiphenylethylene; DDT, dichlorodiphenyltrichloroethane; PCB, polychlorinated biphenyl; PCN, polychlorinated naphthalene]

\begin{tabular}{|c|c|c|c|c|c|c|c|c|c|}
\hline Date & $\begin{array}{c}\text { Perthane, } \\
(\mu g / k g)\end{array}$ & $\begin{array}{c}\text { Endo- } \\
\text { sultan, } \\
\text { total } \\
(\mu \mathrm{g} / \mathrm{kg})\end{array}$ & $\begin{array}{c}\text { Aldrin, } \\
\text { total } \\
(\mu g / k g)\end{array}$ & $\begin{array}{c}\text { Chlordane, } \\
\text { total } \\
(\mu g / k g)\end{array}$ & $\begin{array}{c}\text { DDD, } \\
\text { total } \\
(\mu \mathrm{g} / \mathrm{kg})\end{array}$ & $\begin{array}{c}\text { DDE, } \\
\text { total } \\
(\mu \mathrm{g} / \mathrm{kg})\end{array}$ & $\begin{array}{c}\text { DDT, } \\
\text { total } \\
(\mu g / k g)\end{array}$ & $\begin{array}{c}\text { Dieldrin, } \\
\text { total } \\
(\mu \mathrm{g} / \mathrm{kg})\end{array}$ & $\begin{array}{c}\text { Endrin, } \\
\text { total } \\
(\mu \mathrm{g} / \mathrm{kg})\end{array}$ \\
\hline \multicolumn{10}{|c|}{ Tanque Verde Creek at Tucson } \\
\hline $08-22-88$ & $<1.0$ & $<0.1$ & $<0.1$ & 31 & $<0.1$ & $<0.1$ & 1.3 & 2.5 & $<0.1$ \\
\hline $02-21-92$ & $<1.0$ & $<.1$ & $<.1$ & $<1.0$ & $<.1$ & $<.1$ & $<.1$ & $<.1$ & $<.1$ \\
\hline \multicolumn{10}{|c|}{ Pantano Wash at Broadway Boulevard } \\
\hline $11-02-87$ & $<1.0$ & $<.1$ & $<.1$ & $<1.0$ & $<.1$ & $<.1$ & $<.1$ & $<.1$ & $<.1$ \\
\hline $02-20-92$ & $<1.0$ & $<.1$ & $<.1$ & $<1.0$ & $<.1$ & $<.1$ & $<.1$ & $<.1$ & $<1$ \\
\hline \multicolumn{10}{|c|}{ Alamo Wash at Glenn Street } \\
\hline $07-28-87$ & $<1.0$ & $<.1$ & $<.1$ & 140 & $<.1$ & 3.4 & 2.8 & 10 & $<.1$ \\
\hline $02-10-92$ & $<1.0$ & $<.1$ & $<.1$ & 1.0 & .1 & .2 & .2 & .7 & $<.1$ \\
\hline \multicolumn{10}{|c|}{ Rilito Creek at Dodge Boulevard } \\
\hline $08-03-88$ & $<1.0$ & $<.1$ & $<.1$ & 1.0 & $<.1$ & $<1.0$ & $<.1$ & .2 & $<.1$ \\
\hline $02-20-92$ & $<1.0$ & $<.1$ & $<.1$ & $<1.0$ & $<.1$ & $<1.0$ & $<.1$ & $<.1$ & $<.1$ \\
\hline
\end{tabular}

\begin{tabular}{|c|c|c|c|c|c|c|c|c|}
\hline Date & $\begin{array}{c}\text { Heptachlor, } \\
\text { total } \\
(\mu \mathrm{g} / \mathrm{kg})\end{array}$ & $\begin{array}{l}\text { Hepta- } \\
\text { chlor } \\
\text { epoxide, } \\
\text { total } \\
(\mu g / k g)\end{array}$ & $\begin{array}{l}\text { Lindane, } \\
\text { total } \\
(\mu \mathrm{g} / \mathrm{kg})\end{array}$ & $\begin{array}{c}\text { Toxaphene, } \\
\text { total } \\
(\mu \mathrm{g} / \mathrm{kg})\end{array}$ & $\begin{array}{l}\text { PCB, total } \\
(\mu \mathrm{g} / \mathrm{kg})\end{array}$ & $\begin{array}{l}\text { PCN, total } \\
(\mu \mathrm{g} / \mathbf{k g})\end{array}$ & $\begin{array}{c}\text { Methoxy- } \\
\text { chlor, total } \\
(\mu g / k g)\end{array}$ & $\begin{array}{c}\text { Mirex, total } \\
(\mu g / k g\end{array}$ \\
\hline \multicolumn{9}{|c|}{ Tanque Verde Creek at Tucson } \\
\hline $08-22-88$ & $<.1$ & $<.1$ & $<.1$ & $<10$ & 5 & $<1.0$ & $<.1$ & $<.1$ \\
\hline $02-21-92$ & $<.1$ & $<.1$ & $<.1$ & $<10$ & $<1.0$ & $<1.0$ & $<.1$ & $<1$ \\
\hline \multicolumn{9}{|c|}{ Pantano Wash at Broadway Boulevard } \\
\hline $11-02-87$ & $<.1$ & $<.1$ & $<.1$ & $<10$ & $<1.0$ & $<1.0$ & $<.1$ & $<.1$ \\
\hline $02-20-92$ & $<.1$ & $<.1$ & $<.1$ & $<10$ & $<1.0$ & $<1.0$ & .5 & $<.1$ \\
\hline \multicolumn{9}{|c|}{ Alamo Wash at Glenn Street } \\
\hline $07-28-87$ & .9 & .12 & .2 & $<10$ & 13 & $<1.0$ & $<.1$ & $<1$ \\
\hline $02-10-92$ & .1 & $<1$ & $<.1$ & $<10$ & $<1.0$ & $<1.0$ & $<.1$ & $<.1$ \\
\hline \multicolumn{9}{|c|}{ Rilito Creek at Dodge Boulevard } \\
\hline $08-03-88$ & $<.1$ & $<.1$ & $<.1$ & $<10$ & $<1.0$ & $<1.0$ & $<.1$ & $<.1$ \\
\hline $02-20-92$ & $<.1$ & $<.1$ & $<.1$ & $<10$ & $<1.0$ & $<1.0$ & $<.1$ & $<.1$ \\
\hline
\end{tabular}


Table 11. Analytical results of bottom-sediment samples, Rillito Creek basin-Continued Priority pollutants

\begin{tabular}{|c|c|c|c|c|c|c|c|c|c|}
\hline Date & $\begin{array}{l}\text { Para- } \\
\text { chloro- } \\
\text { meta } \\
\text { cresol } \\
(\mu \mathrm{g} / \mathrm{kg})\end{array}$ & $\begin{array}{c}\text { 2-Chloro- } \\
\text { phenol } \\
\text { ( } \mu \mathrm{g} / \mathrm{kg})\end{array}$ & $\begin{array}{l}\text { 2,4-DI- } \\
\text { chloro- } \\
\text { phenol } \\
(\mu \mathrm{g} / \mathrm{kg})\end{array}$ & $\begin{array}{c}2,4 \\
\text { Dichloro- } \\
\text { phenol, } \\
(\mu \mathrm{g} / \mathrm{kg})\end{array}$ & $\begin{array}{l}4,6-D i- \\
\text { nitro- } \\
\text { ortho } \\
\text { cresol } \\
(\mu g / \mathrm{kg})\end{array}$ & $\begin{array}{c}2,4 \\
\text { Dinitro- } \\
\text { phenol } \\
(\mu \mathrm{g} / \mathrm{kg})\end{array}$ & $\begin{array}{l}\text { 2-Nitro- } \\
\text { phenol } \\
(\mu g / k g)\end{array}$ & $\begin{array}{l}\text { 4-Nitro- } \\
\text { phenol } \\
(\mu g / k g)\end{array}$ & $\begin{array}{l}\text { Penta- } \\
\text { chloro- } \\
\text { phenol } \\
(\mu \mathrm{g} / \mathrm{kg})\end{array}$ \\
\hline \multicolumn{10}{|c|}{ Tanque Verde Creek at Tucson } \\
\hline $08-22-88$ & $<600$ & $<200$ & $<200$ & $<200$ & $<600$ & $<600$ & $<200$ & $<600$ & $<600$ \\
\hline $02-21-92$ & $<600$ & $<200$ & $<200$ & $<200$ & $<600$ & $<600$ & $<200$ & $<600$ & $<600$ \\
\hline \multicolumn{10}{|c|}{ Pentino Whsh of Brondway Boulevard } \\
\hline $11-02-87$ & $<600$ & $<200$ & $<00$ & $<200$ & $<600$ & $<600$ & $<200$ & $<600$ & $<600$ \\
\hline $02-20-92$ & $<600$ & $<200$ & $<200$ & $<200$ & $<600$ & $<600$ & $<200$ & $<600$ & $<600$ \\
\hline \multicolumn{10}{|c|}{ Alamo Wash at Glenistred } \\
\hline $07-28-87$ & $<600$ & $<200$ & $<200$ & $<00$ & $<600$ & $<600$ & $<00$ & $<600$ & $<600$ \\
\hline $02-10-92$ & $<600$ & $<200$ & $<200$ & $<200$ & $<600$ & $<600$ & $<200$ & $<600$ & $<600$ \\
\hline \multicolumn{10}{|c|}{ Rilito Creek at Dod o Bouleverd } \\
\hline $08-03-88$ & $<600$ & $<200$ & $<200$ & 200 & $<600$ & $<600$ & $<200$ & $<600$ & $<600$ \\
\hline $02-20-92$ & $<600$ & $<200$ & $<200$ & $<200$ & $<600$ & $<600$ & $<200$ & $<600$ & $<600$ \\
\hline
\end{tabular}

\begin{tabular}{|c|c|c|c|c|c|c|c|c|c|}
\hline Date & $\begin{array}{c}\text { Phenol } \\
\text { (C6h-50h) } \\
(\mu \mathrm{g} / \mathrm{kg})\end{array}$ & $\begin{array}{l}\text { 2,4,6-Trl- } \\
\text { chloro- } \\
\text { phenol } \\
(\mu \mathrm{g} / \mathrm{kg})\end{array}$ & $\begin{array}{c}\text { Ace- } \\
\text { naph- } \\
\text { thene } \\
(\mu \mathrm{g} / \mathrm{kg})\end{array}$ & $\begin{array}{c}\text { Ace- } \\
\text { naph- } \\
\text { thylene } \\
(\mu \mathrm{g} / \mathrm{kg})\end{array}$ & $\begin{array}{c}\text { Anthra- } \\
\text { cene } \\
(\mu g / k g)\end{array}$ & $\begin{array}{c}\text { Benzo A } \\
\text { anthra- } \\
\text { cene 1,2- } \\
\text { benzan- } \\
\text { thranene } \\
(\mu \mathrm{g} / \mathrm{kg})\end{array}$ & $\begin{array}{c}\text { Benzo B } \\
\text { fluor- } \\
\text { anthene } \\
(\mu \mathrm{g} / \mathrm{kg})\end{array}$ & $\begin{array}{c}\text { Benzo K } \\
\text { fluor- } \\
\text { anthene } \\
(\mu \mathrm{g} / \mathbf{k g})\end{array}$ & $\begin{array}{c}\text { Benzo A } \\
\text { pyrene } \\
(\mu \mathrm{g} / \mathbf{k g})\end{array}$ \\
\hline \multicolumn{10}{|c|}{ Tanque Verde Creek at Tueson } \\
\hline $08-22-88$ & $<200$ & $<600$ & $<200$ & $<200$ & $<200$ & $<400$ & $<400$ & $<400$ & $<400$ \\
\hline $02-21-92$ & $<200$ & $<600$ & $<200$ & $<200$ & $<200$ & $<400$ & $<400$ & $<400$ & $<400$ \\
\hline \multicolumn{10}{|c|}{ Pantino Wash at Broadway Boulevard } \\
\hline $11-02-87$ & $<200$ & $<600$ & $<200$ & $<200$ & $<00$ & $<400$ & $<400$ & $<400$ & $<400$ \\
\hline $02-20-92$ & $<200$ & $<600$ & $<200$ & $<200$ & $<200$ & $<400$ & $<400$ & $<400$ & $<400$ \\
\hline \multicolumn{10}{|c|}{ Alamo Wosh at Glenn Street } \\
\hline $07-28-87$ & $<200$ & $<600$ & $<200$ & $<200$ & $<200$ & 710 & 1,100 & 930 & 850 \\
\hline $02-10-92$ & $<200$ & $<600$ & $<200$ & $<200$ & $<200$ & $<400$ & $<400$ & $<400$ & $<400$ \\
\hline \multicolumn{10}{|c|}{ Rillito Creek at Dodge Boulevard } \\
\hline 08-03-88 & $<00$ & $<600$ & $<200$ & 200 & $<200$ & $<400$ & $<400$ & $<400$ & $<400$ \\
\hline $02-20-92$ & $<200$ & $<600$ & $<200$ & $<200$ & $<200$ & $<400$ & $<400$ & $<400$ & $<400$ \\
\hline Date & $\begin{array}{c}\text { Benzogh I } \\
\text { perylene 1, } \\
\text { 12-benzo- } \\
\text { perylene } \\
(\mu \mathrm{g} / \mathrm{kg})\end{array}$ & $\begin{array}{c}\text { DI-N- } \\
\text { butyl } \\
\text { phthan- } \\
\text { ate } \\
(\mu \mathrm{g} / \mathrm{kg})\end{array}$ & $\begin{array}{c}\text { Bis (2- } \\
\text { chloro- } \\
\text { ethoxy) } \\
\text { methene } \\
(\mu \mathrm{g} / \mathrm{kg})\end{array}$ & $\begin{array}{l}\text { Bls (2- } \\
\text { chloro- } \\
\text { ethyl) } \\
\text { ether } \\
(\mu \mathrm{g} / \mathrm{kg})\end{array}$ & $\begin{array}{c}4 \\
\text { Bromo- } \\
\text { phenyl } \\
\text { phenyl } \\
\text { ether } \\
(\mu \mathrm{g} / \mathrm{kg})\end{array}$ & $\begin{array}{c}\text { 2-Chloro- } \\
\text { naphtha- } \\
\text { lene } \\
(\mu \mathrm{g} / \mathrm{kg})\end{array}$ & $\begin{array}{l}\text { 4-Chloro- } \\
\text { phenyl } \\
\text { phenyl } \\
\text { ether } \\
(\mu \mathrm{g} / \mathrm{kg})\end{array}$ & $\begin{array}{c}\text { Chrysene } \\
(\mu g / k g)\end{array}$ & $\begin{array}{c}\text { 1,2,4-Trl- } \\
\text { chloro- } \\
\text { benzene } \\
(\mu \mathrm{g} / \mathrm{kg}\end{array}$ \\
\hline \multicolumn{10}{|c|}{ Tanque Verde Creek at Ticson } \\
\hline $08-22-88$ & $<400$ & $<200$ & $<200$ & $<200$ & $<200$ & $<00$ & $\ldots$ & $<400$ & $<200$ \\
\hline $02-21-92$ & $<400$ & $<200$ & $<200$ & $<200$ & $<200$ & $<200$ & $\cdots$ & $<400$ & $<200$ \\
\hline \multicolumn{10}{|c|}{ Pantano Wash at Brosdway Boulevard } \\
\hline $11-02-87$ & $<400$ & $<200$ & $<200$ & $<200$ & $<200$ & $<200$ & $\cdots$ & $<400$ & $<200$ \\
\hline $02-20-92$ & $<400$ & $<200$ & $<200$ & $<200$ & $<200$ & $<200$ & $\cdots$ & $<400$ & $<200$ \\
\hline \multicolumn{10}{|c|}{ Alamo Wash ul Clenn Street } \\
\hline $07-28-87$ & 760 & $<200$ & $<200$ & $<00$ & $<200$ & $<200$ & $<00$ & 1,200 & $<200$ \\
\hline $02-10-92$ & $<400$ & $<200$ & $<200$ & $<200$ & $<200$ & $<200$ & $<200$ & $<400$ & $<200$ \\
\hline \multicolumn{10}{|c|}{ Rillito Creek at Dodge Boulevard } \\
\hline $08-03-88$ & $<400$ & $<200$ & $<200$ & $<200$ & $<200$ & $<200$ & -- & $<400$ & $<200$ \\
\hline $02-20-92$ & $<400$ & $<200$ & $<200$ & $<200$ & $<200$ & $<200$ & -- & $<400$ & $<200$ \\
\hline
\end{tabular}


Table 11. Analytical results of bottom-sediment samples, Rillito Creek basin-Continued

Priority pollutants-Continued

\begin{tabular}{|c|c|c|c|c|c|c|c|c|c|}
\hline Date & $\begin{array}{c}1,2,5,6- \\
\text { Dibenzan- } \\
\text { thracene } \\
(\mu \mathrm{g} / \mathrm{kg})\end{array}$ & $\begin{array}{c}\text { Di-N-butyl- } \\
\text { phthalate } \\
(\mu g / \mathrm{kg})\end{array}$ & $\begin{array}{c}\text { 1,2-Dl- } \\
\text { chloro- } \\
\text { benzene } \\
(\mu \mathrm{g} / \mathrm{kg})\end{array}$ & $\begin{array}{c}\text { 1,3-Di- } \\
\text { chloro- } \\
\text { benzene } \\
(\mu \mathrm{g} / \mathrm{kg})\end{array}$ & $\begin{array}{c}\text { 1,4-Di- } \\
\text { chloro- } \\
\text { benzene } \\
(\mu \mathrm{g} / \mathrm{kg})\end{array}$ & $\begin{array}{c}\text { Diethyl- } \\
\text { phthalate } \\
(\mu \mathrm{g} / \mathrm{kg})\end{array}$ & $\begin{array}{c}\text { Di- } \\
\text { methyl- } \\
\text { phthalate } \\
(\mu \mathrm{g} / \mathrm{kg})\end{array}$ & $\begin{array}{c}\text { 2,4-Di- } \\
\text { nitro- } \\
\text { toluene } \\
\text { ( } \mu \mathrm{g} / \mathrm{kg} \text { ) }\end{array}$ & $\begin{array}{c}\text { 2,6-DI- } \\
\text { nitro- } \\
\text { toluene } \\
(\mu \mathrm{g} / \mathrm{kg})\end{array}$ \\
\hline \multicolumn{10}{|c|}{ Tanque Verde Creek at Tucson } \\
\hline $08-22-88$ & $<400$ & 200 & $<200$ & 200 & $<200$ & $<200$ & $<00$ & $<200$ & $<200$ \\
\hline $02-21-92$ & $<400$ & $<200$ & $<200$ & $<200$ & $<200$ & $<200$ & $<200$ & $<200$ & $<200$ \\
\hline \multicolumn{10}{|c|}{ Pantano Wash a Broadway Bouleverd } \\
\hline $11-02-87$ & $<400$ & $<200$ & $<200$ & $<200$ & $<200$ & $<200$ & $<200$ & $<200$ & $<200$ \\
\hline $02-20-92$ & $<400$ & $<200$ & $<200$ & $<200$ & $<200$ & $<200$ & $<200$ & $<200$ & $<200$ \\
\hline \multicolumn{10}{|c|}{ Alamo Wash ot Glenn Street } \\
\hline $07-28-87$ & $<400$ & $<200$ & $<200$ & $<200$ & $<200$ & $<200$ & $<200$ & $<200$ & $<200$ \\
\hline $02-10-92$ & $<400$ & $<00$ & $<200$ & $<200$ & $<200$ & $<200$ & $<200$ & $<200$ & $<200$ \\
\hline \multicolumn{10}{|c|}{ Rillito Creek af Dodge Boulevard } \\
\hline $08-03-88$ & $<400$ & $<200$ & $<200$ & 200 & $<200$ & $<200$ & $<200$ & $<200$ & $<200$ \\
\hline $02-20-92$ & $<400$ & $<00$ & $<200$ & $<200$ & $<200$ & $<200$ & $<200$ & $<200$ & $<00$ \\
\hline
\end{tabular}

\begin{tabular}{|c|c|c|c|c|c|c|c|c|c|}
\hline Date & $\begin{array}{c}\text { Di-n- } \\
\text { octyl } \\
\text { phthalate } \\
(\mu \mathrm{g} / \mathrm{kg})\end{array}$ & $\begin{array}{c}\text { Bis (2- } \\
\text { ethyl- } \\
\text { hexly) } \\
\text { phthalate } \\
(\mu \mathrm{g} / \mathrm{kg})\end{array}$ & $\begin{array}{c}\text { Fluorene } \\
(\mu \mathbf{g} / \mathbf{k g})\end{array}$ & $\begin{array}{c}\text { Fluoran- } \\
\text { thene } \\
(\mu \mathrm{g} / \mathrm{kg})\end{array}$ & $\begin{array}{c}\text { Hexa- } \\
\text { chloro- } \\
\text { benzene } \\
(\mu \mathrm{g} / \mathrm{kg})\end{array}$ & $\begin{array}{l}\text { Hexa- } \\
\text { chloro- } \\
\text { but- } \\
\text { adlence } \\
(\mu \mathrm{g} / \mathrm{kg})\end{array}$ & $\begin{array}{l}\text { Hexa- } \\
\text { cholor- } \\
\text { cyclo- } \\
\text { pent- } \\
\text { adlene } \\
(\mu g / k g)\end{array}$ & $\begin{array}{l}\text { Hexa- } \\
\text { chloro- } \\
\text { ethane } \\
(\mu \mathrm{g} / \mathrm{kg})\end{array}$ & $\begin{array}{c}\text { Indeno- } \\
(1,2,3-c d) \\
\text { pyrene } \\
(\mu \mathrm{g} / \mathrm{kg})\end{array}$ \\
\hline \multicolumn{10}{|c|}{ Tanque Verde Creek at Tucson } \\
\hline $08-22-88$ & $<400$ & 200 & 200 & 390 & 200 & $<200$ & $<200$ & $<200$ & $<200$ \\
\hline $02-21-92$ & $<400$ & $<200$ & $<200$ & $<200$ & $<200$ & $<200$ & $<200$ & $<200$ & $<200$ \\
\hline \multicolumn{10}{|c|}{ Pantano Wasth at Broadvi ay Boulevard } \\
\hline $11-02-87$ & $<400$ & $<200$ & $<200$ & $<200$ & $<200$ & $<200$ & $<200$ & $<200$ & $<200$ \\
\hline $02-20-92$ & $<400$ & $<200$ & $<200$ & $<200$ & $<00$ & $<200$ & $<200$ & $<200$ & $<200$ \\
\hline \multicolumn{10}{|c|}{ Ala mo Whsh at Glenn Street } \\
\hline $07-28-87$ & $<400$ & 1,800 & $<200$ & 2,300 & $<200$ & $<200$ & $<200$ & $<200$ & 830 \\
\hline $02-10-92$ & $<400$ & $<200$ & $<200$ & 220 & $<200$ & $<200$ & $<200$ & $<200$ & 430 \\
\hline \multicolumn{10}{|c|}{ Rlilito Creek if Dodge Boulevard } \\
\hline $08-03-88$ & $<400$ & $<200$ & $<200$ & $<200$ & 200 & $<00$ & $<200$ & 200 & $<200$ \\
\hline $02-20-92$ & $<400$ & $<200$ & $<200$ & $<200$ & $<200$ & $<200$ & $<200$ & $<200$ & $<400$ \\
\hline Date & $\begin{array}{l}\text { Iso- } \\
\text { Phorone } \\
(\mu \mathrm{g} / \mathrm{kg})\end{array}$ & $\begin{array}{l}\text { Naph- } \\
\text { thalene } \\
\text { ( } \mu \mathrm{gg} / \mathrm{kg})\end{array}$ & $\begin{array}{c}\text { Nitro- } \\
\text { benzene } \\
(\mu \mathrm{g} / \mathbf{k g})\end{array}$ & & $\begin{array}{l}\text { itro- } \\
\text { dl- } \\
\text { chyl- } \\
\text { Ine } \\
\text { (kg) }\end{array}$ & $\begin{array}{l}\text { N-nltro- } \\
\text { sodi- } \\
\text { phenyl- } \\
\text { amine } \\
(\mu g / k g)\end{array}$ & $\begin{array}{l}\text { Nitro- } \\
\text { sodl-n- } \\
\text { propyl- } \\
\text { amine } \\
(\mu \mathrm{g} / \mathrm{kg})\end{array}$ & $\begin{array}{l}\text { Phen- } \\
\text { anthrene } \\
(\mu \mathrm{g} / \mathrm{kg})\end{array}$ & $\begin{array}{l}\text { Pyrene } \\
(\mu g / k g)\end{array}$ \\
\hline \multicolumn{10}{|c|}{ Tanque Verde Creek at Tucson } \\
\hline $08-22-88$ & $<200$ & $<200$ & $<00$ & & 200 & $<200$ & $<200$ & $<200$ & 480 \\
\hline $02-21-92$ & $<200$ & $<200$ & $<200$ & & 200 & $<200$ & $<200$ & $<200$ & 480 \\
\hline \multicolumn{10}{|c|}{ Pantano Wash at Broadway Boulevard } \\
\hline $11-02-87$ & $<200$ & $<200$ & $<200$ & & 200 & $<200$ & $<200$ & 200 & $<200$ \\
\hline $02-20-92$ & $<200$ & $<200$ & $<00$ & & 200 & $<200$ & $<200$ & $<200$ & $<00$ \\
\hline \multicolumn{10}{|c|}{ Alemo Wash at Gleon Streot } \\
\hline $07-28-87$ & $<200$ & $<200$ & $<200$ & & 200 & $<200$ & $<200$ & 620 & 2,000 \\
\hline $02-10-92$ & $<200$ & $<200$ & $<200$ & & 200 & $<200$ & $<200$ & 620 & 2,000 \\
\hline \multicolumn{10}{|c|}{ Rilito Creek at Dodge Boulevard } \\
\hline $08-03-88$ & $<200$ & $<200$ & $<200$ & & 200 & $<200$ & $<200$ & $<200$ & $<200$ \\
\hline $02-20-92$ & $<200$ & $<200$ & $<200$ & & 200 & $<200$ & $<200$ & $<200$ & $<200$ \\
\hline
\end{tabular}


Table 11. Analytical results of bottom-sediment samples, Rillito Creek basin-Continued Inorganic carbon plus organic carbon, inorganic carbon, and oil and grease

\begin{tabular}{|c|c|c|c|}
\hline Date & $\begin{array}{l}\text { Carbon organic plus organic, total } \\
\qquad(g / k g \text { as } C)\end{array}$ & $\begin{array}{l}\text { Carbon, Inorganic total } \\
\text { (g/kg as C) }\end{array}$ & $\begin{array}{l}\text { Oll and grease, total } \\
\text { gravimetrlc (mg/kg) }\end{array}$ \\
\hline \multicolumn{4}{|c|}{ Tanque Verde Creek at Tucson } \\
\hline $08-22-88$ & 8.2 & 2.1 & $<1,000$ \\
\hline $07-25-89$ & 13 & 1.3 & -- \\
\hline $02-21-92$ & 1 & .1 & $<1,000$ \\
\hline \multicolumn{4}{|c|}{ Pantano Wash af Broudway Boulevard } \\
\hline $11-02-87$ & 3.6 & 1.7 & $<1,000$ \\
\hline $02-20-92$ & 3.3 & 2.2 & $<1,000$ \\
\hline \multicolumn{4}{|c|}{ Alamo Wash at Glenn Street } \\
\hline $07-28-87$ & 9.0 & 7.3 & $<1,000$ \\
\hline $02-10-92$ & 2.2 & 1.7 & $<1,000$ \\
\hline \multicolumn{4}{|c|}{ Rillito Creek at Dodge Boulevard } \\
\hline $08-03-88$ & 3.1 & 2.0 & $<1,000$ \\
\hline $02-20-92$ & 1.4 & .8 & $<1,000$ \\
\hline
\end{tabular}

